Omega-P, Inc.

291 Whitney Avenue - Suite 401

New Haven, CT 06511

\title{
FERROELECTRIC SWITCH FOR A HIGH-POWER Ka-BAND ACTIVE PULSE COMPRESSOR
}

\author{
J. L. Hirshfield, Principal Investigator \\ tel: (203) 789-1164, e-mail: jay@ omega-p.com
}

\section{Final Report on Phase II DoE SBIR grant DE-FG02-05 ER84367}

\begin{abstract}
Results are presented for design of a high-power microwave switch for operation at 34.3 GHz, intended for use in an active RF pulse compressor. The active element in the switch is a ring of ferroelectric material, whose dielectric constant can be rapidly changed by application of a high-voltage pulse. As envisioned, two of these switches would be built into a pair of delay lines, as in SLED-II at SLAC, so as to allow 30-MW $\mu$ s-length Ka-band pulses to be compressed in time by a factor-of- 9 and multiplied in amplitude to generate $200 \mathrm{MW}$ peak power pulses. Such high-power pulses could be used for testing and evaluation of high-gradient mm-wave accelerator structures, for example. Evaluation of the switch design was carried out with an X-band $(11.43 \mathrm{GHz})$ prototype, built to incorporate all the features required for the Kaband version.
\end{abstract}

Key words: RF pulse compressor, ferroelectric switches, active pulse compression

Work on this project was carried out by S.V. Shchelkunov, S.Yu. Kaazakov,* V.P. Yakovlev,* O.A. Nezhevenko,* and J.L. Hirshfield of Omega-P, Inc.; A.D. Kanareykin and E. Nenasheva of Euclid TechLabs, LLC, and S.H. Gold of US Naval Research Laboratory. *Currently employed at Fermi National Accelerator Laboratory.

December 18, 2013 


\section{TABLE OF CONTENTS}

I. INTRODUCTION 3

II. TECHNICAL APPROACH 4

III. TECHNICAL FEASIBILITY 7

IIIa. Technical objectives $\quad 7$

IIIlb. General scheme 7

IIIc, Optimization of coupling 8

IIId. Ferroelectric switch 10

IIIe. Ferroelectric materials 16

IIIf. Tolerance analysis 19

IIIg. Engineering design concept for the high-power switch 20

IIIh. Experimental test setup 20

$\begin{array}{ll}\text { REFERENCES } & 23\end{array}$

APPENDIX I - Theoretical analysis $\quad 25$

APPENDIX II - Copies of relevant published papers $\quad$ 26ff

APPENDIX III - Copies of relevant technical presentations $\quad 26 f f$ 


\section{INTRODUCTION}

Under Topic 37b in the DoE 2005 SBIR Program Solicitation, proposals were sought "...for passive and active RF components such as over-moded mode converters from rectangular to circular waveguide and vice versa, high-power RF windows, circulators, isolators, switches, and quasi-optical components...." Omega-P, Inc. submitted a proposal in response, entitled "Ferroelectric Switch for a High-Power Ka-Band Active Pulse Compressor," and was awarded Phase I and II grants DE-FG02-05 ER 84367, with the same title. The overall goal of this R\&D project was to develop a Ka-band switch at $34.3 \mathrm{GHz}$ for an active pulse compressor with a peak power output of about $200 \mathrm{MW}$. A Ka-band RF source with this peak power could be used in basic studies of RF breakdown and in development of structures that can sustain high acceleration gradients for use in a future multi-TeV collider, such as CLIC [1].

The need for experimental facilities for these basic investigations and for development of new high-gradient structures had intensified, as the accelerator community came to accept the need for fundamental studies of processes that limit RF fields in room temperature ("warm") structures. As far back as March 3, 2006 Dr. Robin Staffin, then Associate Director, Office of High Energy Physics DoE, testified to HEPAP regarding the FY2007 budget on the request for

"... significant increase....in the long-range R\&D program that supports fundamental research into the physics of....'accelerator science.' The goal is to enable the restoration of the accelerator science research program to the level needed to support long-term R\&D on new particle acceleration techniques and technologies, such as novel particle acceleration concepts, very high gradient accelerating structures, and user facilities to test these concepts...."

The project reported on here by Omega-P is for a key technological component needed for production of the high power RF pulses required to carry out experiments referred to by Dr. Staffin. This key component is an efficient high-power RF switch to allow rapid change in the coupling between a delay line where RF energy from a pulsed source is stored, so as to release the energy as a shorter, higher intensity pulse to be delivered to a structure under test. With such a high-power switch it was anticipated that 30-MW, 0.5-0.7 $\mu$ s RF pulses from the Yale/OmegaP 34.3-GHz magnicon could be compressed to become 200-MW, 50-70 ns pulses for exposing test structures to RF surface electric fields of many 100's of MV/m.

Development of this active pulse compressor was initially encouraged by test results of the $34.3 \mathrm{GHz}$ third-harmonic magnicon amplifier [2] developed by Omega-P; it had a design power of over $40 \mathrm{MW}$ in $1 \mu \mathrm{sec}$ pulses. The fully assembled tube is shown in Fig. 1. RF conditioning allowed the magnicon to produce an output power of up to $20 \mathrm{MW}$ in $0.5 \mu$ s pulses, with a gain of $54 \mathrm{~dB}$. Fig. 2 shows a sample output waveform. Slotted line measurements confirm that the output is monochromatic to within a margin of at least $30 \mathrm{~dB}$. These preliminary results constituted record values for a millimeter-wave accelerator-class amplifier. However, since those encouraging early results, the Omega-P Ka-band magnicon has failed to reach its design output level of $40 \mathrm{MW}$. As a result, it has been decommissioned, the cathode in its electron gun has been replaced, and fabrication is awaited of a newly-designed RF cavity chain that embodies a novel two-chamber output cavity. As shall be seen, the decommissioning of the Ka-band magnicon led to diversion of this project from Ka- to X-band, where an alternative test facility is available. 


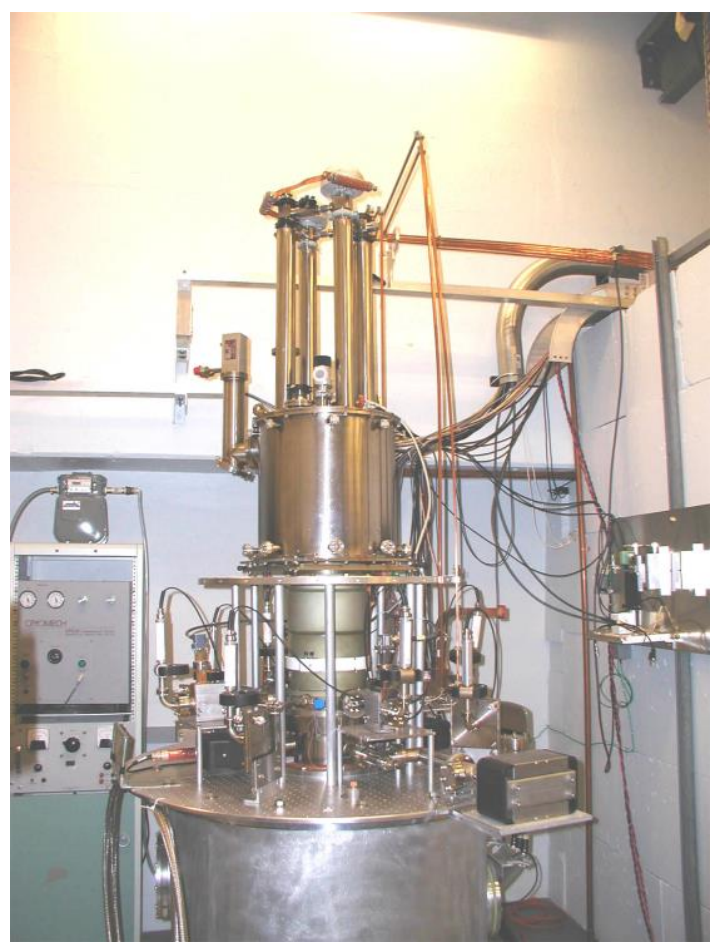

Figure 1. A general view of the $34.3 \mathrm{GHz}$ magnicon.

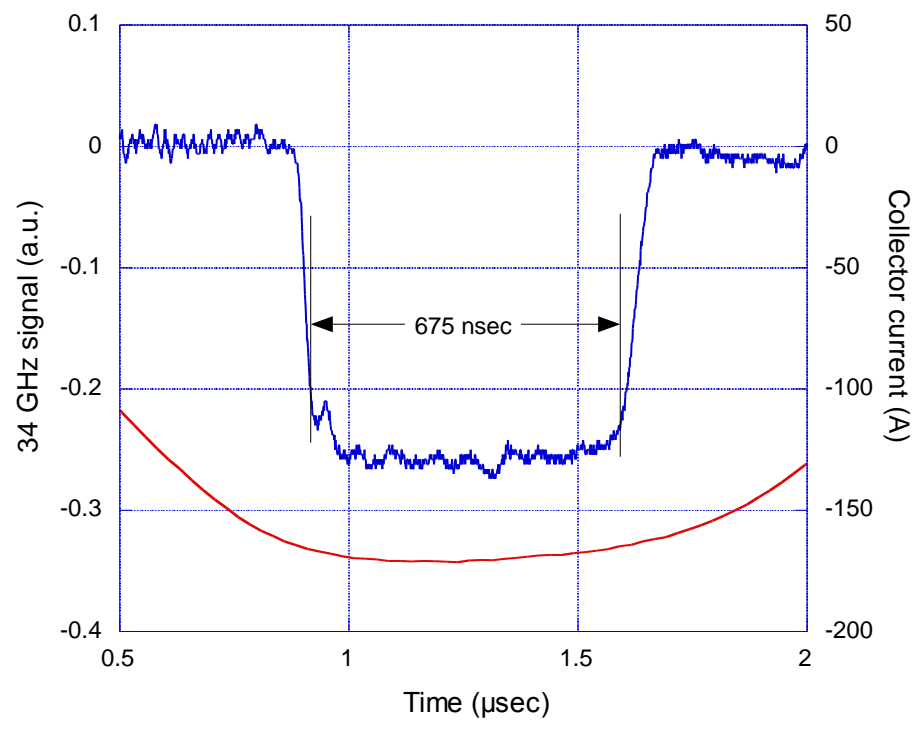

Figure 2. Sample RF output waveform from the 34-GHz magnicon (blue) and magnicon beam current (red).

\section{TECHNICAL APPROACH}

The Omega-P project described here had as its goal the development of a $34.3 \mathrm{GHz}, 200$ MW, active pulse compressor fed by the Yale/Omega-P magnicon, with a view towards later possibly transitioning the technology to $30 \mathrm{GHz}$, once tests in Phase II confirm the expected performance of the pulse compressor. This transition would have made the technology available for studies of structures for use in CLIC - although scaled structures could be tested in the meantime at Yale at $34.3 \mathrm{GHz}$. In the meantime, the CLIC RF technology had switched from 30 $\mathrm{GHz}$ to $12 \mathrm{GHz}$; this removed urgency from the Omega-P Ka-band project, and enforced the decision for Omega-P to focus attention from Ka- to X-band.

An example of a pulse compressor based on a SLAC design known as SLED-II is shown in Fig. 3 [4]. The system includes storage cavities or, in this particular case, delay lines that allow formation of a rectangular pulse; coupling irises; and a 3-dB coupler. It operates the following way: initially the energy from the long klystron pulse is stored in the cavity or delay line, and after the klystron phase changes by $180^{\circ}$ the energy is expelled into the load (accelerating structure) in a short pulse with a higher power.

The SLED-II system suffers from two types of losses that reduce its intrinsic efficiency: (i) during the charging phase some of the energy is reflected and never gets inside the line, and (ii) after the phase is reversed the energy inside the line is not discharged completely during the compressed pulse time period. These two effects make the intrinsic efficiency of SLED-II deteriorate very rapidly as the compression ratio increases (see Table I [5]), and in practice make it impractical to achieve the desired compression ratio of 8-10 to 1. 


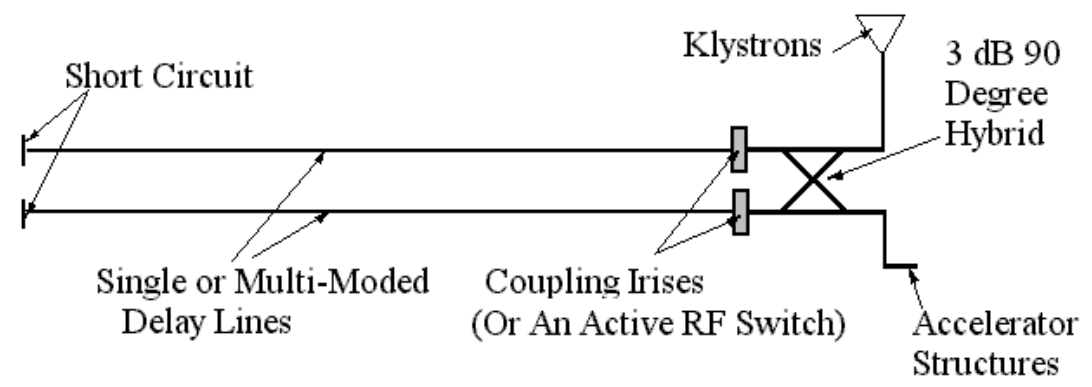

Fig. 3. Resonant Delay Line Pulse Compression System (SLED-II).

Table I. Ideal power gain $G_{\text {ideal }}$ (assuming no Ohmic losses), and intrinsic efficiency $\eta_{\text {int }}$ for different compression ratios [5]. $C_{r}$ is the compression ratio (or number of bins).

\begin{tabular}{|c|c|c|c|c|}
\hline & \multicolumn{2}{|c|}{ SLED II } & \multicolumn{2}{|c|}{$\begin{array}{l}\text { active pulse } \\
\text { compressor, } \\
\text { switching after } \\
\text { the last bin }\end{array}$} \\
\hline$C_{\mathrm{r}}$ & $G_{\text {ideal }}$ & $\eta_{\text {int }}$ & $G_{\text {ideal }}$ & $\eta_{\text {int }}$ \\
\hline 4 & 3.440 & 0.860 & 3.285 & 0.821 \\
\hline 5 & 4.022 & 0.804 & 4.094 & 0.819 \\
\hline 6 & 4.477 & 0.746 & 4.905 & 0.818 \\
\hline 7 & 4.844 & 0.692 & 5.717 & 0.817 \\
\hline 8 & 5.148 & 0.644 & 6.530 & 0.816 \\
\hline 9 & 5.405 & 0.601 & 7.343 & 0.816 \\
\hline 10 & 5.625 & 0.562 & 8.156 & 0.816 \\
\hline 11 & 5.816 & 0.529 & 8.97 & 0.815 \\
\hline 12 & 5.984 & 0.499 & 9.838 & 0.815 \\
\hline
\end{tabular}

One can see, for SLED II, that for a compression ratio $C_{r}=10$ the ideal power gain $G_{\text {ideal }}$ is only equal to 5.625. The actual power gain will be even lower due to attenuation in the delay lines, but not substantially because the delay lines can be built with a very high quality factor [6]. But achievement of an efficiency $>50 \%$ would appear unlikely.

In the active pulse compressor the coupling irises (see Fig. 2) are replaced with fast highpower RF switches, which change the delay line coupling during the RF pulse. In the simplest case, the switching takes place once right after the process of energy storage is completed, i.e., right after the klystron pulse ends (see e.g. [5]); or in another words, after the last bin. This simplest active pulse compressor scheme already allows substantial increase of efficiency. From Table I one can see that the efficiency is $\eta_{\text {int }}=0.815$ compare to $\sim 0.5$ for SLED-II even for a high compression ratio $C_{r}=12$. There are two reasons why this high efficiency is achieved. First, little or no energy is left in the delay lines after the output pulse is finished and second, optimization of coupling for the charging phase allows a reduction in energy reflection compared to SLED-II. 
In an attempt to circumvent limitations of passive pulse compressors, various concepts of high power switches for active RF pulse compressors have received attention. These involve switches with optically-varied silicon mirrors [7], PIN/NIP diode arrays [8], and plasmas [9]. Until recently, none of the tested versions of these active pulse compressors achieved high enough power levels even at $11.4 \mathrm{GHz}$ : the maximum output power achieved is $10 \mathrm{MW}$ in the switch based on a PIN/NIP diode array active window [7], and $53 \mathrm{MW}$ in a compressor based on plasma switches [9].

For the present project, a Ka-band active pulse compressor with a switch based on use of electrically-controlled ferroelectric elements [10,11] was proposed. Ferroelectric elements received attention and are already used in low- to moderate-power (up to $100 \mathrm{~kW}$ peak) military and communication devices as fast tunable components [12,13] because they have the ability to operate at frequencies above $30 \mathrm{GHz}$ with reasonably low losses, and have very high tuning speed (intrinsic switching speed is reported to be $\sim 0.01 \mathrm{nsec}$ [13]). These characteristics together with the high electric breakdown strength and good vacuum properties make the ferroelectric switch an attractive candidate for use in high-power active RF pulse compressors. The rapid switching potential for ferroelectrics could perhaps make possible the imposition of several coupling changes during energy storage in a SLED-type pulse compressor, thereby allowing efficiency even higher than $80 \%$.

Ferroelectrics have an E-field-dependent dielectric permittivity $\varepsilon(\mathbf{E})$ that can be very rapidly altered by application of a bias voltage pulse. The switching time in most instances would be limited by the response time of the external electronic circuit that generates and transmits the high-voltage pulse, and can therefore be in the nsec range. Ferroelectric materials should have the following properties in order to be used in high-power RF switches for linear collider applications:

- the dielectric constant should not exceed 300-500 to avoid problems in the switch design caused by interference from high-order modes [10,11];

- the dielectric constant should change by 10 's of percent to provide the required switching properties;

- bias electric fields should be reasonable, namely not more than a few 10 's of $\mathrm{kV} / \mathrm{cm}$;

- the loss tangent should be about $\sim 2 \times 10^{-3}$ for K-band. At this loss tangent the losses in the ferroelectric material will not exceed a few percent.

Modern bulk ferroelectrics, such as barium strontium titanate $\left(\mathrm{Ba}_{\mathrm{x}} \mathrm{Sr}_{1-\mathrm{x}} \mathrm{TiO}_{3}\right.$, or BST) with $\varepsilon \sim 500$, have high enough electric breakdown strength $(100-200 \mathrm{kV} / \mathrm{cm})$ and do not require too high a bias electric field $(\sim 20-50 \mathrm{kV} / \mathrm{cm})$ to effect a significant change (20-30\%) in $\varepsilon$. Loss tangent for commercially-available samples of these materials is about $\sim 10^{-2}$ at $10 \mathrm{GHz}[12]$.

Euclid Concepts, LLC developed and tested for Omega-P a modified bulk ferroelectric based on a composition of BST ceramics, magnesium compounds, and rare-earth metal oxides that has a permittivity $\varepsilon=500$, and $20 \%$ change in permittivity for a bias electric field of 50 $\mathrm{kV} / \mathrm{cm}$. The loss tangent already achieved for the best samples is less than $4 \times 10^{-3}$ at $35 \mathrm{GHz}$ $[14,15,16]$. The availability of this ferroelectric already allows one to create a Ka-band highpower RF switch with a peak power of over $200 \mathrm{MW}$ and repetition rate up to $10 \mathrm{~Hz}$. 


\section{TECHNICAL FEASIBILITY}

\section{IIIa. Technical Objectives}

The technical objective of the project was first to develop a preliminary design of a highpower active pulse compressor based on use of resonance ferroelectric switches. The compressor design was to be tested by Omega-P and powered by the Omega-P $34.3 \mathrm{GHz}$ pulsed magnicon at an expected input power level of up to $30 \mathrm{MW}$ and pulse width of $\sim 1 \mu \mathrm{sec}$. Use of a pair of these switches with a pair of low-loss waveguide delay lines and a 3-dB hybrid would lead to production of a pulse of $70 \mathrm{nsec}$ width and a peak output power of about $200 \mathrm{MW}$. The design involved detailed analytical studies and numerical simulations of a switch cavity in order to minimize electric fields and power losses in the ferroelectric elements, providing at the same time required coupling during the stage of energy storage and the stage of energy extraction. A ferroelectric ring with required dimensions and characteristics was manufactured to demonstrate the required technical expertise.

\section{IIIb. General Scheme}

The schematic arrangement of an active pulse compressor can be explained using as an example the existing Omega-P two-channel Active Bragg Compressor (ABC-2) [17]. ABC-2 was installed in the X-band magnicon [18] test facility at Naval Research Laboratory, where it operated for testing switches employing plasma discharge tubes [9]. A diagram of ABC-2 is shown in Figure 4.

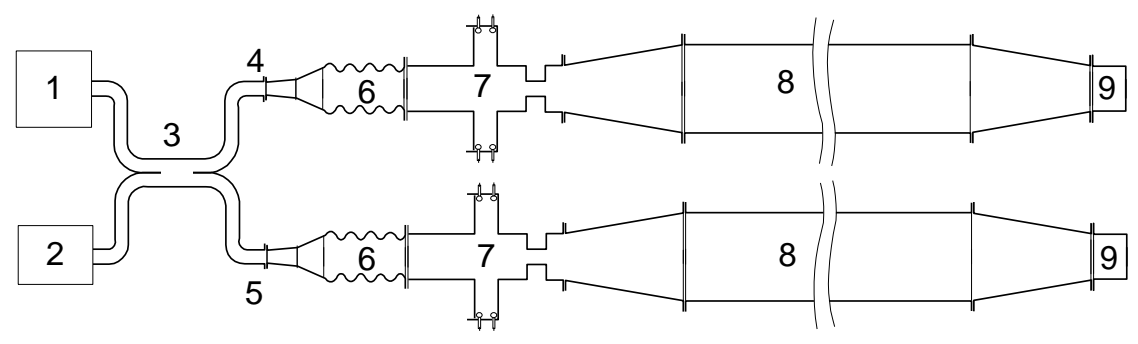

Figure. 4. Schematic diagram of the two-channel active pulse compressor. 1 - RF source, 2 - load (i.e., accelerator module), 3 -3-dB directional coupler, 4 - first channel of the pulse compressor, 5 - second channel of the pulse compressor, 6 - $\mathrm{TE}_{01}$-mode converter, 7 - electrically controlled switch, 8 - storage cavity, 9 - adjustable reflector.

In active pulsed compressors with resonance switches, the RF source supplies electromagnetic energy to fill a low-loss storage cavity coupled through an electricallycontrolled switch to a load (the accelerating structure). Compressor operation involves two steps: first, that of energy storage when the coupling to the storage cavity is small in order to provide good efficiency of filling; and second, that of energy extraction when the coupling is high to provide fast energy discharge into the accelerating structure. The coupling of the storage cavity with the RF source is controlled by changing the resonance frequency of the switch cavity. In general, it is possible to provide a fast change of resonance frequency of the cavity by rapidly changing the RF properties of an active element situated within the cavity. A schematic diagram of Omega-P's two-channel ABC-2 is shown in Fig. 2. As shown in Fig. 2, two cylindrical TE $01^{-}$ mode storage cavities (8) are each terminated with an adjustable short (9) at one end, and an 
electrically-controlled switch cavity (7) at the other end. Waveguides (4 and 5) are fed from the rf source (1) after the source power is split using a 3-dB hybrid coupler (3). Mode converters (6) transform the mode from $\mathrm{TE}_{10}$-rectangular to $\mathrm{TE}_{01}$-circular. Compressed output pulses are combined and absorbed in the load (2). This scheme is similar to SLED but for the addition of active elements (i.e., the resonance plasma switches). In the proposed Ka-band pulse compressor, the electrically-controlled switch employs ferroelectrics as the active elements instead of plasma discharge tubes.

Major issues that were addressed during the design phase were as follows:

1. Design of the pulse compressor which will allow the power in compressed pulse of $200 \mathrm{MW}$. Determination of the kind of the storage resonator in the form of a storage cavity or delay line and it's parameters was made.

2. Manufacturing was made of one ferroelectric ring having the design diameter of about 35 $\mathrm{mm}$, length of about $7 \mathrm{~mm}$, and thickness of $1-2 \mathrm{~mm}$. The material was to have required permittivity of 500 , and tunability of $20 \%$ at a bias field of $50 \mathrm{kV} / \mathrm{cm}$.

3. Investigation was made of the possibility of developing ferroelectric material having tunability $20 \%$ at $50 \mathrm{kV} / \mathrm{cm}$, but lower dielectric constant (up to 300). This will make easier the ring manufacturing and decrease losses in the ferroelectric.

4. Optimization of the switch cavity based on determined parameters of the storage resonator was made.

5. Preliminary engineering design considerations were made for the switch cavity. Tolerance analysis for the switch cavity was carried out, allowing determination of the necessary tuning range of the switch cavity.

Results of these efforts are described below.

\section{IIIc. Optimization of coupling}

The main design parameters of the proposed active pulse compressor with ferroelectric switches are given in Table II:

Table II. Parameters of the $34 \mathrm{GHz}$ active pulse compressor

\begin{tabular}{|c||c|}
\hline operating frequency, MHz & 34.272 \\
output power, MW & 200 \\
input power, MW & 30 \\
repetition rate, Hz & 10 \\
output pulse width, nsec & $50-70$ \\
input pulse width, nsec & $500-700$ \\
compression ratio & 10 \\
intrinsic efficiency, \% & 81.6 \\
efficiency of the delay line, \% & 89 \\
efficiency of the ferroelectric switch, \% & 92 \\
overall efficiency, \% & 67 \\
\hline
\end{tabular}

In the proposed pulse compressor, delay lines will be used because storage cavities do not allow flat output pulse necessary in many experiments. It is possible to use dual -mode SLAC-II 
type delay line [4] scaled for $34 \mathrm{GHz}$. For the output pulse width of $\sim 50 \mathrm{nsec}$ its length is about $4 \mathrm{~m}$. Another option is Kazakov's barrel delay line [19], which is even more compact. But the simplest way in an experimental setup is to use $7.5-\mathrm{m}$ long delay lines based on a circular waveguide operating in the $\mathrm{TE}_{01}$ mode. Note that a waveguide with low group velocity may be used, for example one with a length of 5-6 m, since the losses will not be greater.

Intrinsic efficiency $\eta$ of an active pulse compressor when the delay line discharging takes place after the pulse ends is determined by the formula [5]:

$$
\eta=\frac{(1+R)^{2}\left(1-R^{C}\right)^{2}}{\left(1-R^{2}\right) C}
$$

where $R$ is the switch reflection coefficient and $C$ is the compression ratio. When the losses in the switch are small, reflection R is related to the switch transmission $S$-parameter $\left|S_{12}\right|$ as

$$
R \approx\left(1-\left|S_{12}\right|^{2}\right)^{1 / 2}
$$

Intrinsic efficiency measures the fraction of incident energy from the RF source that is released after the input pulse ends; the balance is either reflected during charging of the delay line, or left in the delay line after the pulse ends. Calculated intrinsic efficiency versus the switch transmission coefficient during charging $\left|S_{12}\right|$ for $C=10$ is shown in Figure 5. The efficiency has a maximum value of 0.816 when $\left|S_{12}\right|=-6.5 \mathrm{~dB}$. Note that the efficiency is higher than $\sim 80 \%$ for a range of transmission values, namely $-7.6 \mathrm{~dB}<\left|S_{12}\right|<-5.6 \mathrm{~dB}$.

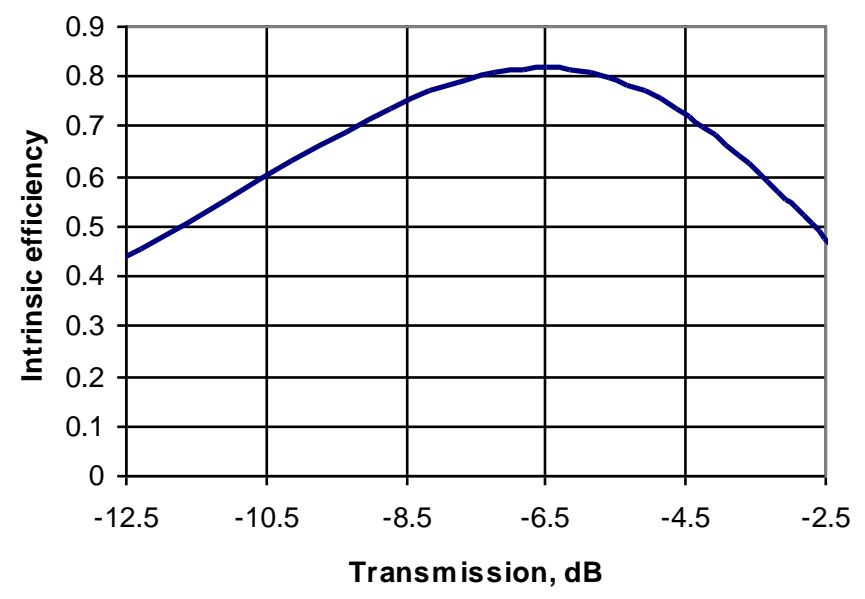

Figure 5. Intrinsic efficiency of the active pulse compressor $v s$. the switch transmission coefficient (in $\mathrm{dB}$ ). Delay line discharging takes place after the pulse ends.

For this example, the compression ratio $C$ is equal to 10 .

The required transmission coefficients of the switch cavity in both operating regimes are given in Table III. 
Table III. Switch cavity transmission in both operating regimes.

\begin{tabular}{|c|c|}
\hline regime & transmission, $\mathrm{dB}$ \\
\hline \hline energy storage & -6.5 \\
\hline energy extraction & $\sim 0$ \\
\hline
\end{tabular}

IIId. The ferroelectric switch

The ferroelectric switch was the scaled copy of the switch designed for X-band active pulse compressor [10,11]. The switch parameters are shown in Table IV, and the switch schematic is given in Fig. 6. The switch provides an optimal insulation in the energy storage regime. However, one can see from Table IV that RF field in the switch is high, it is about 40 $\mathrm{kV} / \mathrm{cm}$ in both energy storage and energy extraction regimes. This high RF field in the ferroelectrics can lead to undesirable nonlinear effects [20] that take place, for instance, in ferrite-tuned RF cavities for proton synchrotrons [21].

It is possible to show (see Appendix I) that the maximum electric field $E$ in the ferroelectric element of the resonant switch (the ring) is determined by the transmitted power $P_{0}$, the ring sizes (radius $r$, length $L$ and thickness $h$ ), and the change in dielectric constant $\Delta \varepsilon$ necessary to achieve the required change in transmission $T$. Thus

$$
E=\left(\frac{8 \alpha P_{0}}{\omega \Delta \varepsilon \cdot \pi r L h}\right)^{\frac{1}{2}}, \quad \text { where } \alpha=\sqrt{1-T^{2}} / T \text {. }
$$

For parameters given in Table III, the above equation gives a maximum electric field $E$ of 37 $\mathrm{kV} / \mathrm{cm}$ that practically coincides with the results of numerical calculations given in the same Table IV, namely $E=40 \mathrm{kV} / \mathrm{cm}$.

Table IV. Parameters of the initial design of Ka band ferroelectric switch

\begin{tabular}{|c|c|}
\hline operating frequency, $\mathrm{GHz}$ & 34.272 \\
\hline number of cavities & 2 \\
\hline cavity operating mode & $\mathrm{TE}_{031}$ \\
\hline cavity length, mm & 6.7 \\
\hline ferroelectric ring length, mm & 6.7 \\
\hline ferroelectric ring inner diameter, mm & 35.7 \\
\hline ferroelectric ring thickness, $\mathrm{mm}$ & 0.95 \\
\hline transmitted power, MW & 100 \\
\hline maximum electric field in ferroelectric for $\varepsilon=500, \mathrm{kV} / \mathrm{cm}$ & 40 \\
\hline power losses for $\varepsilon=500$ ( $\tan \delta=0.004), \mathrm{MW}$ & 6.7 \\
\hline transmission for $\varepsilon=500, \mathrm{~dB}$ & -6.8 \\
\hline maximum electric field in ferroelectric for $\varepsilon=400, \mathrm{kV} / \mathrm{cm}$ & 36 \\
\hline power losses for $\varepsilon=400$ (tan $\delta=0.004), \mathrm{MW}$ & 7.4 \\
\hline transmission for $\varepsilon=400, \mathrm{~dB}$ & -0.33 \\
\hline
\end{tabular}



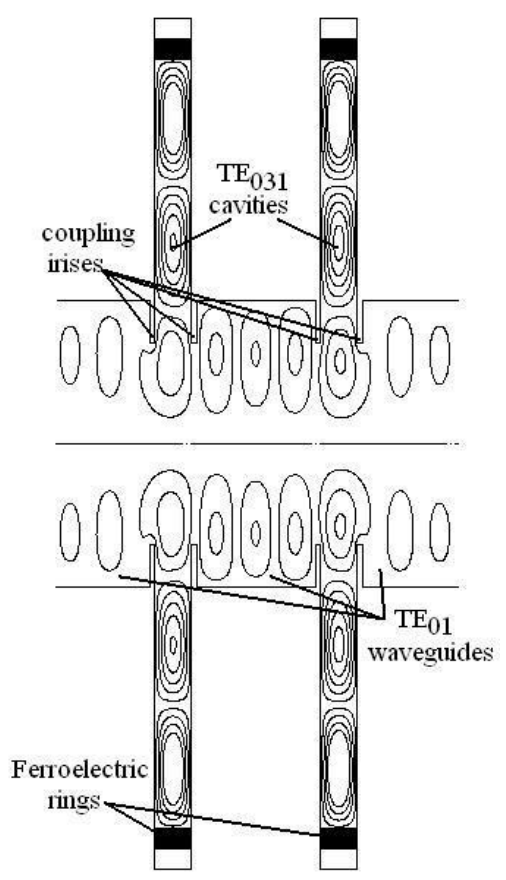

Figure. 6. The initial phase shifter conceptual layout that includes two TE 031 cavities.

One can see from the above equation that, for a given material and power, the single practical way to decrease the RF field is to increase the volume of a ferroelectric element. Note that an increase of the number of ferroelectric cavities in the switch decreases the field, but increases the total RF losses, and utilization of more than two cavities is impractical. On the other hand, the length of the ring is limited by the risk of excitation of modes with higher longitudinal wave number. The maximum possible length is thus about $8 \mathrm{~mm}$. The ring thickness is limited by the excitation of the ferroelectric mode that will lead to strong field increase. Numerical calculations show that the maximum value of thickness is about $1 \mathrm{~mm}$. Thus, the only remaining way to decrease the field is to increase the ring diameter. If the ring diameter is two times higher than in the initial version of the switch cavity, estimations based on the above equation give a maximum field of $24 \mathrm{kV} / \mathrm{cm}$; this value is acceptable from the point of view of possible nonlinear effects. In this case the operating mode in the switch cavity is $\mathrm{TE}_{061}$. It should be noted that, for a ring thickness of $1.13 \mathrm{~mm}$, the ferroelectric modes are close to the operating "vacuum" mode, giving rise to a field increase up to $35 \mathrm{kV} / \mathrm{cm}$.

The switch optimized parameters for the increased ring diameter are given in Table V. The conceptual ferroelectric switch arrangement and dimensions are shown in Figure 7.

The modified switch contains two $\mathrm{TE}_{061}$ switch cavities, each with an internal ferroelectric ring. The distance between the matching diaphragm and the first switch cavity is 22 $\mathrm{mm}$. Note that in calculation the loss tangent is 0.004 , as was achieved for the best samples at 35 $\mathrm{GHz}$. However, the loss tangent for different samples varies from 0.004 up to 0.012 [23], and the average value is about 0.008 , about two times higher. Even in this case the overall efficiency of the pulse compressor is lowered to $\sim 62.5 \%$, which is still higher than the efficiency of a passive compressor with compression ratio $n$ of 10 that would have a total efficiency of $50 \%$. 
Table V. Parameters of the ferroelectric switch with increased diameter

\begin{tabular}{|c|c|}
\hline operating frequency, $\mathrm{GHz}$ & 34.272 \\
\hline number of cavities & 2 \\
\hline cavity operating mode & $\mathrm{TE}_{061}$ \\
\hline cavity length, mm & 8 \\
\hline coupling iris diameter, mm & 10 \\
\hline coupling diaphragm thickness, mm & 1 \\
\hline ferroelectric ring length, mm & 8 \\
\hline ferroelectric ring inner diameter, mm & 64.3 \\
\hline ferroelectric ring thickness, mm & 0.95 \\
\hline waveguide diameter, mm & 18 \\
\hline waveguide mode & $\mathrm{TE} 01$ \\
\hline distance between cavities, $\mathrm{mm}$ & 22 \\
\hline transmitted power, $\mathrm{MW}$ & 100 \\
\hline maximum electric field in ferroelectric for $\varepsilon=500, \mathrm{kV} / \mathrm{cm}$ & 23 \\
\hline power losses for $\varepsilon=500$ (tan $\delta=0.004), \mathrm{MW}$ & 7.8 \\
\hline transmission for $\varepsilon=500, \mathrm{~dB}$ & -6.5 \\
\hline maximum electric field in ferroelectric for $\varepsilon=400, \mathrm{kV} / \mathrm{cm}$ & 26 \\
\hline power losses for $\varepsilon=400(\tan \delta=0.004), \mathrm{MW}$ & 8.3 \\
\hline transmission for $\varepsilon=400, \mathrm{~dB}$ & -0.39 \\
\hline bias voltage, $\mathrm{kV}$ & $\sim 40$ \\
\hline
\end{tabular}

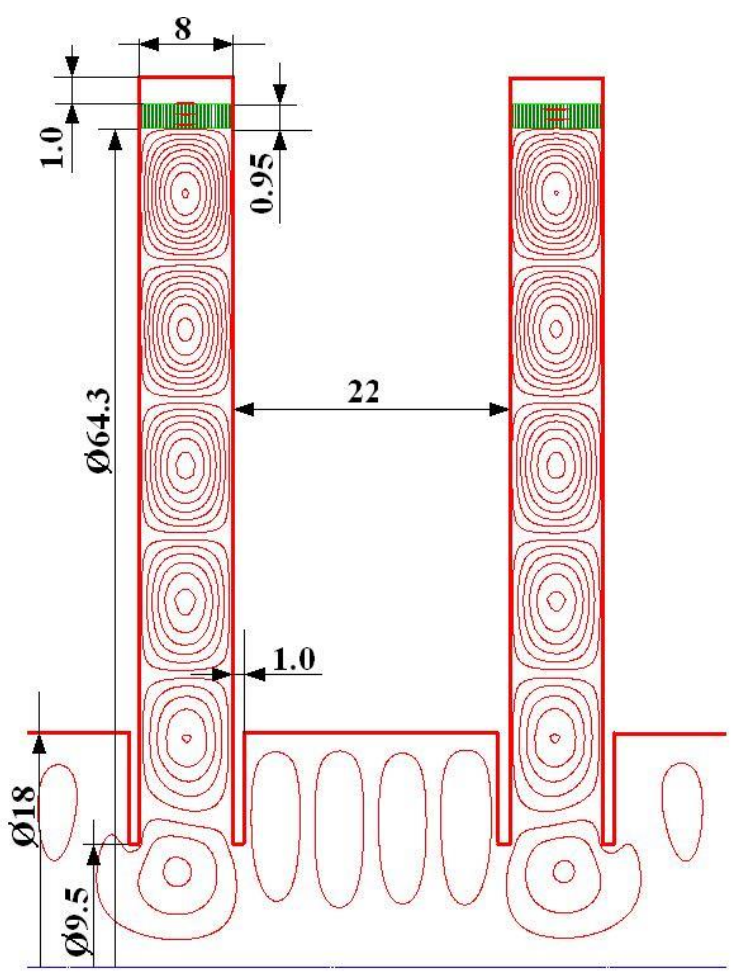

Figure 7. Modified switch conceptual layout and dimensions in mm. 
The frequency dependences of transmission for the energy storage and energy extraction regimes for the entire system that includes the matching diaphragm and the two switch cavities are shown in Figure 8 . These regimes are characterized by the respective values of relative dielectric permittivity $\varepsilon$ for the ferroelectric material, in this case $\varepsilon=400$ and $\varepsilon=500$. The blue curve in Fig. 8 corresponds to the energy storage regime, when both switch cavities have resonance frequencies of about $34.15 \mathrm{GHz}$, and a transmission coefficient of $-6.5 \mathrm{~dB}$ is obtained, when the system is optimized for the most efficient energy storage for compression ratio of 10. In the energy extraction regime, a pulsed bias voltage of $40 \mathrm{kV}$ is applied to the ferroelectric resulting causing a decrease of ferroelectric permittivity from 500 to 400 that leads to the resonance frequency increase. At the permittivity value of 400 the resonance frequencies of the switch cavities are close to the operating frequency (red curve). At resonance, the switch is open, thus providing fast extraction of the energy, and consequently, the desired peak power multiplication. Note, that the switch bandwidth is about $130 \mathrm{MHz}$, which allows the switching time to be less than $8 \mathrm{nsec}$, short enough compared with the compressed pulse width to be acceptable.

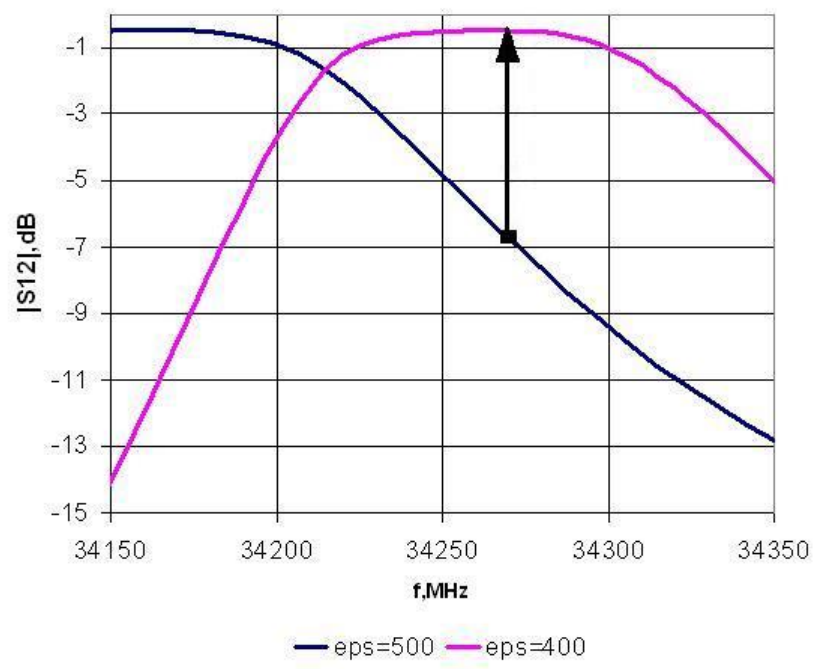

Figure 8. Transmission coefficient $\left|S_{12}\right| v s$. frequency for $\varepsilon=500$ (blue curve), and $\varepsilon=400$ (red curve).

The concept for biasing the ferroelectric in the switch cavity is shown in Figure 9a. The biasing in this case is transverse with respect to the RF electric field. The RF field distribution for the operational mode $\mathrm{TE}_{06 n}$ allows one to separate one cavity wall from the rest of the cavity by circular non-radiating slots $4 \mathrm{~mm}$ in width practically without distortion of the surface RF currents. The DC bias electric field distribution on the cavity surface near the field maximum is shown in Figure 9b. One can see that the electric field doesn't exceed $120 \mathrm{kV} / \mathrm{cm}$. For pulse lengths shorter than about $1 \mathrm{~ms}$, an empirical relation [22] for the high voltage breakdown condition is given by $E_{S} V \leq 1000 \tau^{-0.34}$, where $E_{S}$ is surface electric field on the bias electrode in $\mathrm{kV} / \mathrm{cm}, V$ is the voltage in $\mathrm{kV}$, and $\tau$ is pulse width in sec. For $\tau=1 \mu$ s and $V=40 \mathrm{kV}$, the maximum surface electric field $E_{S}$ for this design, namely $120 \mathrm{kV} / \mathrm{cm}$, well satisfies this criterion. Note, that due to the high permittivity of the ferroelectric the bias field is essentially uniform inside the ferroelectric. 


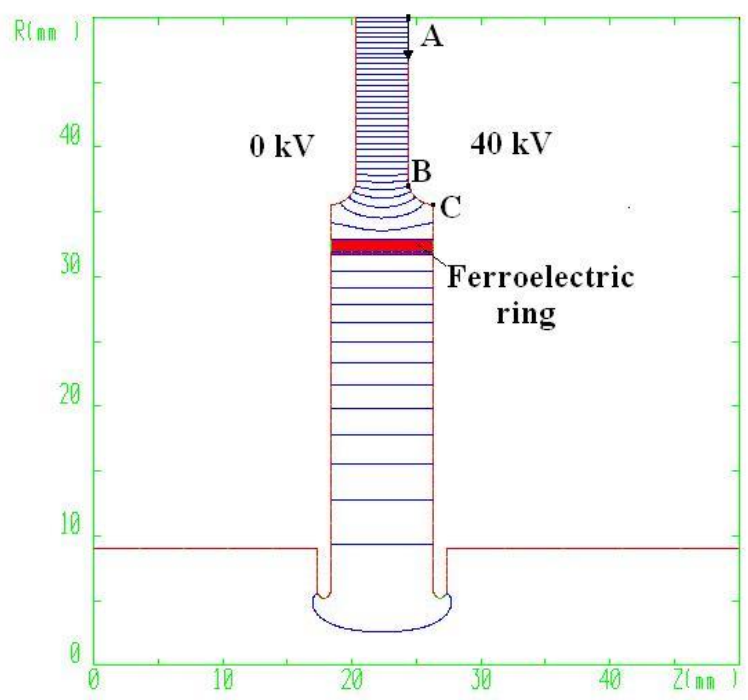

(a)

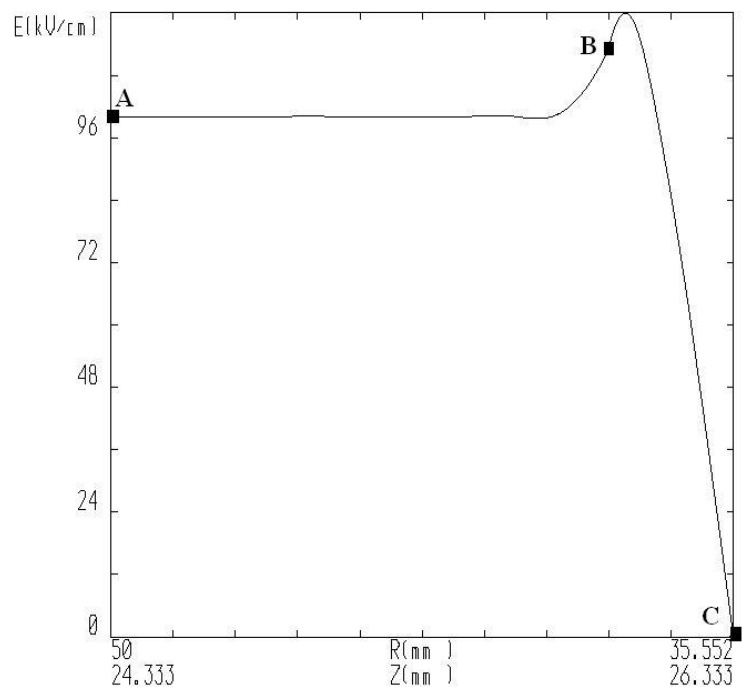

(b)

Figure 9. (a) Concept for biasing of the switch cavity, and (b) distribution of DC bias electric field along the cavity surface from point $\mathbf{A}$ to point $\mathbf{C}$.

The RF field pattern for the realistic switch cavity with biasing slots is shown in Figure 10, a 3-D model of the cavity is shown in Figure 11, and a plot of the magnitude of RF electric field in the median plane of the cavity is shown in Figure 12. Note that the RF field decays exponentially in the upper circular slot and practically vanishes in the side wall slot. This RF field behavior prevents any significant RF from leaking out through these slots.

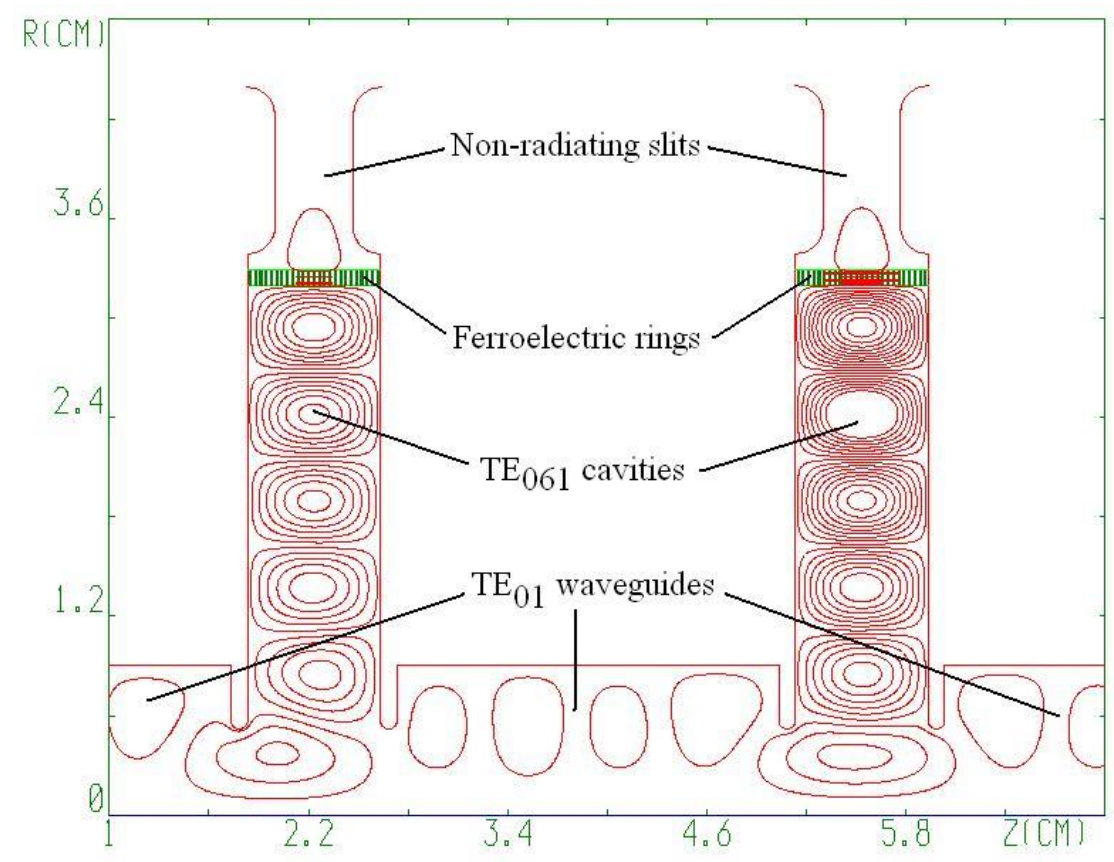

Figure 10. RF field pattern in the switch cavity with circular non-radiating slots. 


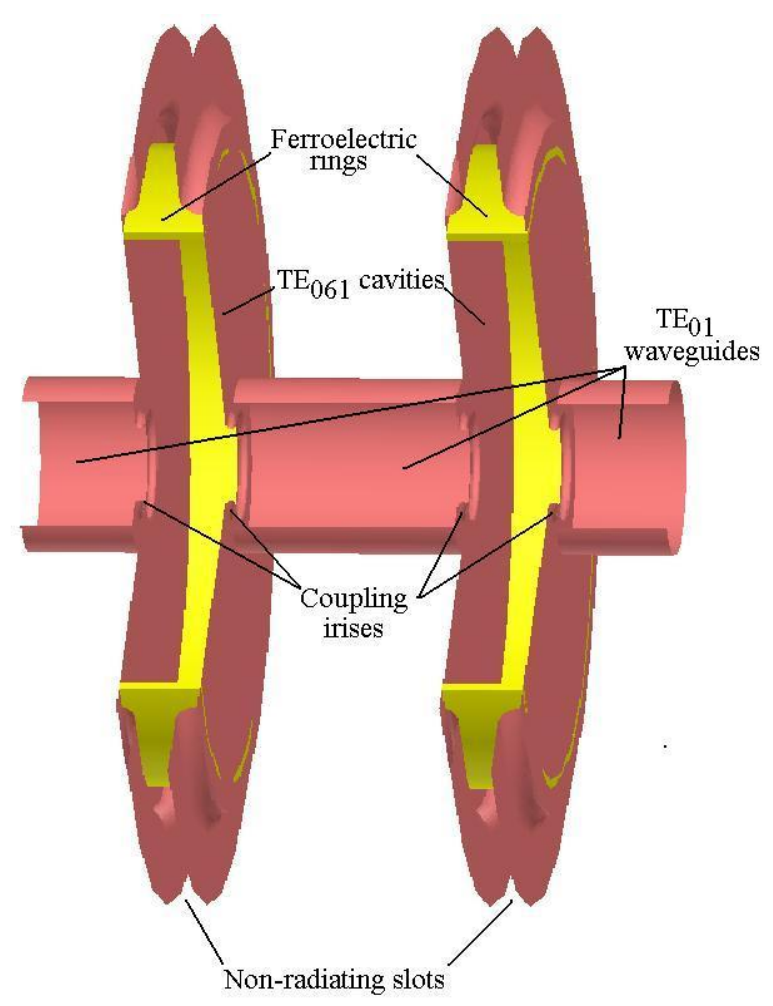

Figure 11. A 3-D model of the switch.

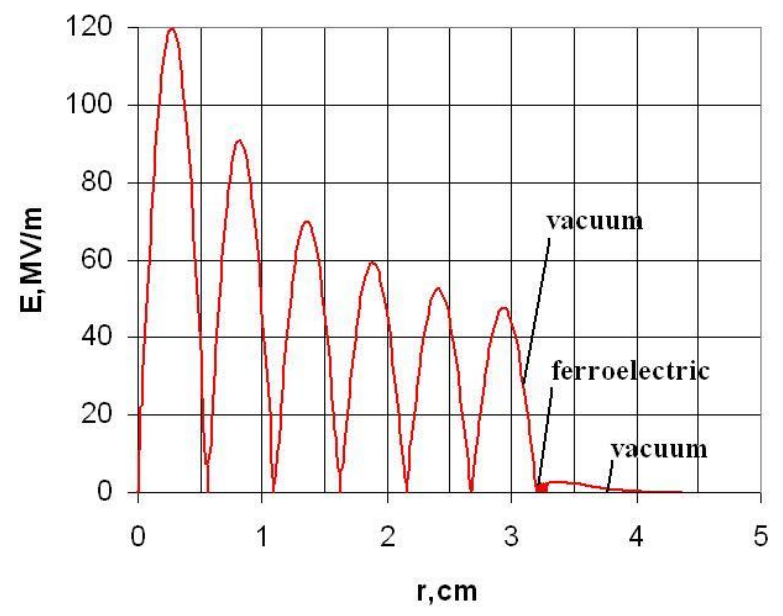

Figure 12. Electric field $v s$. radius in the median plane of the $\mathrm{TE}_{061}$ cavity shown in Fig. 10. Note the relatively small field in the region of the ferroelectric $(3.2<r<3.3 \mathrm{~cm})$, and the strong field decay in the upper circular biasing slot $(r>3.3 \mathrm{~cm})$.

In Figure 13, a SolidWorks drawing is shown of the X-band ferroelectric phase shifter [10] designed by AES, Inc. for Omega-P, Inc., within the scope of DoE SBIR grant DE-FG0203ER83739. The design may demonstrate the idea of the practical arrangement for Ka-band 
ferroelectric switch. One can see the vacuum vessel, high-voltage feed-thru and biasing distribution circuit, and RF circuit with ferroelectric rings. The Ka-band switch would be designed along similar lines, except for use of two cavities in place of four, and for a scaling down by a factor-of-three in critical dimensions because of the higher frequency.

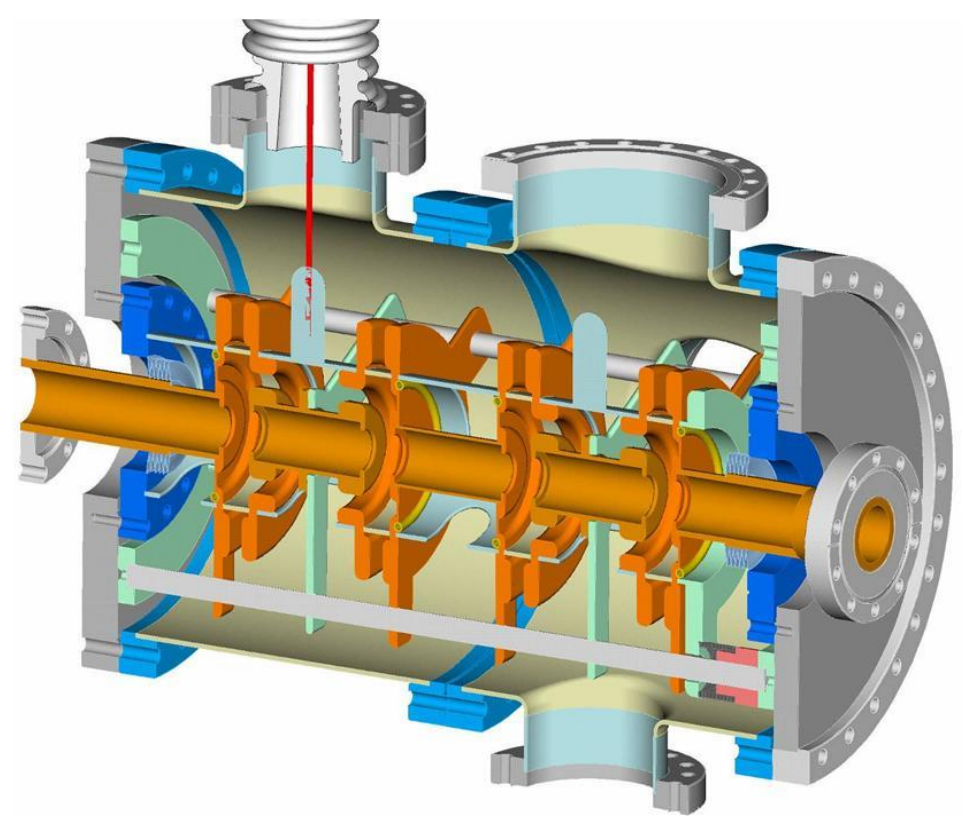

Figure 13. Design of the X-band ferroelectric phase shifter made for Omega-P by AES.

\section{IIIe. Ferroelectric materials}

Euclid Techlabs LLC, working under a sub-grant from Omega-P, successfully sintered a sample ferroelectric ring with a diameter of about $34 \mathrm{~mm}$ and thickness of $2 \mathrm{~mm}$ (as seen in Fig. 12). This was considered to be the main initial goal for Euclid. This bulk ferroelectric is based on a composition of BST ceramics, magnesium compounds, and rare-earth metal oxides. This new ferroelectric $[23,24]$ has a permittivity, dielectric loss factor, and tunability required for the switch designs described above.

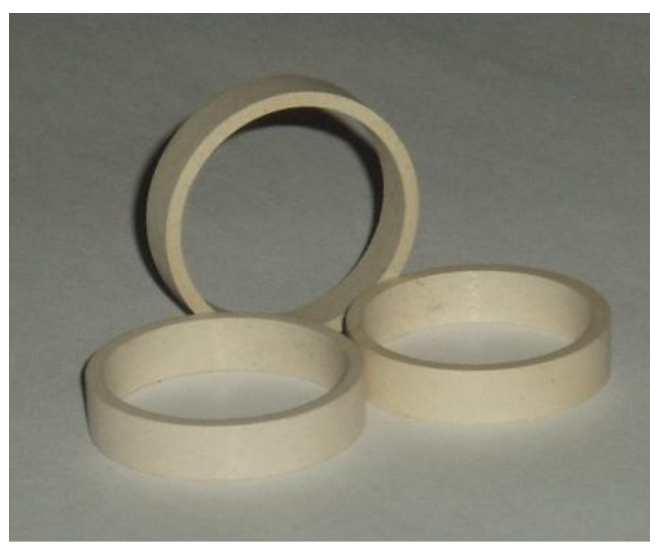

Fig. 12. The ferroelectric ring with a diameter of $34 \mathrm{~mm}$, length $7 \mathrm{~mm}$ and thickness of $2 \mathrm{~mm}$. 
The new design of the switch demands, however, the ring diameter of $64.3 \mathrm{~mm}$. During the work on X-band phase shifter, Euclid Techlabs developed technology and sintered the ring having even bigger diameter of $106 \mathrm{~mm}$ but thickness of $2.8 \mathrm{~mm}$ (see Fig. 13) made of the same material. However, manufacturing of the ring having required thickness of $0.95 \mathrm{~mm}$ may be a serious technological challenge even for smaller diameter of $64.3 \mathrm{~mm}$. The main problem is to form sharp edges of the ring, and flat end surface in order to provide further metallization of these ring ends strong enough. Further, Euclid Techlabs also developed ferroelectric pipes of small thickness of $0.6 \mathrm{~mm}$, as shown in Fig. 14.

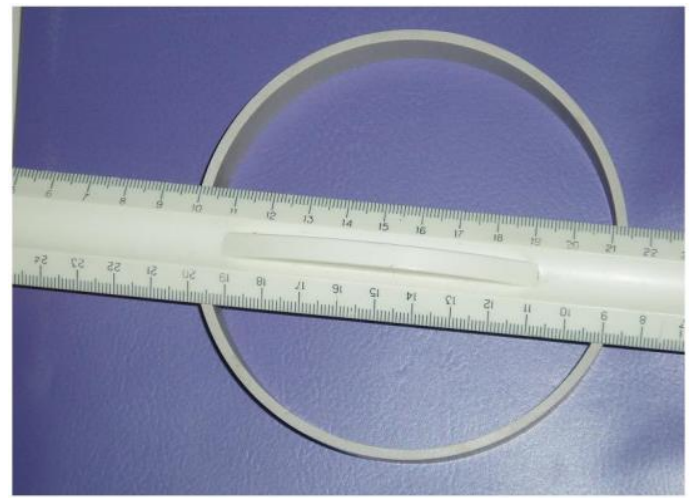

Fig. 13. A ferroelectric ring with a diameter of $106 \mathrm{~mm}$, a thickness of $2.8 \mathrm{~mm}$ and a length of $20 \mathrm{~mm}$.

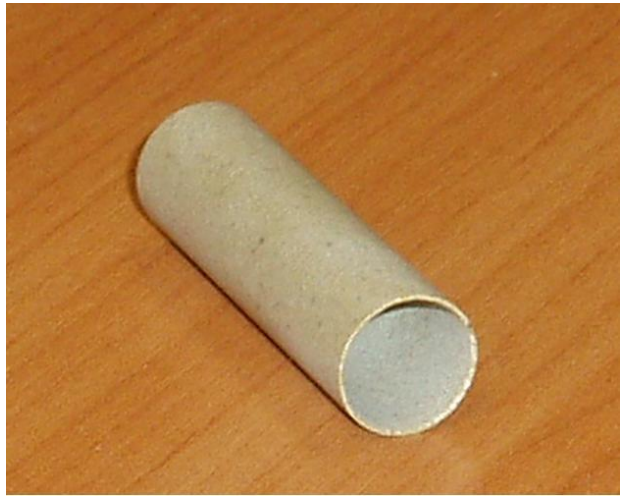

Fig. 14. Small ground pipe made of the same material as the ring shown in Fig. 11, having a wall thickness of $0.6 \mathrm{~mm}$.

Investigations were carried out with three compositions of $\left(\mathrm{Ba}_{\mathrm{x}} \mathrm{Sr}_{1-\mathrm{x}}\right) \mathrm{TiO}_{2}$ with additives of various compounds; these varieties are here labeled BSM1, BSM2 and BSM+. Tests on these samples showed that it is possible to produce low-loss ferroelectric elements with a relative dielectric permittivity $\varepsilon$ in the range of 400 to 500. At this level of permittivity the ratio of barium and strontium components in the solid solution is within the range $x=0.4 \ldots 0.6$, with the addition of magnesium compounds. Tunability of the permittivity $\varepsilon$ by application of a DC electric field was measured at frequencies of both $1 \mathrm{MHz}$ and $10 \mathrm{GHz}$. Measurements showed that the tunability factor is up to $22 \%$ for BSM1 ceramic and up to $20 \%$ for BSM2 and BSM+ at applied transverse biasing fields of $45-50 \mathrm{kV} / \mathrm{cm}$. The loss tangent $\tan \delta$ of the samples was measured over a wide range of frequency from 3 to $35 \mathrm{GHz}$ as well as at the low frequency of 1 MHz. A $\tan \delta$ value of about $4 \times 10^{-3}$ was found at $35 \mathrm{GHz}$ for the best sample.

However, the loss tangent for different samples varies from 0.004 up to 0.012 , and further work is required in order to achieve a good yield for samples with minimal loss tangent. Note, that in order to achieve mechanical properties necessary for the ring of small thickness, the material is to be modified that, in turn, require the new experiments necessary to adjust the material compound in order to achieve required electrical and RF properties.

Further work involved an investigation of the possibility of developing ferroelectric material based on barium strontium titanium oxides solid solution having tunability $>20 \%$ at 50 $\mathrm{kV} / \mathrm{cm}$, but lower dielectric constant (up to 300). The lower dielectric constant allows increase of the ring thickness. This will make in turn easier the ring manufacturing and decreases the electric field and losses in the ferroelectric rings. This challenging task can be accomplished by experimental synthesis and data analysis of the recently developed $(\mathrm{Ba}, \mathrm{Sr}) \mathrm{TiO}_{3}$ ferroelectrics with increased $\mathrm{Mg}$-content additives concentration (more than 50\%). Initial studies showed that 
this Mg-based additives concentration increasing allows to low the dielectric constant value down to 400 300 at the same level of the bulk tunability of the sample for a given bias field magnitude. The dielectric value of 400 has been demonstrated by Euclid, as seen in Table VI (loss tangent measurements were done at $8.5 \mathrm{GHz}$ ).

Table VI. Dielectric properties of samples of

(1-y) $\left\{\mathrm{xBaTiO}_{3}-(1-\mathrm{x}) \mathrm{SrTiO}_{3}\right\} \cdot \mathrm{yMg}$ compounds.

\begin{tabular}{|c|c|c|c|c|c|c|}
\hline \multicolumn{2}{|c|}{ Composition } & \multicolumn{4}{|c|}{ RF parameters } & \multirow{2}{*}{$\begin{array}{c}Q \times f \\
\mathrm{GHz}\end{array}$} \\
\hline $\mathrm{X}$ & $\mathrm{y}$ & $\mathrm{T}_{\mathrm{c}},{ }^{\mathrm{o}} \mathrm{K}$ & $\varepsilon$ & $\begin{array}{r}\tan \delta \\
\times 10^{4}\end{array}$ & tunability & \\
\hline 0.45 & 0.15 & 170 & 462 & 1.8 & $5 \%$ at $19.3 \mathrm{kV} / \mathrm{cm}$ & 1600 \\
\hline 0.45 & 0.20 & 172 & 401 & 1.3 & $3 \%$ at $20 \mathrm{kV} / \mathrm{cm}$ & 2900 \\
\hline 0.50 & 0.15 & 191 & 455 & 1.2 & $8 \%$ at $20 \mathrm{kV} / \mathrm{cm}$ & 1500 \\
\hline 0.50 & 0.20 & 185 & 480 & 1.2 & $5 \%$ at $19.5 \mathrm{kV} / \mathrm{cm}$ & 1900 \\
\hline 0.50 & 0.25 & 185 & 420 & 1.1 & $6 \%$ at $20 \mathrm{kV} / \mathrm{cm}$ & 2300 \\
\hline 0.55 & 0.15 & 208 & 660 & 2.4 & $9 \%$ at $19.5 \mathrm{kV} / \mathrm{cm}$ & 1300 \\
\hline 0.55 & 0.20 & 211 & 520 & 1.6 & $12 \%$ at $19.5 \mathrm{kV} / \mathrm{cm}$ & 1400 \\
\hline 0.55 & 0.25 & 208 & 516 & 3.0 & $12 \%$ at $19.5 \mathrm{kV} / \mathrm{cm}$ & 1300 \\
\hline 0.60 & 0.20 & 224 & 760 & 5.5 & $11 \%$ at $20 \mathrm{kV} / \mathrm{cm}$ & 800 \\
\hline
\end{tabular}

Table VI shows that the tunability increases for the higher barium content BSM (BST-MgO) compositions: for $\mathrm{x}=0.45$ the tunability is $3-8 \%$ while for $\mathrm{x}=0.55-0.60$ it reaches $11-12 \%$. It should be noticed that dielectric constant value of $\sim 400$ have been demonstrated for the low barium content compositions of $\mathrm{x}=0.45$. At the same time, barium content increasing causes dielectric constant value raise from 400 to 700, see Table VI. It was also found that the $\mathrm{Mg}$ content additives weakly interact with the main BST composition. Preliminary studies showed that this dielectric constant raise can be compensated by introducing additional $\mathrm{MgO}$ and other linear ceramic additives in the range of $45-50 \%$ of the overall ferroelectric ceramic content while keeping the same high tunability value of the BST-MgO solid solution. Theoretical analysis of the composite dielectric tunability showes good agreement with the recent experimental results for the BST compositions with relatively low linear ceramic component content [25]. The ferroelectric ceramic with the barium content in the range of $x=0.55-0.60$ and low dielectric constant value of 300-400 was studied experimentally during the project. 


\section{IIIf. Tolerance analysis}

According to Fig. 6, in the energy storage regime $\left|\mathrm{S}_{12}\right|$ changes from $-5.6 \mathrm{~dB}$ to $-7.6 \mathrm{~dB}$ within a frequency interval of $\pm 13 \mathrm{MHz}$ at $34.27 \mathrm{GHz}$ while, according to Fig. 3 and Table II, the total efficiency of the pulse compressor decreases by no more than $1 \%$. Thus, to maintain a precision in power gain within these limits, the switch should be tuned with an accuracy better than $\pm 13 \mathrm{MHz}$. In Table VI the resonant frequency tolerances of the switch are shown in terms of variations of the geometrical parameters; these parameters are defined in Fig. 15. One can see that the switch cavity does not require a manufacturing precision much better than about $25 \mu \mathrm{m}$ to maintain the $\pm 13 \mathrm{MHz}$ tolerance. Final tuning of the cavity will be done by changing the depth of the groove located on the end wall opposite to the side wall slot. The procedure of the ferroelectric ring final grinding was developed in order to get required switch cavity spectrum that allows avoid ferroelectric resonances in vicinity of the operation frequency, and thus, electric field enhancement in the ferroelectric rings. A special test cavity will be manufactured that will be used in the process of the ring manufacturing. The ferroelectric ring will be ground initially in order to achieve initial dimensions (internal diameter and thickness) determined basing on the measurements of the witness sample that is sintered together with the ring and is made of the same material. After that the ring will be inserted into the test cavity, and the cavity spectrum will be measured. Based on these measurements, the final ring dimensions will be determined in order to get correct frequencies of the operating mode and ferroelectric modes the same time, and the ring dimensions will be finally corrected.

Table VII. Resonant frequency tolerances.

\begin{tabular}{|c|c|c|}
\hline geometrical parameter & $\begin{array}{c}\text { nominal } \\
\text { value, } \mathrm{mm}\end{array}$ & $\begin{array}{c}\text { derivation } \\
\mathrm{MHz} / \mu\end{array}$ \\
\hline internal diameter of the ferroelectric ring, $\mathrm{D}_{\mathrm{r}}$ & 63.9 & $\mathrm{df} / \mathrm{d} \mathrm{D}_{\mathrm{r}}=-0.33$ \\
\hline ferroelectric ring thickness, $\mathrm{H}$ & 0.95 & $\mathrm{df} / \mathrm{dH}=-1.0$ \\
\hline cavity length, $\mathrm{L}_{\mathrm{c}}$ & 8.0 & $\mathrm{df} / \mathrm{dL}_{\mathrm{c}}=-1.0$ \\
\hline cavity diameter, $\mathrm{D}_{\mathrm{c}}$ & 67.8 & $\mathrm{df} / \mathrm{dD}_{\mathrm{c}}=-0.004$ \\
\hline coupling iris diameter, $\mathrm{D}_{\mathrm{a}}$ & 10.4 & $\mathrm{df} / \mathrm{dD} \mathrm{a}=-0.065$ \\
\hline coupling diaphragm thickness, $\mathrm{T}$ & 1 & $\mathrm{df} / \mathrm{dT}=+0.03$ \\
\hline non-radiating slot width, $\mathrm{Lg}$ & 4.0 & $\mathrm{df} / \mathrm{dL} \mathrm{g}=-0.003$ \\
\hline
\end{tabular}

Fig. 15. The switch cavity layout and geometrical parameters. 


\section{IIIg. Engineering design concept for the high-power switch}

A design concept for the switch that would be suitable for high-power use is shown in Fig. 16. The two cavities are located in a high-vacuum chamber that is external to the highvacuum waveguide. In order to apply biasing voltages up to $40 \mathrm{kV}$, part of the inner end wall in each cavity is separated from the rest of the cavity by a circular non-radiating slot. Note that an additional small DC bias voltage (a few $\mathrm{kV}$ ) may be applied, if necessary, in order to suppress multipactoring near the ferroelectric rings and provide fine cavity tuning within a few MHz. Water cooling is not shown, though it may be desirable even for the tests with magnicon, having power of up to $40 \mathrm{MW}$ in pulses of $<1 \mu$ s duration at a repetition rate of up to $10 \mathrm{~Hz}$.

As is known, [13] the switching time in ferroelectrics is very short and limited not by intrinsic ferroelectric properties, but by the time required for build-up of the biasing voltage. This built-up time is limited by the capacitance of the ferroelectric rings. In the present case the overall capacitance of two rings is about $200 \mathrm{pF}$. Thus, in order to obtain a switching time of less than $10 \mathrm{~ns}$ required for pulse compressor operation, the impedance of the biasing generator for both switches (capacitance is $400 \mathrm{pF}$ ) must be less than 25 Ohms.

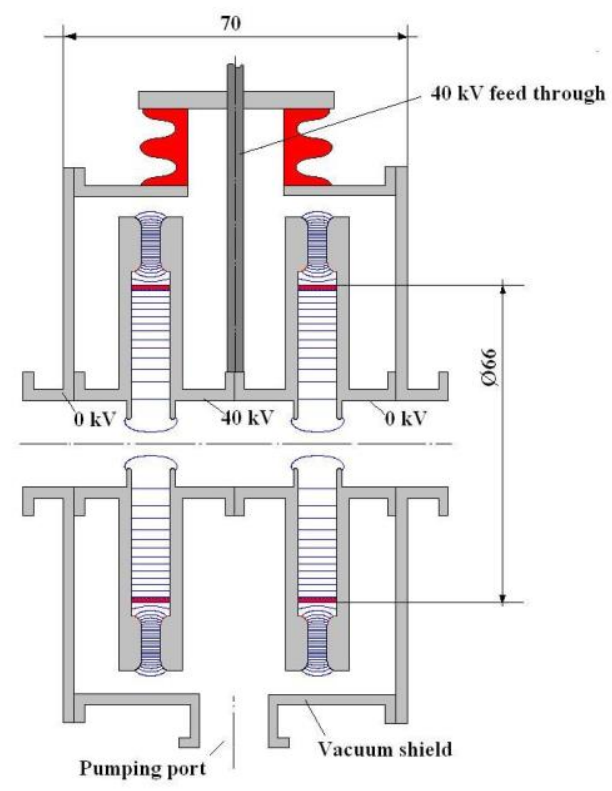

Fig. 16. A conceptual arrangement of the ferroelectric switch, showing the bias electric field lines. Dimensions are in $\mathrm{mm}$.

\section{IIIh. Experimental test setup}

The new switch was designed for installation at the Yale/Omega-P 40-MW Ka-band magnicon test facility. The arrangement of the switch tests at half of the magnicon output power, i.e., at 10-20 MW is shown in Fig. 17. Because the magnicon has four outputs with nearly equal power [2], a power combiner is needed to feed the switch. The $\mathrm{Ka}$-band power combiner capable to operate with the full design magnicon power of $40 \mathrm{MW}$ is developed and built at Omega-P [26], the photo is shown in Fig. 18. The circuit also requires high-power windows, mode converters from WR-28 waveguide to circular waveguide in the $\mathrm{TE}_{01}$ mode, and high power load. All this equipment is developed and built [26,27]. The photo of the high-power Ka- 
band load operating at the $\mathrm{TE}_{01}$ mode is shown in Fig. 19. Note that the circuit requires highpower $3 \mathrm{~dB}$ hybrid coupler in order to protect magnicon of the reflections from the switch, when it operates in energy storage regime. The coupler is to be developed and built during the Phase II of the project. In fact, it is the fortuitous availability of these high-power components that obviates need for time-consuming design and construction of expensive new structures, thus allowing the project to be carried out within the limited time and budget of Phase II. Arrangement of the Ka-band pulse compressor using the ferroelectric switch is shown in Fig. 20.

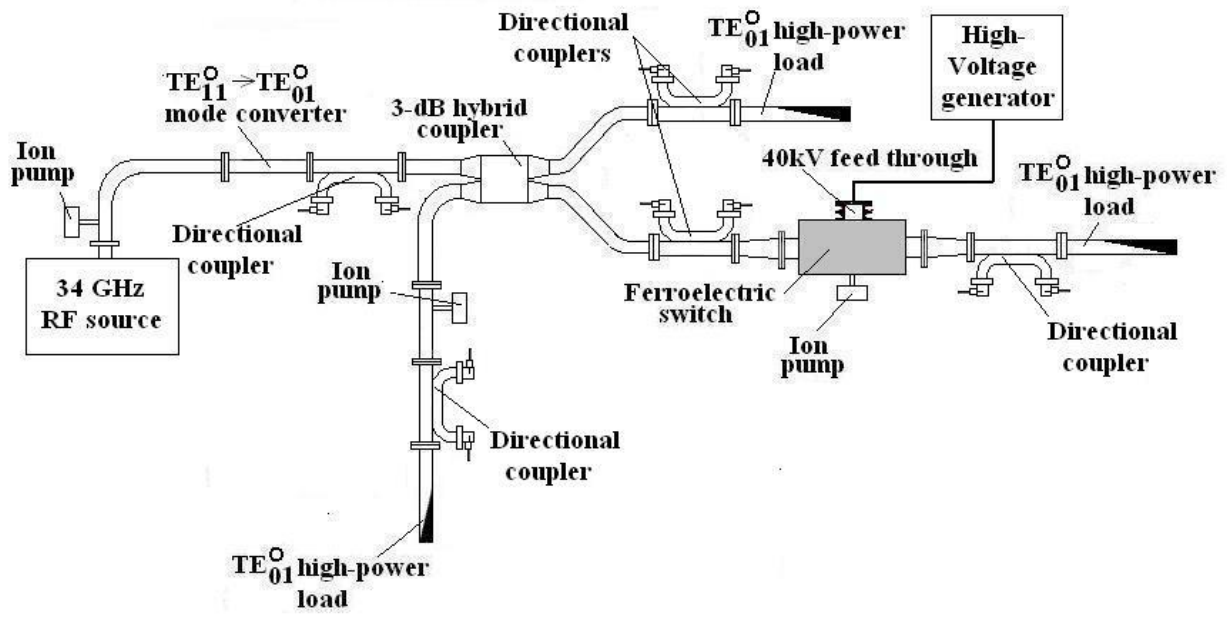

(a)

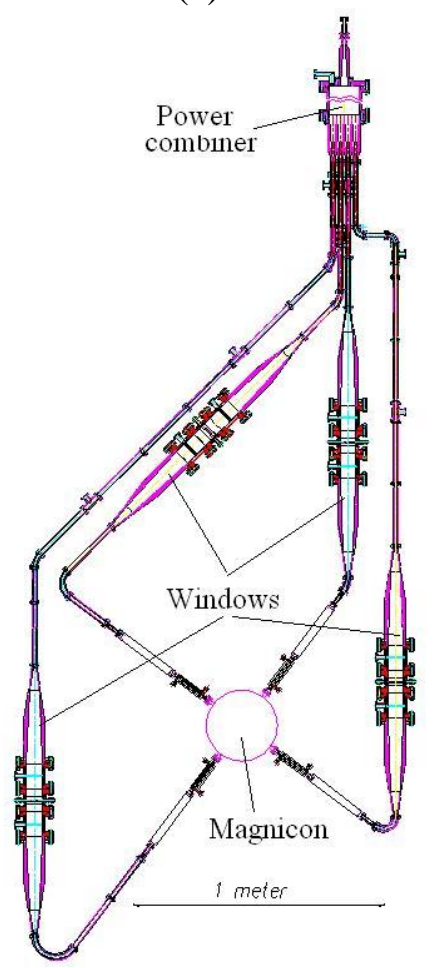

b)

Fig. 17. Experimental setup for the ferroelectric switch tests up to half of the output magnicon power. Simplified arrangement of the magnicon and power combiner is shown (a). Arrangement of the $34 \mathrm{GHz}$ RF source that includes magnicon, power combiner, windows and mode converters (b). 


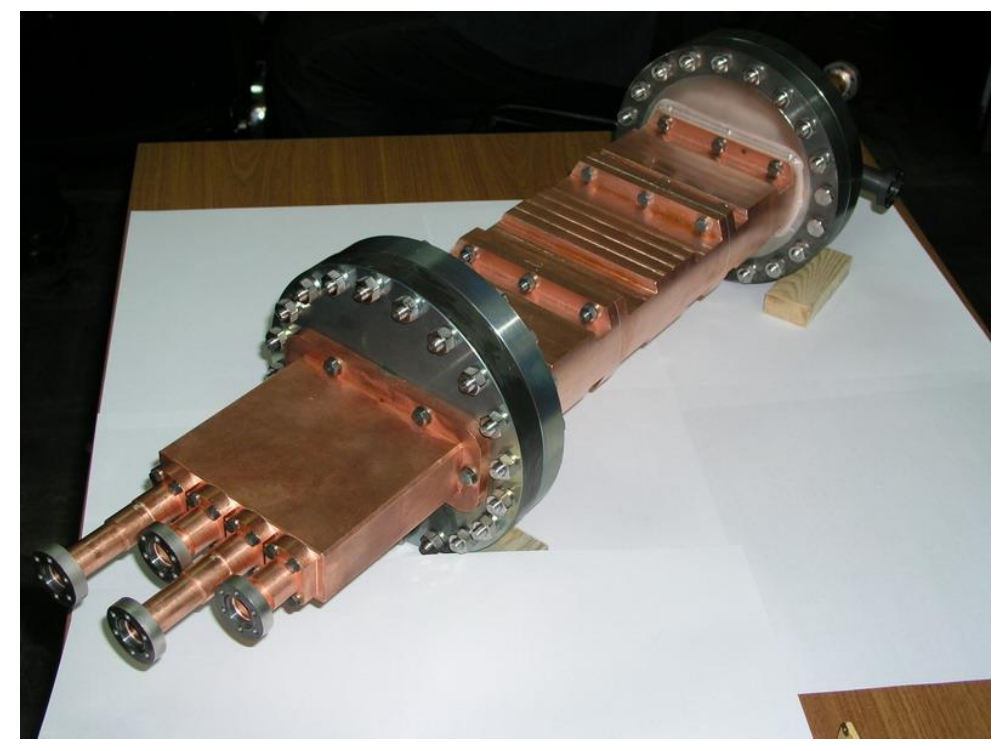

Fig. 18. Photo of the high power Ka-band power combiner having four inputs, and one output.

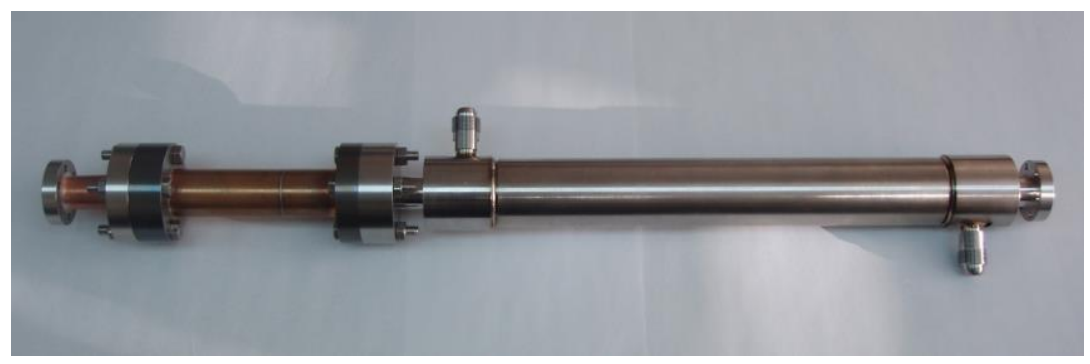

Fig. 19. Photo of the load with $\mathrm{TE}_{11}-\mathrm{TE}_{01}$ mode converter at the left side.

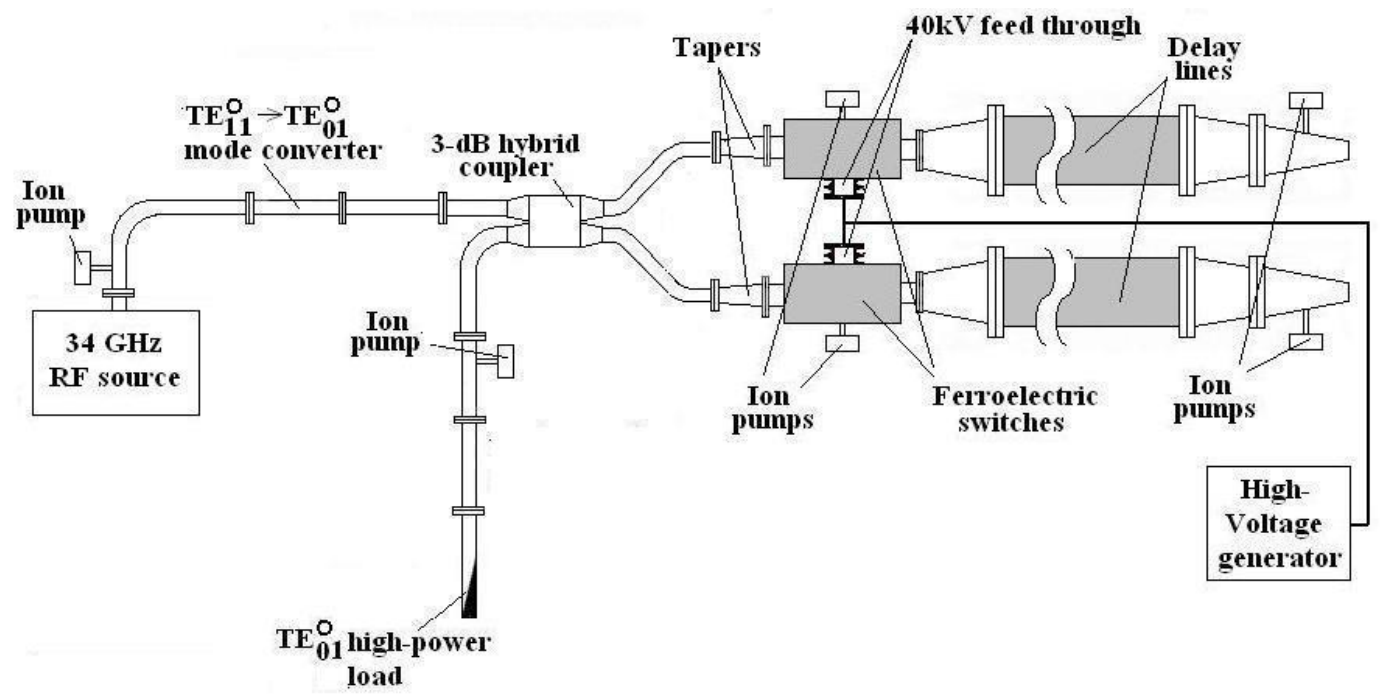

Fig. 20. Arrangement for testing the active pulse compressor with ferroelectric switch. 
Further details on this project may be found in the Appendices, including theoretical analysis for a cavity partially filled with dielectric material, such as the ferroelectric element used in the switch, copies of published scientific articles on the subject, and copies of informal presentations that contain results of measurements on the prototype X-band ferroelectric switch.

\section{REFERENCES}

1. R.W. Assmann, F. Becker, R. Bossart, et al, "A $3 \mathrm{TeV} \mathrm{e}^{+} \mathrm{e}^{-}$Linear Collider Based On CLIC Technology," CERN 2000-008, 28 July 2000.

2. O.A. Nezhevenko, V.P. Yakovlev, M.A. LaPointe, E.V. Kozyrev, S.V. Shchelkunov, and J.L. Hirshfield, "Status Of 34 GHz, 45 MW Pulsed Magnicon," PAC2005 Proceedings (C. Horak, ed.), Knoxville, May 16-20, 2005, p. 1922-1924.

3. M. Petelin, J. Hirshfield, Yu.Yu. Danilov, S. Kuzikov, V.Pavelyev, D. Schegolkov, and A. Yunakovsky, "Components for Quasi-Optically-Fed Linear Accelerators," RF2005, Kalamata, Greece, June 13-17, 2005, AIP Conference Proc. 807, Melville, N.Y., 2006 p.408.

4. S.G. Tantawi, "New Development in RF Pulse Compression," SLAC-PUB-8582, Aug. 2000.

5. S.G. Tantawi, R.D. Ruth, and A.E. Vlieks, "Active Radio Frequency Pulse Compression Using Switched Resonant Delay Lines,” SLAC-PUB-95-6748, June 1995.

6. S.G. Tantawi, C. Adolphsen, S. Holmes, T. Lavine, et al, "The Next Linear Collider Test Accelerator's RF Pulse Compression and Transmission Systems," SLAC-PUB-7247, February 1999.

7. S.G. Tantawi, R.D. Ruth, A.E. Vlieks, and M. Zolotorev, "Active High-Power RF Pulse Compression Using Optically Switched Resonant Delay Lines," IEEE Trans. On Microwave Theory and Tech., v. 45, No 8, Aug 1997, p. 1486.

8. F. Tamura and S.G. Tantawi, "Development Of High-Power X-Band Semiconductor RF Switch For Pulse Compression Systems Of Future Linear Colliders,” LINAC 2000, pp. 751 753.

9. A.L. Vikharev, O.A. Ivanov, A.M. Gorbachev, V.A. Isaev, S.V. Kuzikov, S.H. Gold, A.K. Kinkead, O.A. Nezhevenko, J.L. Hirshfield, "Active RF Pulse Compressor At $11.4 \mathrm{GHz}$ Using Plasma Switch," PAC2003 Proceedings (J. Chew, ed.), Portland, Oregon, May 2003, pp. 1147-1149.

10. V.P. Yakovlev, O.A. Nezhevenko, and J.L. Hirshfield, "High Power Ferrolelectric Switches at Centimeter and Millimeter Wave Lengths," PAC2005 Proceeding, Knoxville, May 16-20, 2005, p. 2056-2058.

11. V.P. Yakovlev, O.A. Nezhevenko, J.L. Hirshfield, and A.D. Kanareykin, "Ferroelectric Switch For An Active RF Pulse Compressor," RF2003, Berkeley Springs, West Virginia, USA, June 22-26, 2003, AIP Conference Proc. 691, Melville, N.Y., 2003, pp.187-195.

12. L.C. Sengupta, "Bulk Ceramic Ferroelectrics and Composites: Manufacture, Microwave Properties and Applications," IMS2000; see also http://my.ece.ucsb.edu/yorklab/Projects/Ferroelectrics/IMS2000\%20Workshop/WFE002.pdf

13. D. Webb, "Applications of Ferroelectrics in Military Systems," IMS2000; see also http://my.ece.ucsb.edu/yorklab/Projects/Ferroelectrics/IMS2000\%20Workshop/WFE004.pdf

14. E.A. Nenasheva, A.D. Kanareykin, N.F. Kartenko, and S.F. Karmanenko, "Ceramic materials based on $(\mathrm{Ba}, \mathrm{Sr}) \mathrm{TiO}_{3}$ solid solutions for tunable microwave devices," Journal of Electroceramics, v. 13, pp. 235-238, 2004.

15. S.F. Karmanenko, E.A. Nenasheva, A.I. Dedyk, A.D. Kanareykyn and A.A. Semenov, 
"Frequency dependence of microwave quality factor of doped BaxSr1-xTiO3 ferroelectric ceramics," Integrated Ferroelectrics, v. 61, pp. 177-181, 2004.

16. A. Kanareykin, E. Nenasheva, S. Karmanenko, "New Low-Loss Ferroelectric Materials for Accelerator Applications," AAC2004 Proceedings, Stony Brook, June 2004, AIP Conference Proc. 737, Melville, N.Y., 2004, pp.1016-1024.

17. A.L. Vikharev, A.M. Gorbachev, O.A. Ivanov, V.A. Isaev, V.A. Kondakov, S.V. Kuzikov, A.G. Litvak, M.I.Petelin, J.L. Hirshfield, and O.A. Nezhevenko, 'Two-channel Active HighPower X-band Pulse Compressor," AIP Conf. Proc., v. 569, p. 741.

18. O.A. Nezhevenko, V.P. Yakovlev, S.H. Gold, M.A. LaPointe, A.W. Fliflet, A.K. Kinkead, J.L. Hirshfield, "Resent Results From The X-Band Pulsed Magnicon Amplifier," PAC2005 Proceeding (C. Horak, ed.), Knoxville, May 16-20, 2005, p. 1979-1981.

19. S. Kazakov, "Multi-mode delay lines," The $11^{\text {th }}$ KEK-SLAC ISG Meeting on linear colliders, KEK, December 16, 2003. http://lcdev.kek.jp/ISG/ISG11/WG\%20f/9-mode_sled.pdf.

20. Y. Goren, N. Mahale, L. Walling, T. Enegren, G. Hulsey, V.P. Yakovlev, V.M. Petrov, "Analytical and Numerical Analysis of the Nonlinear Effects in Ferrite-Tuned Cavities". PAC1993 Proceeding, Washington, 1993, pp.877 - 879.

21. P. Coleman, F. Brandberry, C. Friedrichs, Y. Goren, G. Hulsey, S. Kwiatkowsky, A. Propp, L. Tailor, L. Walling, J. Averboukh, M. Karliner, V. Petrov, V. Yakovlev, "Status of the SSC LEB RF Cavity", PAC1993 Proceeding, Washington, 1993, pp. 824 -826.

22. "High Voltage Vacuum Insulation," R.V. Latham, ed., Acad. Press, N.Y., 1995, pp. 403-429.

23. E.A. Nenasheva, A.D. Kanareykin, N.F. Kartenko, and S.F. Karmanenko, "Ceramic materials based on (Ba,Sr) $\mathrm{TiO}_{3}$ solid solutions for tunable microwave devices," Journal of Electroceramics, v. 13, pp. 235-238, 2004.

24. S.F. Karmanenko, E.A. Nenasheva, A.I. Dedyk, A.D. Kanareykyn and A.A. Semenov, "Frequency dependence of microwave quality factor of doped BaxSr1-xTiO3 ferroelectric ceramics," Integrated Ferroelectrics, v. 61, pp. 177-181, 2004.

25. A. G. Kolpakov, A. K. Tagantsev, L. Berlyand, A. Kanareykin, "Tunability of Composite Dielectrics," Journal of Electroceramics, 2006 (in press).

26. J. L. Hirshfield, A.A. Bogdashov, A. V. Chirkov, G. G. Denisov, A. S. Fix, S. V. Kuzikov, M. A. LaPointe, A. G. Litvak, D. A. Lukovnikov, V. I. Malygin, O. A. Nezhevenko, M. I. Petelin, Yu. V. Rodin, G. V. Serdobintsev, M. Y. Shmelyov, V. P. Yakovlev, "Transmission Line Components For A Future Millimeter-Wave High-Gradient Linear Accelerator," Proc. Of The NATO Advanced Research Workshop on Quassi-Optical Control of Intense Microwave Transmission, 17-20 February, 2004, Nizhny Novgorod, Russia, NATO Science Series II, Vol. 203, pp.147-163.

27. J.L. Hirshfield, O.A. Nezhevenko, M.A. LaPointe, V.P. Yakovlev, "Technology Developments for a Future Millimeter-Wave High-Gradient Linear Accelerator," Proc. of $29^{\text {th }}$ International Conference on Infrared and Millimeter Waves, Sept. 27-Oct. 1, 2004, Karlsruhe, Germany, pp. $529-530$. 


\section{APPENDIX I. Theory of a cavity partially filled with dielectric.}

Let's consider the cavity partially filled by dielectric with permittivity of $\varepsilon$. If the dielectric constant is changed, the detuning of the cavity is equal to

$$
\chi=\frac{2 \Delta f}{f}=-\frac{\Delta \varepsilon}{2 W} \int_{V \varepsilon}|\vec{E}|^{2} d V,
$$

where $\Delta \varepsilon$ is the dielectric constant change (in $\mathrm{F} / \mathrm{m}$ ), $W$ is the energy stored in the cavity, and $E$ is the electric field in the dielectric. Integral in (1) is calculated over the volume $V_{\varepsilon}$ occupied by dielectric.

If the cavity has the input and output ports (see, for example, Fig. 4, where the two cavities are shown), transition coefficient from the input port to the output port is equal to

$$
T^{2} \equiv\left|S_{12}\right|^{2}=4 \beta^{2} /(1+2 \beta)^{2} \cdot 1 /\left(1+\left(Q_{l} \chi\right)^{2}\right),
$$

where $Q_{l}=Q_{0} /(1+2 \beta), Q_{0}$ is the cavity unloaded quality factor and $\beta$ is the coupling coefficient. If the coupling is strong $(\beta>>1)$,

$$
T^{2} \approx 1 /\left(1+\left(Q_{\imath} \chi\right)^{2}\right.
$$

If dielectric has high enough loss tangent (it is typical for the switch cavities considered in the Proposal), the unloaded quality factor is determined mainly by the losses in the dielectric, i.e.,

$$
Q_{0}=\frac{2 W}{\varepsilon t g \delta \int_{V \varepsilon}|\vec{E}|^{2} d V},
$$

where $\operatorname{tg} \delta$ is dielectric loss tangent. From (1-3) one has

$$
Q_{l} \chi=\frac{\Delta \varepsilon}{\operatorname{ctg} \delta(1+2 \beta)}=\frac{\sqrt{1-T^{2}}}{T} \equiv \alpha
$$

If required transmission is known, it is possible to determine the required coupling from (4). For $\beta>>1$ one has

$$
\beta=\frac{\Delta \varepsilon}{2 \varepsilon t g \delta \alpha}
$$

The power dissipation in the dielectric is equal to

$$
P_{d}=\frac{P_{0}}{\beta}=\frac{P_{0} 2 \varepsilon t g \delta \alpha}{\Delta \varepsilon},
$$

For another hand, 


$$
P_{d}=\frac{1}{2} \omega \varepsilon \operatorname{tg} \delta \int_{V \varepsilon}|\vec{E}|^{2} d V
$$

where $\omega$ is a cyclic frequency. From $(6,7)$ it is possible to find maximal electric $E$ field in the dielectric. For the dielectric ring having rectangular cross section with the $\mathrm{TE}_{0 \mathrm{~nm}}$ standing wave inside one has

$\int_{V \varepsilon}|\vec{E}|^{2} d V=\frac{1}{2} \pi r L h E^{2}$

where $r$ is the ring radius, $L$ is it's length, $h$ is it's thickness. From (6) in this case one has:

$E=\left(\frac{8 \alpha P_{0}}{\omega \Delta \varepsilon \cdot \pi r L h}\right)^{\frac{1}{2}}$

Thus, electric field in the dielectric is determined by the transmitted power, the ring size and change in dielectric constant necessary to achieve required transmission.

\section{Example:}

For the cavity considered in the Chapter $\mathbf{6 c}$ on the $34 \mathrm{GHz}$ ferroelectric switch one has:

Frequency, $f$

Transmitted power, $\mathrm{P}_{0}$

Change in dielectric constant, $\Delta \varepsilon / \varepsilon_{0}$

The ring radius, $r$

The ring length, $L$

The ring thickness, $h$

Transmission change per cavity, $T$
$34.272 \mathrm{GHz}$;

$100 \mathrm{MW}$;

100

$32 \mathrm{~mm}$;

$8 \mathrm{~mm}$;

$0.95 \mathrm{~mm}$;

$-3 \mathrm{~dB}(\alpha=1)$

From (8) one can estimate the maximal electric field: $E=24 \mathrm{kV} / \mathrm{cm}$.

\section{APPENDIX II. Copies of related published papers.}

See below.

\section{APPENDIX III. Copies of related informal presentations.}

See below. 


\section{AIP $\mid$ Proceedings}

\section{Ferroelectric Switch For An Active RF Pulse Compressor}

V. P. Yakovlev, O. A. Nezhevenko, J. L. Hirshfield, and A. D. Kanareykin

Citation: AIP Conference Proceedings 691, 187 (2003); doi: 10.1063/1.1635119

View online: $\mathrm{http}: / / \mathrm{dx}$.doi.org/10.1063/1.1635119

View Table of Contents: http://scitation.aip.org/content/aip/proceeding/aipcp/691 ?ver=pdfcov

Published by the AIP Publishing 


\title{
Ferroelectric Switch For An Active RF Pulse Compressor
}

\author{
V.P. Yakovlev ${ }^{1}$, O.A. Nezhevenko ${ }^{1}$, J.L. Hirshfield ${ }^{1,2}$, and \\ A.D. Kanareykin ${ }^{3}$ \\ ${ }^{1}$ Omega-P, Inc., New Haven, CT 06511, USA, \\ ${ }^{2}$ Yale University, Physics Department, New Haven, CT 06520, USA \\ ${ }^{3}$ Euclid Concepts, LLC, Solon, Ohio 44139, USA
}

\begin{abstract}
Principles and preliminary design for a microwave active pulse compressor using an electrically-controlled ferroelectric switch are presented. The design of an $11.4 \mathrm{GHz}, 500 \mathrm{MW}$ pulse compressor with a pulse width of about $40 \mathrm{nsec}$ and a compression ratio of 10 is described. It is planned to test this compressor using the Omega-P/NRL X-band magnicon.
\end{abstract}

\section{INTRODUCTION}

The current design for the linear collider NLC [1] relies on pulse compression to achieve the high peak rf power levels required to drive the accelerator structures $(\sim 500$ MW in 400 nsec pulses). A number of rf pulse compression systems have been under consideration recently including versions of the Delay Line Distribution System (DLDS), Binary Pulse Compression (BPC), and Resonant Delay Line Pulse Compression System (SLED-II) [2]. The mechanisms upon which these compressors operate are passive, in that no element in the compressor structure has time-dependant properties. The rf pulse compression in all these devices is achieved by $180^{\circ}$ phase switching in klystrons. Common limitations of these systems are their relatively low compression ratio $(\sim 4: 1)$, and their very long runs (100's of $\mathrm{km}$ ) of low-loss vacuum waveguide [2]. Present design of NLC/JLC is based on SLED-II pulse compressor. Schematic of SLED-II is shown in Figure 1 taken from [2].

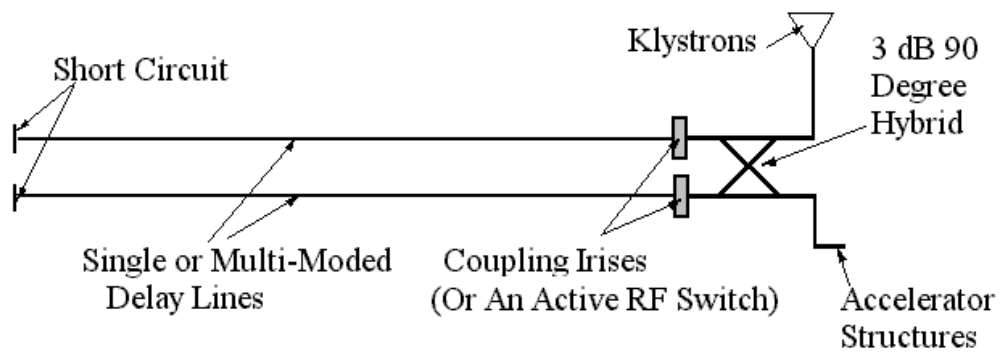

FIGURE 1. Resonant Delay Line Pulse Compression System (SLED-II). The total length of singlemode low-loss delay lines for NLC would be about $180 \mathrm{~km} \mathrm{[2].}$ 
In an attempt to circumvent these limitations, various concepts of active $\mathrm{rf}$ pulse compression have recently received attention, involving switches with optically-varied silicon mirrors [3], PIN/NIP diode arrays [4], and plasmas [5]. To date, none of the tested versions of these active pulse compressors achieved high enough power levels for use with NLC: the maximum output power achieved is $10 \mathrm{MW}$ in the switch based on PIN/NIP diode array active window [6], and $50 \mathrm{MW}$ in the compressor based on plasma switches [7]. Ferromagnetic elements are considered as active elements as well, but only as non-reciprocal components [8] (not as switches), due to their relatively low intrinsic switching speed [9].

In the present paper, an active pulse compressor with a resonance switch based on use of electrically-controlled ferroelectric elements [10] is described. Recently, ferroelectric elements received attention and are already used in a wide range of military and communication devices as fast tunable components [11] because they have the ability to operate to frequencies above $30 \mathrm{GHz}$ with reasonable losses, and have very high tuning speed (intrinsic switching speed is reported to be $\sim 0.01 \mathrm{nsec}$ [12]). These characteristics together with the high electric breakdown strength and good vacuum properties make ferroelectrics an attractive candidate for use in highpower active rf pulse compressors. The rapid switching potential for ferroelectrics could make possible the imposition of coupling changes during energy storage in a SLED-type pulse compressor, thereby allowing efficiency values that exceed the canonical $82 \%$.

\section{GENERAL}

1. Ferroelectrics have an $\mathbf{E}$-field-dependent dielectric permittivity $\varepsilon(\mathbf{E})$ that can be very rapidly altered by application of a bias voltage pulse. The switching time in most instances would be limited by the response time of the external electronic circuit that generates and transmits the high-voltage pulse, and can therefore be in the nsec range.

Ferroelectric materials should have the following properties in order to be used in high-power rf switches for linear collider applications:

- The dielectric constant should not exceed 300-500 to avoid problems in the switch design caused by interference from high-order modes;

- The dielectric constant should change by 10's of percents to provide the required switching properties;

- Bias electric fields should be reasonable, namely a few 10 's of kV/cm;

- The loss tangent should be about $10^{-3}$ or lower at $11 \mathrm{GHz}$ to allow switch operation at high average power in a collider having a repetition rate of $120 \mathrm{~Hz}$.

Modern bulk ferroelectrics, such as barium strontium titanate $\left(\mathrm{Ba}_{\mathrm{x}} \mathrm{Sr}_{1-\mathrm{x}} \mathrm{TiO}_{3}\right.$, or BST) with $\mathcal{E} \sim 500$, have high enough electric breakdown strength $(100-200 \mathrm{kV} / \mathrm{cm})$ and do not require too high a bias electric field $(\sim 20-50 \mathrm{kV} / \mathrm{cm})$ to effect a significant change (20-30\%) in $\mathcal{E}$. Loss tangent for commercially-available samples of these materials is about $\sim 10^{-2}$ at $10 \mathrm{GHz}[11]$.

Euclid Concepts, LLC recently developed and tested a modified bulk ferroelectric based on a composition of BST ceramics, magnesium compounds, and rare-earth metal oxides that has a permittivity $\varepsilon=500$, and $20 \%$ change in permittivity for a bias 
electric field of $50 \mathrm{kV} / \mathrm{cm}$. The loss tangent already achieved for the best samples is less than $4 \times 10^{-3}$ at $35 \mathrm{GHz}$ [13], which corresponds to about $1.3 \times 10^{-3}$ at $11 \mathrm{GHz}$, assuming the well-known linear dependence between loss tangent and frequency [11]. These losses are close to what is required for operation in the NLC/JLC. Development of production techniques for this material continues, with the expectation of further lowering the loss tangent to values of less than $10^{-3}$.

The availability of this ferroelectric already allows one to create an X-band highpower rf switch with the peak power required for the NLC/JLC project.

2. It is planned to use major components from the existing Omega-P two-channel Active Bragg Compressor (ABC-2) [14] to test prototype ferroelectric switches. $\mathrm{ABC}-2$ is currently installed in the X-band magnicon test facility at Naval Research Laboratory, where it is operated for testing switches employing plasma discharge tubes.

In active pulsed compressors with resonance switches, the rf source supplies electromagnetic energy to fill a low-loss storage cavity coupled through an electrically-controlled switch to a load (the accelerating structure). Compressor operation involves two steps: first, that of energy storage when the coupling to the storage cavity is small in order to provide good efficiency of filling; and second, that of energy extraction when the coupling is high to provide fast energy discharge into the accelerating structure. The coupling of the storage cavity with the rf source is controlled by changing the resonance frequency of the switch cavity. In general, it is possible to provide a fast change of resonance frequency of the cavity by rapidly changing the rf properties of an active element situated within the cavity. A schematic diagram of Omega-P's two-channel ABC-2 is shown in Fig. 2. As shown in Fig. 2, two cylindrical $\mathrm{TE}_{01}$-mode storage cavities $(8)$ are each terminated with an adjustable

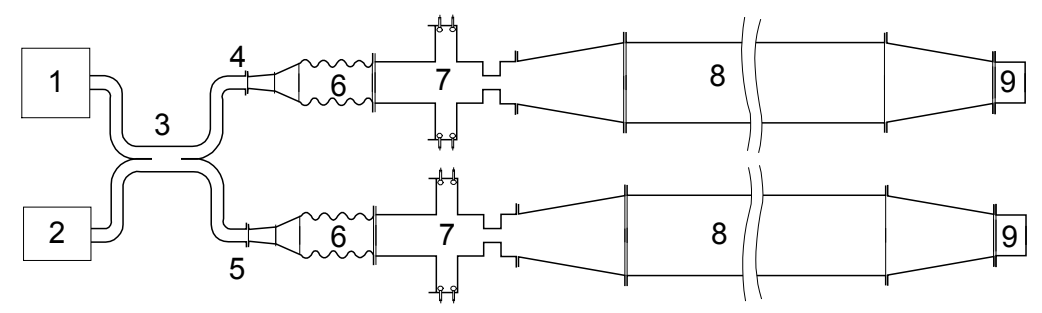

FIGURE 2. Schematic diagram of the two-channel ABC.

short (9) at one end, and an electrically-controlled switch cavity (7) at the other end. Waveguides (4 and 5) are fed from the rf source (1) after the source power is split using a 3-dB hybrid coupler (3). Mode converters (6) transform the mode from $\mathrm{TE}_{10^{-}}$ rectangular to $\mathrm{TE}_{01}$-circular. Compressed output pulses are combined and absorbed in the load (2). This scheme is similar to SLED but for the addition of active elements (i.e., the resonance plasma switches). Employment of the Omega-P/NRL X-band magnicon [15] that is designed for a maximum output power of about $50 \mathrm{MW}$ in a 1 $\mu \mathrm{sec}$ pulse as a power source feeding the active pulse compressor described here allows one to anticipate a maximum output power of $500 \mathrm{MW}$ in the compressed 
pulse. Of course the resonance plasma switches (7) have to be replaced with the ferroelectric resonance switches for the tests referred to in this paper.

The main design parameters of the proposed active pulse compressor with ferroelectric switch are given in Table I

TABLE I. Parameters of the active pulse compressor.

\begin{tabular}{|l||l|}
\hline operating frequency $f_{0}$ & $11.424 \mathrm{GHz}$ \\
\hline input power $P_{o}$ & $50 \mathrm{MW}$ \\
\hline input pulse duration $t_{f}$ & $1 \mu \mathrm{sec}$ \\
\hline power gain $k$ & 10 \\
\hline peak output power $P_{\text {out }}=k P_{o}$ & $500 \mathrm{MW}$ \\
\hline output half-height pulse duration $t_{0.5}$ & $\sim 40 \mathrm{nsec}$ \\
\hline
\end{tabular}

\section{FERROELECTRIC SWITCH}

1. To determine the switch cavity parameters, it is necessary to calculate optimal coupling of the storage cavity in both operating regimes, namely in the energy storage regime and the energy extraction regime. All calculations have been carried out for the existing $\mathrm{ABC}$ storage cavities [14], even though superior performance could be anticipated from improved design of the cavities. The $\mathrm{TE}_{01}$ energy storage cavities have lengths of about $2 \mathrm{~m}$ including tapers and diameters of $8 \mathrm{~cm}$; their measured self quality factors $Q_{0}$ are 110,000 . Optimal coupling in the energy storage regime is determined by maximizing the filling efficiency. The efficiency $\eta$ for energy transfer to the storage cavity is [16]

$$
\eta=\frac{2 \beta \tau_{o}}{t_{f}(1+\beta)^{2}}\left(1-\exp \left(-\frac{t_{f}(1+\beta)}{\tau_{o}}\right)\right)^{2}
$$

where $\beta$ is the coupling, and $\tau_{o}=2 Q_{o} / \omega$ is the self time constant for the storage cavity. For parameters listed in Table I, the optimal coupling $\beta$ is 4.2 , which corresponds to a maximum efficiency of $64 \%$. Note that for the optimized cavity design the maximal achievable efficiency is about $82 \%$.

Adjustment of coupling in the energy extraction regime $\beta_{\text {out }}$ should allow one to achieve a desired power gain $k=P_{\text {out }} / P_{o}$, which can be calculated from the following expression for output power.

$$
P_{\text {out }}=\frac{\omega W_{\text {st }}}{Q_{o}} \beta_{\text {out }}=\frac{\omega W \eta}{Q_{o}} \beta_{\text {out }}=\frac{\omega P_{o} t_{f} \eta}{Q_{o}} \beta_{\text {out }}=2 P_{o} \frac{t_{f}}{\tau_{o}} \eta \beta_{\text {out }} .
$$

Now, the coupling in the energy extraction regime is found to be 


$$
\beta_{\text {out }}=\frac{1}{2 \eta} \frac{P_{\text {out }}}{P_{o}} \frac{\tau_{o}}{t_{f}}=\frac{k}{2 \eta} \frac{\tau_{o}}{t_{f}} .
$$

For power gain $k=10$, coupling $\beta_{\text {out }}$ in the energy extraction regime is 25 . The time constant $\tau_{\text {out }}$ in the energy extraction regime is $\tau_{\text {out }}=\tau_{0} /\left(1+\beta_{\text {out }}\right)=123 \mathrm{nsec}$, and the half-height pulse duration $t_{0.5}$ in this case is $43 \mathrm{nsec}$. The switch cavity is a two-port element inserted between the storage cavity and the mode converter; it is characterized by transmission coefficient $T$, that is related with the coupling $\beta$ in the following way:

$$
T^{2}=\beta \frac{4 L}{v_{g r} \tau_{0}},
$$

where $v_{g r}$ is the average group velocity in the storage cavity, $L$ is the storage cavity length. In the case considered ( $L=2 \mathrm{~m}$ and $\left.v_{g r} \sim \mathrm{c}\right), T^{2} \approx 9 \cdot 10^{-3} \beta$. Thus, the switch cavity transmission should be $-14 \mathrm{~dB}$ in the energy storage regime, and $-6.5 \mathrm{~dB}$ in the energy extraction regime.

2. As a result of simulation of the model switch cavity partially filled with ferroelectric (as shown in Fig. 3) two mode families were found. The first family, which are labeled "vacuum modes," are exemplified by tuning curves \#1 in Fig. 4; these have a small electric field in the ferroelectric, and thus their resonance frequencies don't depend sensitively on $\varepsilon$. The second family, which are labeled "ferroelectric modes," are exemplified by dashed curves \#2 in Fig.4; these have high electric field in the ferroelectric and thus their resonance frequencies exhibit a strong dependence of the frequency on $\varepsilon$. One can see that the resonance frequencies on curve $\# 2$ change by $\sim 10 \%$ when $\varepsilon$ changes by $20 \%$. Consequently, the ferroelectric mode could be controlled with weaker applied electric field than the vacuum mode.

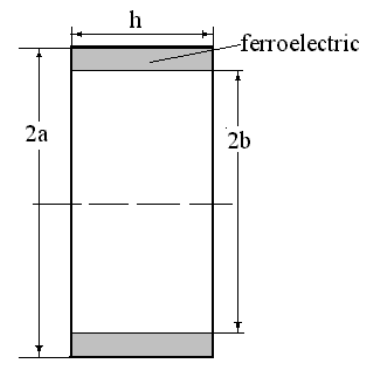

FIGURE 3. The model switch cavity schematic.

For the proposed $500 \mathrm{MW}, 11.424 \mathrm{GHz}$ active pulse compressor, where an available ferroelectric with $\varepsilon=500$ [13] is to be used, the resonance frequency of the switch cavity for the ferroelectric mode changes by $\sim 1 \mathrm{GHz}$ while $\varepsilon$ changes from 400 to 500 , but the resonance frequency for the vacuum mode changes by only $\sim 70 \mathrm{MHz}$. Unfortunately, the maximum electric field in the ferroelectric for the ferroelectric 
mode would be about $350-370 \mathrm{kV} / \mathrm{cm}$, which is much higher than the field for vacuum mode $(15-20 \mathrm{kV} / \mathrm{cm})$, and far above the breakdown limit of $\sim 100 \mathrm{kV} / \mathrm{cm}$. As a consequence, the vacuum mode is the only choice to be the operating mode for this type of active pulse compressor.

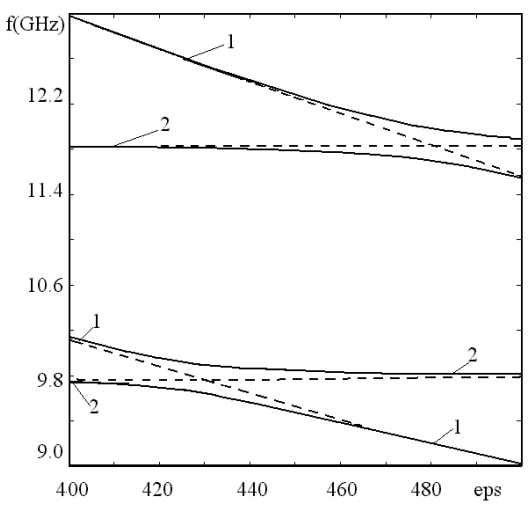

FIGURE 4. The model cavity spectrum $v s$. ferroelectric permittivity $\varepsilon$ for $\mathrm{a}=56 \mathrm{~mm}, \mathrm{~b}=53 \mathrm{~mm}$ and $\mathrm{h}=20 \mathrm{~mm}$.

3. The entire proposed ferroelectric switch arrangement is shown in Fig. 5, including the matching diaphragm and two $\mathrm{TE}_{031}$ switch cavities with ferroelectric rings. Two cavities are required because, in a switch containing only one cavity, the electric field in the ferroelectric is still too high for the design output power of $500 \mathrm{MW}$.

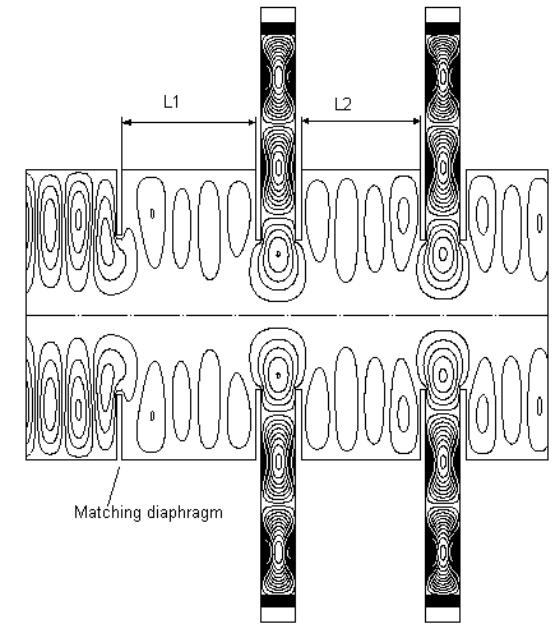

FIGURE 5. The switch conceptual layout. The diameter of the matching diaphragm is $30 \mathrm{~mm}$ and its thickness is $3 \mathrm{~mm} ; L_{1}=80 \mathrm{~mm}, L_{2}=78.5 \mathrm{~mm}$. 
Parameters found for the switch cavity are listed in the Table II.

TABLE II. Parameters of $\mathrm{TE}_{031}$ switch cavity.

\begin{tabular}{|l||l|}
\hline operating frequency, $\mathrm{GHz}$ & 11.424 \\
\hline operating mode & $\mathrm{TE}_{031}$ \\
\hline cavity length, $\mathrm{mm}$ & 20 \\
\hline cavity diameter, $\mathrm{mm}$ & 118 \\
\hline coupling iris diameter, $\mathrm{mm}$ & 30 \\
\hline coupling diaphragm thickness, mm & 3 \\
\hline ferroelectric ring length, mm & 20 \\
\hline ferroelectric ring inner diameter, mm & 106 \\
\hline ferroelectric ring thickness, mm & 3 \\
\hline transmission in energy extraction regime $(\varepsilon=400), \mathrm{dB}$ & 0 \\
\hline transmission in energy storage regime $(\varepsilon=500), \mathrm{dB}$ & -4 \\
\hline maximum electric field in ferroelectric in energy extraction regime, $\mathrm{kV} / \mathrm{cm}$ & 20 \\
\hline maximum electric field in ferroelectric in energy storage regime, $\mathrm{kV} / \mathrm{cm}$ & 10 \\
\hline maximum electric field in vacuum in energy extraction regime, $\mathrm{kV} / \mathrm{cm}$ & 865 \\
\hline maximum electric field in vacuum in energy storage regime, $\mathrm{kV} / \mathrm{cm}$ & 675 \\
\hline
\end{tabular}

The frequency dependences of transmission for the energy storage and energy extraction regimes for the entire system that includes the matching diaphragm and the two switch cavities are shown in Fig. 6.

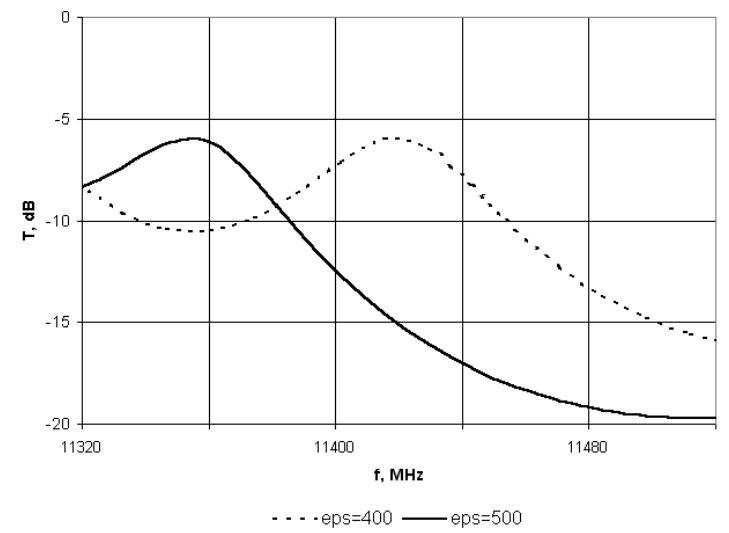

FIGURE 6. Transmission $T$ vs. frequency in the energy storage regime (solid curve), and the energy extraction regime (dashed curve). 
The solid curve corresponds to the energy storage regime, when both switch cavities have resonance frequencies of about $11.350 \mathrm{GHz}$, and transmission at the operating frequency is about $-14 \mathrm{~dB}$. In the energy extraction regime (dashed curve), the ferroelectric permittivity of the switch cavities is decreased from 500 to 400 , and the resonance frequencies are close to the operating frequency. At resonance, the self transmission of both cavities is close to $0 \mathrm{~dB}$, and the matching diaphragm provides the desired transmission of $-6.5 \mathrm{~dB}$ at $11.424 \mathrm{GHz}$.

4. A conceptual design of a switch cavity is shown in Fig. 7. The rf fields distribution for the operational mode $\mathrm{TE}_{0 \mathrm{mn}}$ allows one to separate part of the end wall from the rest of the cavity practically without distortion of the surface rf currents. This allows one to apply the bias voltage without additional electrodes (see Fig. 7). The biasing in this case is transverse with respect to rf electric field. It should be noted that, in BST ceramics, the change in $\varepsilon$ does not depend on the mutual orientation of bias and $\mathrm{rf}$ electric fields. Of course, the ends of ceramic ring must be metallized in order to prevent excessive electric fields between ceramic and the cavity walls. It is proven experimentally that copper is an acceptable material for metallization of BST ceramics [17].

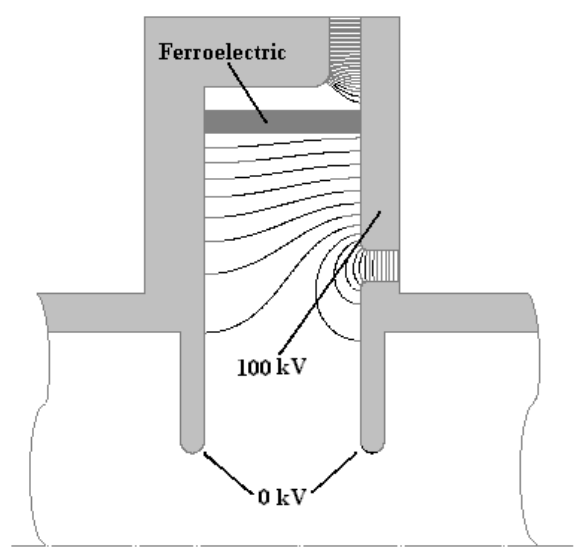

FIGURE 7. A concept design of a switch cavity with "transverse biasing", showing the bias electric field lines. Vacuum shell, high voltage bias feed through, etc. are not shown.

There are no fundamental size limitations for sintering of BST ceramic rings. Euclid Concepts, LLC has experience in the manufacturing of large diameter long tubes from ceramic powders analogous in composition and processing to BST ceramics. These tubes were used as dielectric waveguides in accelerating systems [18]. It should be noted that the ferroelectric element can be made as a set of small segments rather than as a cylinder. The gaps between ceramic segments will not materially affect rf field distribution in the switch cavity.

The switching time in the compressor is limited by the time needed to charge the capacitance of the ring and the surrounding structure up to the full voltage of $100 \mathrm{kV}$. 
This capacitance is in range $180-220 \mathrm{pF}$ for $\varepsilon=400-500$. So, in order to get a rise time of about $10 \mathrm{~ns}$, the biasing generator impedance should be about $25 \mathrm{Ohms}$.

\section{CONCLUSIONS}

A new ferroelectric switch has been designed as an element for a high-power $\mathrm{rf}$ pulse compressor at the NLC/JLC frequency of $11.4 \mathrm{GHz}$. Solutions found in the design allow one to anticipate output pulsed power of about $500 \mathrm{MW}$, which corresponds to the NLC/JLC requirements.

In addition to the main goal of achieving a compression ratio higher than that available with passive compressors, this new switch could also allow rapid variations in the coupling between an rf energy storage cavity, the load and the rf source during both the energy storage and energy release portions of the rf pulse. This attribute would lead to an increase in intrinsic efficiency of an active SLED well above $82 \%$, and an improved (i.e., flatter) shape of the output pulse.

\section{ACKNOWLEDGMENTS}

This work was supported by the Division of High Energy Physics, US Department of Energy.

\section{REFERENCES}

1. International Linear Collider Technical Review Committee, $2^{\mathrm{d}}$ Report, SLAC-R-606, SLAC2003

2. S.G. Tantawi, "New Development in RF Pulse Compression," SLAC-PUB-8582.

3. S.G. Tantawi, R.D. Ruth, A.E. Vlieks, and M. Zolotorev, "Active High-Power RF Pulse Compression Using Optically Switched Resonant Delay Lines," IEEE Trans. Microwave Theory Tech., v. 45, No 8, Aug 1997, p. 1486.

4. F. Tamura and S.G. Tantawi, "Development Of High-Power X-Band Semiconductor RF Switch For Pulse Compression Systems Of Future Linear Colliders," LINAC 2000, pp. 751-753.

5. A.L. Vikharev, A.M. Gorbachev, O.A. Ivanov, et al, "Active Microwave Pulse Compressor Employing Oversized Resonators and Distributed Plasma Switches," AIP Conf. Proc., 472, p. 975.

6. S.G. Tantawi and C.D. Nantista, "Active and Passive RF Components for High-Power Systems," SLAC-PUB-9499.

7. A.L. Vikharev, O.A. Ivanov, A.M. Gorbachev, V.A. Isaev, S.V. Kuzikov, S.H. Gold, A.K. Kinkead, O.A. Nezhevenko, J.L. Hirshfield, "High-Power Tests of a Two-Channel X-Band Active RF Pulse Compressor Using Plasma Switches," in present Proceedings.

8. S.G. Tantawi, "Overmoded High-Power RF Magnetic Switches and Circulators," Proc. PAC2001, Chicago, pp. 1216.

9. R.T. Farrar, "Spin-Lattice Relaxation Time in Yttrium Iron Garnet,” J. Appl. Phys., 1958, 29, p.425.

10. V.P. Yakovlev, O.A. Nezhevenko and J.L. Hirshfield, "Active Pulse Compressor with Ferroelectric Switch," PAC2003, Portland, May 11-16, 2003, TPAE031, to be published.

11.L.C. Sengupta, "Bulk Ceramic Ferroelectrics and Composites: Manufacture, Microwave Properties and Applications," IMS2000; see also http://my.ece.ucsb.edu/yorklab/Projects/Ferroelectrics/IMS2000\%20Workshop/WFE002.pdf

12. D. Webb, “Applications of Ferroelectrics in Military Systems," IMS2000; see also 
http://my.ece.ucsb.edu/yorklab/Projects/Ferroelectrics/IMS2000\%20Workshop/WFE004.pdf

13. E.A. Nenasheva, A.D. Kanareykin, N.F. Kartenko, and S.F. Karmanenko, "Ceramic materials based on $(\mathrm{Ba}, \mathrm{Sr}) \mathrm{TiO}_{3}$ solid solutions for tunable microwave devices," Int. Conf. on Electroceramics, MIT, Cambridge, MA, 2003, to be published; see also http://ice-2003.mit.edu/ice-oral2.pdf

14.A.L. Vikharev, A.M. Gorbachev, O.A. Ivanov, V.A. Isaev, V.A. Kondakov, S.V. Kuzikov, A.G. Litvak, M.I.Petelin, J.L. Hirshfield, and O.A. Nezhevenko, "Two-channel Active High-Power Xband Pulse Compressor," AIP Conf. Proc., v. 569, p. 741.

15.O.A. Nezhevenko, V.P. Yakovlev, J.L. Hirshfield, E.V. Kozyrev, S.H. Gold, A.W. Fliflet, and A.K. Kinkead, "Performance Of X-Band Pulsed Magnicon Amplifier," PAC2003, Portland, May 11-16, 2003, TPAE022, to be published.

16.Z.D. Farkas, et al, "SLED: A Method of Doubling SLAC's Energy," Proc. 9th Int. Conf. On High Energy Accelerators. SLAC, Stanford, CA, 1976, p.576.

17.A.I. Dedyk, S.F. Karmanenko, M.N. Malyshev, L.T. Ter-Martirosjan, "Investigation of dielectric hysteresis in multilayer structures on the basis of strontium titanate," Sol. State Phys. v.37(11), pp.3470-3477, 1995.

18.J.G. Power, M.E. Conde, W. Gai, A.Kanareykin, R. Konecny, and P. Schoessow, "First experimental measurements of wakefields in a multimode, dielectric structure driven by a train of electron bunches," Physical Review Special Topics of Accelerators and Beams, ST-AB, 3, 1013021-7, (2000). 


\section{AIP $\mid$ Proceedings}

\section{Fast XBand Phase Shifter}

V. P. Yakovlev, O. A. Nezhevenko, and J. L. Hirshfield

Citation: AIP Conference Proceedings 737, 643 (2004); doi: 10.1063/1.1842603

View online: http://dx.doi.org/10.1063/1.1842603

View Table of Contents: http://scitation.aip.org/content/aip/proceeding/aipcp/737?ver=pdfcov

Published by the AIP Publishing 


\title{
Fast X-Band Phase Shifter
}

\author{
V.P. Yakovlev ${ }^{1}$, O.A. Nezhevenko ${ }^{1}$, and J.L. Hirshfield ${ }^{1,2}$ \\ ${ }^{1}$ Omega-P, Inc., New Haven, CT 06511, USA, \\ ${ }^{2}$ Yale University, Physics Department, New Haven, CT 06520, USA
}

\begin{abstract}
A phase shifter to be the key element of an active high-power switch is described. This phase shifter employs ultra-fast, electrically-controlled ferroelectric elements. This high-power switch will allow one to build an active Delay Line Distribution System (DLDS), which would provide substantial reduction in the length of waveguide, compared to what would be required for the traditional passive DLDS design for NLC. The results of preliminary optimization of the phase shifter at the NLC frequency of $11.424 \mathrm{GHz}$ are presented showing the feasibility of building the switch to control a power of $500 \mathrm{MW}$. Initial tests at a power of up to $50 \mathrm{MW}$ are planned using the Omega-P/NRL X-band magnicon.
\end{abstract}

\section{INTRODUCTION}

The current design for the linear collider NLC relies on pulse compression to achieve the high peak RF power levels required to drive the accelerator structures ( $\sim 500-600 \mathrm{MW}$ in $400 \mathrm{~ns}$ pulses) [1]. A number of rf pulse compression systems have been considered recently for collider use, including versions of the Delay Line Distribution System (DLDS) and the Resonant Delay Line Pulse Compression System SLED-II [2]. The mechanisms upon which these systems operate are passive, in that no element in the microwave circuit has time-dependant properties. The RF pulse compression or power combining in all these systems is achieved using $180^{\circ}$ phase switching in the klystrons. Limitations of passive pulse compression systems include their low compression ratios ( 4:1), limited efficiencies, and very long runs of lowloss high-vacuum waveguide [2].

In principal, the most efficient power combining system should be DLDS. However, in its original incarnation, DLDS requires $\sim 300 \mathrm{~km}$ of waveguides and waveguide components [2]. It has been recognized that a substantial reduction in the length of waveguides could be achieved if an active DLDS system could be developed. Fig. 1 illustrates the layout of an active DLDS for the case of one waveguide feed for four accelerator modules [2]. The key element is a high power microwave switch, which must quickly divert $\sim 500 \mathrm{MW}$ of power sequentially from a main feed line in and out of the accelerator modules. The required peak power is achieved by adding coherently the outputs of eight 75 MW klystrons. A secondary attribute of active DLDS would be the absence of need for a pattern of fast phase shifting for the eight klystrons, thereby reducing the required klystron bandwidth. 


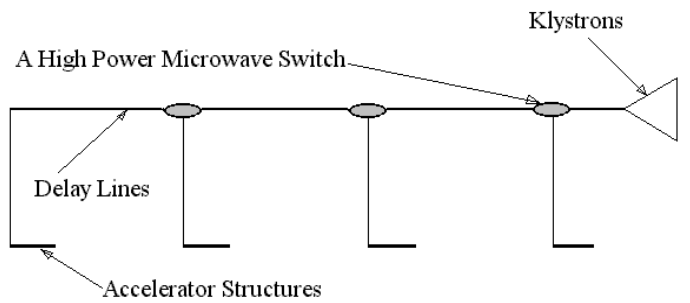

FIGURE 1. Schematic for an active DLDS that feeds four accelerator modules [2]. In this arrangement, the fast switches would each slice one-quarter of the length out of the $1.6 \mu$ s klystron pulse, and direct the $\sim 400$ ns slices to their respective accelerator structures so as to be synchronous with arrival of the beam.

In an attempt to circumvent limitations of passive pulse compressors and traditional DLDS, various concepts of high power switches have already received attention. These involve switches with optically-varied silicon mirrors [3], PIN/NIP diode arrays [4], and plasmas [5]. As yet, none of the tested versions of these active pulse compressors have achieved high enough power levels for use with NLC: the maximum output power achieved is $10 \mathrm{MW}$ in the switch using a PIN/NIP diode array active window [6], and $53 \mathrm{MW}$ in a compressor employing plasma switches [7].

In the present paper, a phase shifter to be the key element of an active high-power switch is described that employs ultra-fast, electrically-controlled ferroelectric elements $[8,9]$. Recently, ferroelectrics have received close attention, and are already used in low- to moderate-power (up to $100 \mathrm{~kW}$ peak) military and communication systems as fast tunable components $[10,11]$, because they have the ability to operate up to frequencies above $30 \mathrm{GHz}$ with reasonably low losses, and have very high tuning speed. These characteristics, together with the high electric breakdown strength and good vacuum properties, make modern ferroelectrics attractive candidates for use in high-power active RF phase shifters and switches for accelerator applications.

\section{GENERAL}

1. Ferroelectrics have an $\mathbf{E}$-field-dependent dielectric permittivity $\varepsilon(\mathbf{E})$ that can be rapidly altered by application of a bias voltage pulse. The switching time would be generally limited by the response time of the external electronic circuit [11] that generates and transmits the high-voltage pulse, and can thus be in the ns range. Ferroelectrics should have the following properties in order to function successfully in high-power rf switches for linear collider applications:

- the relative dielectric constant $\varepsilon$ should not exceed 300-500 to avoid problems in the switch design caused by interference from higher-order modes $[8,9]$;

- the relative dielectric constant $\varepsilon$ should change by 10 's of percent upon application of the bias voltage to provide the required tuning range; 
- bias electric fields should be of moderate amplitude, namely not more than a few 10 's of $\mathrm{kV} / \mathrm{cm}$, so as to avoid dielectric breakdown, and

- the loss tangent $\tan \delta$ should be about $10^{-3}$ or lower at $11 \mathrm{GHz}$. This is to insure that losses in the ferroelectric phase shifter will not exceed $1-2 \%$, thereby allowing switch operation at high average power in a collider having high repetition rate. For the losses of $1 \%$ at the NLC power of $500 \mathrm{MW}$ in the pulse of $1.6 \mu \mathrm{sec}$ and the repetition rate of $120 \mathrm{~Hz}$ the average dissipation in the phase shifter will be less than $500 \mathrm{~W}$.

Modern bulk ferroelectrics, such as barium strontium titanate $\left(\mathrm{Ba}_{\mathrm{x}} \mathrm{Sr}_{1-\mathrm{x}} \mathrm{TiO}_{3}\right.$, or BST) with $\varepsilon \sim 500$, have high enough electric breakdown strength $(100-200 \mathrm{kV} / \mathrm{cm})$ and do not require too high a bias electric field $(\sim 20-50 \mathrm{kV} / \mathrm{cm})$ to effect a significant change (20-30\%) in $\varepsilon$. Loss tangent for commercially-available samples of these materials is about $\sim 10^{-2}$ at $10 \mathrm{GHz}[10]$.

Euclid Concepts, LLC recently developed and tested a modified bulk ferroelectric based on a composition of BST ceramics, magnesium compounds, and rare-earth metal oxides that has a relative permittivity $\varepsilon=500$, and $20 \%$ change in permittivity for a bias electric field of $50 \mathrm{kV} / \mathrm{cm}$ [12]. In Fig. 2 is shown the first sample of a ring made of this material. This ring has outer diameter of $34 \mathrm{~mm}$, inner diameter of 20 $\mathrm{mm}$ and thickness of about $8 \mathrm{~mm}$. The ring dimensions are limited not by any fundamental questions, but merely by the presently-available fabrication equipment (specifically, the press-forms).

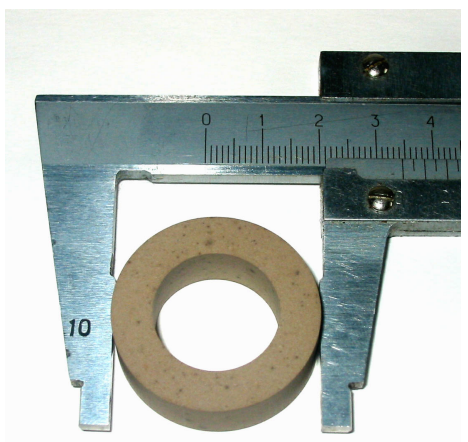

FIGURE 2. The first sample of a ring made of BST ceramic.

The loss tangent already achieved for the best samples is less than $4 \times 10^{-3}$ at $35 \mathrm{GHz}$ [12], which corresponds to about $1.3 \times 10^{-3}$ at $11 \mathrm{GHz}$, assuming the well-known linear dependence between loss tangent and frequency [10]. These losses are close to what is required for operation in NLC. Development of production techniques for this material continues, with the expectation of further lowering the loss tangent to values of less than $10^{-3}$. In principle, the availability of this ferroelectric already allows one to design and build an X-band high-power RF phase shifter rated for the full peak 
power of $500 \mathrm{MW}$, as required for NLC, but with a reduced repetition rate in order to avoid cooling problems.

2. Realization of an active DLDS (as shown in Fig. 1) requires development of a fast, high-power microwave switch. These switches must be able on command to redirect the full microwave power from a main feed line to one accelerator module after another within the RF pulse, with a switching time of tens of ns. One possible scheme for such a switch is shown in Fig. 3. The circuit consists of two 3-dB hybrid couplers (the passive elements), and an electrically-controlled phase shifter (the active element).

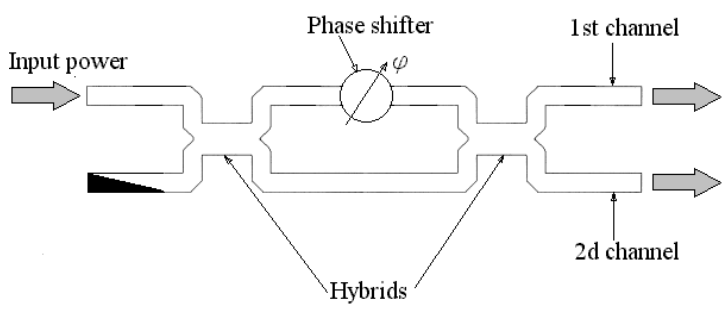

FIGURE 3. A possible arrangement of a switch for an active DLDS.

In this circuit, input power is supplied to one waveguide at the left, for instance from eight 75-MW klystrons, with the second left-most waveguide terminated with a matched load to absorb reflected power. The hybrid splits the input power into two equal portions which, with the phase shifter unbiased, combine into one of the rightmost output channels. In order to switch RF power from one output to the other, one has to change the phase difference between the two input signals by $180^{\circ}$. This change is to be provided by applying a fast rise-time bias voltage pulse across the ferroelectric elements in the high-power microwave phase shifter. This phase shifter has to be designed to operate with at least half of the full RF power $(500 / 2=250 \mathrm{MW}$ for NLC) in order to switch the full power of $500 \mathrm{MW}$ from the first channel to the second channel. For a 400 ns pulse to be directed to each accelerator section, the rise and fall times for the switching should each be less than about 30-40 ns, so as to not contribute a substantial loss of RF energy.

3. A phase shifter design can be constituted as a set of RF cavities partially-filled with ferroelectric whose dielectric constant is electrically-controlled. A change in resonance frequency of the cavities causes a corresponding phase change for the output signal.

The proposed ferroelectric phase shifter arrangement is shown in Fig. 4. The cavities should sustain the full power of $250 \mathrm{MW}$ without breakdown, and should possess low dielectric losses. These requirements limit the maximum available phase shift that can be achieved in one cavity to about $\sim 50^{\circ}$. Thus, in order to achieve the required phase shift of $180^{\circ}$, a sequence of four identical cavities should be used. The $\mathrm{TE}_{031}$ cavity mode is used in order to reduce the RF field in the ferroelectric, while keeping a reasonable diameter of the ferroelectric ring. The cavities have to be designed and positioned such a way that reflections of the input signal should be minimized and transmission should be maximized within a full tuning range of $180^{\circ}$. 
In order to achieve maximum transmission over a wide frequency range, a quarterwave filter-like arrangement may be used [13]. In this case the distance between the cavities should be close to $L=(n+1 / 2) \lambda_{z} / 2$. Here $n$ is an integer and $\lambda_{z}=2 \pi / k_{z}$, where $k_{z}$ is the longitudinal wavenumber in the waveguide sections between the cavities.

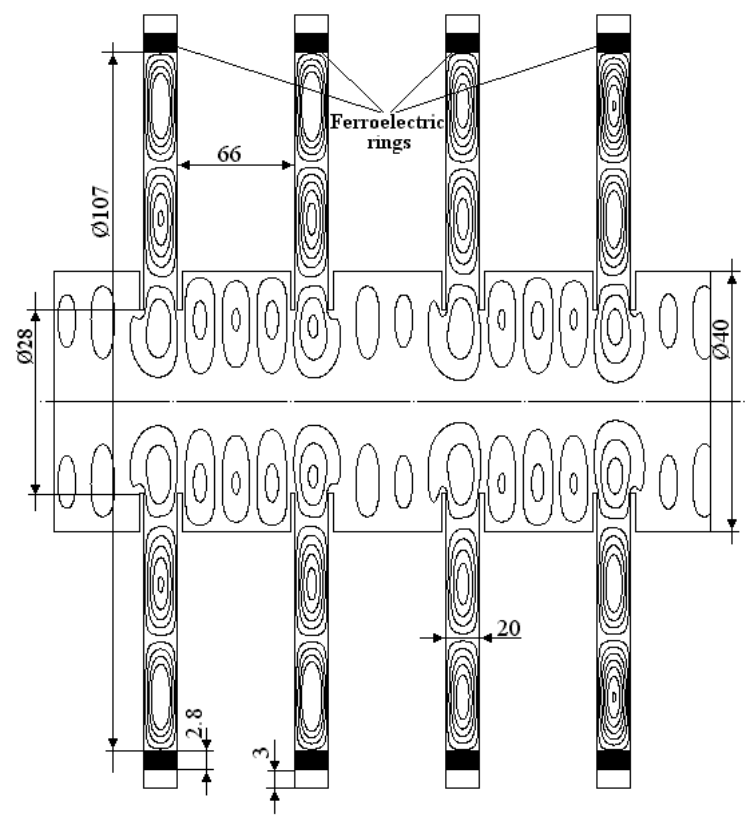

FIGURE 4. The entire phase shifter conceptual layout that includes four $\mathrm{TE}_{031}$ cavities, coupled together with sections of $\mathrm{TE}_{01}$ waveguide. All dimensions are in $\mathrm{mm}$.

The calculated change the phase of the transmitted signal vs. ferroelectric permittivity for the composite four-cavity phase shifter is shown in Fig. 5. One can see that this dependence is close to linear.

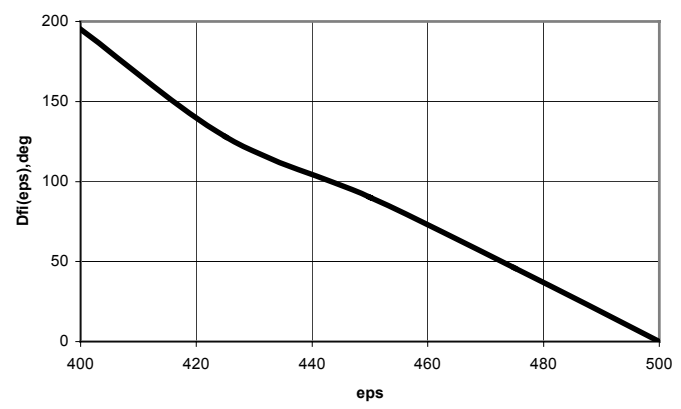

FIGURE 5. Phase change of the transmitted signal vs. $\varepsilon$. 

I.

The results for optimized parameters found for the phase shifter are listed in Table

TABLE I. Parameters of the phase shifter.

\begin{tabular}{|l|l|}
\hline operating frequency, $\mathrm{GHz}$ & 11.424 \\
\hline DC bias voltage for $\sim 200^{\circ}$ phase shift, $\mathrm{kV}$ & 100 \\
\hline number of cavities & 4 \\
\hline cavity operating mode & $\mathrm{TE}_{031}$ \\
\hline waveguide mode & $\mathrm{TE}_{01}$ \\
\hline maximum reflection within a full tuning range, $\mathrm{dB}$ & -27 \\
\hline transmitted (switched) power, MW & $250(500)$ \\
\hline peak power losses (tan $\delta=0.0013), \mathrm{MW}$ & $18(3.6 \%)$ \\
\hline efficiency, \% & $>96$ \\
\hline maximum RF electric field in ferroelectric, $\mathrm{kV} / \mathrm{cm}$ & 24 \\
\hline maximum RF electric field in vacuum, $\mathrm{kV} / \mathrm{cm}$ & 950 \\
\hline
\end{tabular}

It should be noted that further reduction of ferroelectric losses to increase the efficiency and lower the power to be dissipated is possible by improvement in the preparation of the ferroelectrics [12], by further optimizing the cavity design, and by increasing the number of cavities. All three means are planned for use in the full scale, high average power design for NLC.

4. A design concept for the phase shifter that will be suitable for high-power use is shown in Fig. 6.

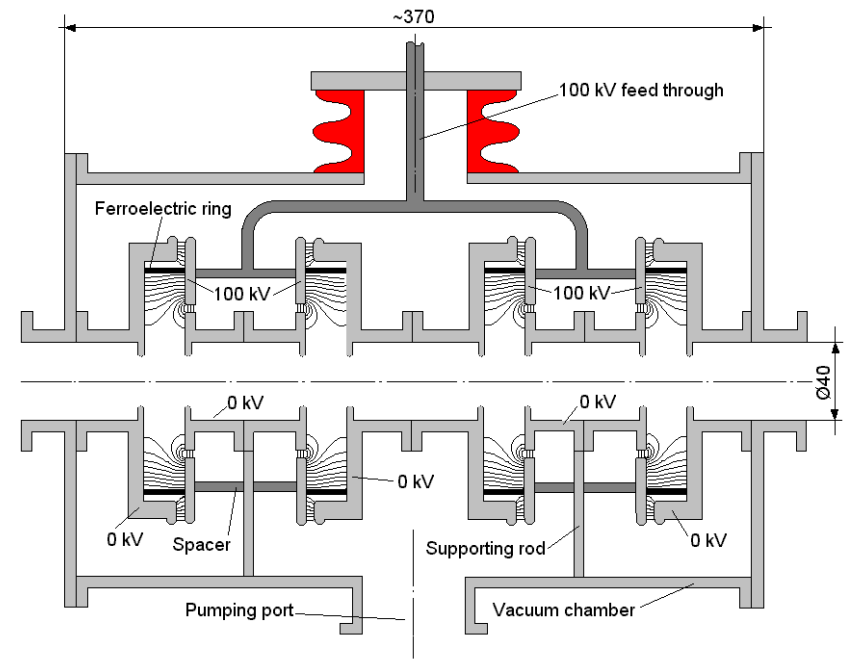

FIGURE 6. A concept of a phase shifter design, showing the bias electric field lines.

Dimensions are in $\mathrm{mm}$. 
The four cavities are located in an evacuated chamber that is external to the evacuated waveguide. In order to apply biasing voltages up to $100 \mathrm{kV}$, part of the end walls in each cavity are separated from the rest of the cavity by circular non-radiating slits. These wall segments are supported by special metallic spacer rods. These spacers are also used for applying biasing voltage from a single feed-through to all four cavities. The central parts of the cavities together with waveguides are supported by grounded supporting rods. Note that an additional small DC bias voltage (few of $\mathrm{kV}$ ) may be applied, if necessary, in order to suppress multipactoring near the ferroelectric rings and provide initial cavity tune.

The biasing voltage built-up time is limited by the capacitance of the ferroelectric rings. In our case the overall capacitance of all four rings is about $800 \mathrm{pF}$. Thus, in order to obtain a switching time of less than 40 ns required for linear collider application, the impedance of the biasing generator must be less than about 25 Ohms.

5. Evaluation of the phase shifter with RF power levels up to $50 \mathrm{MW}$ can be carried out using the Omega-P/NRL 11.4 GHz magnicon [14]. The proposed arrangement of the test bed for evaluation of the phase shifter using components developed for Omega-P active pulse compressor [5] is shown in Fig. 7.

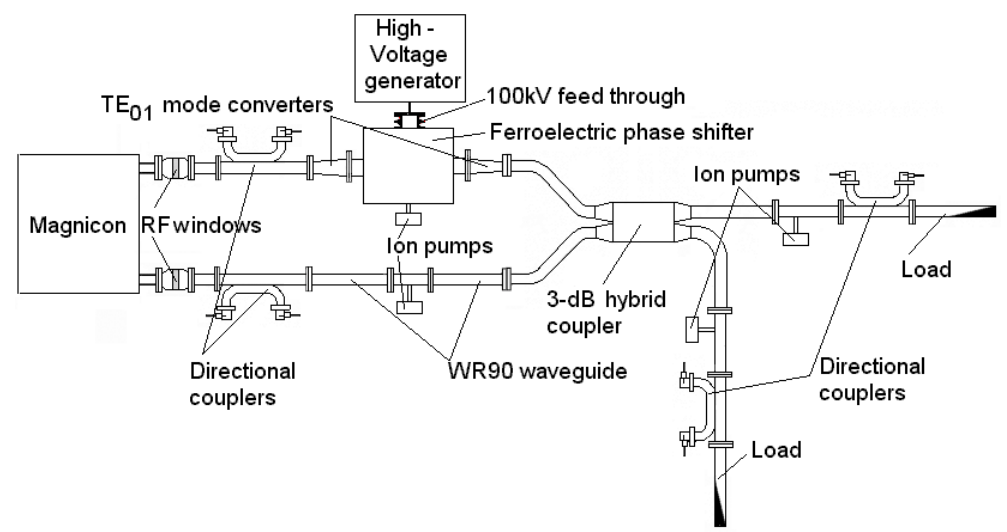

FIGURE 7. Arrangement for testing the phase shifter up to $50 \mathrm{MW}$.

Because the magnicon has two outputs with the equal power [14], the test bed for the phase shifter doesn't require a power splitter. Input and output of this phase shifter are circular waveguide with $\mathrm{TE}_{01}$ mode, and consequently the circuit requires two mode converters [5] from WR90 waveguide to circular waveguide. The second magnicon output is connected to a section of WR90 waveguide. The power from the two outputs is combined in a 3-dB hybrid [5]. Finally the power from the outputs of the two hybrids is absorbed in high-power SLAC-type vacuum loads. Varying the 100 $\mathrm{kV}$ voltage of the biasing pulse generator will allow redirection of the full magnicon power from one output arm of the 3-dB hybrid to another.

Tests at full power level can be performed at SLAC, the only laboratory where an $11.4 \mathrm{GHz}$ source with a power of $500 \mathrm{MW}$ in $400 \mathrm{nsec}$ pulses is presently available [15]. 


\section{CONCLUSIONS}

A new ultra-fast, electrically-controlled ferroelectric phase shifter has been designed as a key element of a high-power switch for an active DLDS for future linear collider. Solutions found in the design allow one to anticipate fast switching of Xband pulsed power at a level of about $500 \mathrm{MW}$, showing the possibility of developing an active DLDS for NLC.

\section{ACKNOWLEDGMENTS}

This work was supported by the Division of High Energy Physics, US Department of Energy.

\section{REFERENCES}

1. International Linear Collider Technical Review Committee, $2^{\text {nd }}$ Report, SLAC-R-606, SLAC 2003.

2. S.G. Tantawi, "New Development in RF Pulse Compression," SLAC-PUB-8582, Aug 2000.

3. S.G. Tantawi, R.D. Ruth, A.E. Vlieks, and M. Zolotorev, "Active High-Power RF Pulse Compression Using Optically Switched Resonant Delay Lines," IEEE Trans. On Microwave Theory and Tech., v. 45, No 8, Aug 1997, p. 1486.

4. F. Tamura and S.G. Tantawi, "Development Of High-Power X-Band Semiconductor RF Switch For Pulse Compression Systems Of Future Linear Colliders," LINAC 2000, pp. 751-753.

5. A.L. Vikharev, A.M. Gorbachev, O.A. Ivanov, et al, "Active Microwave Pulse Compressor Employing Oversized Resonators and Distributed Plasma Switches," AIP Conf. Proc., 472, p. 975.

6. S.G. Tantawi and C.D. Nantista, "Active and Passive RF Components for High-Power Systems," SLAC-PUB-9499, Sept 2002.

7. A.L. Vikharev, O.A. Ivanov, A.M. Gorbachev, V.A. Isaev, S.V. Kuzikov, S.H. Gold, A.K. Kinkead, O.A. Nezhevenko, J.L. Hirshfield, "High-Power Tests of a Two-Channel X-Band Active RF Pulse Compressor Using Plasma Switches,” RF2003, AIP Conf. Proc., 691, p. 197.

8. V.P. Yakovlev, O.A. Nezhevenko and J.L. Hirshfield, "Active Pulse Compressor with Ferroelectric Switch," PAC2003, Portland, May 11-16, 2003, pp.1150-1152.

9. V.P. Yakovlev, O.A. Nezhevenko, J.L. Hirshfield, and A.D. Kanareykin, "Ferroelectric Switch For An Active RF Pulse Compressor," RF2003, AIP Conf. Proc., 691, p. 187.

10. L.C. Sengupta, "Bulk Ceramic Ferroelectrics and Composites: Manufacture, Microwave Properties and Applications," IMS2000; see also http://my.ece.ucsb.edu/yorklab/Projects/Ferroelectrics/IMS2000\%20Workshop/WFE002.pdf

11. D. Webb, "Applications of Ferroelectrics in Military Systems," IMS2000; see also http://my.ece.ucsb.edu/yorklab/Projects/Ferroelectrics/IMS2000\%20Workshop/WFE004.pdf

12. A. Kanareykin, E. Nenasheva, A. Dedik, S. Karmanenko, V.P. Yakovlev, "New ferroelectric and ceramic materials intended for accelerator physics applications, " this Workshop.

13. J.L. Altman, "Microwave Circuits," D. Van Nostrand Company, Inc. Princeton, New Jersey, Toronto, New York, London, 1964.

14. A. Nezhevenko, V.P. Yakovlev, J.L. Hirshfield, E.V. Kozyrev, S.H. Gold, A.W. Fliflet, and A.K. Kinkead, "Performance Of X-Band Pulsed Magnicon Amplifier," PAC2003, Portland, May 11-16, 2003, pp.1128-1130.

15. S.G. Tantawi, C.D. Nantista, C. Adolphsen, E. Andrikopoulos, D. Burke, J. Chan, V. Dolgashev, K. Jobe, D. McCormick, J. Nelson, K. Racliffe, M. Ross, D. Schultz, T. Smith, D. Atkinson, Y.H. Chin, S. Kazakov, A. Lounine, T. Okugi, "Experimental Demonstration Of An RF System For The X-Band Linear Collider," SLAC-PUB-10550, July 2004. 


\title{
Fast ferroelectric phase shifters for energy recovery linacs
}

\author{
S. Yu Kazakov, ${ }^{1,2}$ S. V. Shchelkunov, ${ }^{3, *}$ V. P. Yakovlev, ${ }^{2}$ A. Kanareykin, ${ }^{4}$ E. Nenasheva, ${ }^{5}$ and J. L. Hirshfield ${ }^{1,3}$ \\ ${ }^{1}$ Omega-P, Inc., 258 Bradley Street, New Haven, Connecticut 06510, USA \\ ${ }^{2}$ Fermi National Accelerator Laboratory, Batavia, Illinois 60510, USA \\ ${ }^{3}$ Beam Physics Laboratory, Yale University, 272 Whitney Avenue, New Haven, Connecticut 06511, USA \\ ${ }^{4}$ Euclid Techlabs LLC, Solon, Ohio 44139, USA \\ ${ }^{5}$ Ceramics Ltd., St. Petersburg, 194223, Russia \\ (Received 18 May 2010; published 24 November 2010)
}

\begin{abstract}
Fast phase shifters are described that use a novel barium strontium titanate ceramic that can rapidly change its dielectric constant as an external bias voltage is changed. These phase shifters promise to reduce by $\sim 10$ times the power requirements for the rf source needed to drive an energy recovery linac (ERL). Such phase shifters will be coupled with superconducting radiofrequency cavities so as to tune them to compensate for phase instabilities, whether beam-driven or those caused by microphonics. The most promising design is presented, which was successfully cold tested and demonstrated a switching speed of $\sim 30 \mathrm{~ns}$ for $77 \mathrm{deg}$, corresponding to $<0.5 \mathrm{~ns}$ per deg of rf phase. Other crucial issues (losses, phase shift values, etc.) are discussed.
\end{abstract}

DOI: 10.1103/PhysRevSTAB.13.113501

PACS numbers: $84.40 .-\mathrm{x}, 41.90 .+\mathrm{e}, 29.20 .-\mathrm{c}$

\section{INTRODUCTION}

In energy recovery linacs (ERLs) there are several factors which significantly affect the required wall-plug power. With small beam loading, rf power requirements are determined by Ohmic wall losses, imbalance between beam currents, and microphonics. Compensation for the latter two typically requires a rapid change in coupling between the cavity and feeding line, and attendant high bandwidth, leading to a need for significant additional rf power. If beam loading is not small, there are beam-driven phase instabilities for which compensation will also demand additional power.

Compensation can be either by changing the cavity geometry to offset detuning caused by phase instabilities and/or microphonics [1,2], and/or to apply a corrective phase shift to the reflected rf wave that is reintroduced to the cavity so as to cancel phase instabilities $[3,4]$. The first strategy is accomplished by internal or external motors, or fast internal mechanical piezoelectric tuners. The second approach utilizes fast ferrite or ferroelectric phase shifters that are external to the cryomodules, whereas piezoelectric and other mechanical tuners require operation at cryogenic temperatures and thus permit only limited access in the event of a failure. Further, piezoelectric devices have mechanical resonances which may interfere with control system performance if their own resonance frequency overlaps with the microphonics excitation to be controlled [5]. It has been reported that the piezoelectric tuners may operate as fast as $\sim 25 \mathrm{kHz}$ ([see, e.g., Ref. [6], slide 11); however, it is still unknown if piezoelectric tuners are efficient enough at high frequencies.

\footnotetext{
*sergey.shchelkunov@gmail.com
}

Ferrite phase shifters [7-9] are presently limited in their response time to $\sim 30 \mu \mathrm{s}$, while the required response time may be only a few $\mu$ s. The limitation comes mainly from the eddy currents in the ferrite material [8].

The need for $\mu$ s response time is dictated by the phase and amplitude stability requirements of $\sim 0.06 \mathrm{deg}$ and $3 \times 10^{-4}$, as cited for the Cornell ERL [10]; requirements are similar for the electron cooler project at BNL [11]. The gain in the control feedback loop should be high enough, and its bandwidth wide enough, to ensure this high degree of stability. This translates to a bandwidth of about $1 \mathrm{MHz}$, and rules out contemporary ferrite tuners.

The authors have studied several designs for a fast electrically controlled ferroelectric phase shifter for ERL applications. The device is to allow changing the rf coupling during the cavity filling process in order to effect significant power savings, and also to provide rapid compensation for beam imbalance and allow for fast stabilization against phase fluctuations caused by microphonics and beam-driven instabilities. This capability should allow a reduction by about an order of magnitude in the required power from the rf source.

\section{POSSIBLE RF-POWER SAVINGS}

The rf power $P_{g}$ required to maintain an accelerating voltage $V$ is given by [12]

$$
\begin{aligned}
P_{g}= & \frac{V^{2}(1+\beta)^{2}}{4 \beta Q_{0}(r / Q)}\left\{\left(1+\frac{I_{\mathrm{Re}}(r / Q) Q_{0}}{V(1+\beta)}\right)^{2}\right. \\
& \left.+\left[\frac{Q_{0}}{1+\beta}\left(\frac{\omega_{0}}{\omega}-\frac{\omega}{\omega_{0}}\right)-\frac{I_{\mathrm{Im}}(r / Q) Q_{0}}{V(1+\beta)}\right]^{2}\right\},
\end{aligned}
$$

where $\omega_{0}$ is the cavity resonance frequency; $Q_{0}$ is its unloaded quality factor; $\beta$ is the coupling factor, for SC 
cavity $\beta \gg 1 ; r / Q$ is the cavity impedance; $I_{\mathrm{Re}}=$ $I\left(\cos \delta \phi_{a}-\cos \delta \phi_{d}\right), \quad I_{\mathrm{Im}}=I\left(\sin \delta \phi_{a}-\sin \delta \phi_{d}\right), \quad \delta \phi_{a}$ and $\delta \phi_{d}$, are the average phases of the accelerating and decelerating beams compared with the rf phase; and $I$ is the beam current. The value $\delta \omega=\omega_{0}-\omega$ is determined by the amplitude of uncontrolled noise.

In $[11,13]$, an example is given for a cooler linac having two cavities with $Q_{0} \approx 4.5 \times 10^{10}$ at $2 \mathrm{~K}$ and $r / Q \approx$ $400 \mathrm{Ohms} /$ cavity, $I=50 \mathrm{~mA} \times 2=100 \mathrm{~mA}$ and $V \approx$ $25 \mathrm{MV}$. (The beams go through the linac twice.) The intrinsic rf power required for the parameters listed above,

$$
P_{\mathrm{int}}=\frac{V^{2}}{Q_{0}(r / Q)}=9 \mathrm{~W}
$$

If the accelerated and decelerated beams are well balanced, and the beams are in phase with the rf field, the required power is determined by the peak frequency variations caused by microphonics [2], namely,

$$
P_{g}=\frac{V^{2}}{4 Q_{l}(r / Q)}\left[1+\left(2 Q_{l} \frac{\delta \omega}{\omega}\right)^{2}\right]
$$

where $Q_{l}$ is loaded quality factor, $Q_{l}=Q_{0} /(1+\beta)$. One finds the optimal value of the loaded quality factor, $Q_{\mathrm{opt}}=$ $\omega / 2 \delta \omega$, and the minimum required power is proportional the peak cavity detuning, namely,

$$
P_{g}=\frac{V^{2}}{(r / Q)} \frac{\delta \omega}{\omega}=0.55 \mathrm{~kW} \times d f[\mathrm{~Hz}]
$$

where $\delta f=\delta \omega / 2 \pi$ is the peak microphonic cavity detuning in Hz. If, for example, the peak cavity detuning is reduced to $30 \mathrm{~Hz}$ (a typical value), the required input power would be $\sim 17 \mathrm{~kW}$ for four five-cell cavities.

While beam loss within reasonable limits gives no significant increase in required power, the phase error $\delta \phi$ of the beams does, because in this case the beam introduces an additional reactance proportional to $\delta \phi$, as can be seen from (1). The required power in this case is

$$
\begin{aligned}
P_{g} & =\frac{V^{2}}{(r / Q)}\left(\frac{\delta \omega}{\omega}+\frac{(r / Q) I \delta \varphi}{2 V}\right) \\
& =0.55 \mathrm{~kW} \times d f[\mathrm{~Hz}]+22 \mathrm{~kW} \times \delta \varphi\left[^{\circ}\right] .
\end{aligned}
$$

With, for example, $\delta \phi=1^{\circ}$ and $\delta f=30 \mathrm{~Hz}$, the required power would be about $40 \mathrm{~kW}$. Obviously, it is crucial to provide a means for compensation of phase instabilities in the cited example, as well as in any other ERL [see, e.g., [14], slides 42-44] to keep the rf-power requirements to a minimum.

The most popular scheme [7-9] among several others to utilize phase shifters is shown in Fig. 1

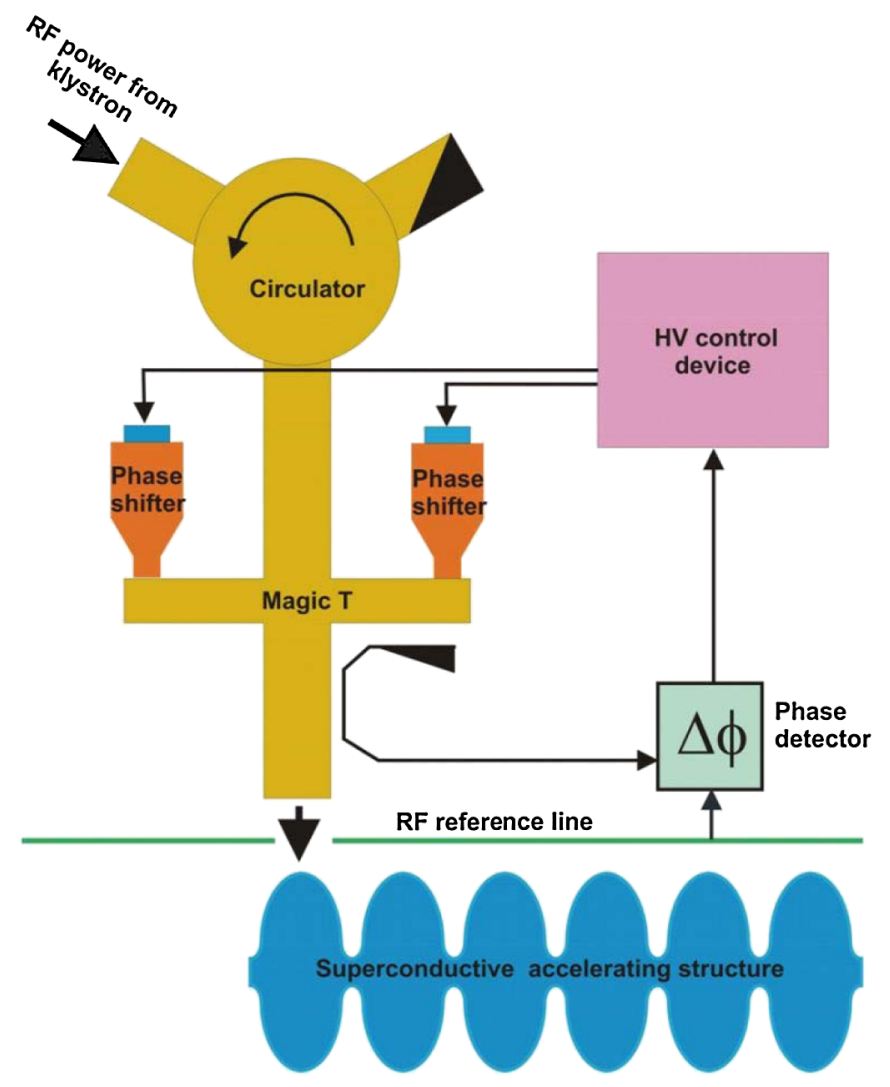

FIG. 1. External tuner in a magic- $T$ configuration with adjustable phase shifters. Each phase shifter works in one-port configuration, with its second port being shortened.

\section{FERROELECTRIC MATERIAL AS BASIS OF THE PROPOSED DEVICE}

Recently, ferroelectric (FE) devices for fast switching applications have received close attention, and are already used up to $100 \mathrm{~kW}$ peak in military systems [15], phasedarray radars [16], and communication systems [17]. FE's have a dielectric permittivity $\varepsilon(\mathbf{E})$ that depends on electric field $\mathbf{E}$, and can be rapidly altered by application of an external bias-voltage pulse. The response time would be limited by that of the external bias circuit. The minimum intrinsic switching time demonstrated is less than $1 \mathrm{~ns}$ [17]. Modern bulk ferroelectrics, e.g. $\mathrm{Ba}_{x} \mathrm{Sr}_{1-x} \mathrm{TiO}_{3}$ (barium strontium titanate or BST) with $\varepsilon \sim 500$, have sufficiently high electric-breakdown strength $(100-200 \mathrm{kV} / \mathrm{cm})$ and require an acceptable bias electric field $(\sim 20-50 \mathrm{kV} / \mathrm{cm})$ to effect a $20 \%-30 \%$ change in $\varepsilon$. Loss tangent for commercially available samples is about $\sim 1.5 \times 10^{-3}$ at $1 \mathrm{GHz}$ [16].

Euclid Techlabs LLC recently developed and tested a modified bulk FE [18] based on a composition of BST ceramics, magnesium compounds, and rare-earth metal oxides. The availability of this FE already allows one to create a high-power rf phase shifter with the peak power required for ERL. 
TABLE I. Properties of modified BST ferroelectric ceramic.

\begin{tabular}{|c|c|}
\hline Dielectric constant, $\varepsilon$ & $\sim 460$ \\
\hline Tunability, $\partial \varepsilon / \partial E_{\text {bias }}$ & $>2 /(\mathrm{kV} / \mathrm{cm})$ \\
\hline Intrinsic response time & $<10 \mathrm{~ns}$ \\
\hline Loss tangent at $1.3-1.4 \mathrm{GHz}, \tan (\delta)$ & $2 \times 10^{-3}$ \\
\hline Loss tangent at $700-900 \mathrm{MHz}, \tan (\delta)$ & $1.1 \times 10^{-3}$ \\
\hline Breakdown limit & $200 \mathrm{kV} / \mathrm{cm}$ \\
\hline Thermal conductivity, $K$ & $7.02 \mathrm{~W} / \mathrm{m} \mathrm{K}$ \\
\hline Specific heat, $C$ & $0.605 \mathrm{~kJ} / \mathrm{kg} \mathrm{K}$ \\
\hline Density, $\rho$ & $4.86 \mathrm{~g} / \mathrm{cm}^{3}$ \\
\hline Coefficient of thermal expansion & $10.1 \times 10^{-6} \mathrm{~K}^{-1}$ \\
\hline Temperature tolerance, $\partial \varepsilon / \partial T$ & $3 \mathrm{~K}^{-1}$ \\
\hline
\end{tabular}

Properties of modified BST FE ceramic are in Table I.

For the proposed devices, the FE ceramic is manufactured in the form of rings [Fig. 2(a)] or bars [Fig. 2(b)]. To measure the loss tangent for ringlike samples, the setup

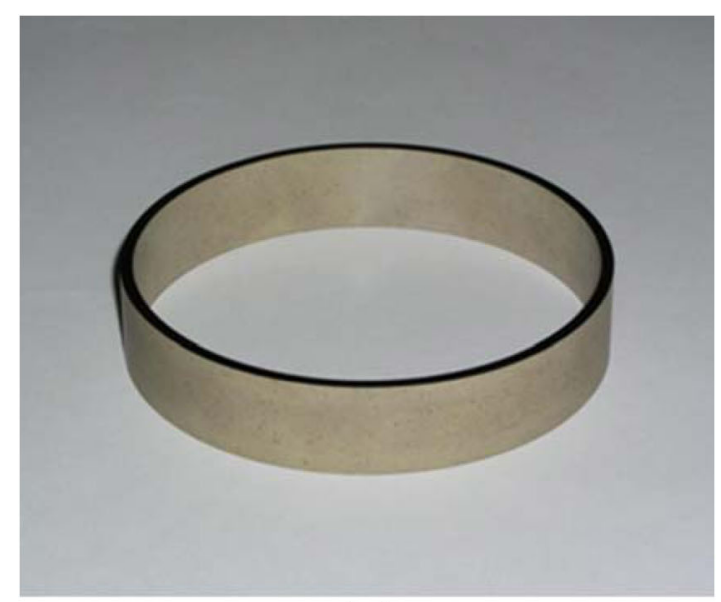

(a)

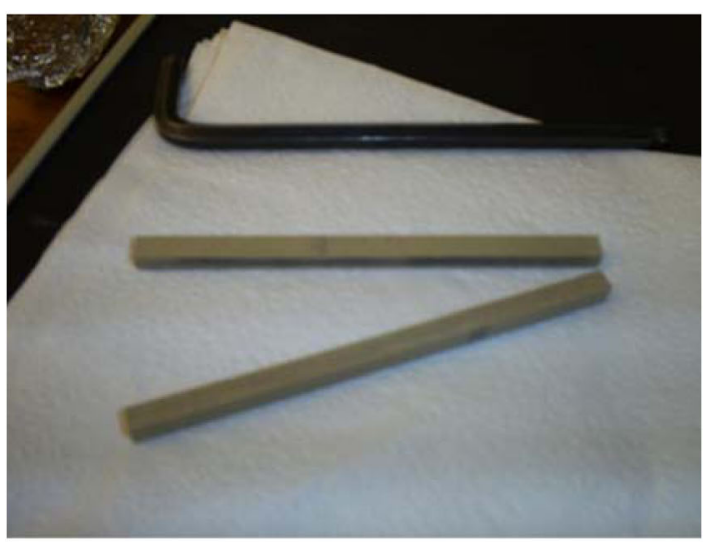

(b)

FIG. 2. (a) FE ceramic ring $\varnothing 106 \times 2.8 \times 22 \mathrm{~mm}$. Typically the top and bottom of the ring are fully coated with a thin, goldbased metallization layer (in a process proprietary to Ceramic, Ltd. (St. Petersburg, 194223, Russia). (b) FE ceramic bars $(6 \times 5 \times 108 \mathrm{~mm})$. shown in Fig. 3 was used [19]. Measurements on the bars were done with the bars suspended along the axis in a long metal pipe. In both cases, the values of the loss tangent were inferred in a manner as described in the caption to Fig. 3

Figure 4 presents results of measurements of tunability [changes (or relative changes) in the dielectric constant when an external electric field or voltage is applied]. To measure the tunability, one for instance uses a setup similar to that in Fig. 3, and applies a bias to upper and lower halves of the cavity thereby creating an electric field across the ring(s). The changes in the mode spectrum are observed, and the frequencies with and without the applied electric field are compared. Again, using e.g. HFSS, one forces the simulated modes' frequencies to undergo the same change

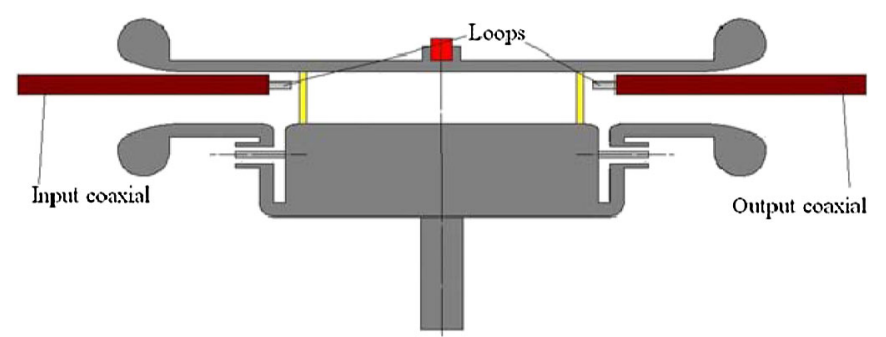

FIG. 3. An example of setup to measure FE ring loss tangent. Because of a large dielectric constant of the ceramic, modes exist with their fields almost entirely contained in the ceramic. In measurements, one may excite these modes using loops, measure their $Q$ factors, and then in, e.g., HFSS simulations force the simulated $Q$ factors be as the observed ones by adjusting the loss tangent of the ceramic in the simulation model which reproduces the real configuration. Hence, the value of the loss tangent is inferred for a set of frequencies corresponding to the mode spectrum.
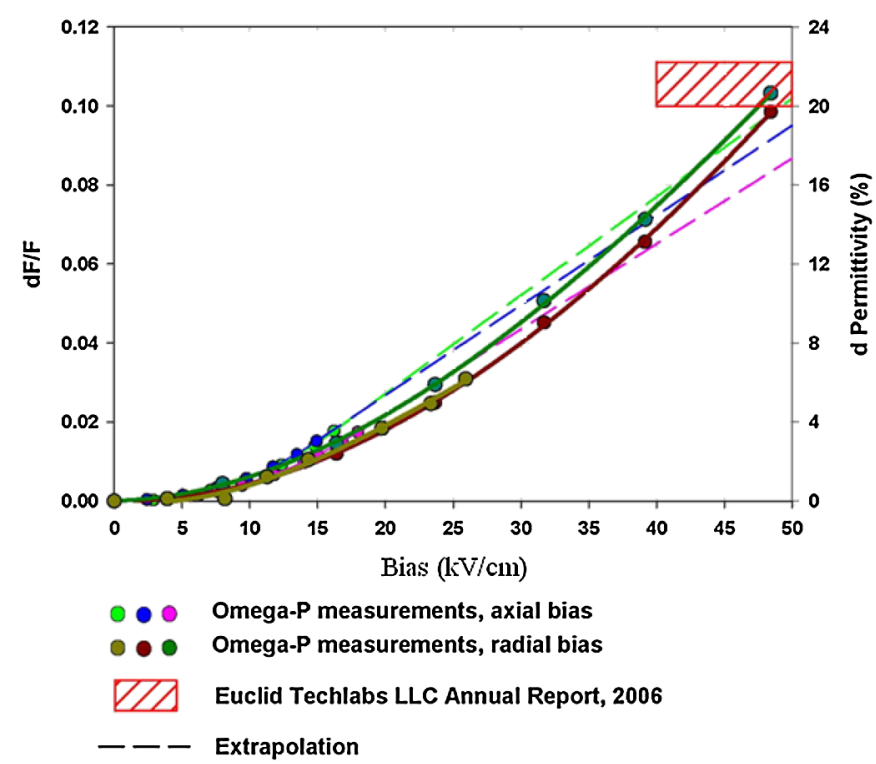

FIG. 4. Tunability measurements on modified BST ring. Note that one division on the right vertical axis is $4 \%$. 
by adjusting the dielectric constant of the ferroelectric material in the simulation model that reproduces the real configuration. Hence, the change in dielectric constant (and consequently, the tunability) can be inferred.

In Fig. 4, the lower portion of the curve indicates low tunability at lower applied voltages. Presently, efforts are underway to reduce the loss tangent at $1.3 \mathrm{GHz}$, without undue sacrifice of tunability.

\section{PHASE SHIFTER DESIGN}

Three configurations have been considered: coaxial, planar/coaxial hybrid, and sandwich-in-waveguide; all for $500 \mathrm{~kW}$ pulse and $4-5 \mathrm{~kW}$ average powers, figures dictated by ILC parameters that we have chosen as the base line. Below we describe the last of these which was successfully built, and cold tested.

The sandwich-in-waveguide configuration employs a standard WR650 waveguide as a host for three sets of two narrow ferroelectric (FE) bars and two matching ceramic slabs $(\varepsilon \sim 21)$, as shown in Fig. 5(a). Each set rests on a metal plate, with a second metal plate above, as seen in Fig. 5(b). The sides of the FE bars and matching slabs that are in contact with the metal electrodes are metalized with gold-based coating layers in a process that is proprietary to Ceramics, Ltd. (St. Petersburg, 194223, Russia).

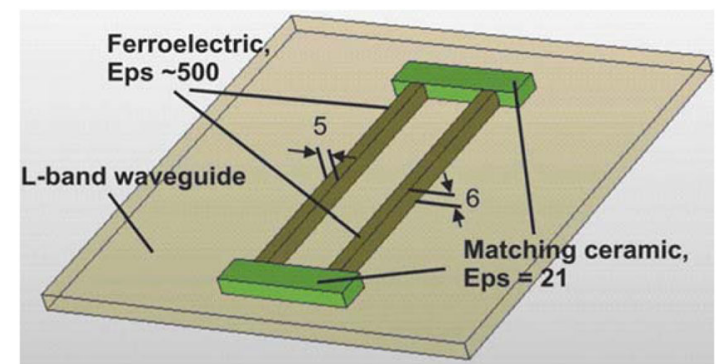

(a)

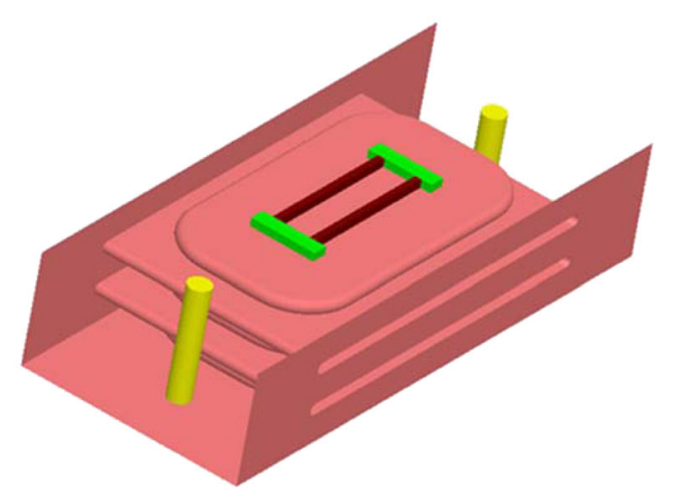

(b)

FIG. 5. (a) Arrangement for one set of FE bars (dark green) and ceramic slabs (light green); dimensions are in $\mathrm{mm}$. (b) WR650 waveguide with top removed to show three "sandwiches" and matching rods.

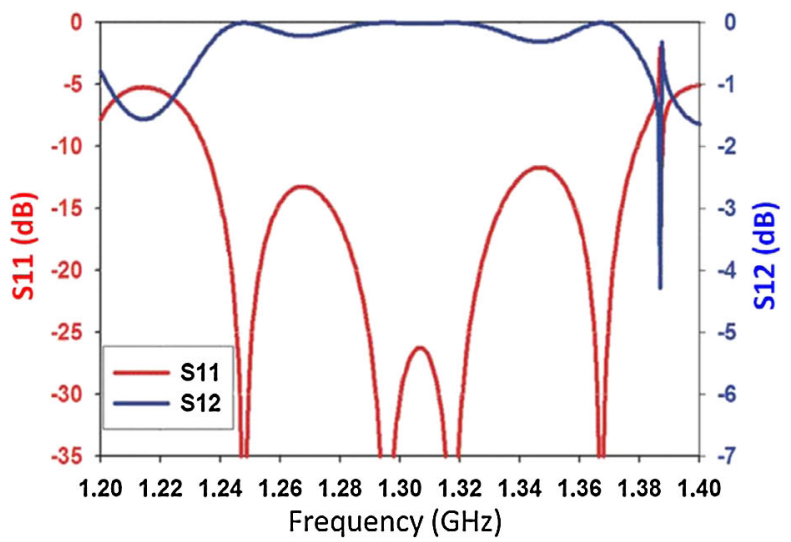

(a)

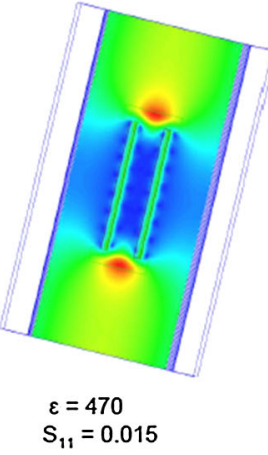

$S_{11}=0.015$

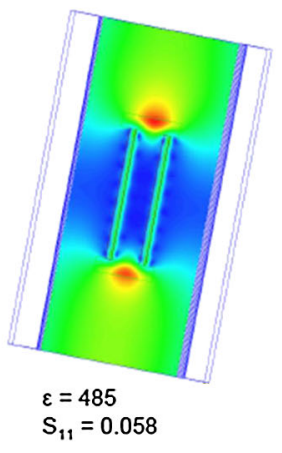

(b)

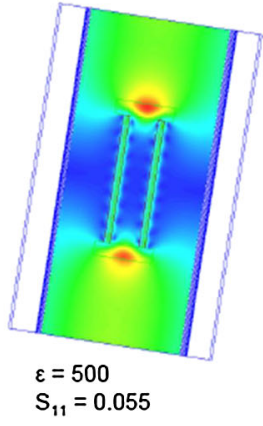

$\varepsilon=500$
FIG. 6. (a) Frequency response for sandwich geometry with $\varepsilon \sim 470$; it is nearly the same for $470<\varepsilon<500$. (b) Field pattern demonstrates that the geometry is well matched for different values of the dielectric constant of the ferroelectric.

Alternate plates are joined to an electric feedthrough to provide the desired bias, while other plates are grounded. When assembled, dimensions are $8.2 \mathrm{~cm} \times 16.5 \mathrm{~cm} \times$ $30 \mathrm{~cm}$. The mode spectrum is sparse, and can be controlled by changing the geometry. For matching to the structure, dielectric rods (alumina with $\varepsilon \sim 9.8$ ) are placed before and after the sandwiches. Frequency response is shown in Fig. 6(a).

Table II lists design parameters of the phase shifter for $500 \mathrm{~kW}$ of pulsed and $4 \mathrm{~kW}$ of average power.

\section{LOSS, PHASE SHIFT, AND SWITCHING SPEED MEASUREMENTS}

Low-power rf measurements were made using only one of the three sandwiches in a waveguide that has the same width as WR650, but tapered to one-third the standard height, as shown in Figs. 7(a) and 7(b). The central electrode can be biased electrically.

The loss tangent of ferroelectric bars is measured for the uncoated bars (manufactured from the same batch used to make the bars coated with gold-based metallization layers, as used in the $1 / 3$ model). The value of loss tangent is determined to be $\sim 2 \times 10^{-3}$, suggesting that the $1 / 3$ 
TABLE II. Design parameters of the phase shifter.

\begin{tabular}{lc}
\hline \hline FE permittivity $\varepsilon$ at $V_{\text {bias }}=0$, & 460 \\
$\partial$ (phase) $/ \partial \varepsilon$, deg & 4 \\
Maximum DC electric field, $\mathrm{kV} / \mathrm{cm}$ giving $\Delta$ (phase) $=120$ deg & 15 \\
Total loss, $\%$ & $2.8+6 \times 10^{3} \tan (\delta)$ \\
Maximum $E$ field in FE, $\mathrm{kV} / \mathrm{cm}$ & 3 \\
Maximum $E$ field in ceramic, $\mathrm{kV} / \mathrm{cm}$ & 5.9 \\
Maximum $E$ field in air, $\mathrm{kV} / \mathrm{cm}$ & 6.1 \\
Phase shift, deg, at $15 \mathrm{kV} / \mathrm{cm}$ bias & 120 \\
FE pulse heating with loss tan $5 \times 10^{-4}$ & $0.2 \mathrm{~K}$ for $\Delta \varepsilon=0.6$ \\
FE average heating with loss tan $5 \times 10^{-4}$ & $0.9 \mathrm{~K}$ for $\Delta \varepsilon=2.7$ \\
FE pulse heating with loss tan $2 \times 10^{-3}$ & $\sim 0.4 \mathrm{~K}$ for $\Delta \varepsilon=0.6$ \\
FE average heating with loss tan $2 \times 10^{-3}$ & $\sim 3.5 \mathrm{~K}$ for $\Delta \varepsilon=2.7$ \\
\hline \hline
\end{tabular}

scaled tuner model may suffer a transmission loss no better than $\sim 0.7 \mathrm{~dB}$. In actuality, the measured transmission is worse. The best value obtains only when one uses either freshly applied liquid indium-gallium or solders the bars to the waveguide walls using indium. However, we were not able to apply more than $4 \mathrm{kV}$ to the soldered configurations; hence, we discuss below only the structures assembled with liquid indium-gallium. It is found that in the transmission drops when the voltage increases (see Fig. 8).

It is highly questionable, but still anticipated, that a development of coating layers, which do not degrade during brazing, and successful brazing of the ferroelectric and matching dielectric bars will eliminate losses beyond those in the bulk ceramics and metallic walls, as well as to lead to transmission being independent of applied voltage. As of now, several brazing attempts have revealed that the gold coating of the bar surfaces suffers badly when subjected to rapidly rising temperature and, in addition, the brazing atmosphere must be thoroughly controlled to avoid traces of oxygen.
Results of measurements of phase shift are presented in Fig. 9. Again, tests were made with gold-plated ferroelectric bars and matching slabs and the contact to copper walls was provided by liquid indium-gallium alloy. The obtained values of the phase shift are seen to be in good agreement with expectations. However, it seems that the coated ferroelectric samples suffer some sort of a "shock" when exposed to high voltages; as the result, the observed dielectric constant would not return to its original value once the voltage is completely removed. It is highly questionable if this effect is of hysteresis-like nature, that is, if the dielectric constant will return to its original value when the FE ceramic is biased with some voltage of the opposite sign. The shock nature of this effect suggests that some residual shift in the dielectric constant is going to either remain and only disappear with passing time, or even grow further when an increased bias (of the same or opposite sign) is being continuously applied. Further research is planned to address this issue; in particular, one should understand if the observed effects are due to the particular composition of the ceramic itself, or because of complex

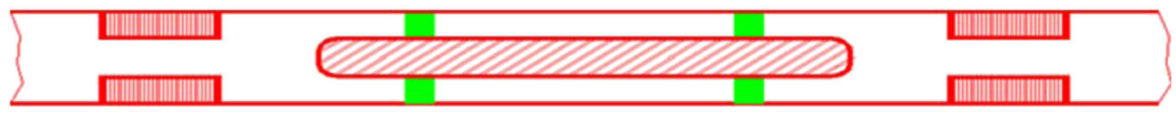

(a)

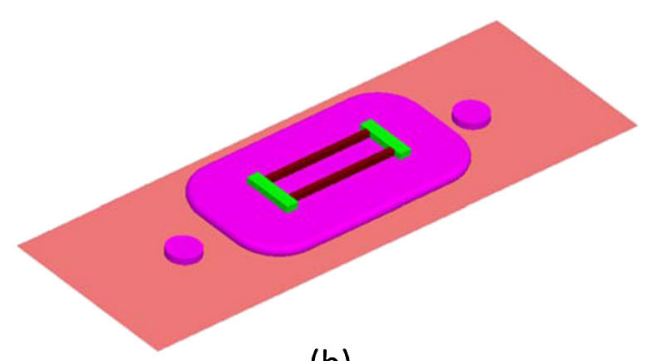

(b)

FIG. 7. (a) A cross-sectional side view of the one-third structure used for tests. Four rectangular boxes (two on the left, and two on the right) represent matching metal insertions; four square boxes (green) located above and below the electrode in the middle represent matching ceramic slabs. Ferroelectric rods are not seen in this cross section. (b) 3D view of the device used in the described below tests; the top wall (with two matching metal "tablets") and side walls are removed for clarity. 


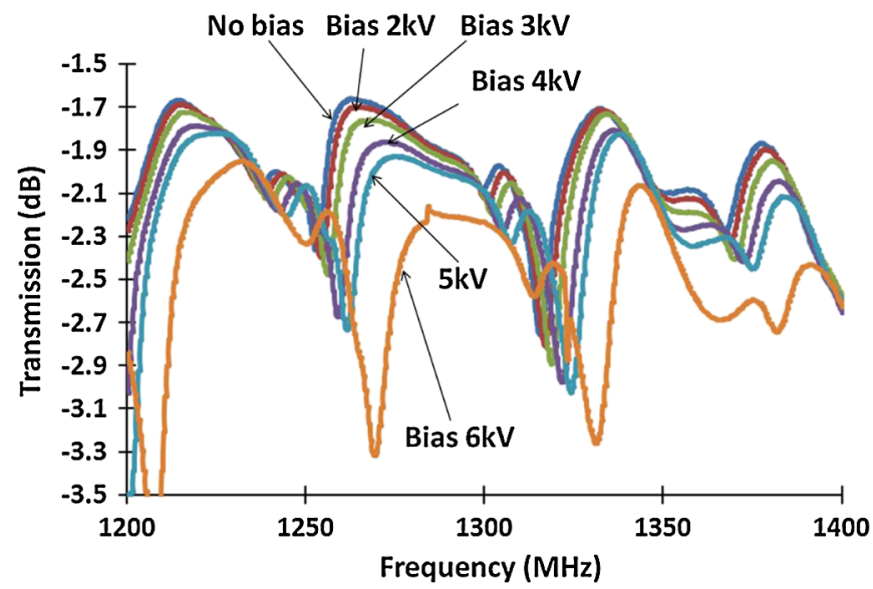

FIG. 8. The transmission drops when the voltage grows (see the magnitude change at $1.3 \mathrm{GHz}$ ).

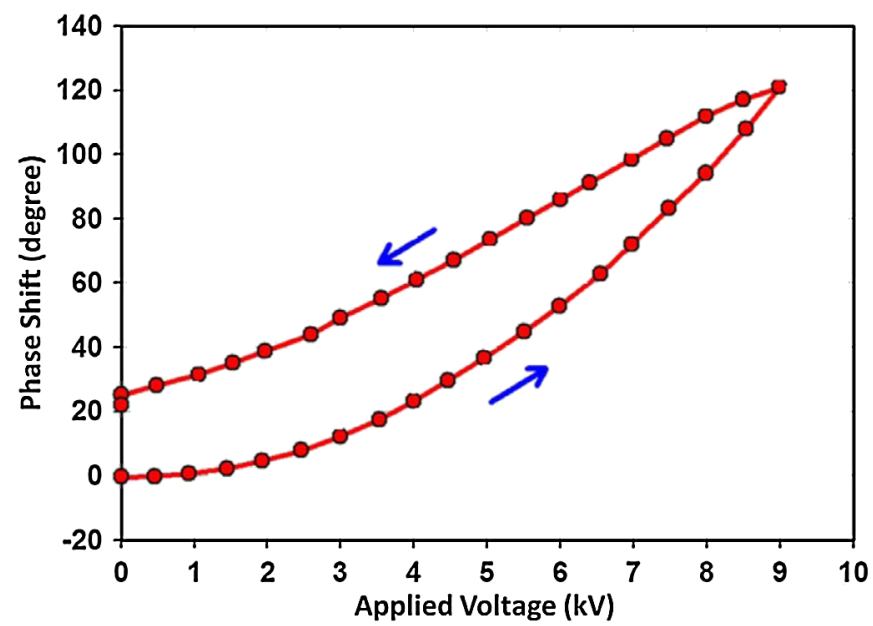

FIG. 9. Measured phase shift of rf signal transmitted through one-third section vs applied bias voltage.

physics happening in the transition layer between the ceramic and its metallization.

A vital property of any tuner is its response time, which for many accelerator applications should be less than 100 ns. Measurements of response time were made using the arrangement shown in Fig. 10. The high-voltage rise/ fall times from the available pulse generator were in the range of $\sim 100 \mathrm{~ns}$ (measured as the time difference from $5 \%$ to $95 \%$ of the voltage maximum). Switching speed measurements (each averaged over 16 shots) were processed by subtracting data with rf off from data taken with rf on, and are shown in Fig. 11.

The difference signal of $67 \mathrm{mV}$ from the mixer corresponds to a phase change of $77^{\circ}$. From these data, where the response time of the phase shifter is dominated by the 90 ns rise time of the voltage pulse, one infers that the response time to a step function voltage would be equal to or less than the delay time, namely, approximately $30 \mathrm{~ns}$. This could be interpreted to correspond to an average

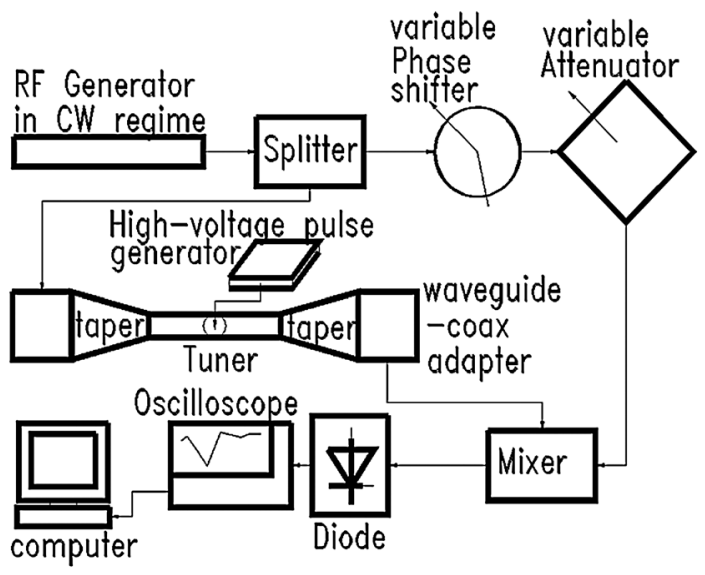

FIG. 10. The signal from the rf generator at $1290 \mathrm{MHz}$ is split in two. One portion is directed through a phase shifter and attenuator directly to a mixer, while the second portion is fed through the tuner input port, passes through the tuner, picked up at the tuner output port, and then is fed to the mixer. The resulting signal from the mixer is detected by a diode and monitored at an oscilloscope, and also captured by a computer for further signal processing [mainly via fast Fourier transformation (FFT)].

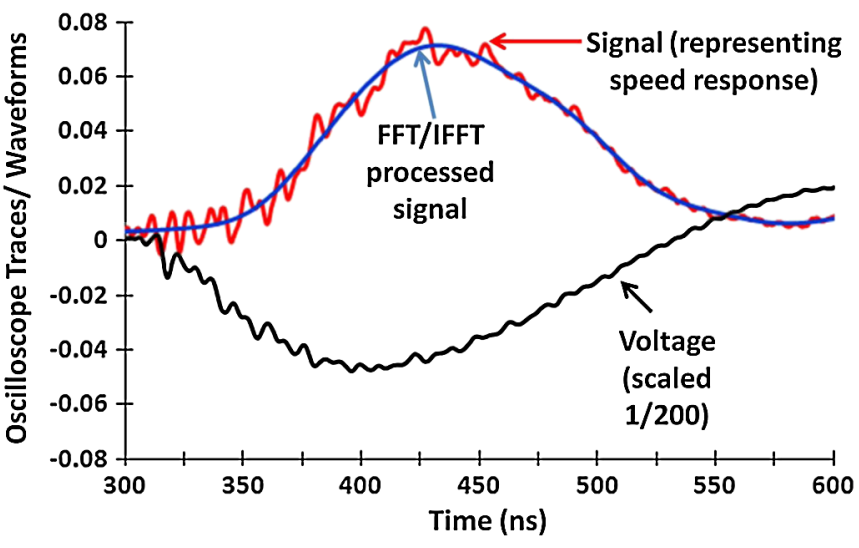

FIG. 11. Time response of phase shifter. Convex curve with wiggles (red) is the signal which is the difference between data with rf off and rf on. Smooth convex curve (blue) is the result of FFT/IFFT processing. The concave black curve is the highvoltage pulse with its peak value of $\sim 9.7 \mathrm{kV}$. It is seen that the time delay between the peak voltage and the peak variation in phase is $28 \mathrm{~ns}$. This value excludes delays in cables. The difference signal of $67 \mathrm{mV}$ from the mixer corresponds to a phase change of $77^{\circ}$. (One horizontal division is $50 \mathrm{~ns}$.)

switching rate of less than $0.5 \mathrm{~ns}$ for each degree of $\mathrm{rf}$ phase.

\section{OTHER DESIGNS}

As it has been mentioned, we considered several other designs for the phase shifter. Because of the nature of the article we shall describe them briefly here. 
Figure 12 presents the coaxial design. The ring (\#5) is ferroelectric material that is preceded by the matching ceramic (\#4). The gap is inevitable between the central part and the outer shell to apply the bias voltage. Consequently, one must employ a choke cavity (\#7). It is somewhat bulky design $(\varnothing 23 \mathrm{~cm} \times 56 \mathrm{~cm}$ height). It was found that the complexities and difficulties are hard to overcome when brazing both sides of the ferroelectric and/or insulation ceramic. Because of this, after a period of development we abandoned this version.

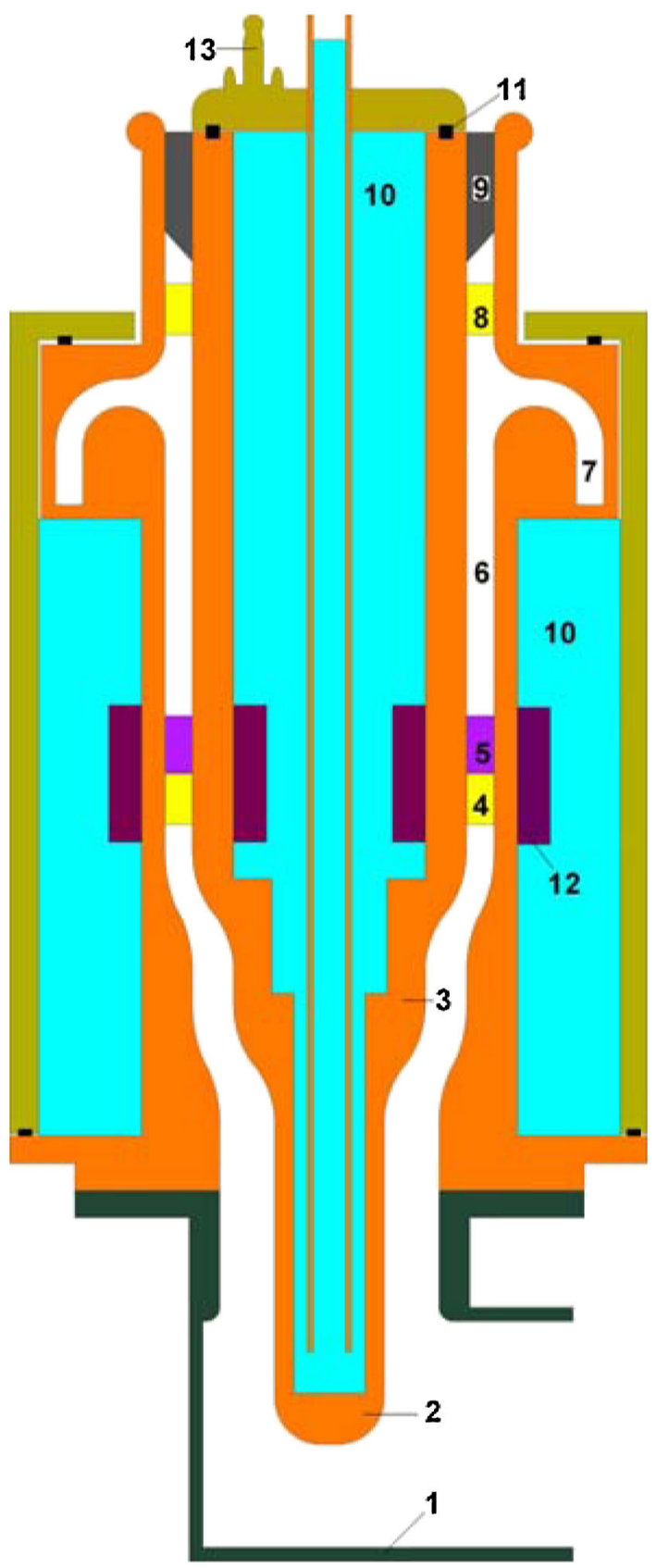

FIG. 12. Coaxial version (it is about $55 \mathrm{~cm}$ tall/ long, and $23 \mathrm{~cm}$ in diameter).
Figure 13 presents the planar-coax design. As before there is a gap between the cavity halves, and one must use a choke cavity. The ferroelectric ceramic (brown ring) is preceded by the matching ceramic (shown as yellow ring). The design is compact: $\varnothing 23 \mathrm{~cm} \times 12 \mathrm{~cm}$ height. Simulations of the full geometry show that the solution has many resonances in ferroelectric and matching ceramics. Unfortunately so far, we have failed to find a working solution for planar-coax geometry. However, the design is very attractive because of its simplicity. One may continue in two directions: (1) find a geometry modification in which the parasitic mode's frequencies are far from the working mode (if possible), and (2) carefully design the

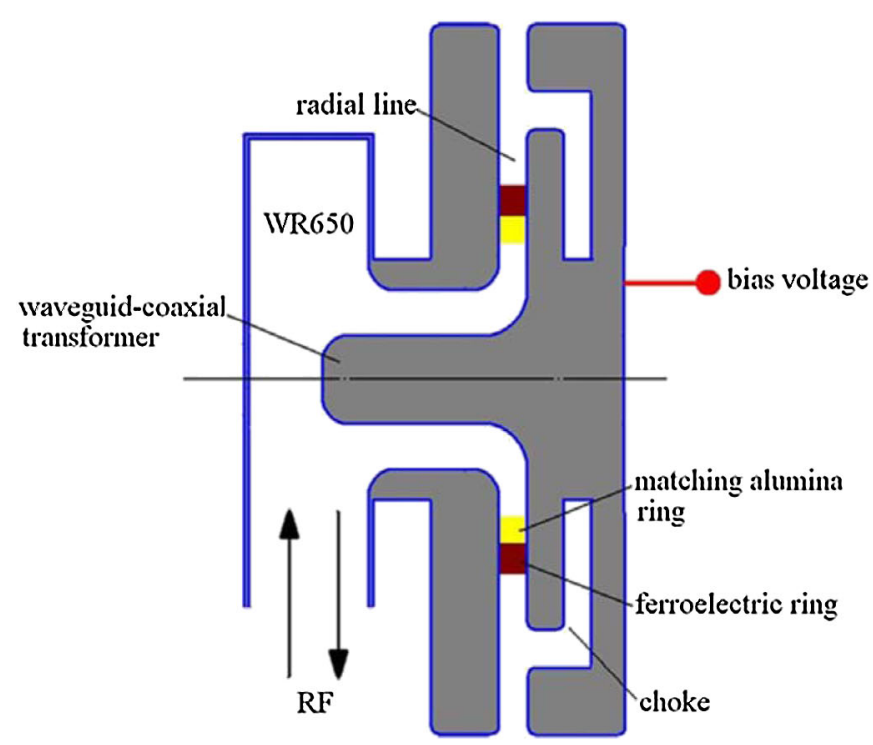

(a)

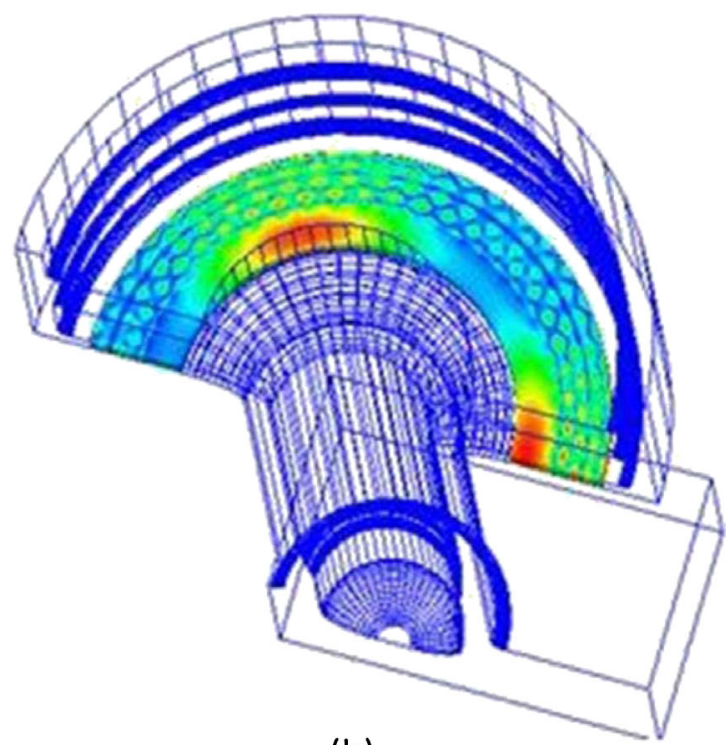

(b)

FIG. 13. (a) Planar-coax version (it is about $22 \mathrm{~cm}$ in diameter and $12 \mathrm{~cm}$ wide); and (b) example of a parasitic mode in the planar-coax version. 
coax-waveguide adapter in order not to excite parasitic modes. We should note that one of our co-authors has obtained a further monetary support to proceed in the aforementioned directions.

\section{CONCLUSIONS AND PLANS}

An rf-wave phase shifter based on a novel BST FE ceramic has been shown capable of delivering rapid phase switching (perhaps $<100 \mathrm{~ns}$ for shifts of $\sim 180$ degrees). That makes it an attractive candidate to externally tune the superconducting radiofrequency cavities for ERLs to reduce (at least by an order of magnitude) the rf-power requirements that arise because of phase instabilities of different origin, including microphonics as one of many.

The conducted research has revealed that several material issues must be addressed, including (a) redesigning the coating metallization layers; (b) brazing; (c) provisions to limit breakdowns at high bias voltages; and (d) redesigning the FE ceramic for L-band with low losses.

In a view of these issues, no further tests could be carried out, in particular until new improved FE ceramic samples with coating layers suitable for brazing would be delivered, but the schedule for this is uncertain.

The planned work also includes: (1) further developing the design for planar-coax geometry because it promises simplicity (relative to other designs) and thus low cost; and (2) proceeding with high-power tests but not necessary with the presented above $1 / 3$ model of the shifter. These efforts are underway.

Lastly, we note that the tuner in the configuration as described above was connected to a $1.3 \mathrm{GHz}$ cavity that is a mock-up of the superconducting rf-gun cavity designed to be used in the electron cooling project at BNL, see [11], and confirmed the capability of tuning of its resonance frequency [20].

\section{ACKNOWLEDGMENTS}

This work was supported by the Office of High Energy Physics, U.S. Department of Energy. We also acknowledge the help of Timergali Khabiboulline (FNAL), Harald Hahn (BNL), and E. M. Choi (formerly of BNL).

[1] S. Simrock et al., in Proceedings of the 20th Particle Accelerator Conference, Portland, OR, 2003 (IEEE, New York, 2003), p. 470.

[2] M. Liepe and S. Belomestnykh, in Proceedings of the 20th Particle Accelerator Conference, Portland, OR, 2003 (Ref. [1]), pp. 1326-1328.

[3] Y. Kang et al., in Proceedings of the 21st International Linac Conference, Gyeongju, Korea, 2002 (Pohang Accelerator Laboratory, Pohang, Korea, 2002), pp. 733-735.

[4] T. Zwart et al., in Proceedings of the 26th International Free Electron Laser Conference and 11th FEL User
Workshop, FEL 2004, Trieste, Italy, edited by René Bakker, Luca Giannessi, Marino Marsi, and Richard Walker (Comitato Conferenze Elettra, Trieste, Italy, 2004), pp. 542-545 [http://www.elettra.trieste.it/fel2004/ proceedings/].

[5] G. K. Davis and J. R. Delayen, in Proceedings of the 20th Particle Accelerator Conference, Portland, OR, 2003 (Ref. [1]), p. 1383.

[6] O. Kugeler, at the ERL09: Energy Recovery Linac, 45th ICFA Beam Dynamics Workshop, 2009, Cornell University [http://edms.classe.cornell.edu/agenda/ contributionDisplay.py?contribId=40\&amp; sessionId= 2\&amp; confId=7].

[7] D. Valuch et al., in Proceedings of the 9th European Particle Accelerator Conference, Lucerne, 2004 (EPSAG, Lucerne, 2004), pp. 959-961.

[8] D. Horan et al., in Proceedings of the 20th Particle Accelerator Conference, Portland, OR, 2003 (Ref. [1]), pp. 1177-1179.

[9] B. Foster et al., in Proceedings of the 21st Particle Accelerator Conference, Knoxville, 2005 (IEEE, Piscataway, NJ, 2005), p. 3123-3125.

[10] V. P. Yakovlev et al., in Proceedings of the 10th European Particle Accelerator Conference, Edinburgh, Scotland, 2006 (EPS-AG, Edinburgh, Scotland, 2006), pp. 487-489.

[11] I. Ben-Zvi, in Proceedings of the 10th European Particle Accelerator Conference, Edinburgh, Scotland, 2006 (Ref. [10]), pp. 940-944; See also R. Calaga, "SRF Cavities for High Current ERLs," BNL, 2006.

[12] O. A. Nezhevenko and V. P. Yakovlev, IEEE Transactions On Plasma Science, 2000, Vol. 28, No. 3 (IEEE Nuclear and Plasma Sciences Society, 2000), pp. 542-549 [http:// ieexplore.ieee.org/servlet/opac?punumber=27].

[13] D. Wang et al., in Proceedings of the 20th Particle Accelerator Conference, Portland, OR, 2003 (Ref. [1]), pp. 1300-1302.

[14] M. Liepe, at the ERL09: Energy Recovery Linac, 45th ICFA Beam Dynamics Workshop, 2009, Cornell University [http://edms.classe.cornell.edu/agenda/ contributionDisplay.py?contribId=37\&amp;sessionId= 2\&amp; confId=7].

[15] L.C. Sengupta, International Microwave Symposium, Boston, Massachusetts, 2000 [http://my.ece.ucsb.edu/ yorklab/Projects/Ferroelectrics/IMS2000\%20Workshop/ WFE002.pdf].

[16] D. Webb, International Microwave Symposium, Boston, Massachusetts, 2000 [http://my.ece.ucsb.edu/yorklab/ projects/Ferroelectrics/IMS2000\%20Workshop/ WFE004.pdf].

[17] A. B. Kozyrev et al., Tech. Phys. Lett. 24, 755 (1998).

[18] A. Kanareykin et al., in Proceedings of the 10th European Particle Accelerator Conference, Edinburgh, Scotland, 2006 (Ref. [10]), pp. 3251-3253.

[19] S. Yu. Kazakov et al., in Proceedings of the 2007 Particle Accelerator Conference, Albuquerque, New Mexico (IEEE, Albuquerque, New Mexico, 2007), pp. 596-598.

[20] H. Hahn et al., in Proceedings of the 23rd Particle Accelerator Conference, Vancouver, Canada, 2009 (IEEE, Piscataway, NJ, 2009) [http://trshare.triumf.ca/ $\sim$ pac09proc/Proceedings/papers/tu5pfp035.pdf]. 


\section{Omega-P Fast X-Band Switch Design Review}

\section{Holmes}

January 31, 2007 


\section{Outline}

- Design Ground Rules and Assumptions

- Supplied Geometry and Schematic

- System Assembly

- System Components

- Assembly Sequence

- Summary 


\section{Ground Rules and Assumptions}

- UHV waveguide at $11.4 \mathrm{GHz}$

- 2 cavities loaded with ferroelectric ceramic ring

- Switch testing requires biasing the center section up to $100 \mathrm{kV}$

- Power density and dissipation is the same as for the phase shifter

- Configured for testing at SLAC with SLED-II pulse compressors (38.1 mm circular wave guide)

- Tapered adapters required for testing at NRL

- Ceramics must be replaceable

- Cavities must be tunable (disassemble \& machine)

- Bakeout up to $250^{\circ} \mathrm{C}$ required

- No fluid connections in vacuum 


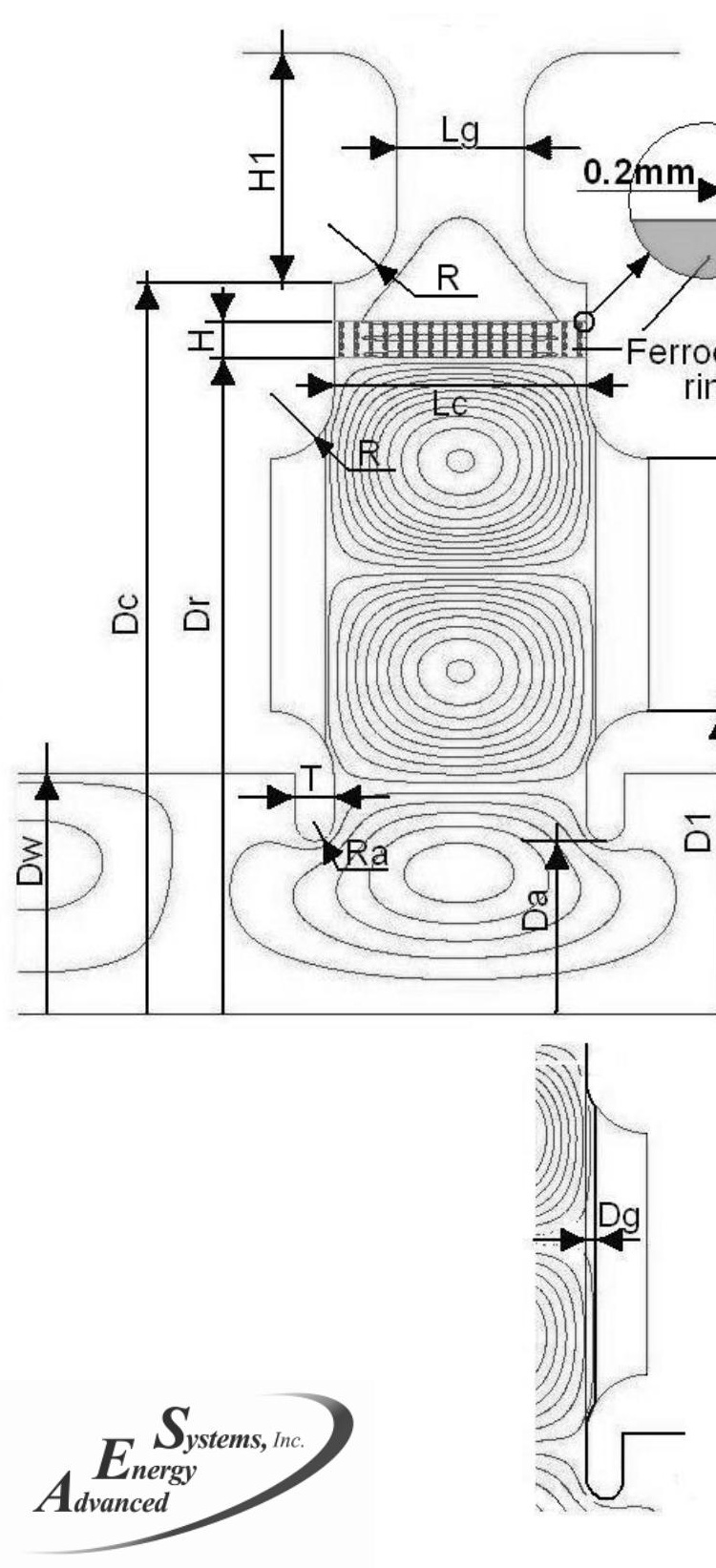

\begin{tabular}{|c|c|c|}
\hline geometrical parameter & $\begin{array}{c}\text { nominal } \\
\text { value, mm }\end{array}$ & $\begin{array}{c}\text { derivation } \\
\mathrm{MHz} / \mu\end{array}$ \\
\hline internal diameter of the ferroelectric ring, $\mathrm{D}_{\mathrm{r}}$ & 104.2 & $\mathrm{df} / \mathrm{dD}_{\mathrm{r}}=-0.055$ \\
\hline ferroelectric ring thickness, $\mathrm{H}^{*}$ & 2.7 & $\mathrm{df} / \mathrm{dH}=-0.2$ \\
\hline ferroelectric ring length, $L_{r}$ & 20.4 & - \\
\hline cavity length, $\mathrm{L}_{\mathrm{c}}$ & 20 & $\mathrm{df} / \mathrm{dD}_{\mathrm{c}}=-0.16$ \\
\hline cavity diameter, $\mathrm{D}_{\mathrm{c}}$ & 115.8 & - \\
\hline width of the slot, $\mathrm{L}_{\mathrm{g}}$ & 10 & - \\
\hline radial size of the slot, $\mathrm{H}_{1}$ & 30 & - \\
\hline coupling iris diameter, $\mathrm{D}_{\mathrm{a}}$ & 27.4 & $\mathrm{df} / \mathrm{dD}_{\mathrm{a}}=-0.03$ \\
\hline lower diameter of the tuning groove, $\mathrm{D}_{1}$ & 38 & $\mathrm{df} / \mathrm{dD}_{1}=+0.05$ \\
\hline upper diameter of the tuning groove, $\mathrm{D}_{2}$ & 92 & $\mathrm{df} / \mathrm{dD}_{2}=-0.05$ \\
\hline groove rounding radius, $\mathrm{R}$ (the same for both & 5 & $\mathrm{df} / \mathrm{dR}=-0.12$ \\
\hline coupling diaphrages) & 3 & $\mathrm{df} / \mathrm{dT}=$ \\
\hline radius of the coupling iris rounding, $\mathrm{R}_{\mathrm{a}}$ & 1.5 & +0.015 \\
\hline waveguide diameter, $\mathrm{D}_{\mathrm{w}}$ & 38.1 & - \\
\hline length of waveguide insertion, $\mathrm{L}_{\mathrm{w}}{ }^{* *}$ & 80.1 & - \\
\hline depth of the tuning grooves $\mathrm{D}_{\mathrm{g}}$ (initial) & 0 & \\
\hline
\end{tabular}




\section{Supplied Schematic}

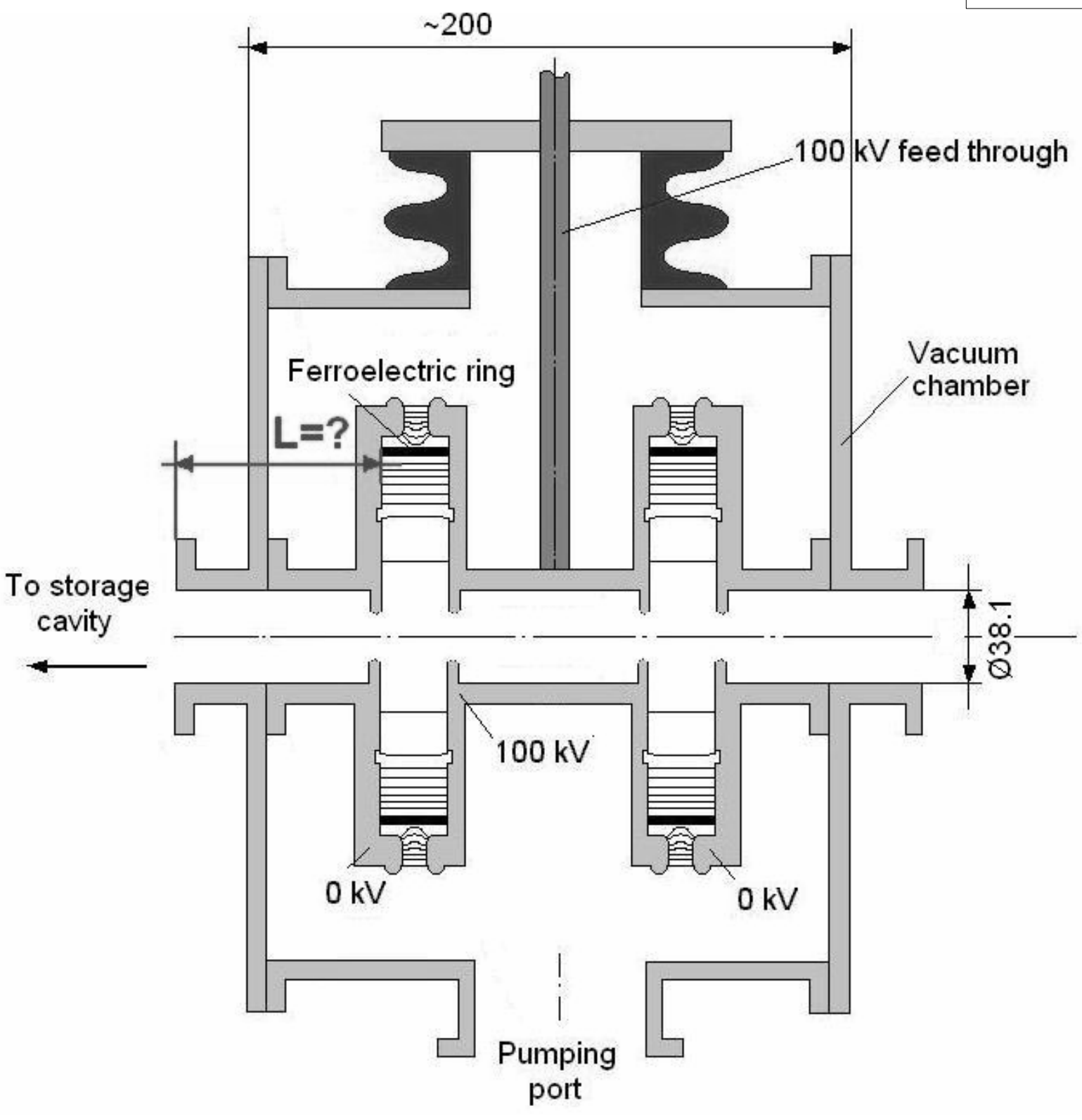

D. Holmes - X-Band Switch Design 1-31-07: page - 5 of 14 


\section{System Assembly}

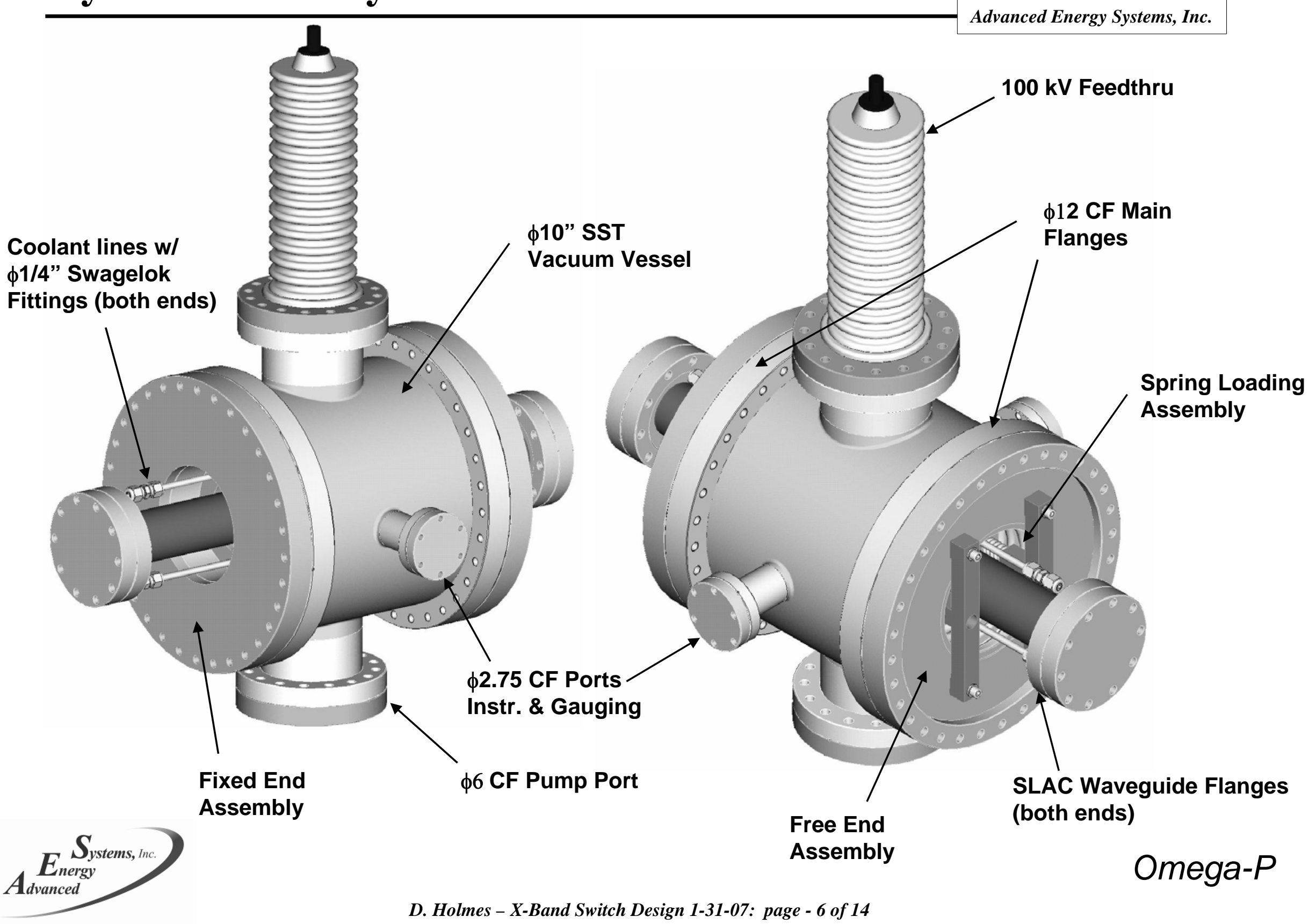




\section{Elevation View}
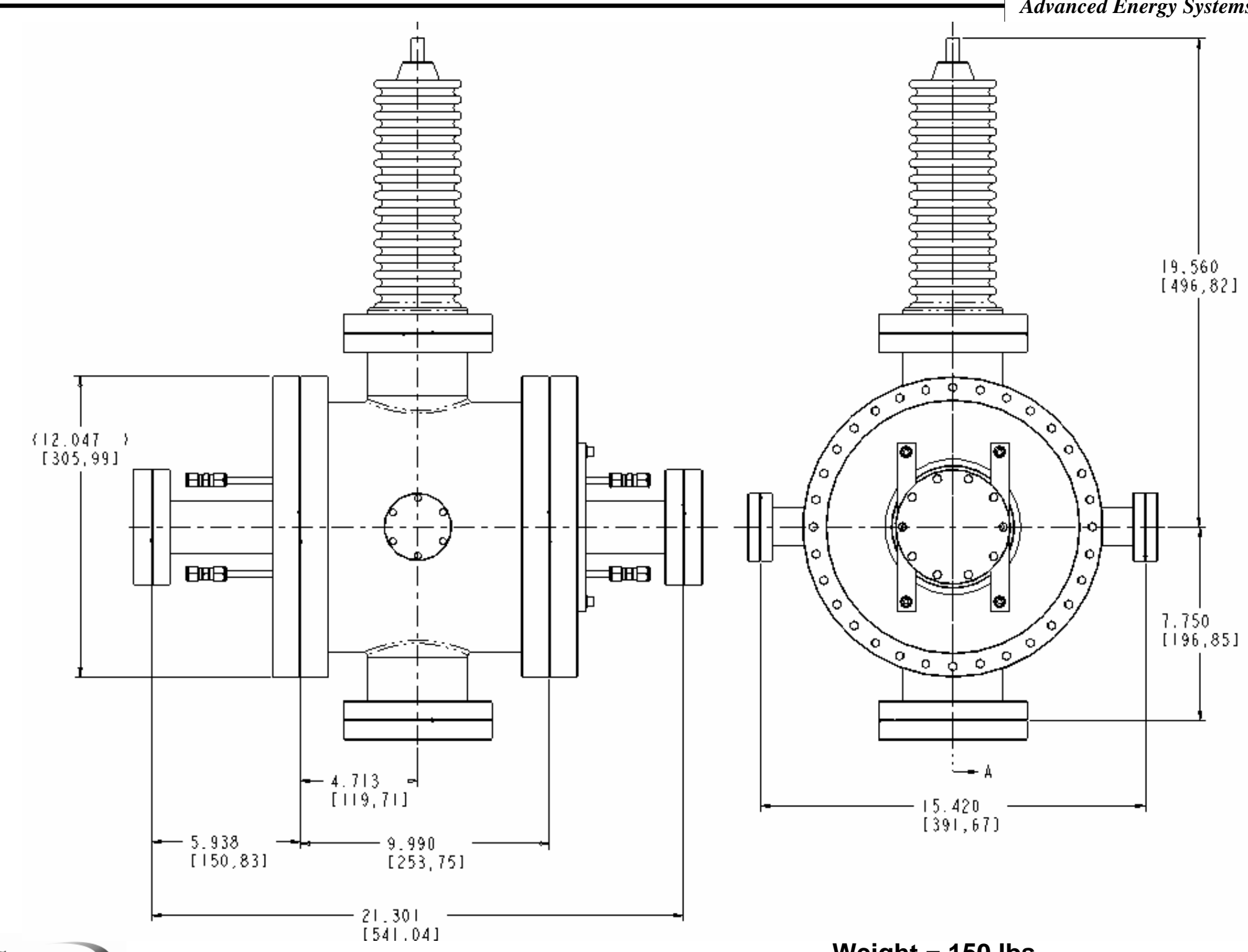

Weight $=150 \mathrm{lbs}$ 


\section{System Components}

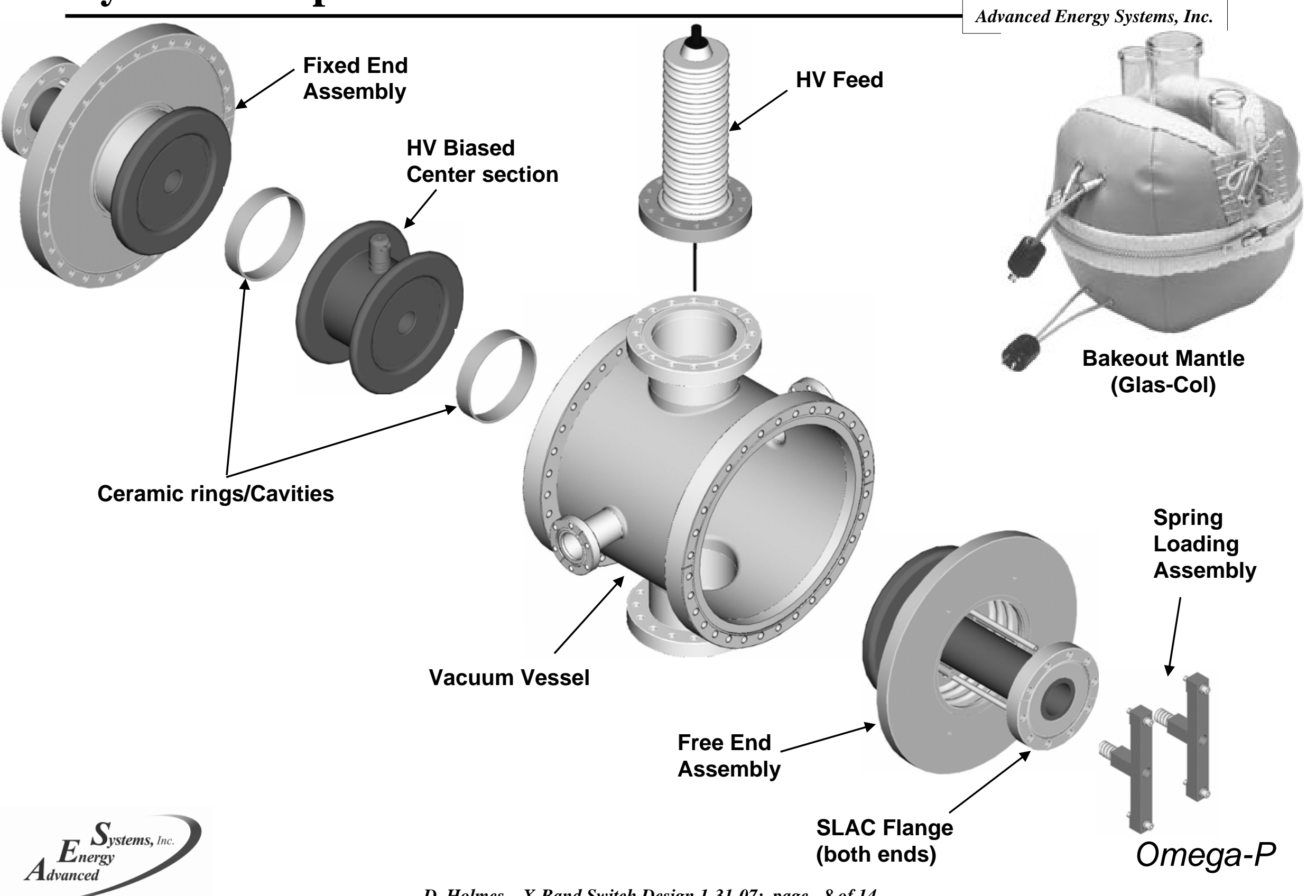




\section{Design - Section View}

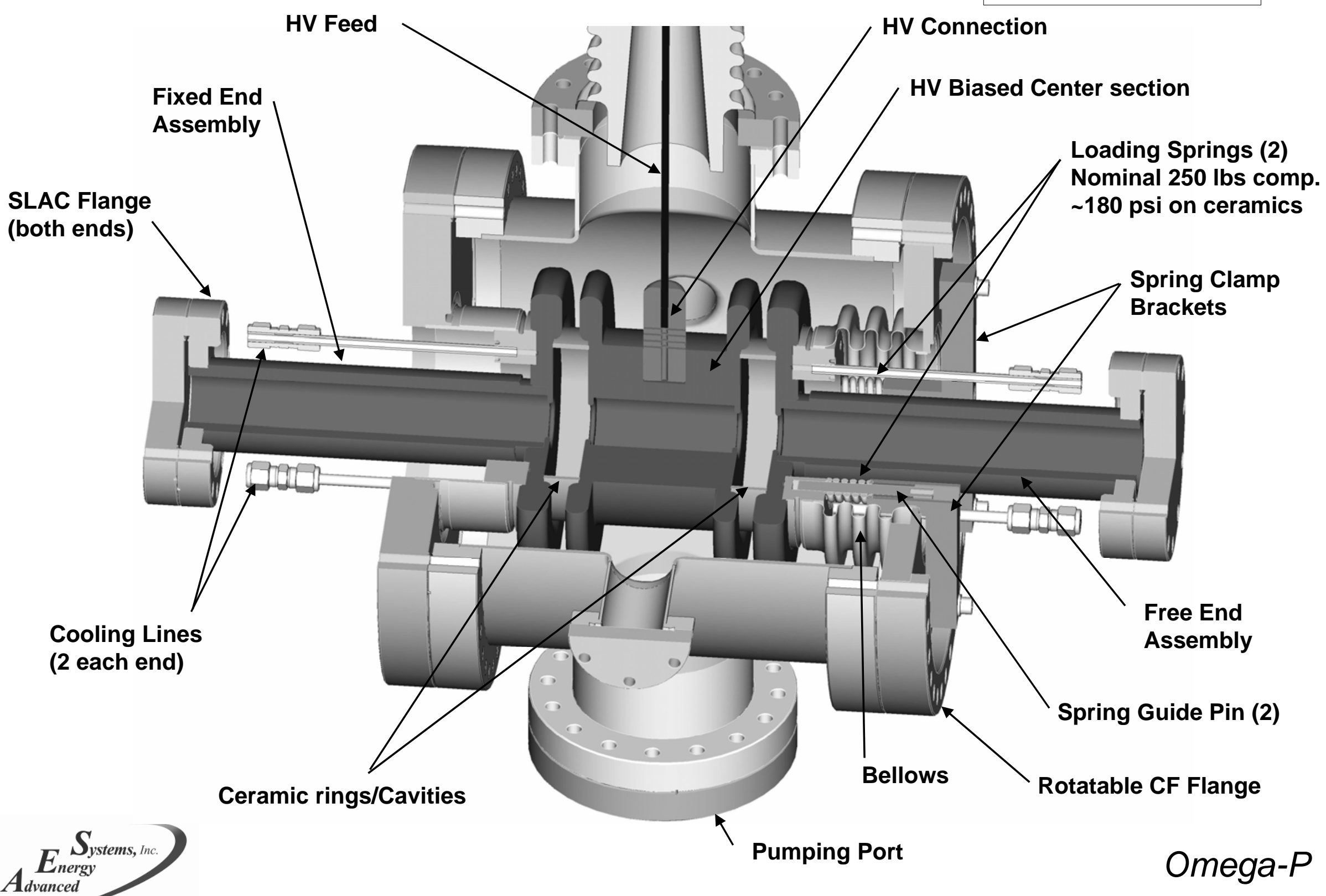

D. Holmes - X-Band Switch Design 1-31-07: page - 9 of 14 


\section{Assembly Sequence 1}

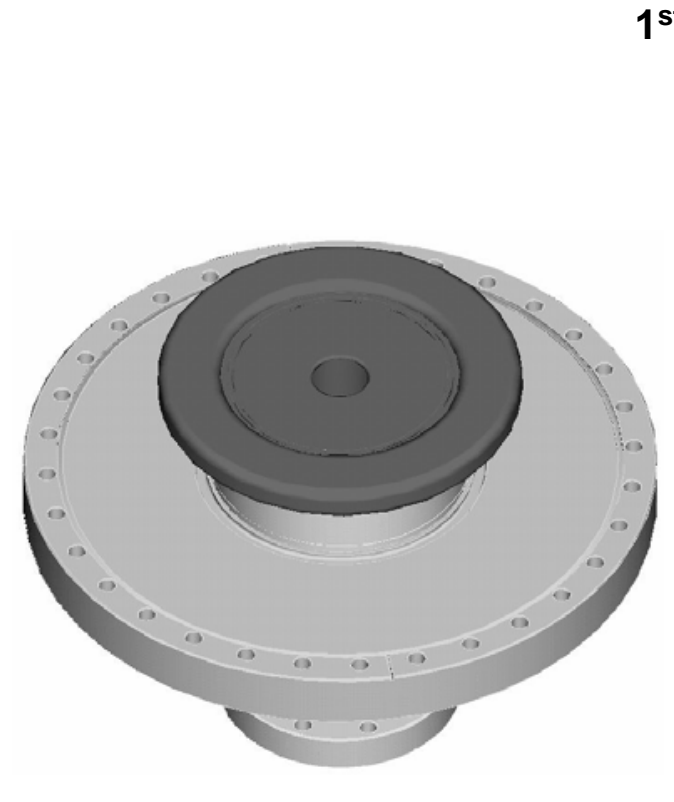

$1^{\text {st }}$ Ceramic Ring

\section{HV Center Section}
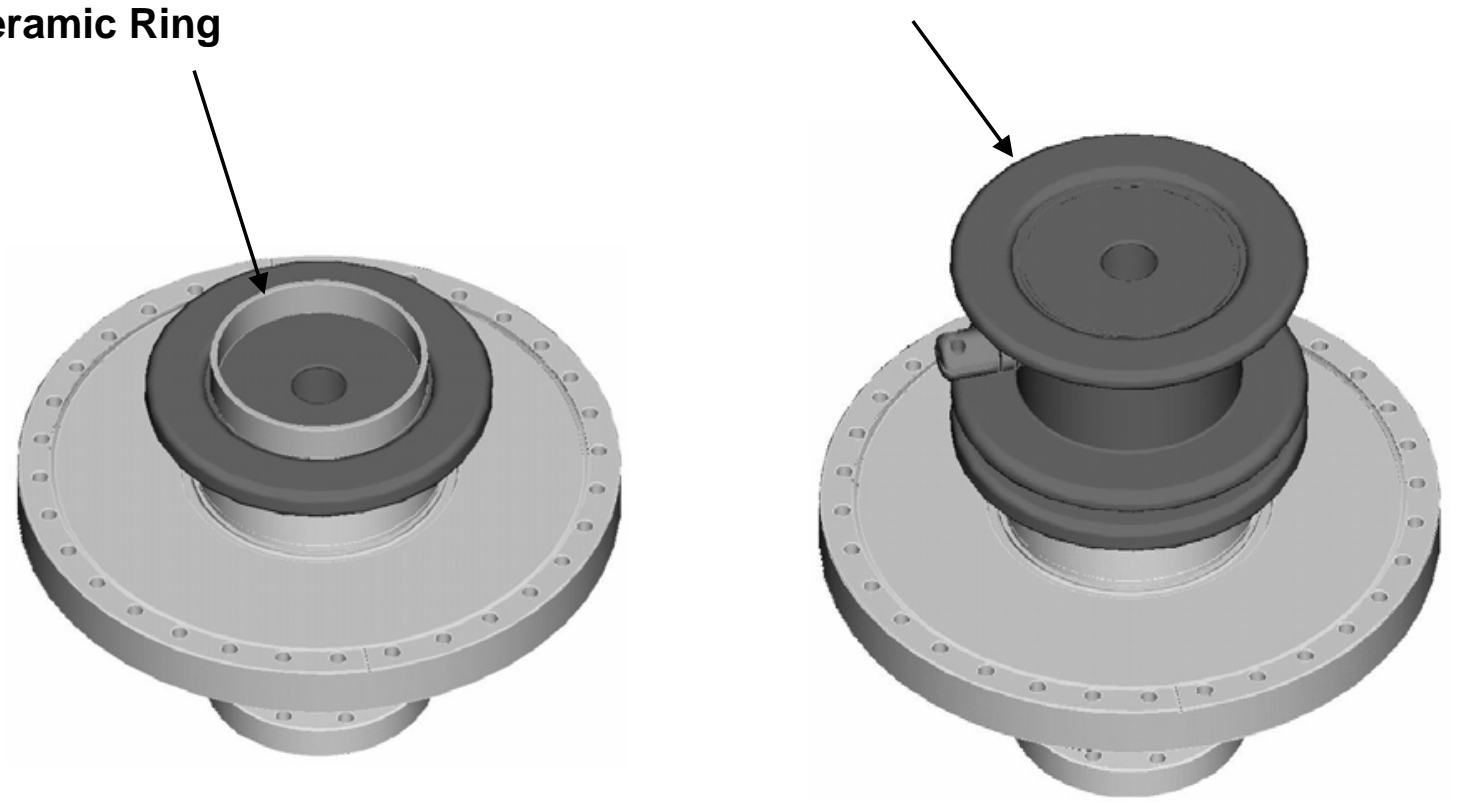

Fixed End Assembly

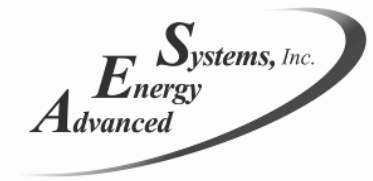




\section{Assembly Sequence 2}

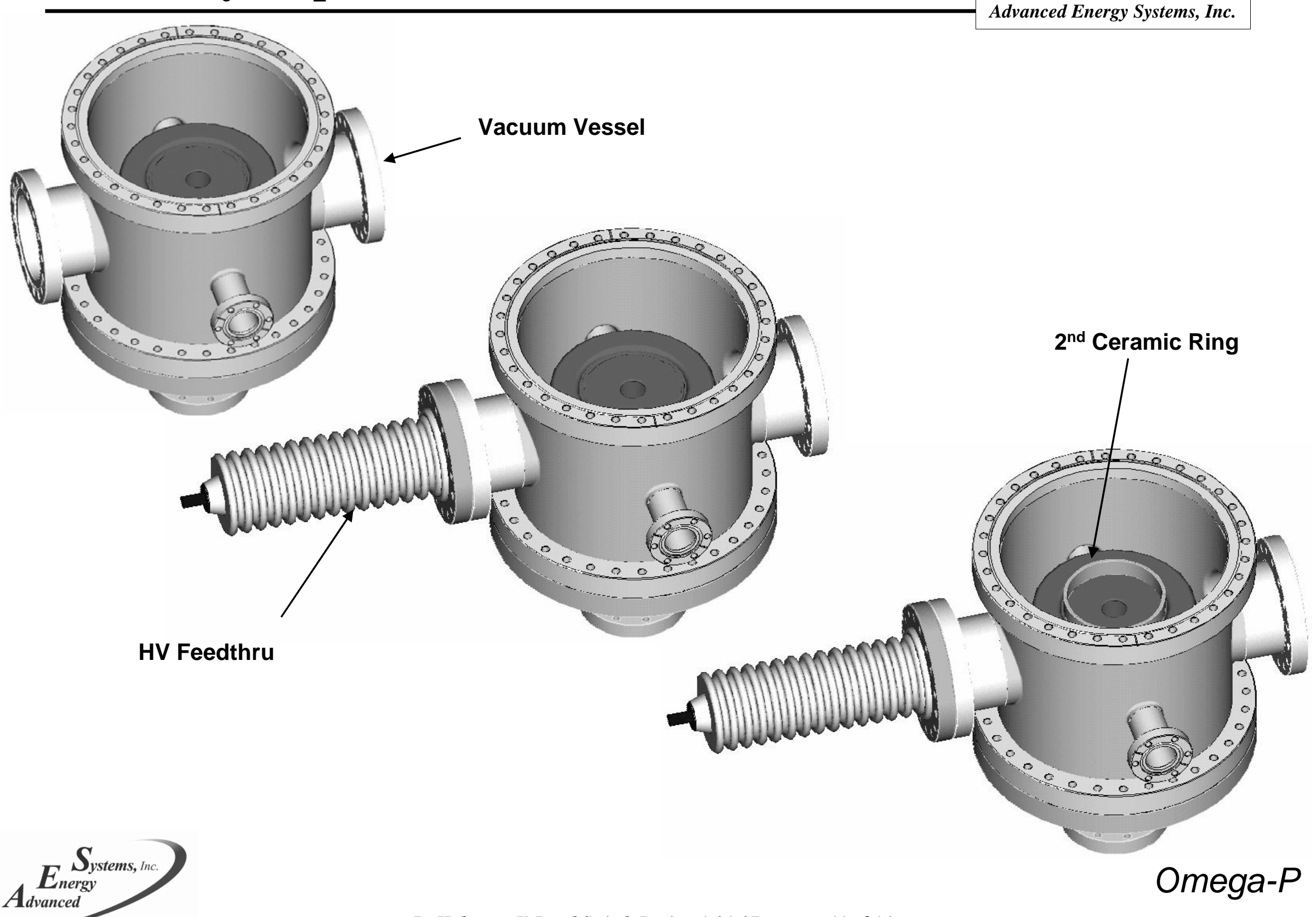




\section{Assembly Sequence 3}

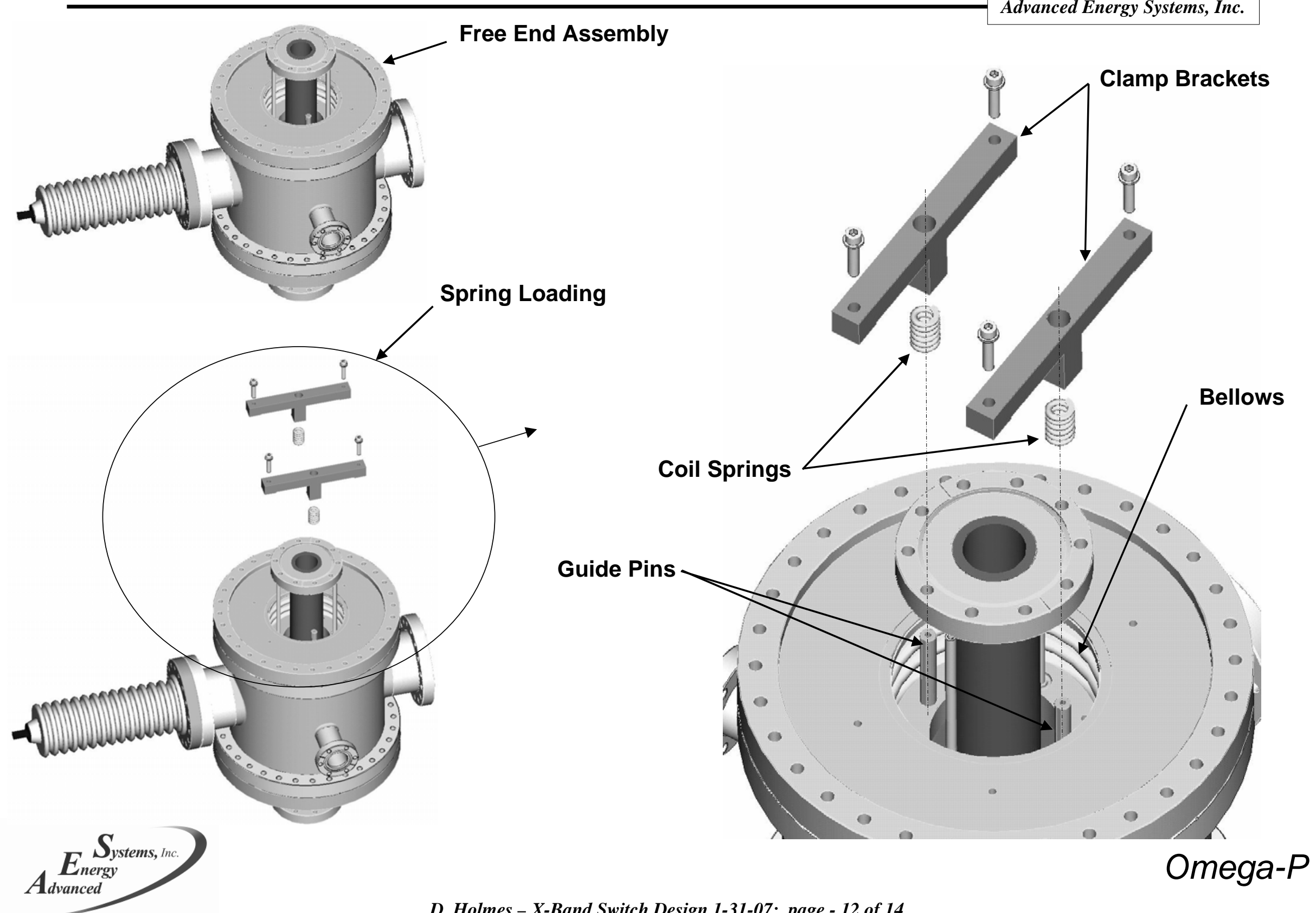




\section{Completed Assembly}

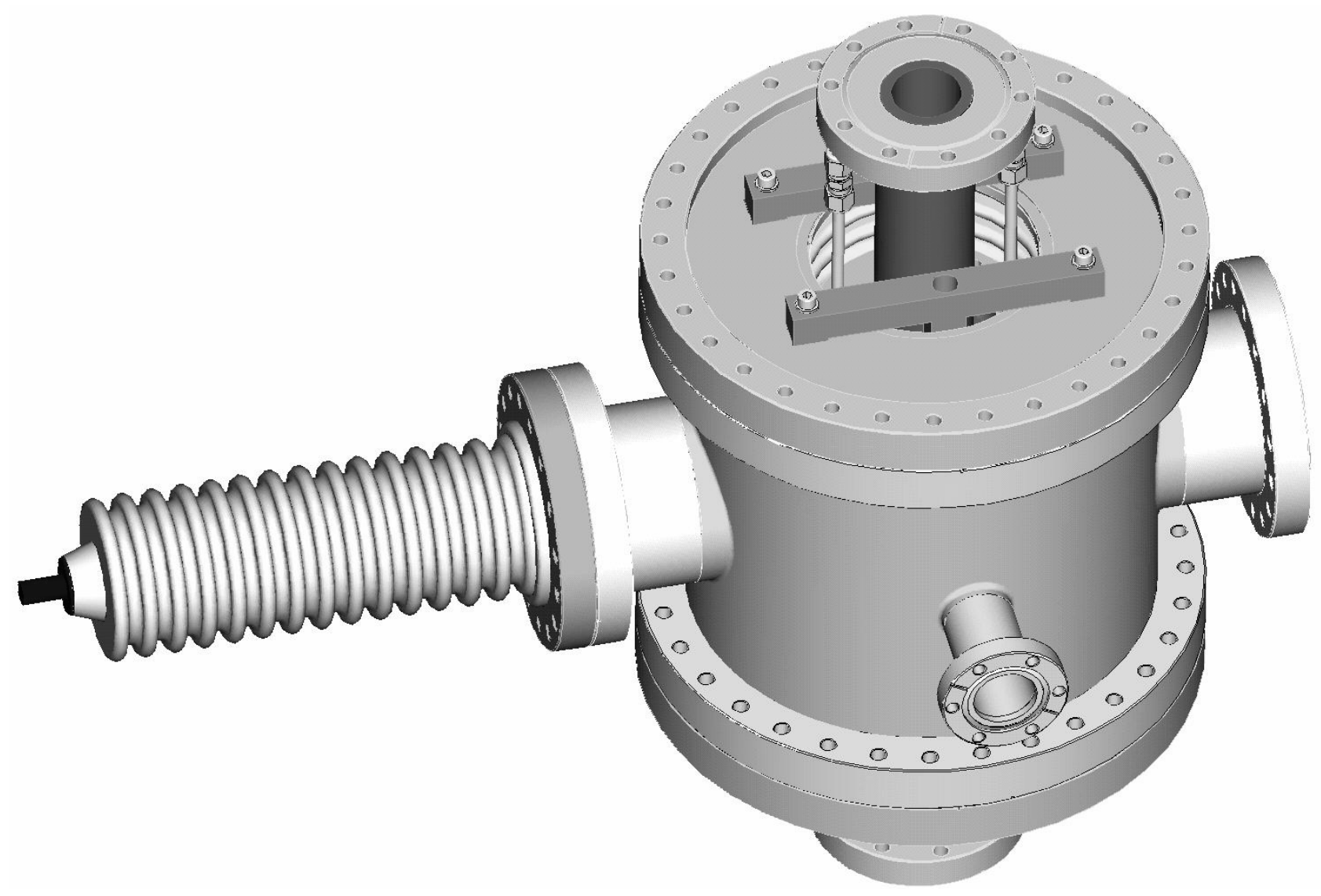




\section{Summary}

- Design uses no mechanical fittings in vacuum for cooling

- Cooling to vacuum braze joints are used.

- Direct cooling with channel integrated into grounded cavity halves

- HV center section is conduction cooled through ceramic

- Spring loading provides compression load on assembly while allowing for differential expansion during bakeout and operation

- Vertical assembly is done on fixed end wall and the vacuum housing is used for support and spring loading the assembly 


\title{
X-Band High-Power Fast FERROELECTRIC SWITCH*
}

\author{
V.P. Yakovlev ${ }^{1,2}$, S. Shchelkunov 3 , \\ A. Kanareikin ${ }^{4}$, E. Nenasheva ${ }^{5}$, J.L. Hirshfield ${ }^{1,3}$ \\ ${ }^{1}$ Omega-P, Inc., New Haven, CT 06511, USA \\ ${ }^{2}$ Fermi National Accelerator Laboratory, IL, USA \\ ${ }^{3}$ Physics Department, Yale University, New Haven, CT 06520, USA \\ ${ }^{4}$ Euclid Techlabs LLC, Solon, OH 44139. USA \\ ${ }^{5}$ Ceramics Ltd., St. Petersburg, 194223, Russia
}

in collaboration with

Steven Gold 6

${ }^{6}$ Naval Research Lab, Washington, USA

${ }^{*}$ This work is supported by DoE, office of HEP 
- The current designs for linear colliders relies on pulse compression to achieve high peak power levels in relatively short pulses [e.g. for NLS it is $\sim 500 \mathrm{MW}$ in $\sim 400 \mathrm{nsec}]$

- Among RF-compression systems, two are considered, namely:

a) Resonant Delay Line Pulse Compression System

b) Delay Line Distribution System (DLDS)

\section{PROBLEM(s)}

- Passive circuitry (so to speak), and thus low compression ratios ( 4:1)

- DLDS (passive version) requires $\sim 100 \mathrm{~km}$ of waveguides

\section{POSSIBLE SOLUTION:}

- Active DLDS, whose key element is a high power microwave switch 


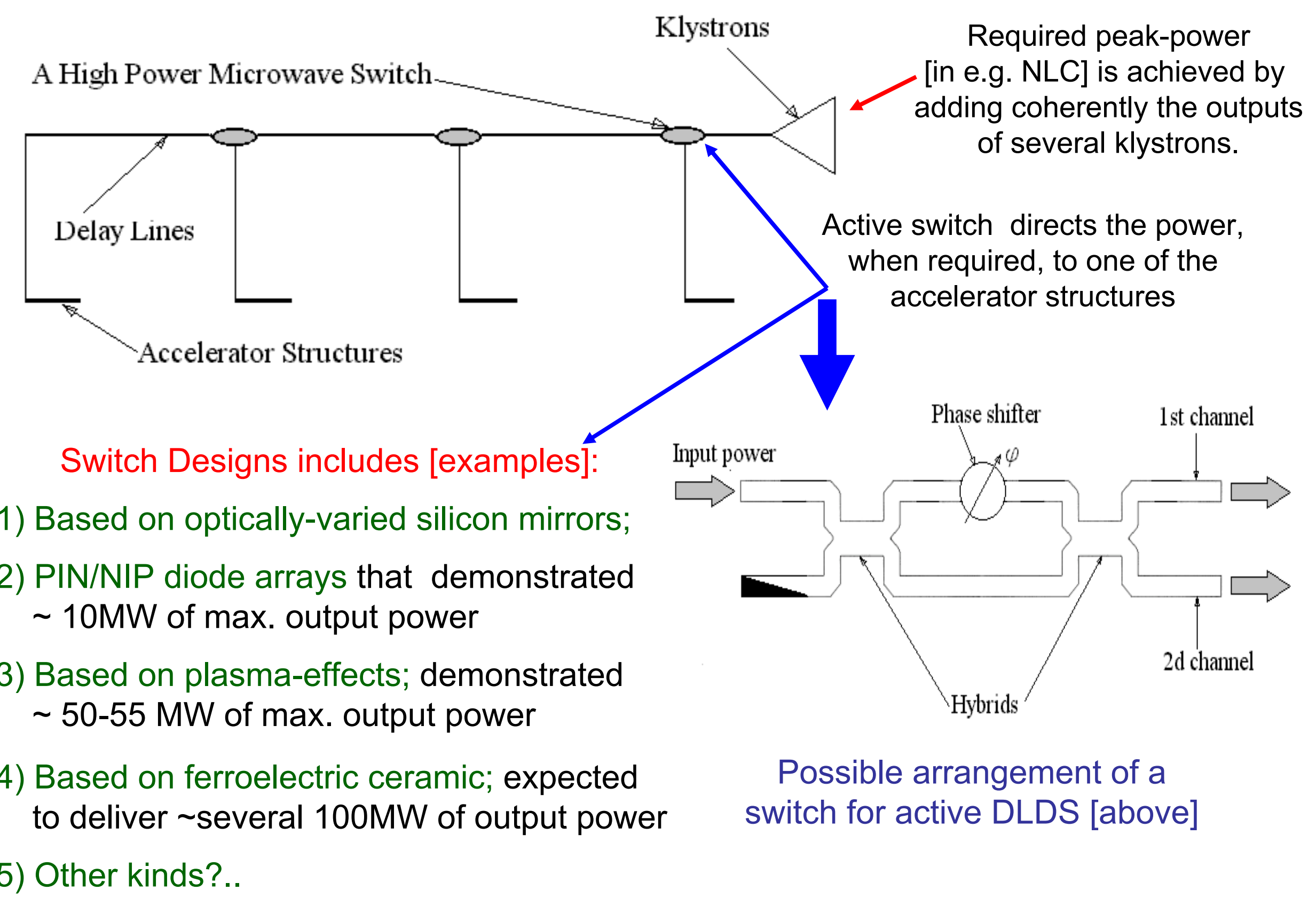


...the high power phase shifter that is the main component of a fast ferroelectric-based microwave switch as shown in the previous slide appears to be too complex and expensive to built when one considers the proof-ofprinciple experiment...

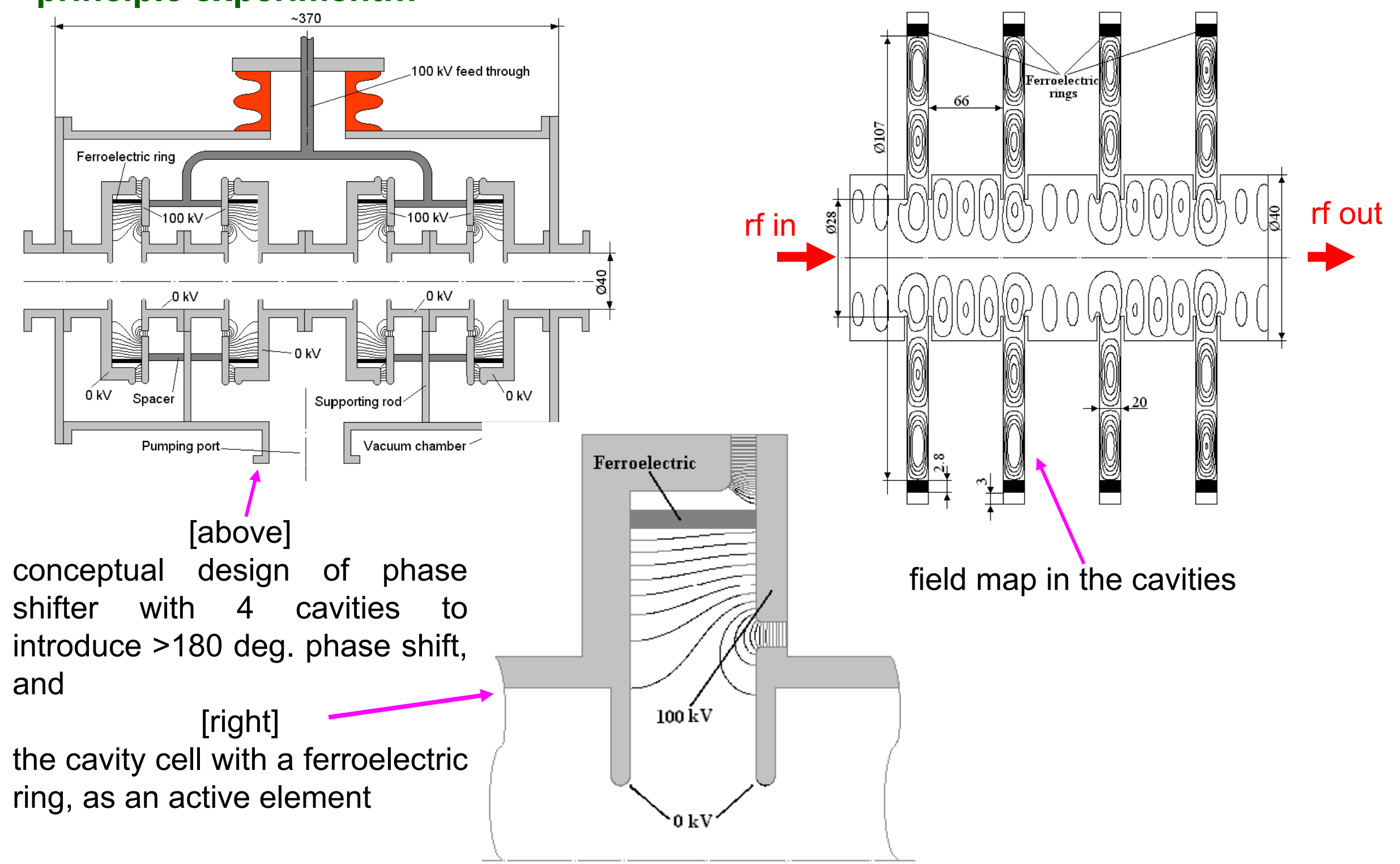


- Built a relatively simple and inexpensive model that clearly demonstrates the abilities to work as a fasts switch as a standalone device without needs for hybrids or magic-tees.

- A design emerged that is basically a two-cell switch cavity that uses the same principles as the phase shifter; yet alone can change the coupling between two rf-elements, for example: the feed-line and a cavity.

- The device is to be tested as a part of Active Bragg Compressor (ABC-2 at NRL), and if successful will imply that more complex designs as presented before are feasible, thus paving the future for fast high-power active phase shifters/switches to be used e.g. for active DLDS.

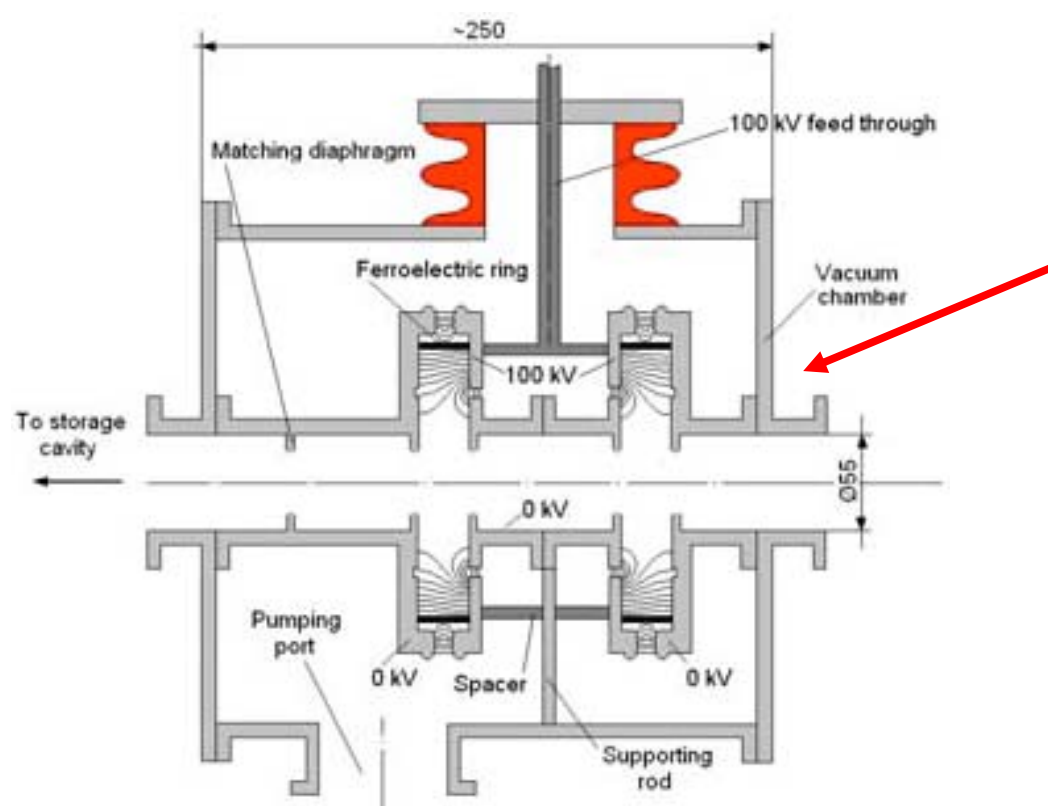

the 2-cell switch cavity [named X-Band HighPower Fast Ferroelectric Switch] resembles the 4-cavity phase shifter mentioned before, and uses the BST-ceramic as an active element [ see the next page ] 
Properties of BST ceramic, which is proposed as an active medium for ferroelectric based switches/ tuners/ phase shifters

\begin{tabular}{|l|c|}
\hline dielectric constant, $\varepsilon$ & $\sim 500$ \\
\hline tunability, $\partial d \partial \mathrm{E}_{\text {bias }}\left(\mathrm{E}_{\text {bias }}\right.$ is the bias field $)$ & $\sim 1.2-1.5 /(\mathrm{kV} / \mathrm{cm})$ \\
\hline response time & $<10 \mathrm{~ns}$ \\
\hline loss tangent at $11.4 \mathrm{GHz}, \operatorname{tg}(\delta)$ & $\sim 5 \times 10^{-3}$ \\
\hline breakdown limit & $200 \mathrm{kV} / \mathrm{cm}$ \\
\hline thermal conductivity, $K$ & $7.02 \mathrm{~W} / \mathrm{m}-{ }^{\circ} \mathrm{K}$ \\
\hline specific heat, $C$ & $0.605 \mathrm{~kJ} / \mathrm{kg}^{\circ} \mathrm{K}$ \\
\hline density, $\rho$ & $4.86 \mathrm{~g} / \mathrm{cm}^{3}$ \\
\hline coefficient of thermal expansion & $10.1 \times 10^{-6} /{ }^{\circ} \mathrm{K}$ \\
\hline temperature tolerance, $\partial \varepsilon \partial \mathrm{T}$ & $3 /{ }^{\circ} \mathrm{K}$ \\
\hline
\end{tabular}




\section{Examples of ferroelectric material: rings, bars by Euclid} Tech-Labs LLC
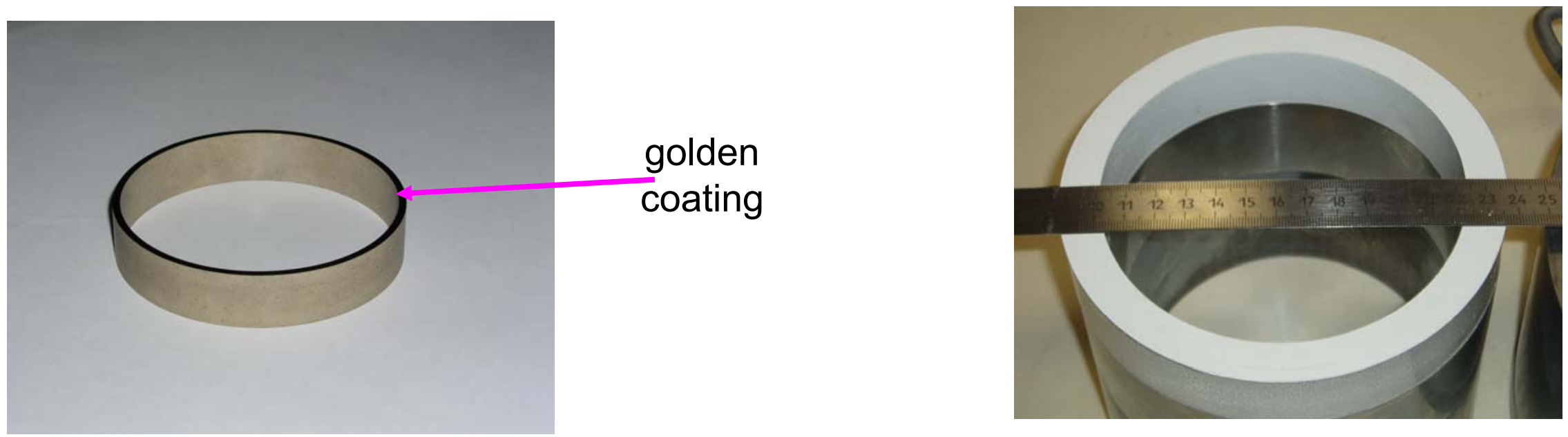

diameter $=106 \mathrm{~mm}$; thickness $=2.8 \mathrm{~mm}$; length $=22 \mathrm{~mm}$

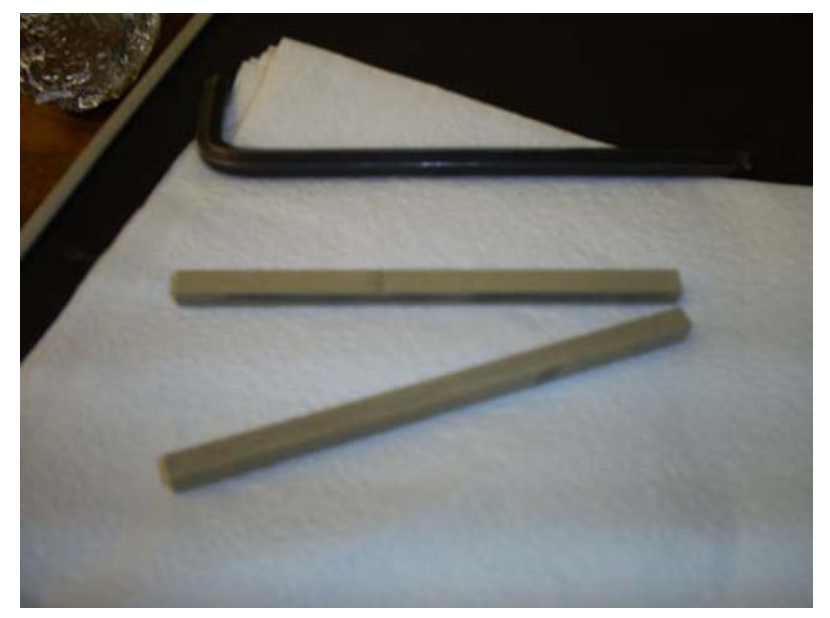

diameter $=106 \mathrm{~mm}$; thickness $=12 \mathrm{~mm}$; length $=22 \mathrm{~mm}$

width = $6 \mathrm{~mm}$;

height $=5 \mathrm{~mm}$;

length $=\sim 108 \mathrm{~mm}$ 
a) Measurements of loss of ferroelectric ceramic (BSM-3):

...measuring the $\mathrm{Q}$-factor for different frequencies ( $\mathrm{f}$ ), knowing the field distribution (from simulations), and using the law $\mathrm{Q} \times \mathrm{f} \approx$ const one derives the loss-tan, $\tan (\sigma) \ldots$

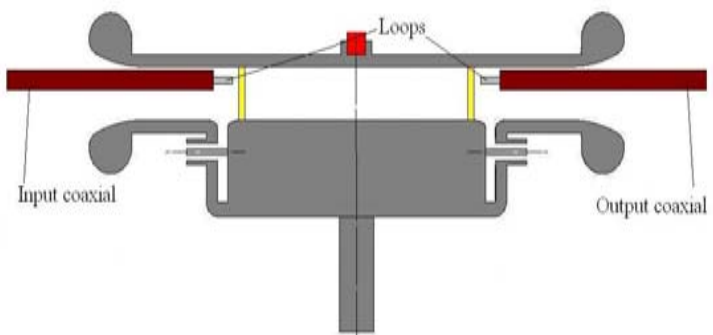

ring inside a specially designed test cavity

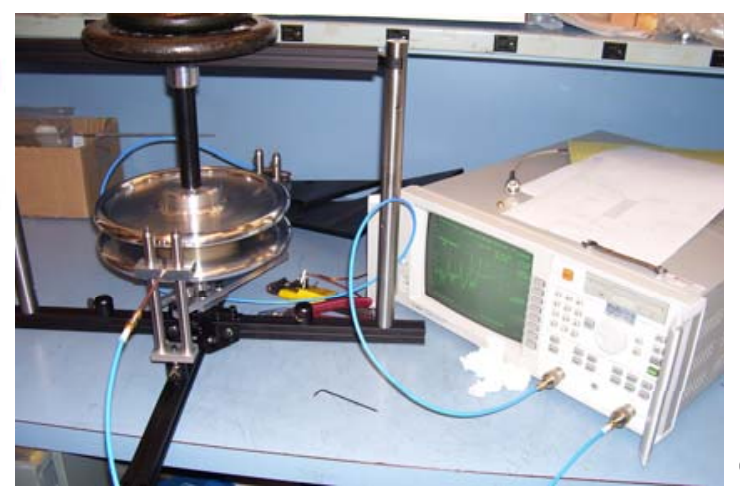

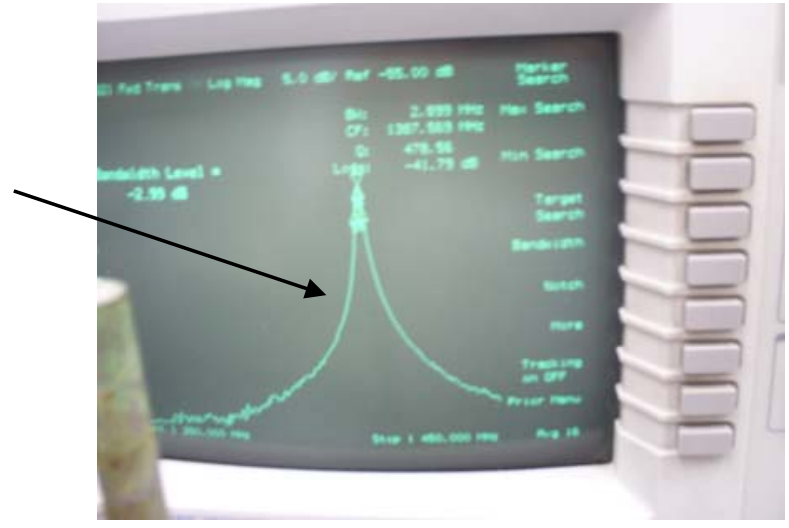

$f \sim 11.4 \mathrm{GHz}, \tan (\sigma) \sim 5 \cdot 10^{-3}$

...given the particular geometry of the switch, the losses are acceptable

b) Measurements of tunability of ferroelectric material (BSM-3):
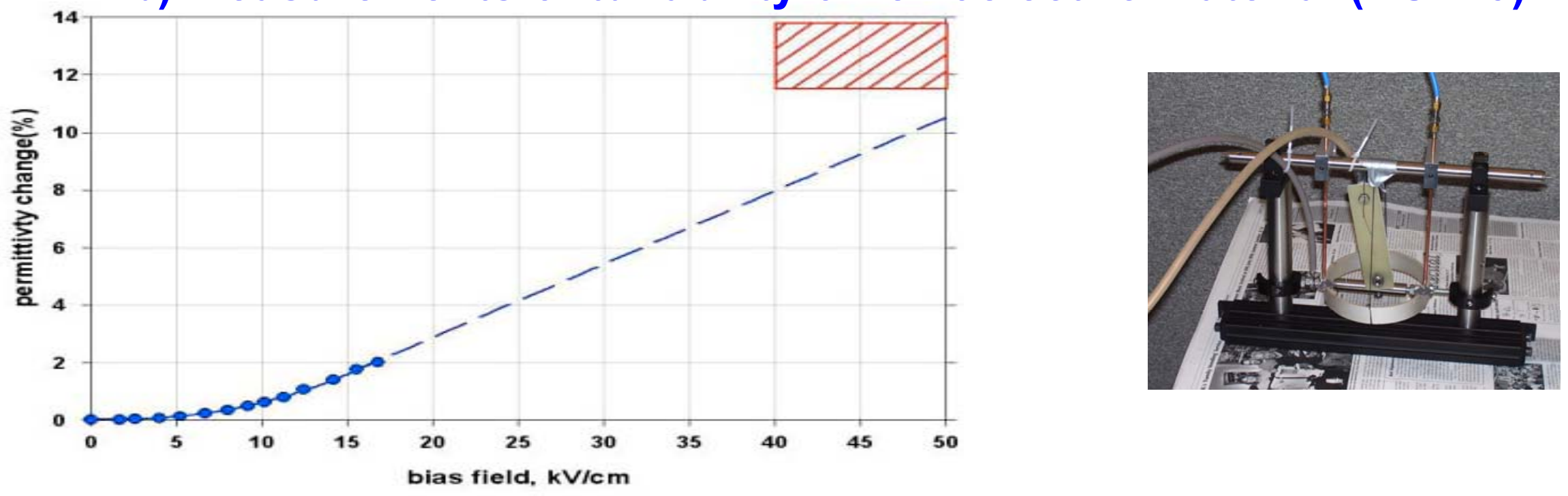

- large ring measurements 
...active pulse compressor (@NRL) will be for operating frequency $11.43 \mathrm{GHz}$; input power $\sim 20 \mathrm{MW}$, power gain $\sim 10$, and consequently output power $200 \mathrm{MW}$. Input pulse duration $\sim 0.5$ micro-sec, while the output pulse duration $\sim 40$ nsec.

\section{[ switch cavity parameters are listed below]}

Cavity 1 and 2 are identical

TABLE II. Parameters of $\mathrm{TE}_{031}$ switch cavity.

[see parameters in Table]

\begin{tabular}{|l||l|}
\hline Operating frequency, GHz & 11.424 \\
\hline operating mode & $\mathrm{TE}_{031}$ \\
\hline cavity length, mm & 20 \\
\hline cavity diameter, mm & 118 \\
\hline coupling iris diameter, mm & 30 \\
\hline coupling diaphragm thickness, mm & 3 \\
\hline ferroelectric ring length, mm & 20 \\
\hline ferroelectric ring inner diameter, mm & 106 \\
\hline ferroelectric ring thickness, mm & 3 \\
\hline transmission in energy extraction regime $(\varepsilon=400), \mathrm{dB}$ & 0 \\
\hline transmission in energy storage regime $(c=500), \mathrm{dB}$ & -4 \\
\hline maximum electric field in ferroelectric in energy extraction regime, $\mathrm{kV} / \mathrm{cm}$ & 20 \\
\hline maximum electric field in ferroelectric in energy storage regime, $\mathrm{kV} / \mathrm{cm}$ & 10 \\
\hline maximum electric field in vacuum in energy extraction regime, $\mathrm{kV} / \mathrm{cm}$ & 865 \\
\hline maximum electric field in vacuum in energy storage regime, $\mathrm{kV} / \mathrm{cm}$ & 675 \\
\hline
\end{tabular}

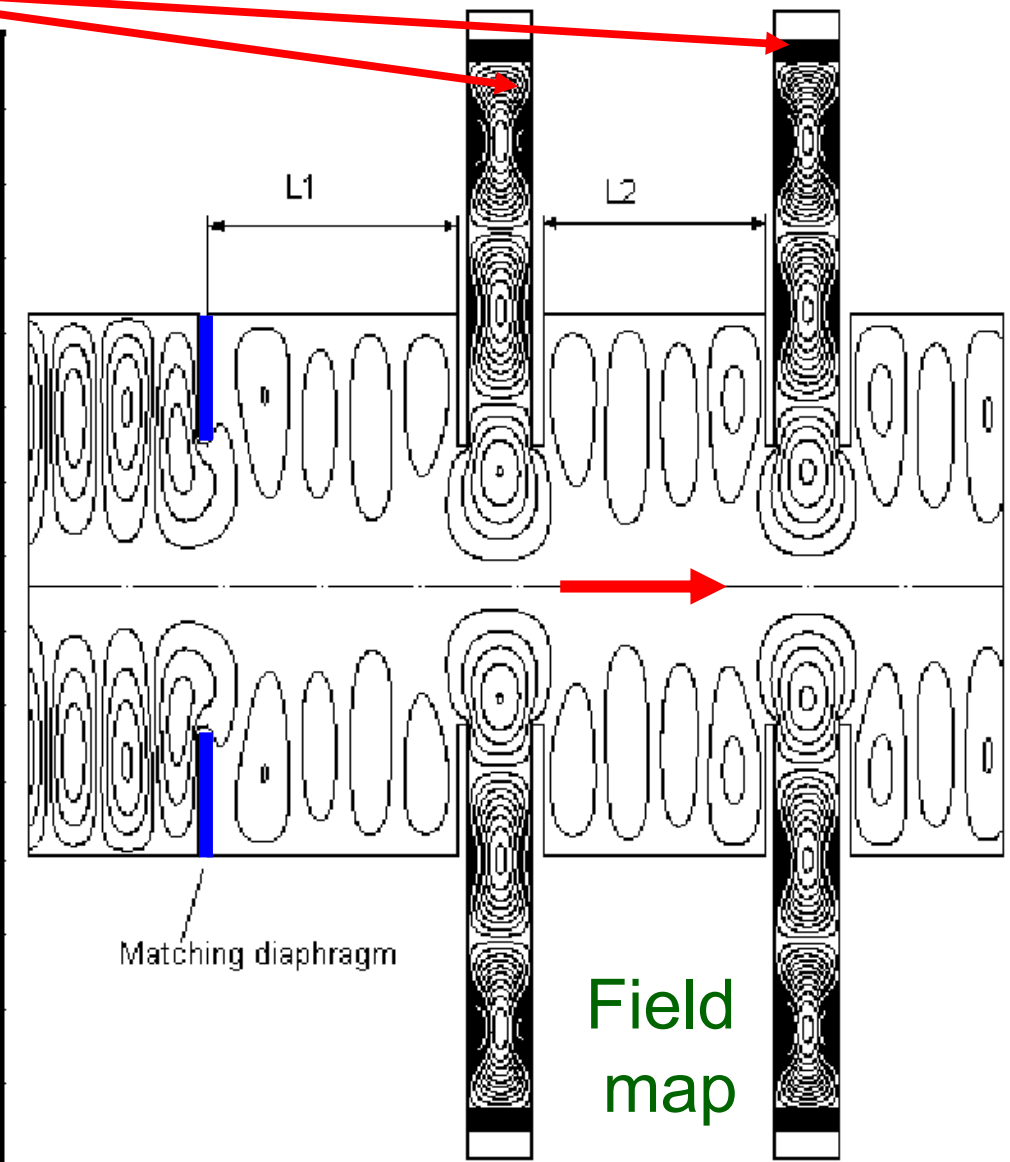




\section{Design was developed...}

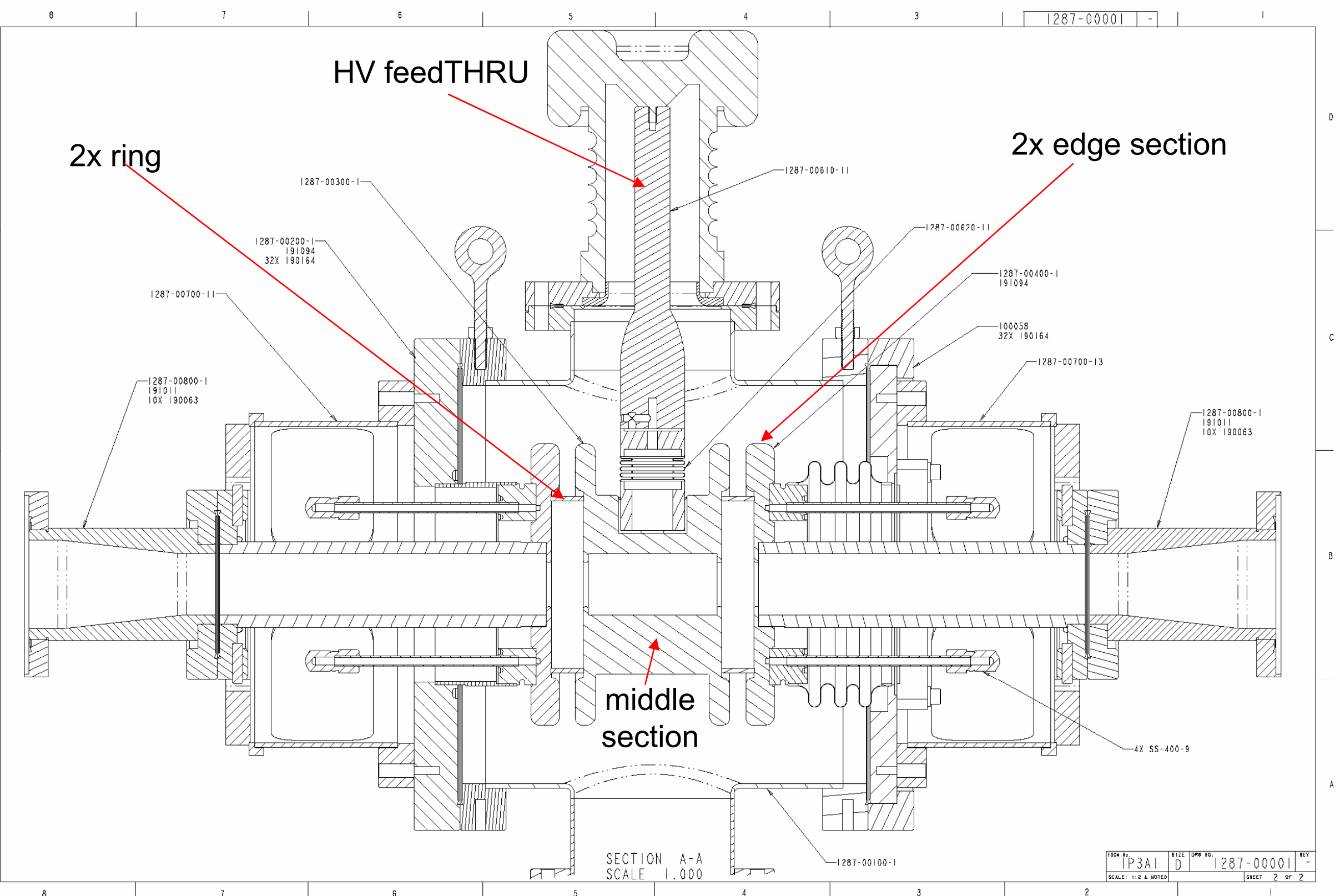


Parts were machined, the rings were manufactured... (structure is not yet assembled)
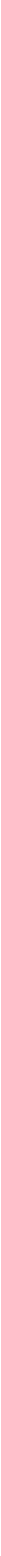
To tune the structure (by machining the grooves in designated places, several auxiliary items were made, but some of them [e.g. modelaunchers] also should be adjusted/tuned to work properly...
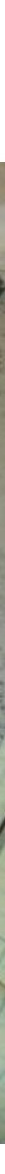


\section{Conclusions}

- Designs for X-band phase shifter and X-band switch were presented

- X-band switch is simulated, designed, and its parts are manufactured

- Ceramic rings are manufactured

- X-band switch must be assembled, vacuum checked, and tuned to frequency

- Auxiliary items

- are manufactured

- some items must be tuned, but the work is complicated by the absence of an $\sim 11 \mathrm{GHz}$ network analyzer

- X-band switch test

- must be shipped to NRL, installed to the circuitry, and operated... 


\section{X-Band High-Power Fast FERROELECTRIC SWITCH*}

\section{S. Shchelkunov ${ }^{1}$, Steven Gold ${ }^{2}$ V.P. Yakovlev ${ }^{3}$, J.L. Hirshfield ${ }^{1,4}$}

${ }^{1}$ Physics Department, Yale University, New Haven, CT 06520, USA

${ }^{2}$ Naval Research Lab, Washington, USA

${ }^{3}$ Fermi National Accelerator Laboratory, IL, USA

${ }^{4}$ Omega-P, Inc., New Haven, CT 06511, USA

*This work is supported by DoE, office of HEP 


\section{MOTIVATION (Part 1):}

- The current designs for linear colliders relies on pulse compression to achieve high peak power levels in relatively short pulses [e.g. for NLS it is $\sim 500 \mathrm{MW}$ in $\sim 400 \mathrm{nsec}]$

- Among RF-compression systems, two are considered, namely:

a) Resonant Delay Line Pulse Compression System

b) Delay Line Distribution System (DLDS)

\section{PROBLEM(s)}

- Passive circuitry (so to speak), and thus low compression ratios ( 4:1)

- DLDS (passive version) requires $\sim 100 \mathrm{~km}$ of waveguides

\section{POSSIBLE SOLUTION:}

- Active DLDS, whose key element is a high power microwave switch 


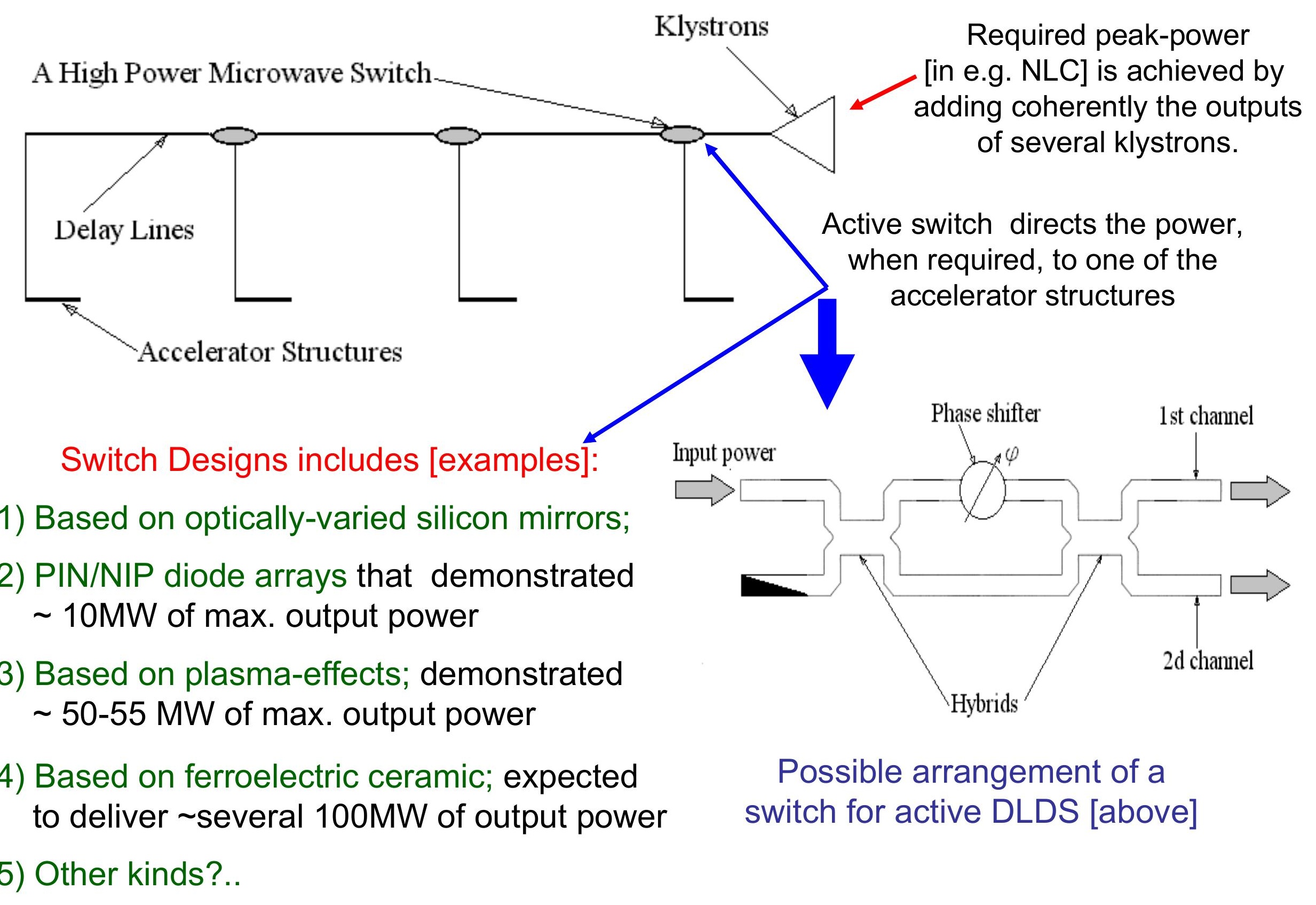


...the high power phase shifter that is the main component of a fast ferroelectric-based microwave switch as shown in the previous slide appears to be too complex and expensive to built when one considers the proof-ofprinciple experiment...

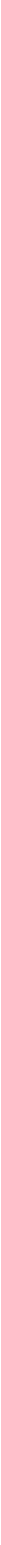




\section{MOTIVATION (Part 2):}

- Built a relatively simple and inexpensive model that clearly demonstrates the abilities to work as a fasts switch as a standalone device without needs for hybrids or magic-tees.

- A design emerged that is basically a two-cell switch cavity that uses the same principles as the phase shifter; yet alone can change the coupling between two rf-elements, for example: the feed-line and a cavity.

- The device is to be tested as a part of a compression scheme (at NRL), and if successful will imply that more complex designs as presented before are feasible, thus paving the future for fast high-power active phase shifters/switches to be used e.g. for active DLDS.

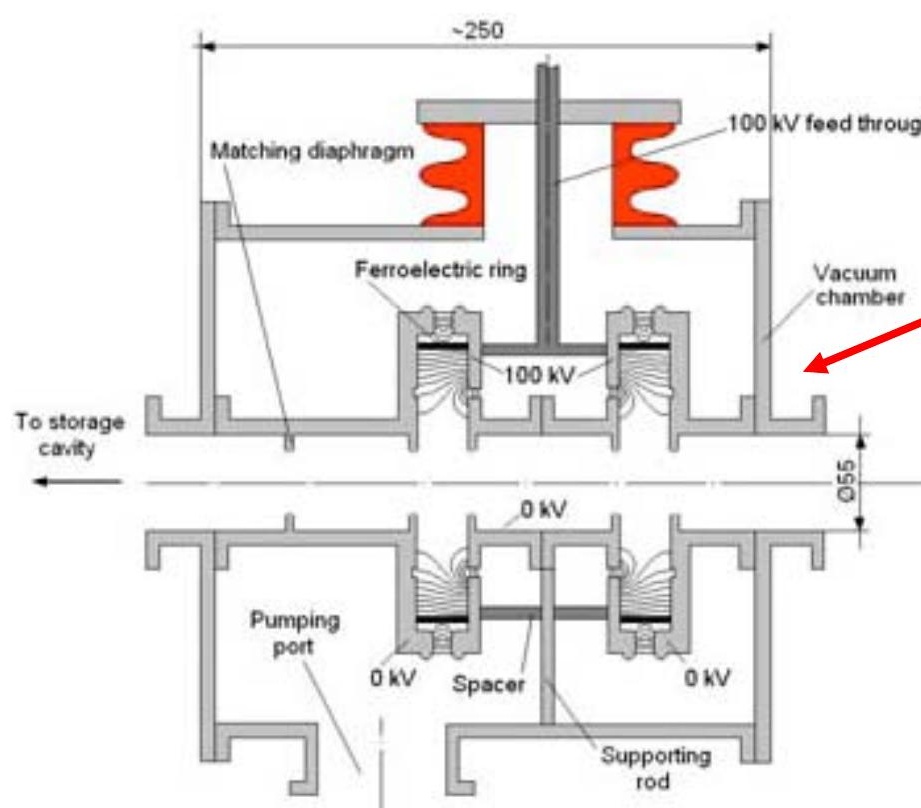

the 2-cell switch cavity [named X-Band HighPower Fast Ferroelectric Switch] resembles the 4-cavity phase shifter mentioned before, and uses the BST-ceramic as an active element [ see the next page ] 
Properties of BST ceramic, which is proposed as an active medium for ferroelectric based switches/ tuners/ phase shifters

\begin{tabular}{|l|c|}
\hline dielectric constant, $\varepsilon$ & $\sim 500$ \\
\hline tunability, $\partial \varepsilon / \partial \mathrm{E}_{\text {bias }}\left(E_{\text {bias }}\right.$ is the bias field $)$ & $\sim 1.2-1.5 /(\mathrm{kV} / \mathrm{cm})$ \\
\hline response time & $<10 \mathrm{~ns}$ \\
\hline loss tangent at $11.4 \mathrm{GHz}, \operatorname{tg}(\delta)$ & $\sim 5 \times 10^{-3}$ \\
\hline breakdown limit & $200 \mathrm{kV} / \mathrm{cm}$ \\
\hline thermal conductivity, $K$ & $7.02 \mathrm{~W} / \mathrm{m}-{ }^{\circ} \mathrm{K}$ \\
\hline specific heat, $C$ & $0.605 \mathrm{~kJ} / \mathrm{kg}^{\circ} \mathrm{K}$ \\
\hline density, $\rho$ & $4.86 \mathrm{~g} / \mathrm{cm}^{3}$ \\
\hline coefficient of thermal expansion & $10.1 \times 10^{-6} /{ }^{\circ} \mathrm{K}$ \\
\hline temperature tolerance, $\partial \varepsilon \partial \mathrm{T}$ & $3 /{ }^{\circ} \mathrm{K}$ \\
\hline
\end{tabular}


Examples of ferroelectric material: rings, bars by Euclid Concepts LLC, OH ( A. Kanareikin, et al )

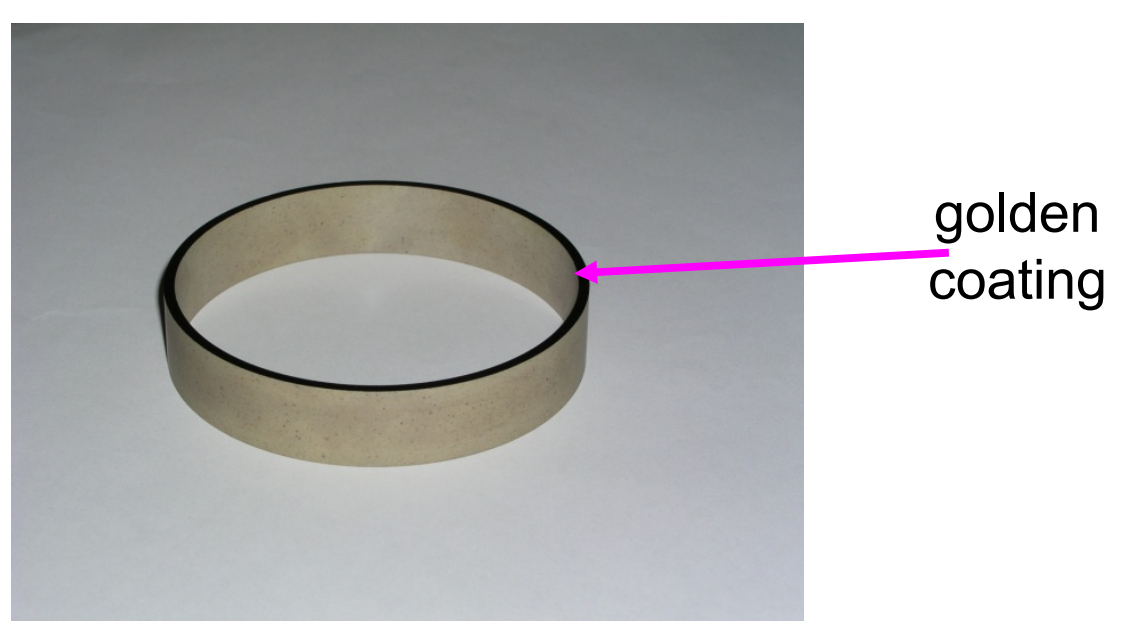

diameter $=106 \mathrm{~mm}$; thickness $=2.8 \mathrm{~mm}$; length $=22 \mathrm{~mm}$

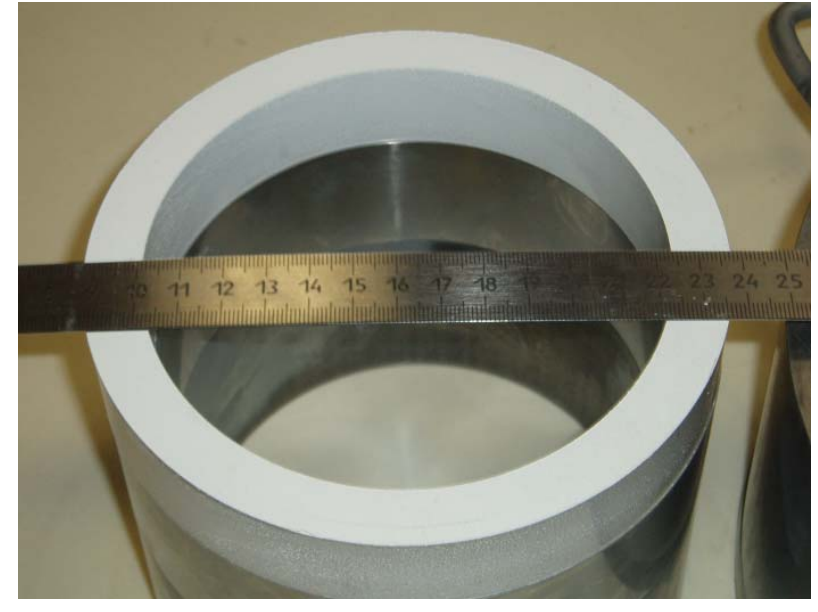

diameter $=106 \mathrm{~mm}$; thickness $=12 \mathrm{~mm}$; length $=22 \mathrm{~mm}$

$$
\begin{aligned}
& \text { width = } 6 \mathrm{~mm} \text {; } \\
& \text { height }=5 \mathrm{~mm} \text {; }
\end{aligned}
$$$$
\text { length }=\sim 108 \mathrm{~mm}
$$ 
a) Measurements of loss of ferroelectric ceramic (BSM-3):

...measuring the $\mathrm{Q}$-factor for different frequencies $(\mathrm{f})$, knowing the field distribution (from simulations), and using the law $Q \times f \approx$ const one derives the loss-tan, $\tan (\sigma) \ldots$

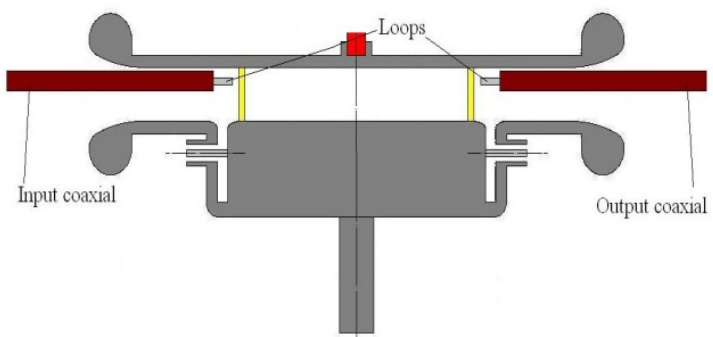

ring inside a specially designed test cavity
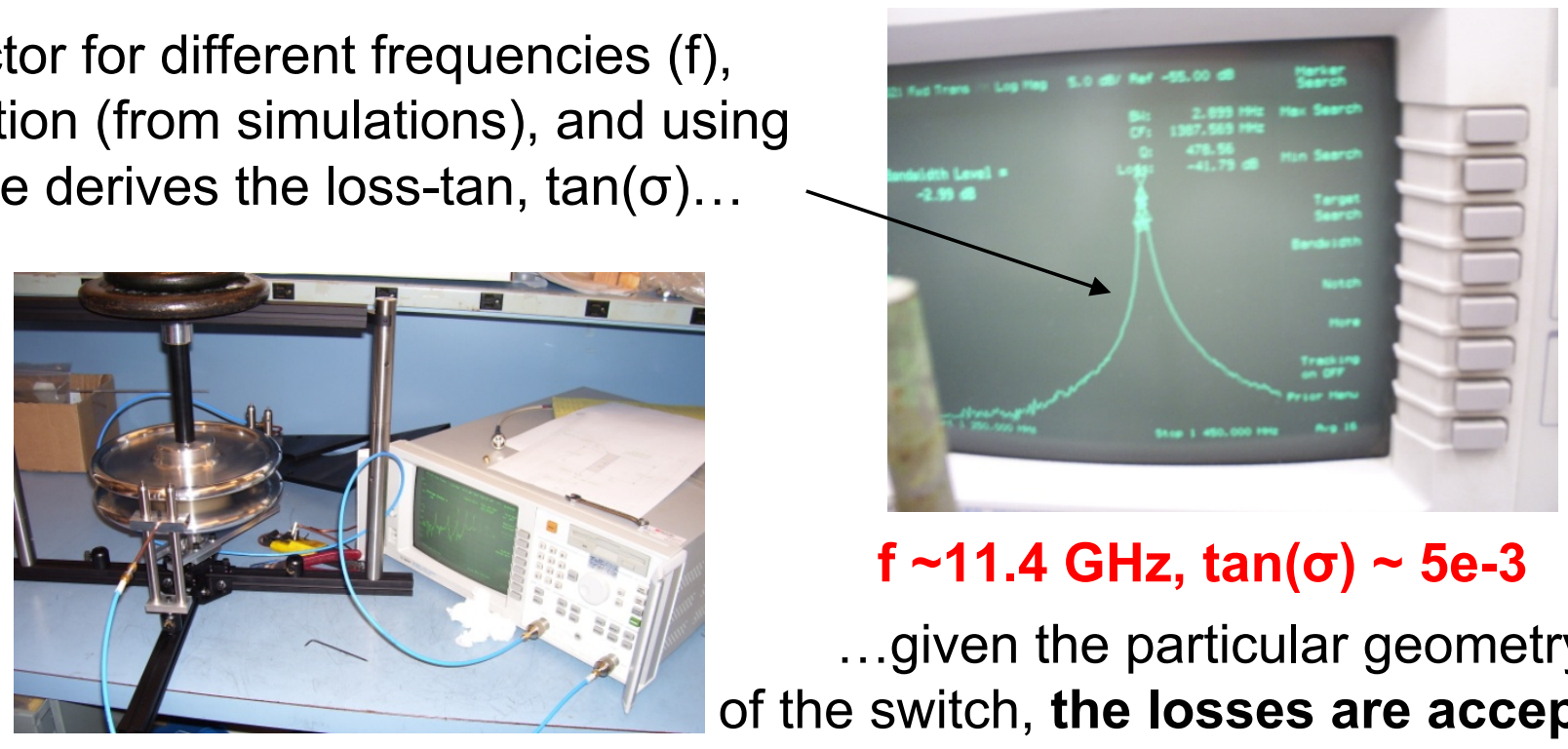

f $\sim 11.4 \mathrm{GHz}, \tan (\sigma) \sim 5 \mathrm{e}-3$

...given the particular geometry of the switch, the losses are acceptable

b) Measurements of tunability of ferroelectric material (BSM-3):
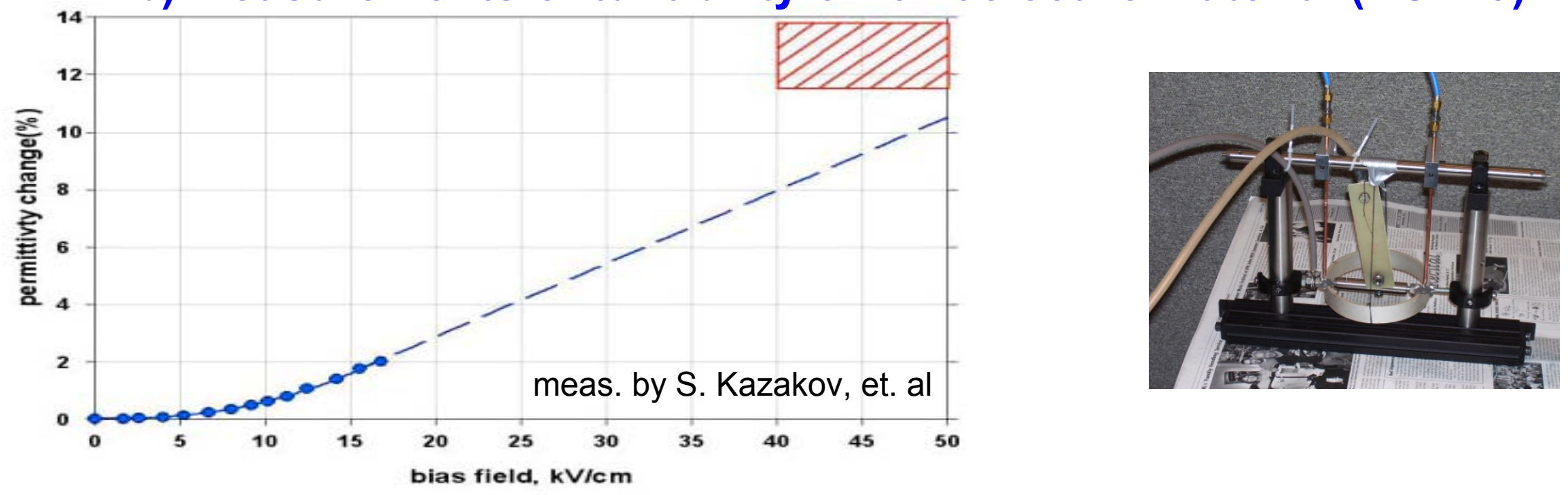

- large ring measurements 


\section{Experiment:}

X-band Switch is used in an active Compression Scheme (at NRL)
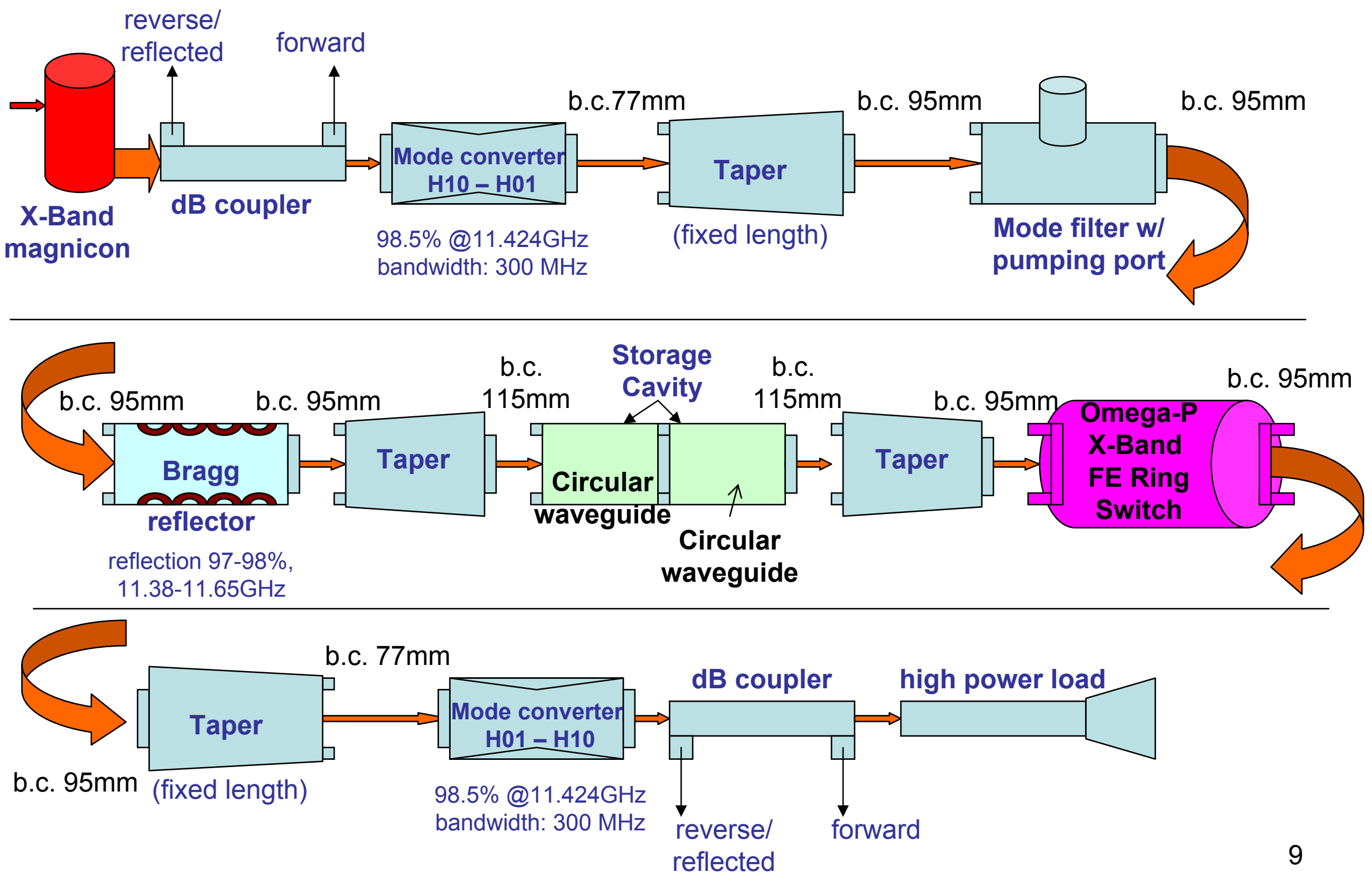


\section{Experiment:}

X-band Switch is used in an active Compression Scheme (at NRL)

...the total coupling can be changed by $8 \mathrm{~dB}$, and thus can be optimal during the filling of storage cavities, or during the energy extraction...

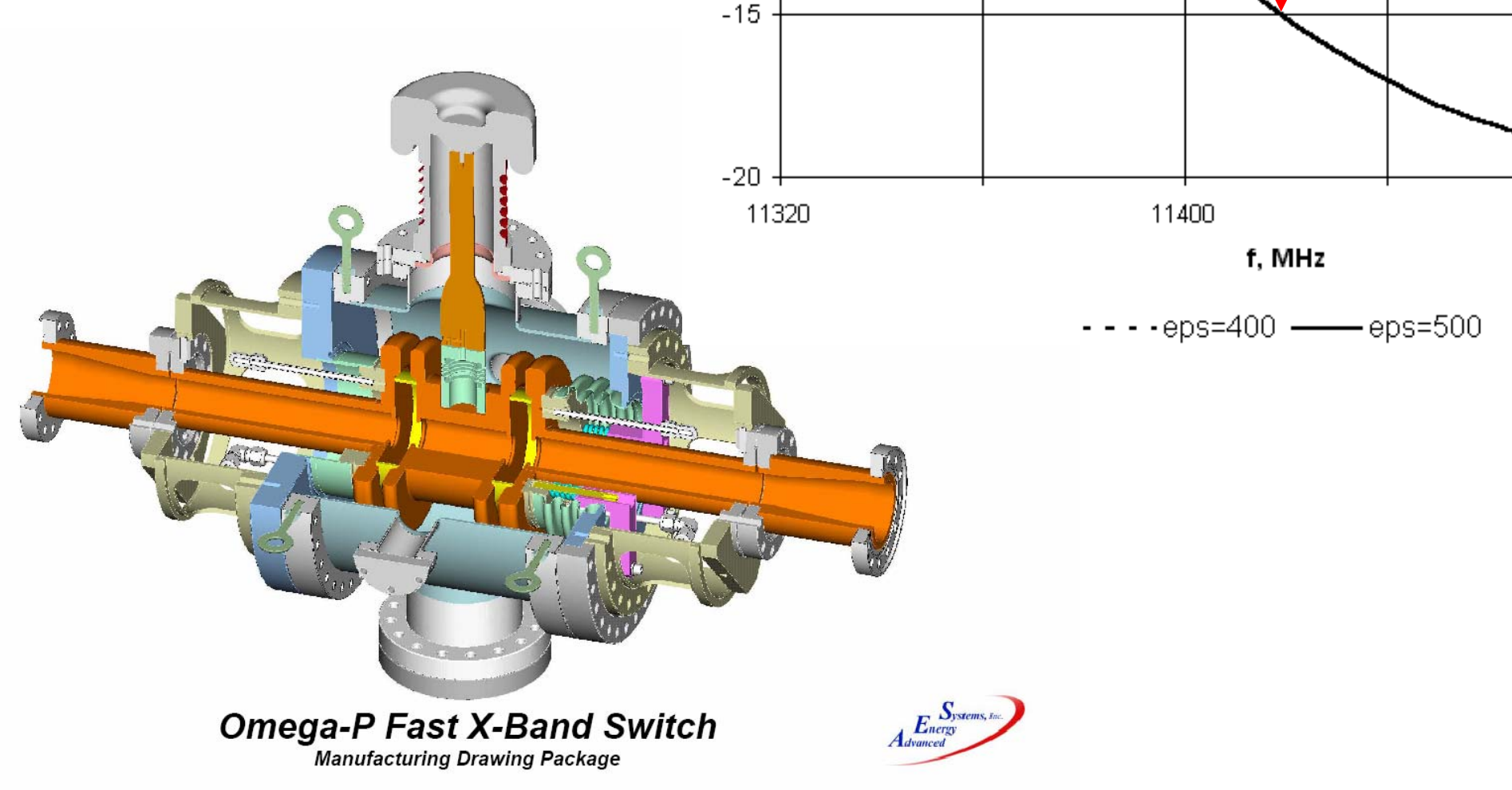


TABLE II. Parameters of $\mathrm{TE}_{031}$ switch cavity. [see parameters in Table]

\begin{tabular}{|l||l}
\hline operating frequency, GHz & 11.424 \\
\hline operating mode & $\mathrm{TE}_{031}$ \\
\hline cavity length, mm & 20 \\
\hline cavity diameter, $\mathrm{mm}$ & 118 \\
\hline coupling iris diameter, $\mathrm{mm}$ & 30 \\
\hline coupling diaphragm thickness, $\mathrm{mm}$ & 3 \\
\hline ferroelectric ring length, mm & 20 \\
\hline ferroelectric ring inner diameter, mm & 106 \\
\hline ferroelectric ring thickness, mm & 3 \\
\hline transmission in energy extraction regime $(\varepsilon=400), \mathrm{dB}$ & 0 \\
\hline transmission in energy storage regime $(\varepsilon=500), \mathrm{dB}$ & -4 \\
\hline maximum electric field in ferroelectric in energy extraction regime, $\mathrm{kV} / \mathrm{cm}$ & 20 \\
\hline maximum electric field in ferroelectric in energy storage regime, $\mathrm{kV} / \mathrm{cm}$ & 10 \\
\hline maximum electric field in vacuum in energy extraction regime, $\mathrm{kV} / \mathrm{cm}$ & 865 \\
\hline maximum electric field in vacuum in energy storage regime, $\mathrm{kV} / \mathrm{cm}$ & 675 \\
\hline
\end{tabular}

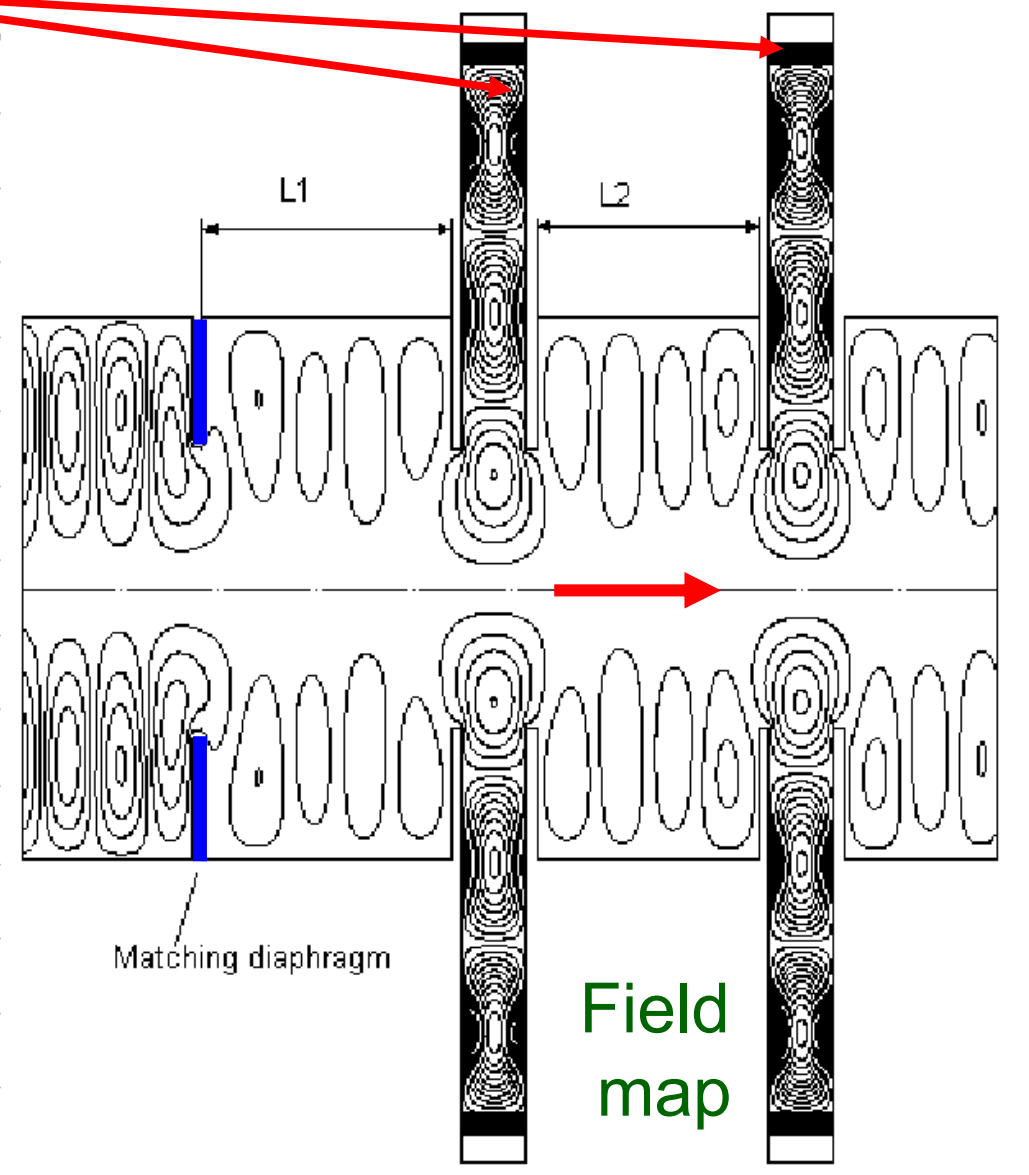

...pulse compression scheme (at Naval Research Lab) will be for operating frequency $11.43 \mathrm{GHz}$; input power $\sim 20 \mathrm{MW}$, and the input pulse duration $\sim 0.5$ micro-sec.

...the power gain, output power and output pulse duration are to be measured. 


\section{Design was developed...}

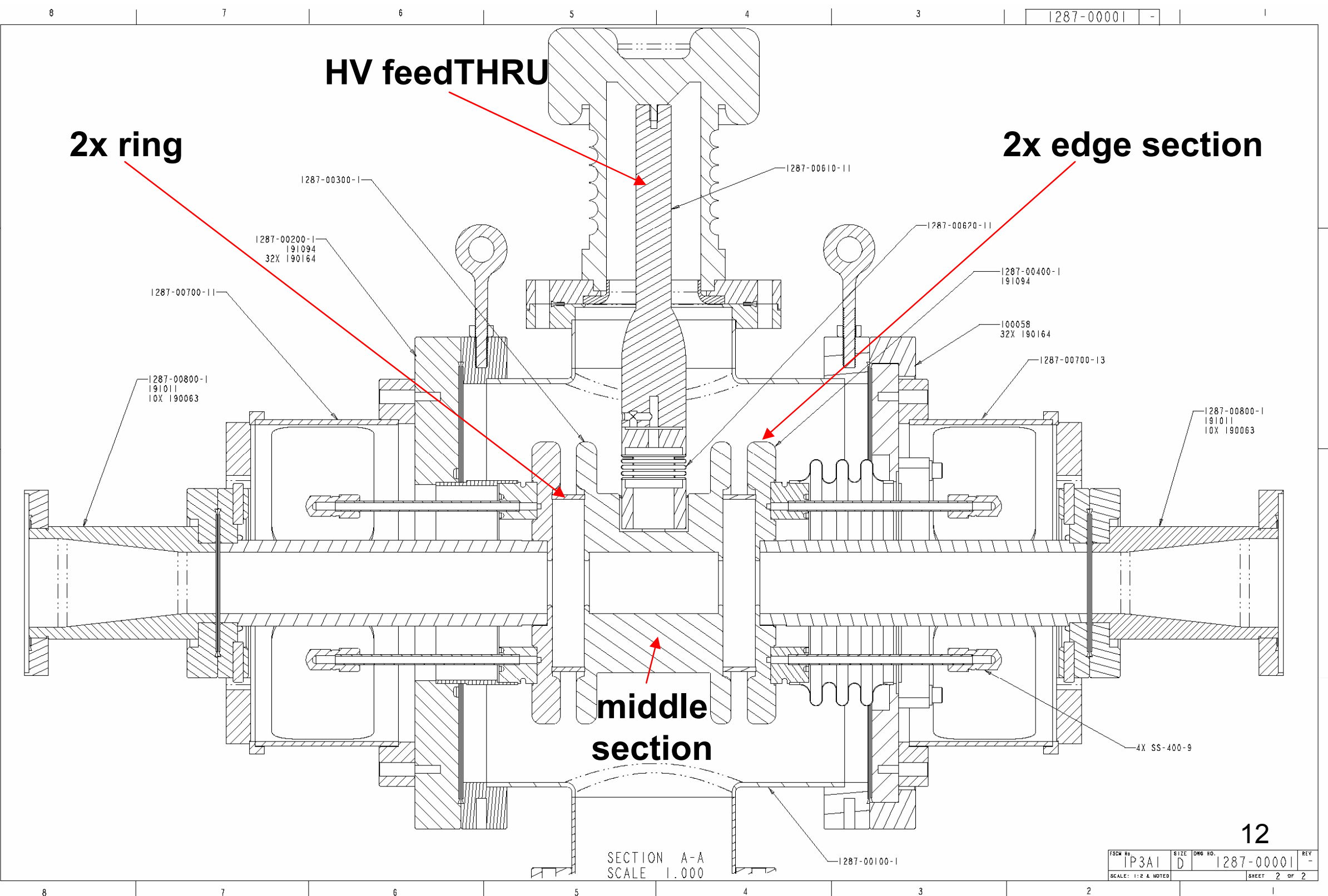


Parts were machined, the rings were manufactured...

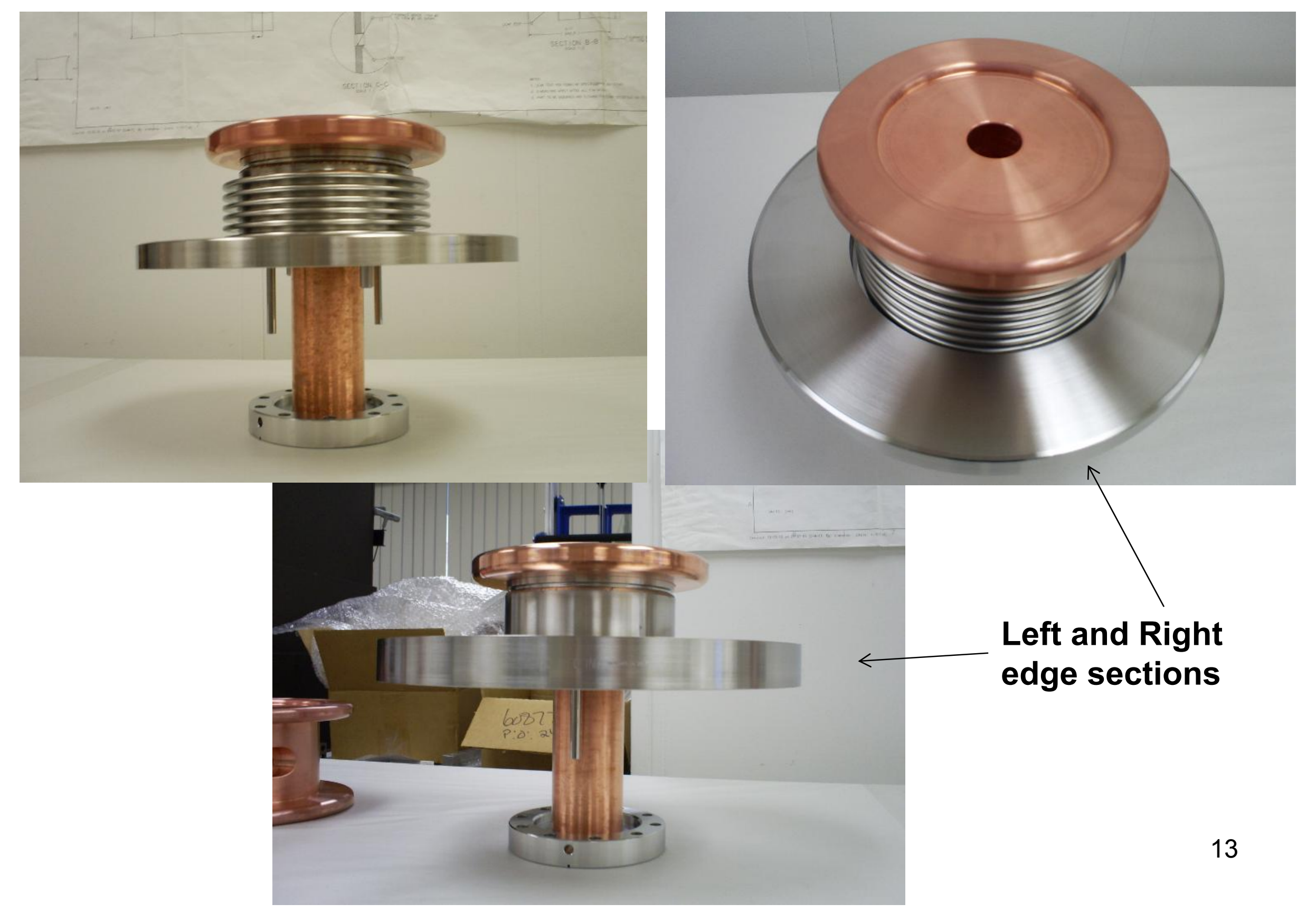




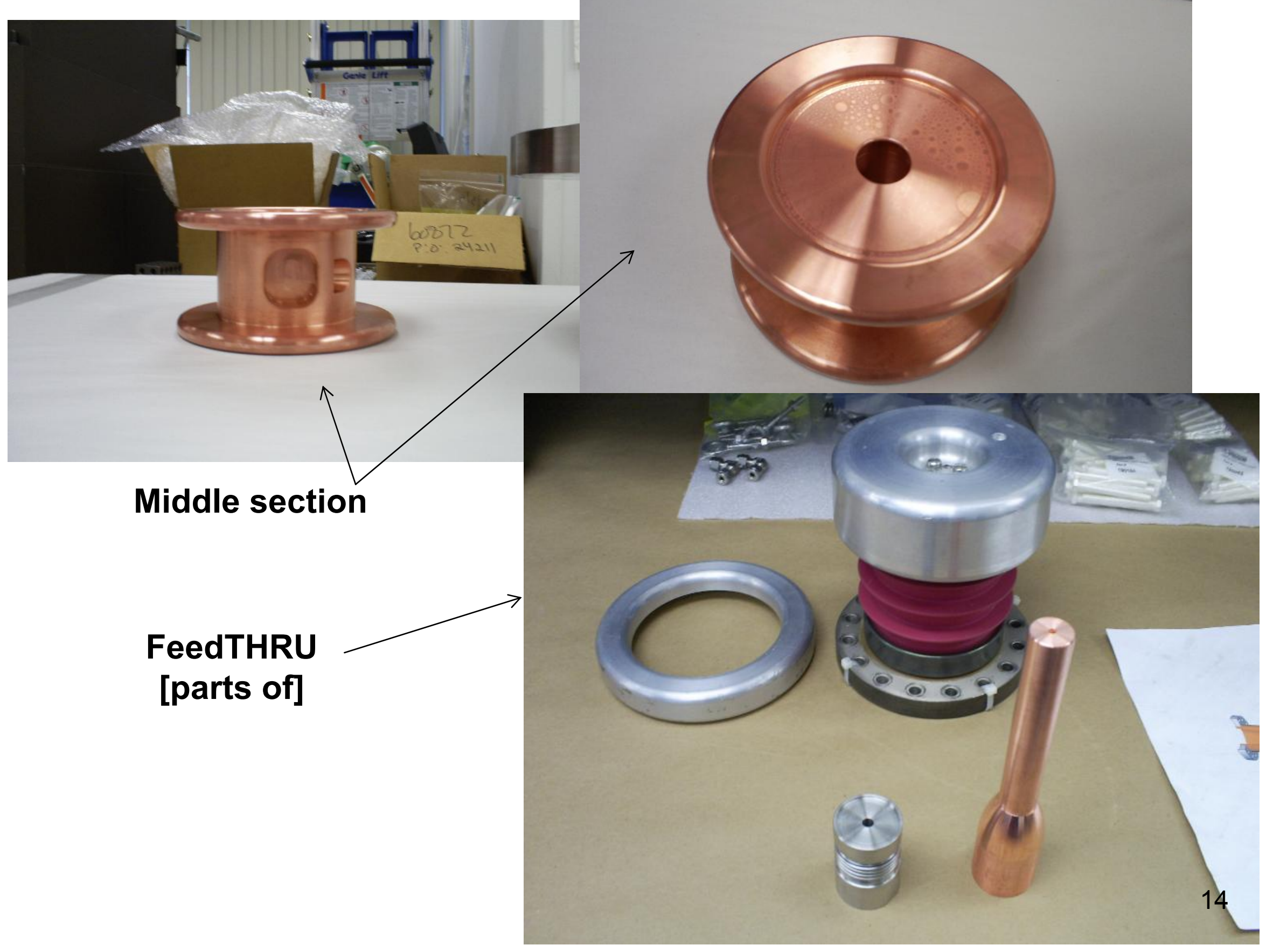




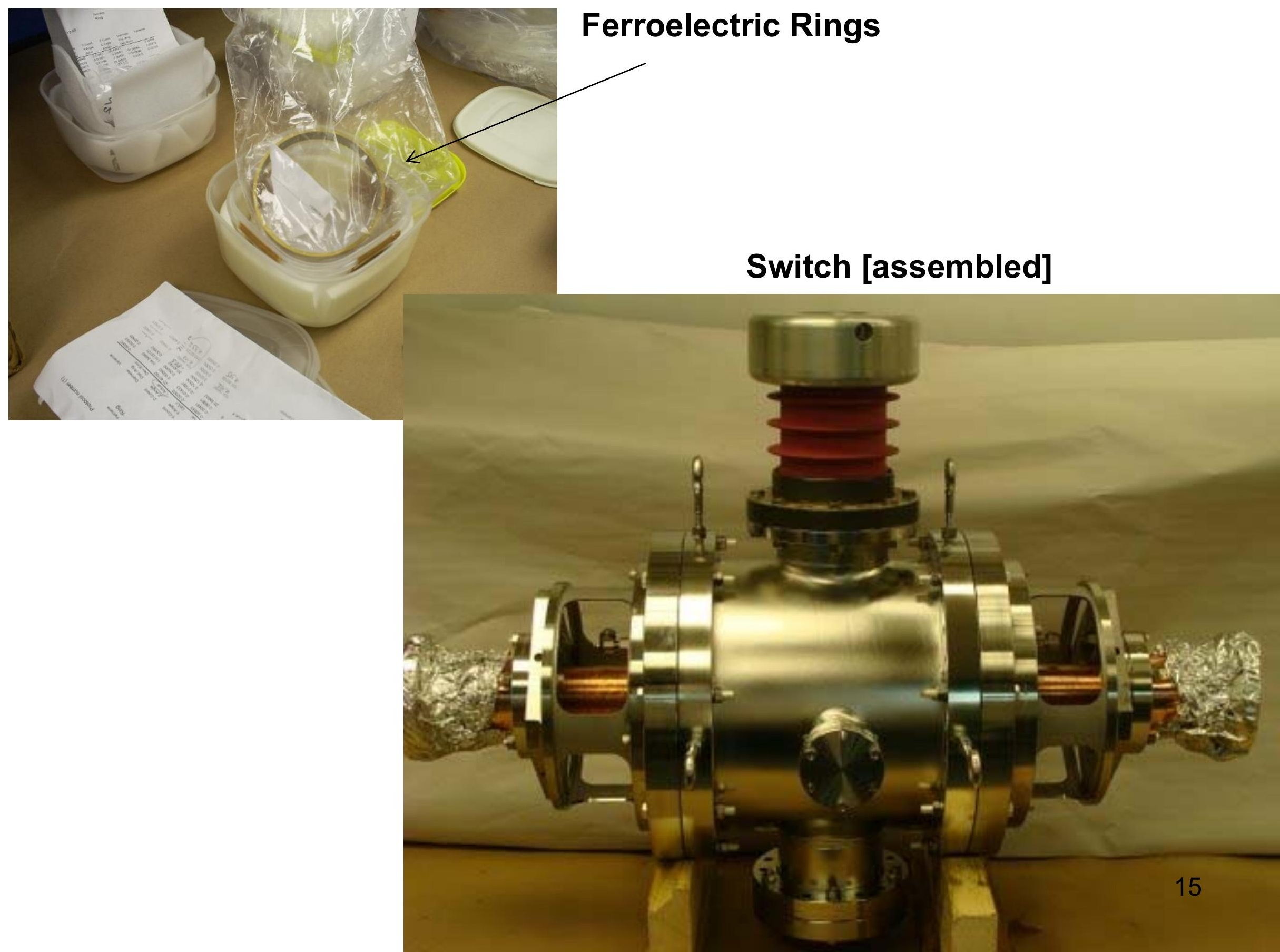




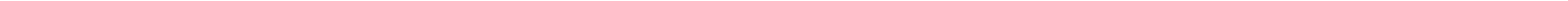


To tune the structure (by machining the grooves in designated places, several auxiliary items were made, and those of them [e.g. mode-launchers] which quire e adjusted/tuned were tuned.

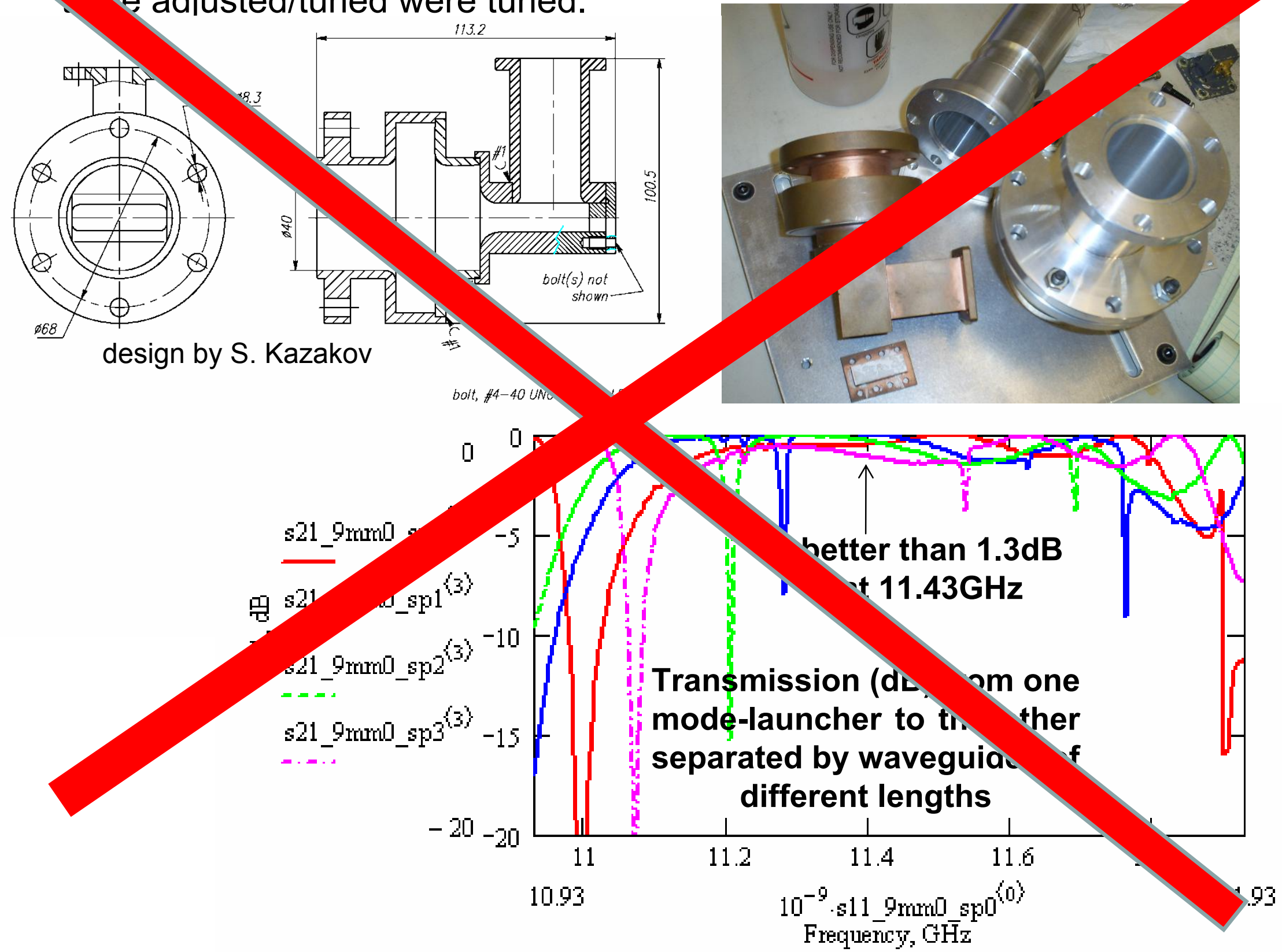




\section{Cold Measurements}




\section{Scheme to measure transmission}

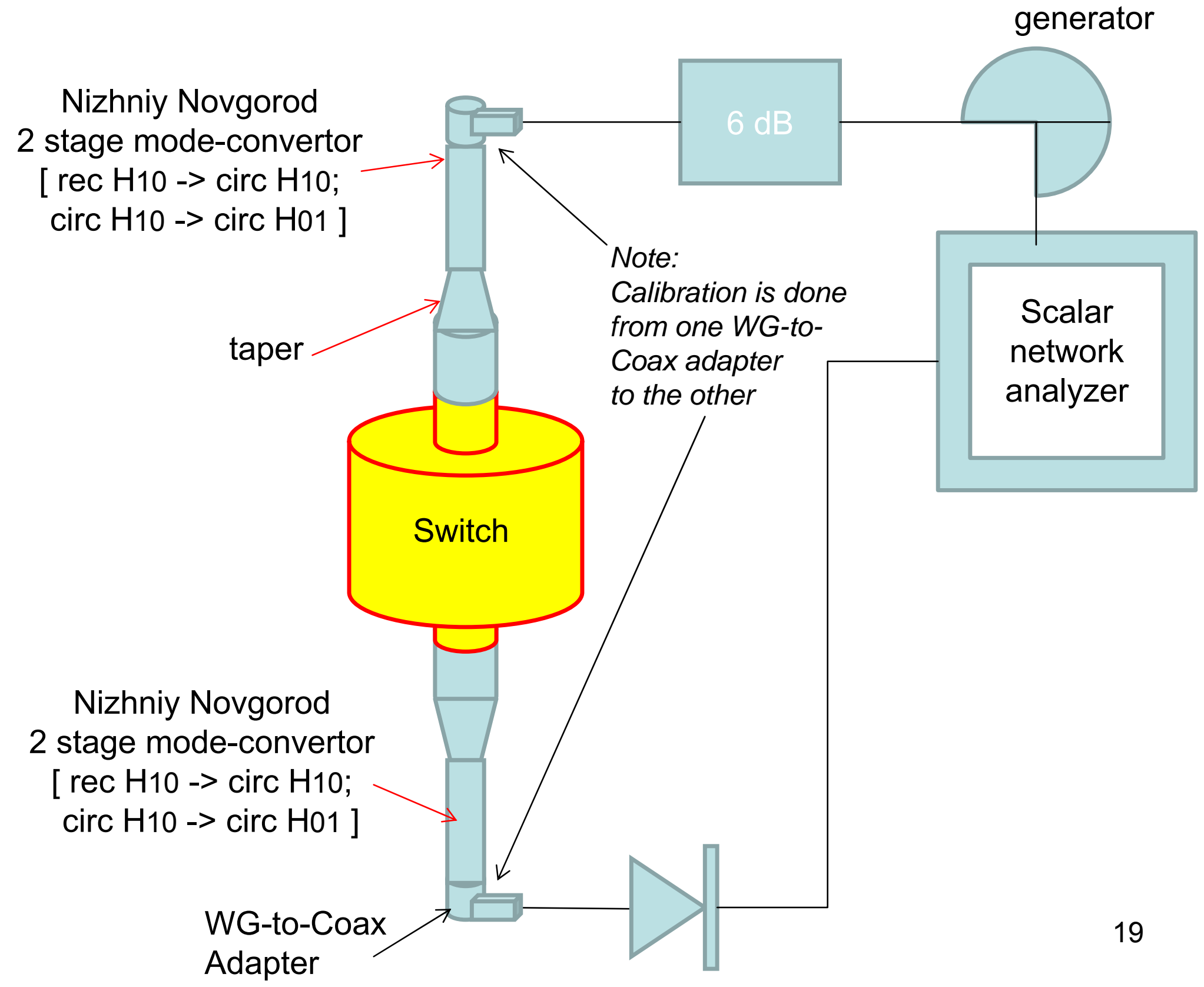




\section{Scheme to measure reflection Note:}

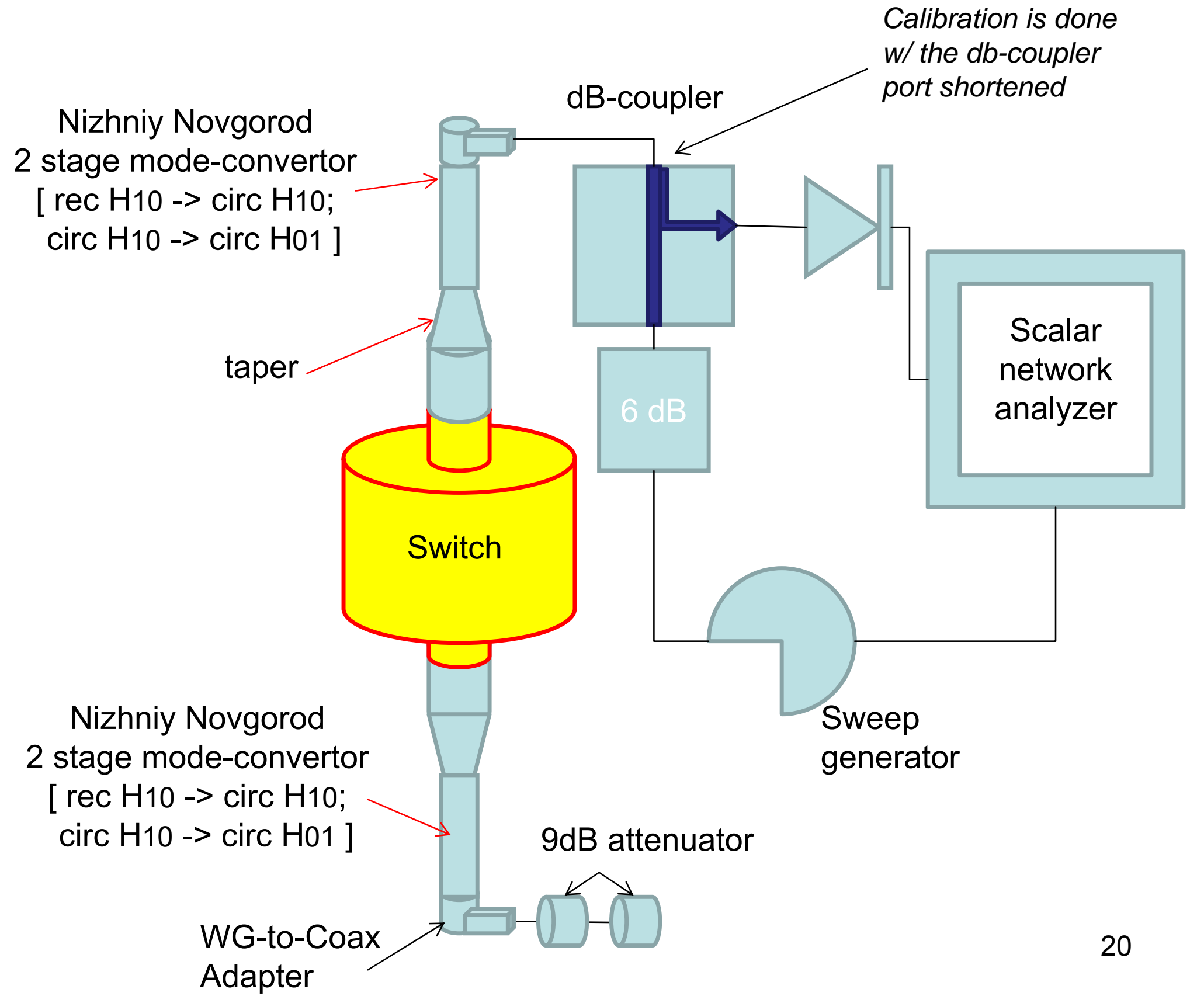



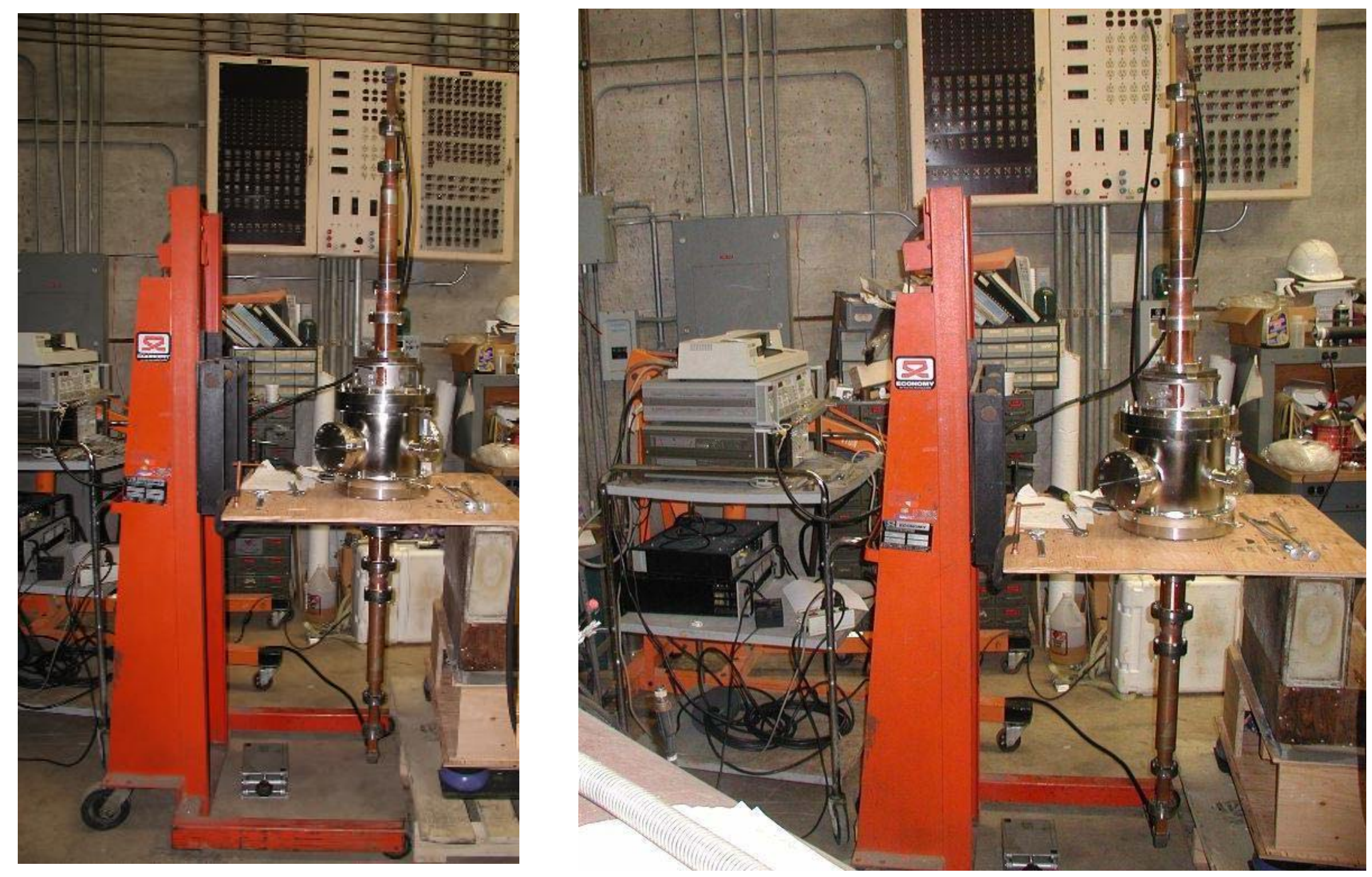


\section{Transmission with two ferroelectric rings}

1

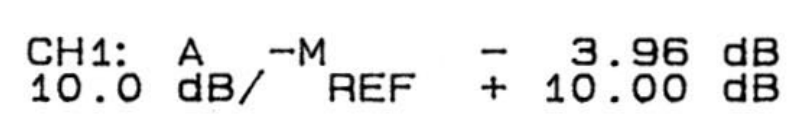
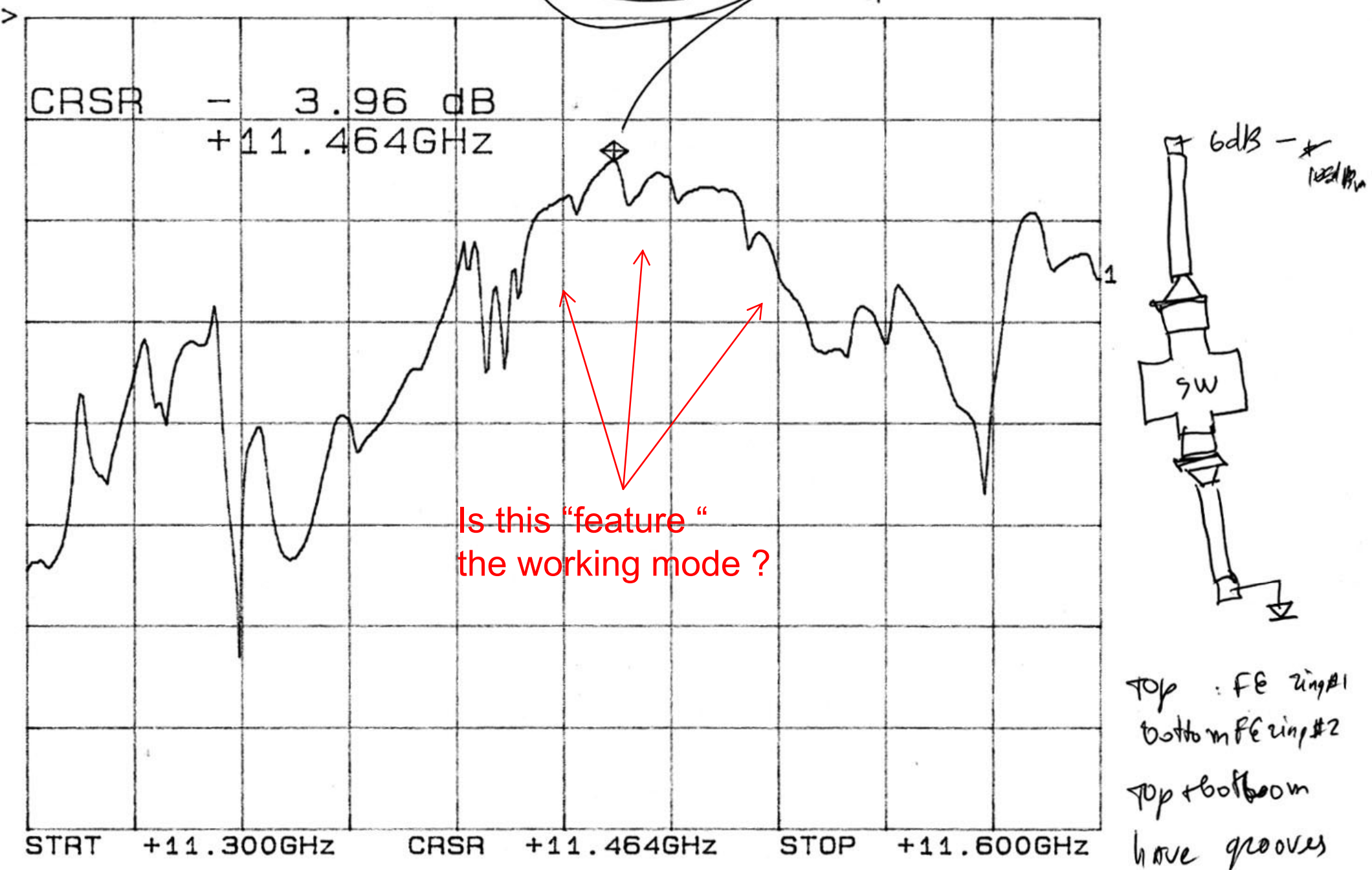

Top : FE ringt botto $m$ FG rimp $\$ 2$

Top tbotboom

have qrooves $0.375 \times \frac{31}{1000} 8 p$ 
Reflection with two ferroelectric rings $\left(\begin{array}{l}1 \\ 0\end{array}\right)$ Ueflection

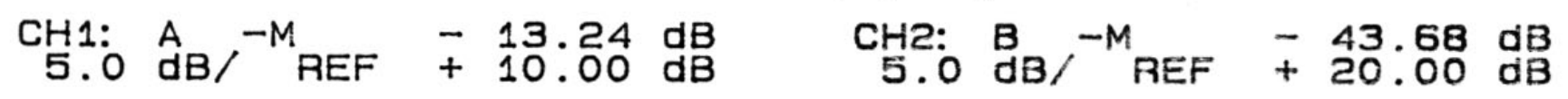

12

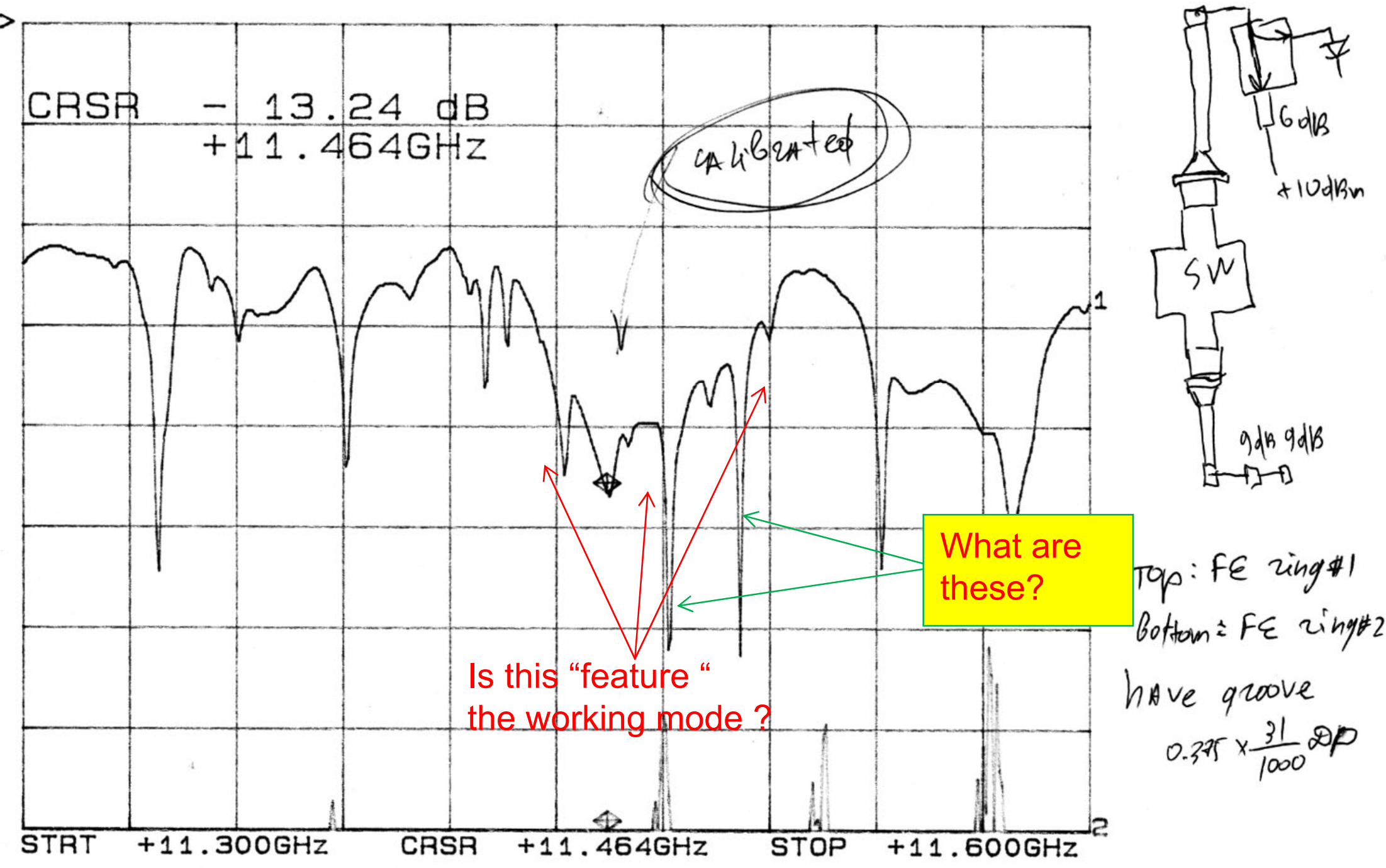


Transmission with two aluminum "dummy" rings

10:0 $\mathrm{dB}^{\mathrm{A}} /^{-M_{\text {REF }}} \mp 20.00 \mathrm{~dB}$

1
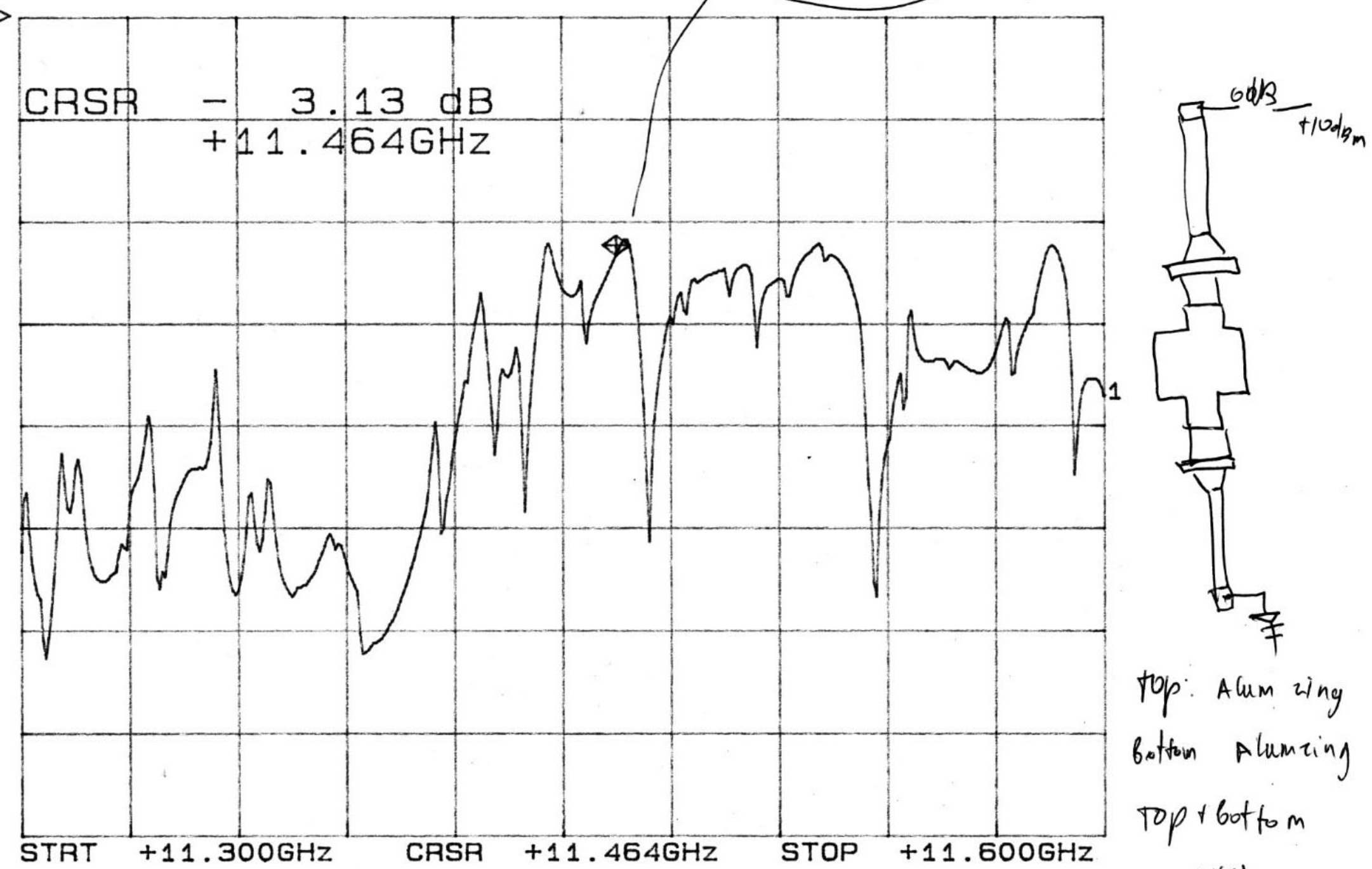

top: Alum wing

bottom Alumeing

Topt bottom

g cooves

$0,275 \times \frac{11}{1000} 29$ 
Reflection with two aluminum "dummy" rings $\left(\begin{array}{l}1 \\ 0\end{array}\right)$ refection

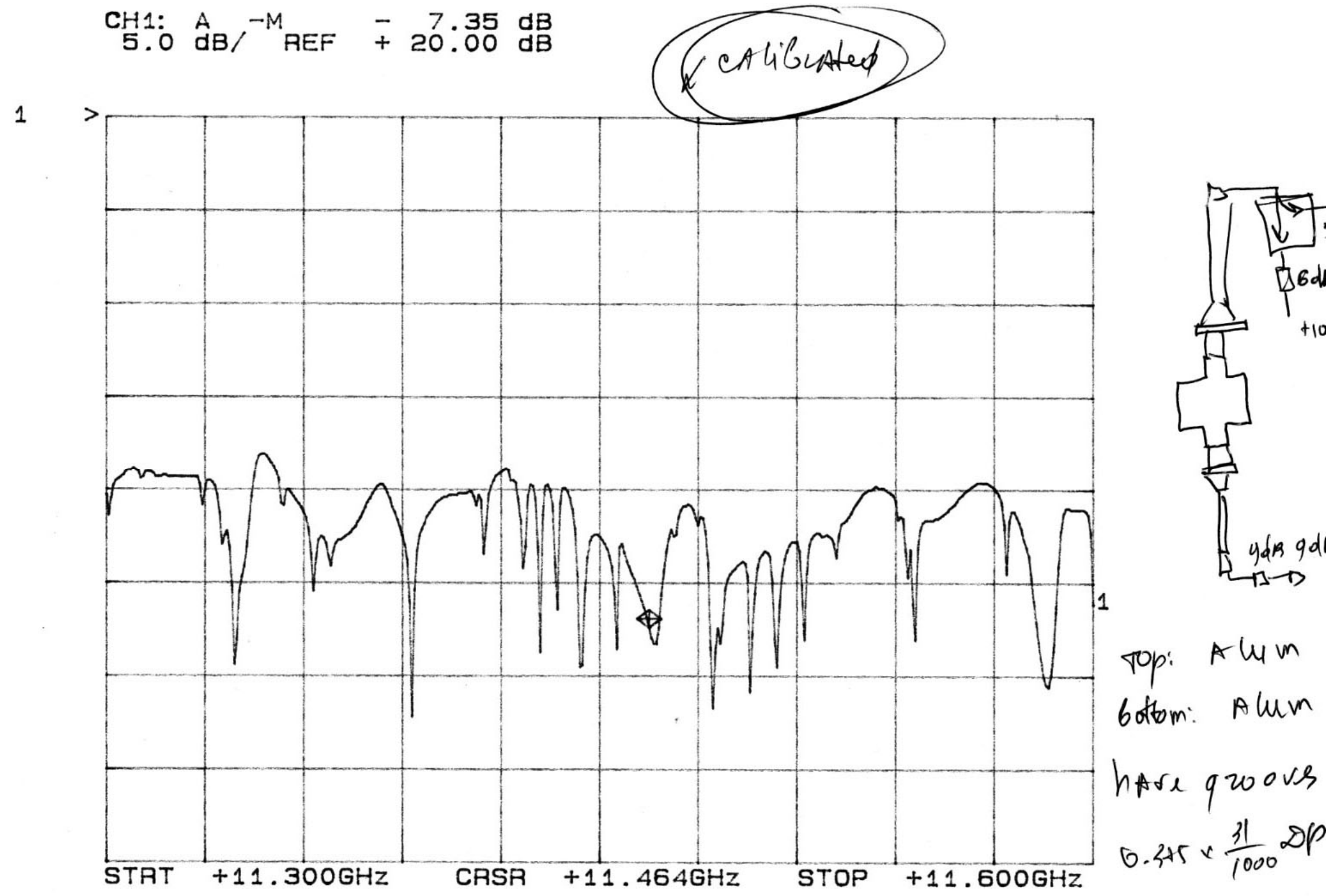


If one compares the sum of the transmission $(T)+$ reflection $(R)$ when the two FE rings are installed with the sum of the transmission + reflection when two aluminum rings are installed at the frequency $11,464 \mathrm{GHz}$, one gets:

$\mathrm{T}+\mathrm{R}=45 \%$

w/ FE rings

$\mathrm{T}+\mathrm{R}=65 \%$

$\mathrm{w} /$ aluminum rings

The difference is $20 \%$.

$\ldots$ thus, there is at least $1 \mathrm{~dB}$ loss in the ferroelectric rings

... what to do? 


\section{Conclusions}

- Designs for X-band phase shifter and X-band switch were presented

- X-band switch is simulated, designed, and its parts are manufactured

- Ceramic rings are manufactured

- X-band switch is assembled and vacuum checked

- Switch must be still tuned to frequency

- Auxiliary items

- are manufactured

- items are tuned to operate at $\sim 11.4 \mathrm{GHz}$

- X-band switch test

- Switch is shipped to NRL

- It must be still installed to the circuitry, and operated... 
Cannot simulate full 360 geometries:

* out of memory is thrown when simulating geometries $\mathrm{w} / \mathrm{FE}$-rings

* long commutation time (3-4 days) w/ poor frequency convergence ( $10-20 \%$ only) when simulating aluminum rings

Since we have no access to advanced computers (like those "Avatar guys")...
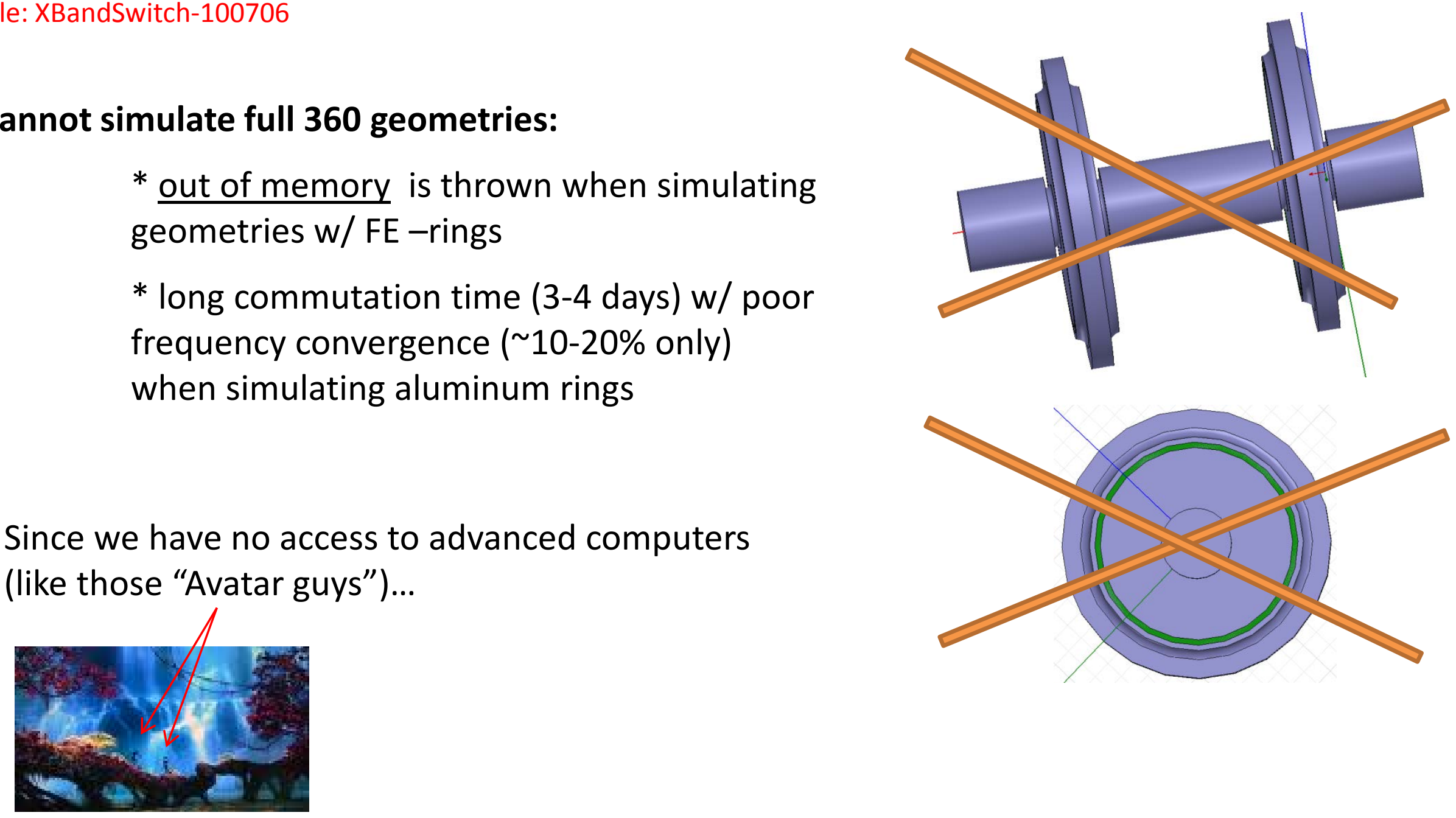

... we are using a sector like geometry good to capture the axis-symmetric (axis-sym) modes;

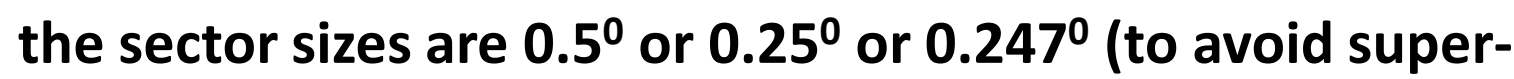
periodic modes) and deliver excellent convergence 


\section{Chapter 1: Spectrum}

(no grooves) 
(axis-sym) FE modes w/ the Q-factor 166- 167

(each exists basically inside one of the FE-rings)

\begin{tabular}{|l|l|l|l|l|l|}
\hline $\begin{array}{l}\text { Mode } \\
\text { F,MHz }\end{array}$ & $\begin{array}{l}2 \text { cav } \\
\text { model }\end{array}$ & $\begin{array}{l}\text { E-sym } \\
\text { model }\end{array}$ & $\begin{array}{l}\text { H-sym } \\
\text { model }\end{array}$ & $\begin{array}{l}\text { Single } \\
\text { cav }\end{array}$ \\
\hline 11034 & Ns & Ns & Ns & 1 \\
\hline 11066.7 & 2 & Ns & Ns & 1 \\
\hline 11173.5 & 2 & Ns & Ns & 1 \\
\hline 11296 & 2 & 1 & 1 & 1 \\
\hline 11325.4 & 2 & 1 & 1 & 1 \\
\hline 11337 & 2 & 1 & 1 & 1 \\
\hline 11356.8 & 2 & 1 & 1 & 1 \\
\hline 11379.6 & 2 & 1 & 1 & 1 \\
\hline 11547 & 2 & 1 & 1 & 1 \\
\hline 11587.4 & 2 & 1 & 1 & 1 \\
\hline 11626.6 & Ns & Ns & Ns & 1 \\
\hline 11642 & Ns & Ns & Ns & 1 \\
\hline Ns = not simulated & & & & \\
\hline
\end{tabular}

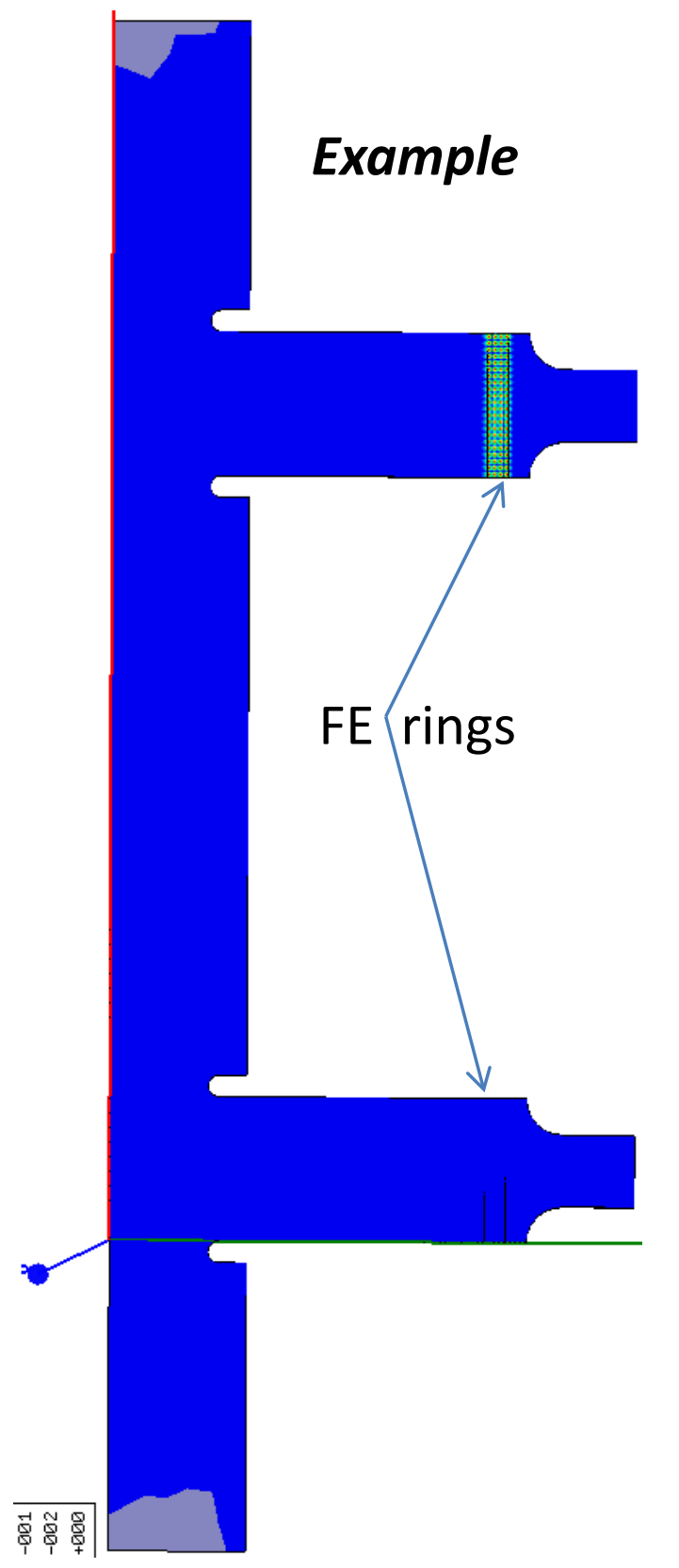


(axis-sym) Phantom mode w/ the Q-factor $~ 7.9$ (in both cases: with FE rings or with Alum rings)

with FE rings

with Alum rings

\begin{tabular}{|l|l|l|l|l|}
\hline $\begin{array}{l}2 \text { cav } \\
\text { model }\end{array}$ & $\begin{array}{l}\text { E-sym } \\
\text { model }\end{array}$ & $\begin{array}{l}\text { H-sym } \\
\text { model }\end{array}$ & $\begin{array}{l}\text { Single } \\
\text { cav }\end{array}$ & $\begin{array}{l}2 \text { cav } \\
\text { model }\end{array}$ \\
\hline absent & 11281.4 & 11281.4 & 11264 & $\begin{array}{c}2 \text { modes } \\
@ \\
11282.6\end{array}$ \\
\hline
\end{tabular}




\section{Working Mode(s)}

(in both cases: with FE rings or with Alum rings)

with FE rings

\begin{tabular}{|c|c|c|c|c|c|}
\multicolumn{5}{|c|}{ rings } \\
\hline & $\begin{array}{l}2 \text { cav } \\
\text { model }\end{array}$ & $\begin{array}{l}\text { E-sym } \\
\text { model }\end{array}$ & $\begin{array}{l}\text { H-sym } \\
\text { model }\end{array}$ & $\begin{array}{l}\text { Single } \\
\text { cav }\end{array}$ & $\begin{array}{l}2 \text { cav } \\
\text { model }\end{array}$ \\
\hline $\begin{array}{c}\mathrm{F}, \mathrm{MHz} \\
(\mathrm{Q})\end{array}$ & $\begin{array}{c}11483.5 \\
(263)\end{array}$ & $\begin{array}{c}11483.5 \\
(263)\end{array}$ & & & $\begin{array}{c}11510.3 \\
(288.7)\end{array}$ \\
\hline $\begin{array}{c}\mathrm{F}, \mathrm{MHz} \\
(\mathrm{Q})\end{array}$ & & & & $\begin{array}{c}11526.3 \\
(97.7)\end{array}$ \\
\hline $\begin{array}{c}\mathrm{F}, \mathrm{MHz} \\
(\mathrm{Q})\end{array}$ & $\begin{array}{c}11542.5 \\
(186)\end{array}$ & & $\begin{array}{c}11542.5 \\
(186)\end{array}$ & & $\begin{array}{c}11579.5 \\
(187.8)\end{array}$ \\
\hline
\end{tabular}

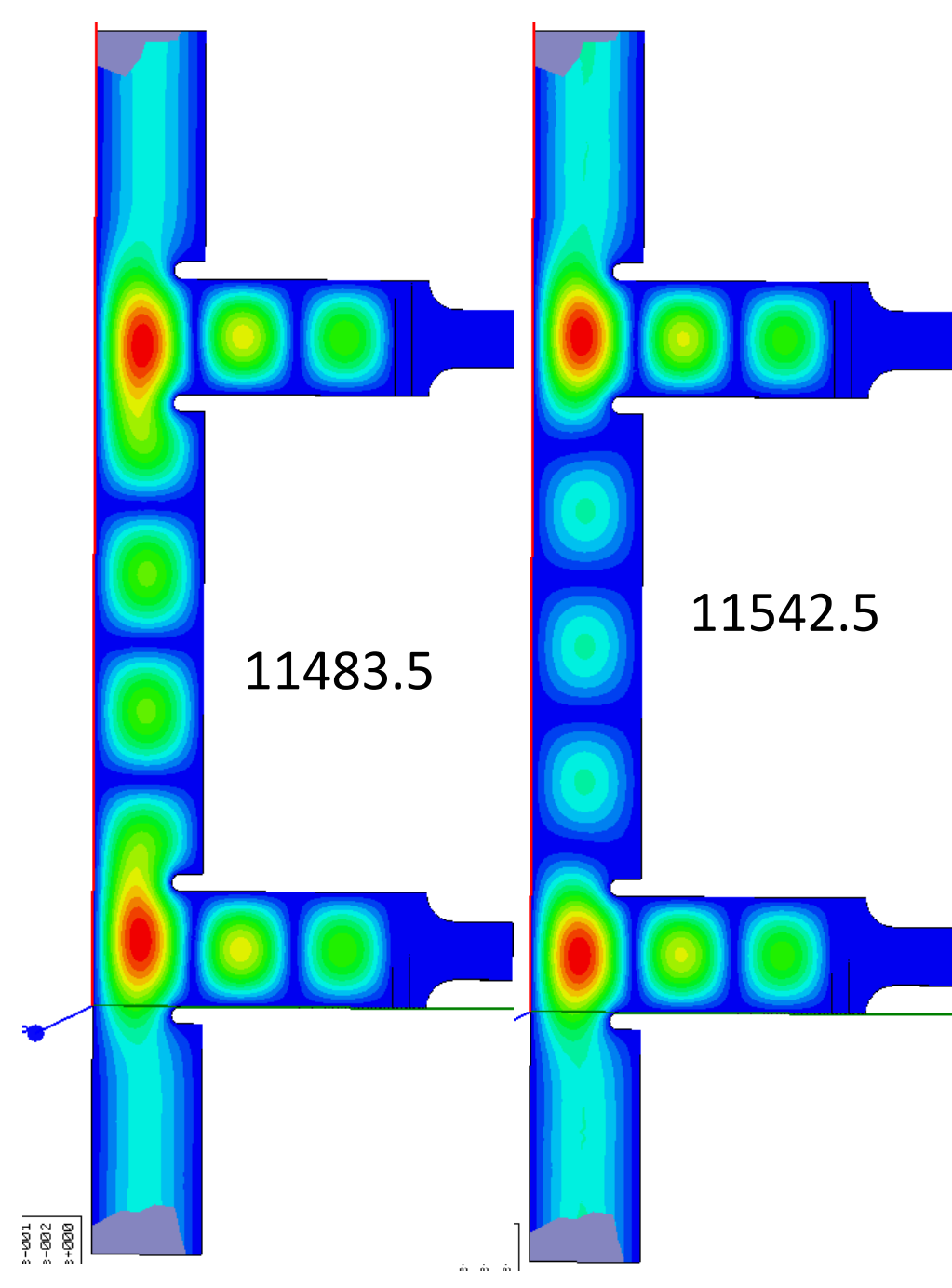


(axis-sym) Modes in the Switch w/ alum rings

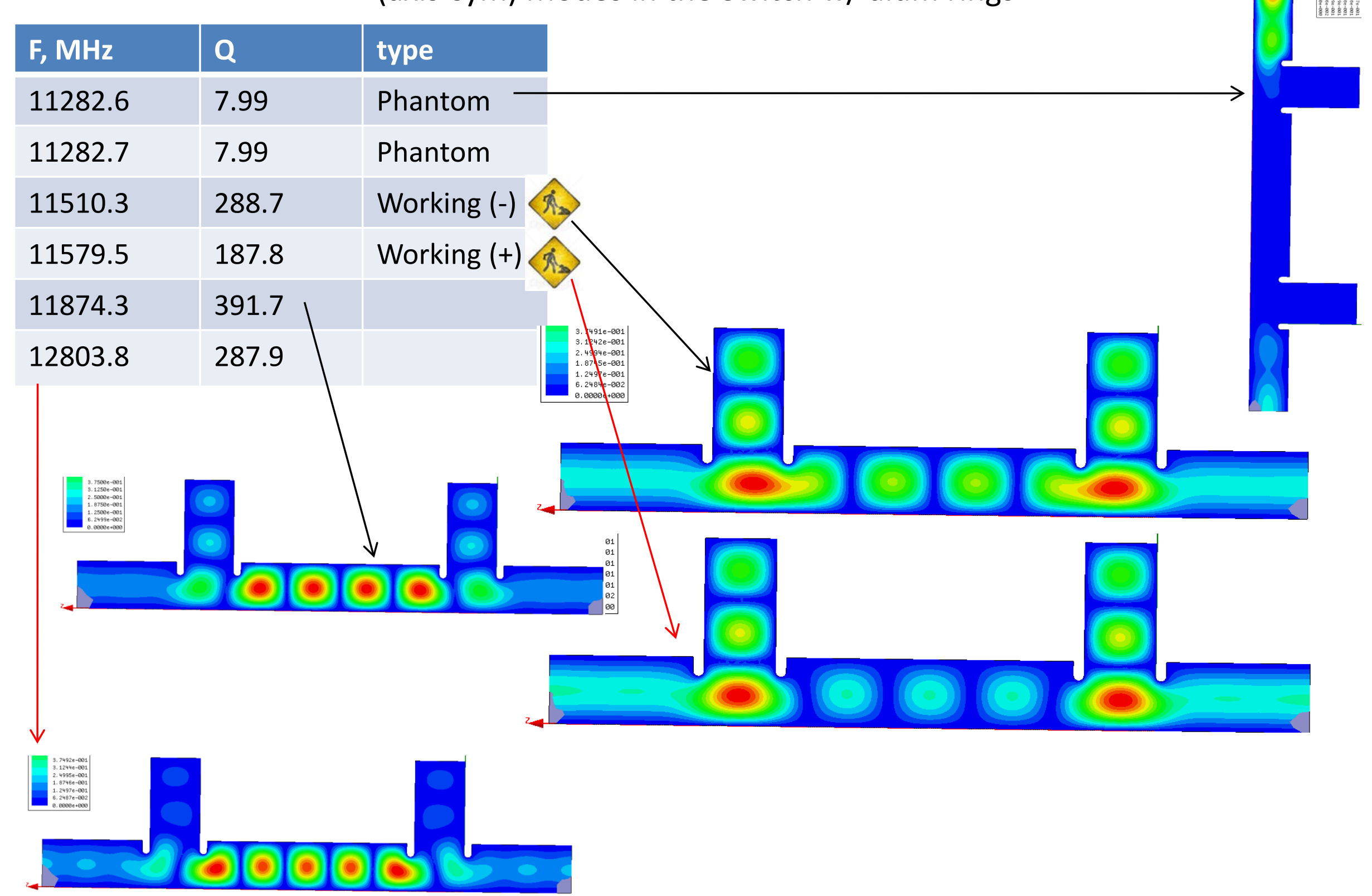




\section{Epsilon \\ Chapter 2:}

changes

\section{Transmission/}

Reflection

of the Switch

w/ FE Rings

(no grooves) 


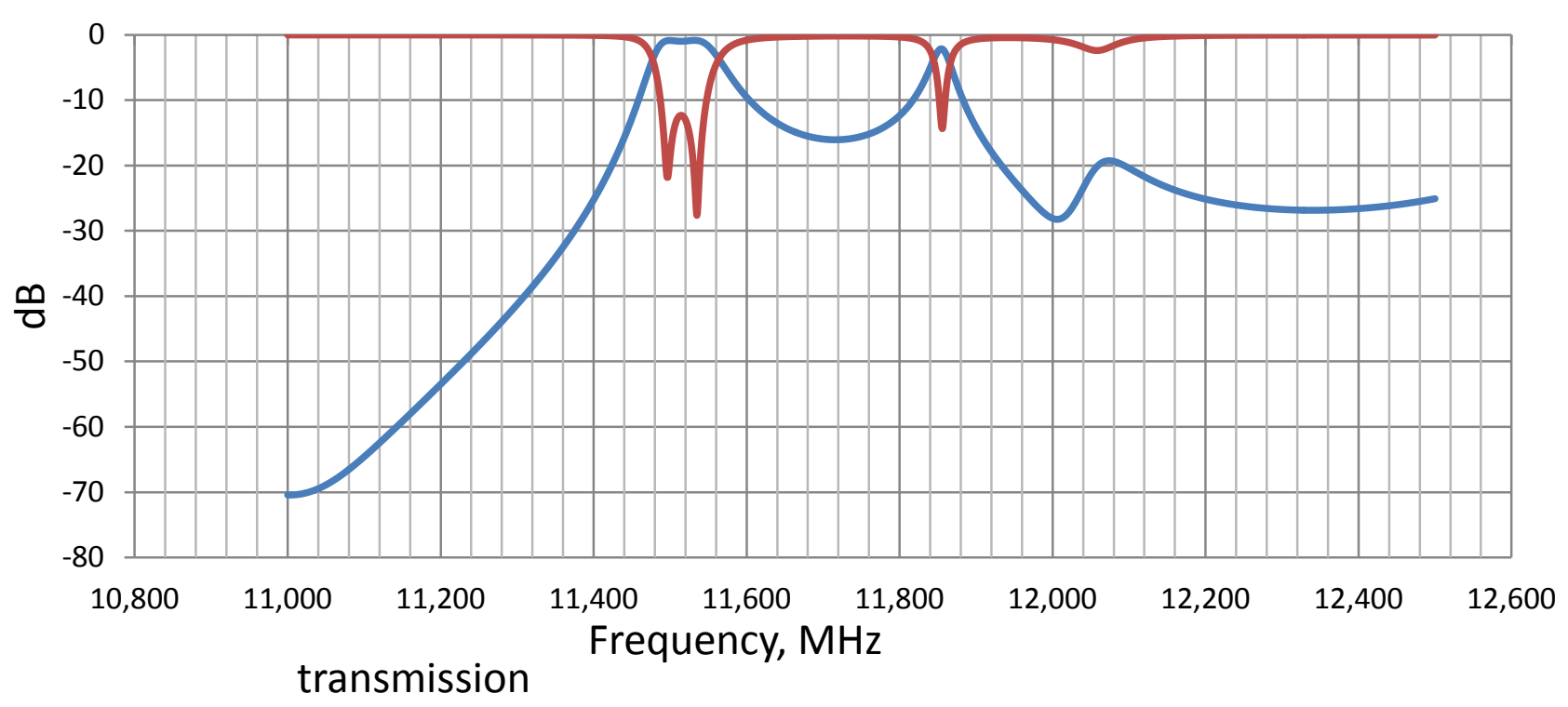

FE rings, no grooves

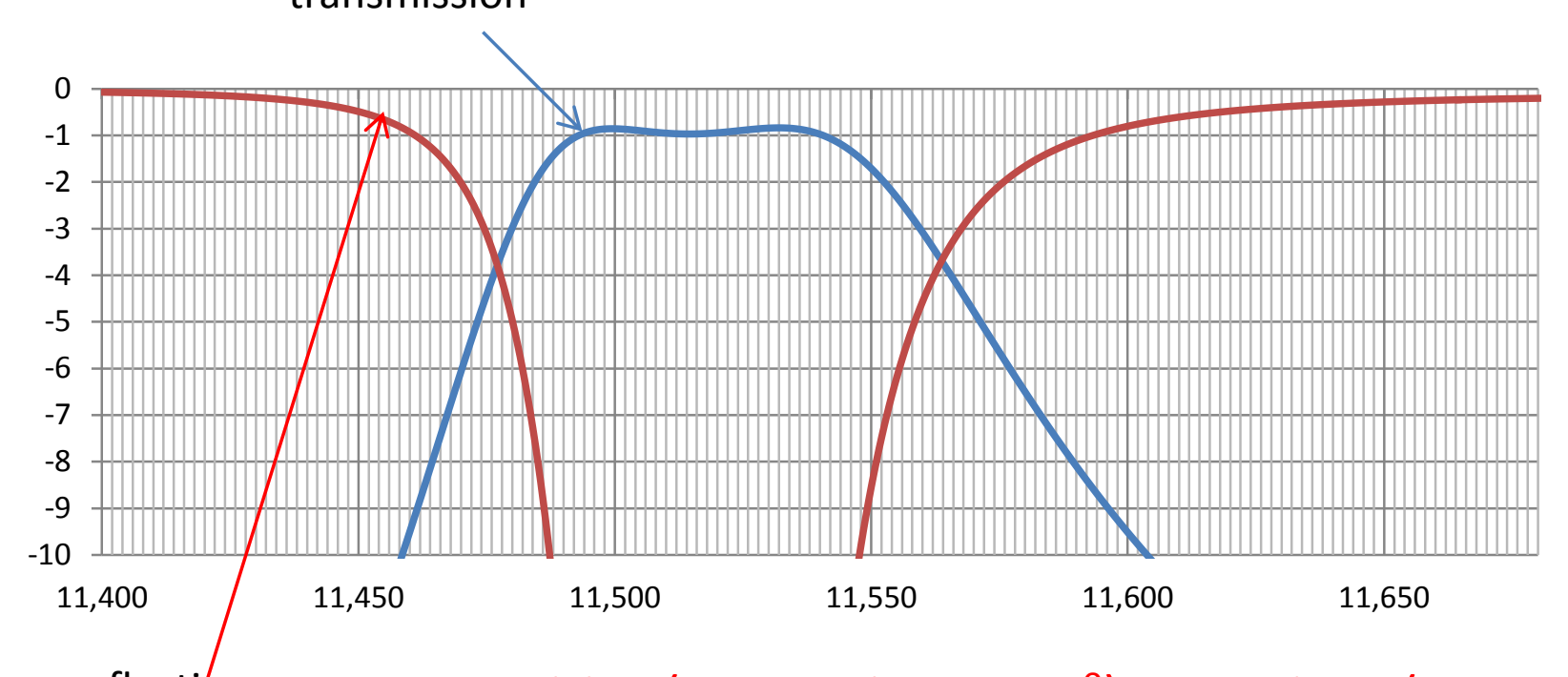

reflection

2 cavities (sector size $0.247^{\circ}$ ); 2 FE-rings (one per cavity);

Dielectric constant 460; Loss-tan .006;

Central frequency (CF) 11,516 GHz; Attenuation (@ CF) -1dB;

Bandwidth @-3dB level below the CF level (BW) $85 \mathrm{MHz}$;

Right edge frequency corresponding to $-6 \mathrm{~dB}$ (REF) $11,577 \mathrm{MHz}$ 
FE rings,

no grooves

epsilon changes

loss-tan .006
Changing eps to $400-370$ delivers the increment in transmission 6.25 - 6.55;

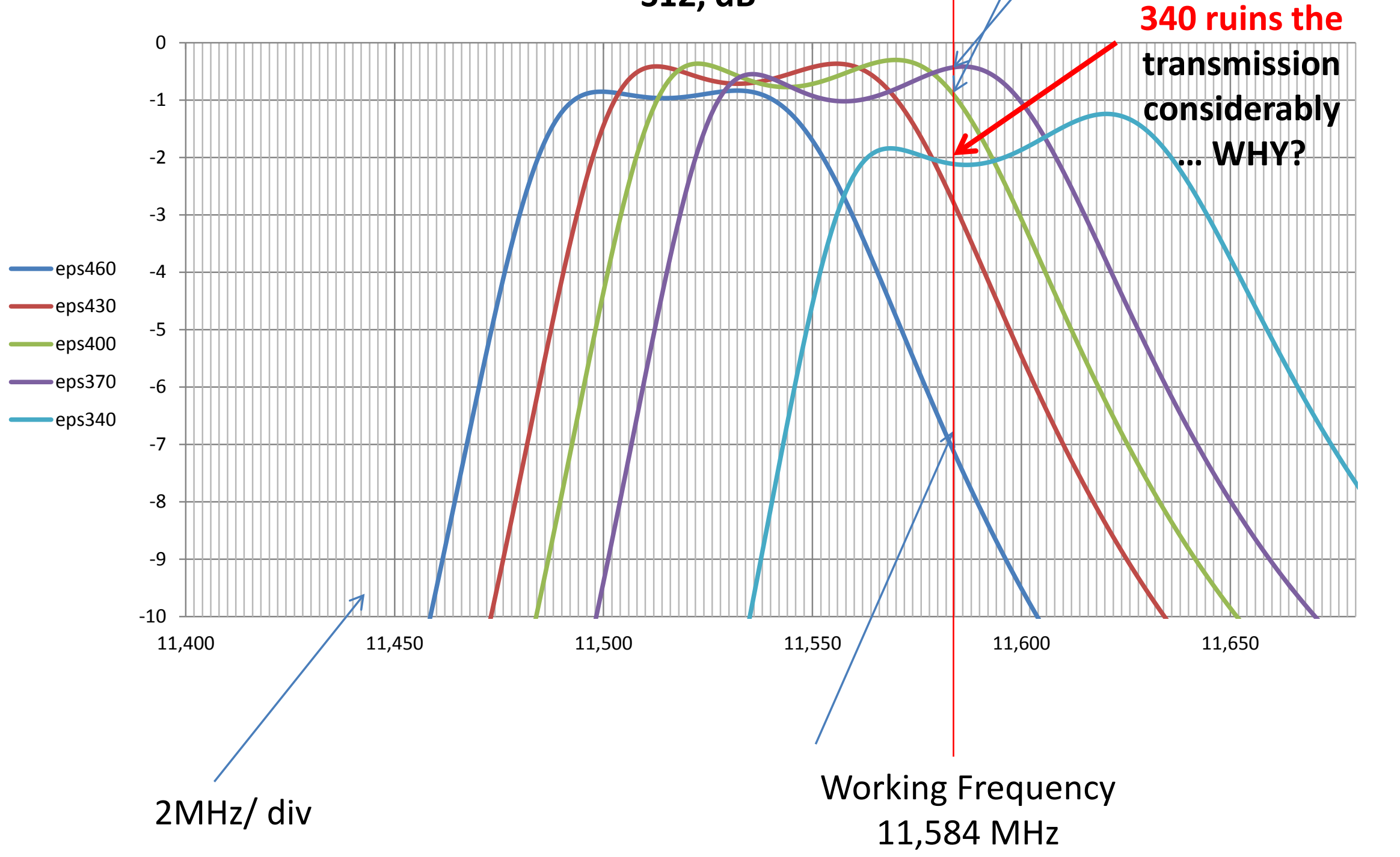




\section{FE rings,}

no grooves

epsilon changes

loss-tan .006

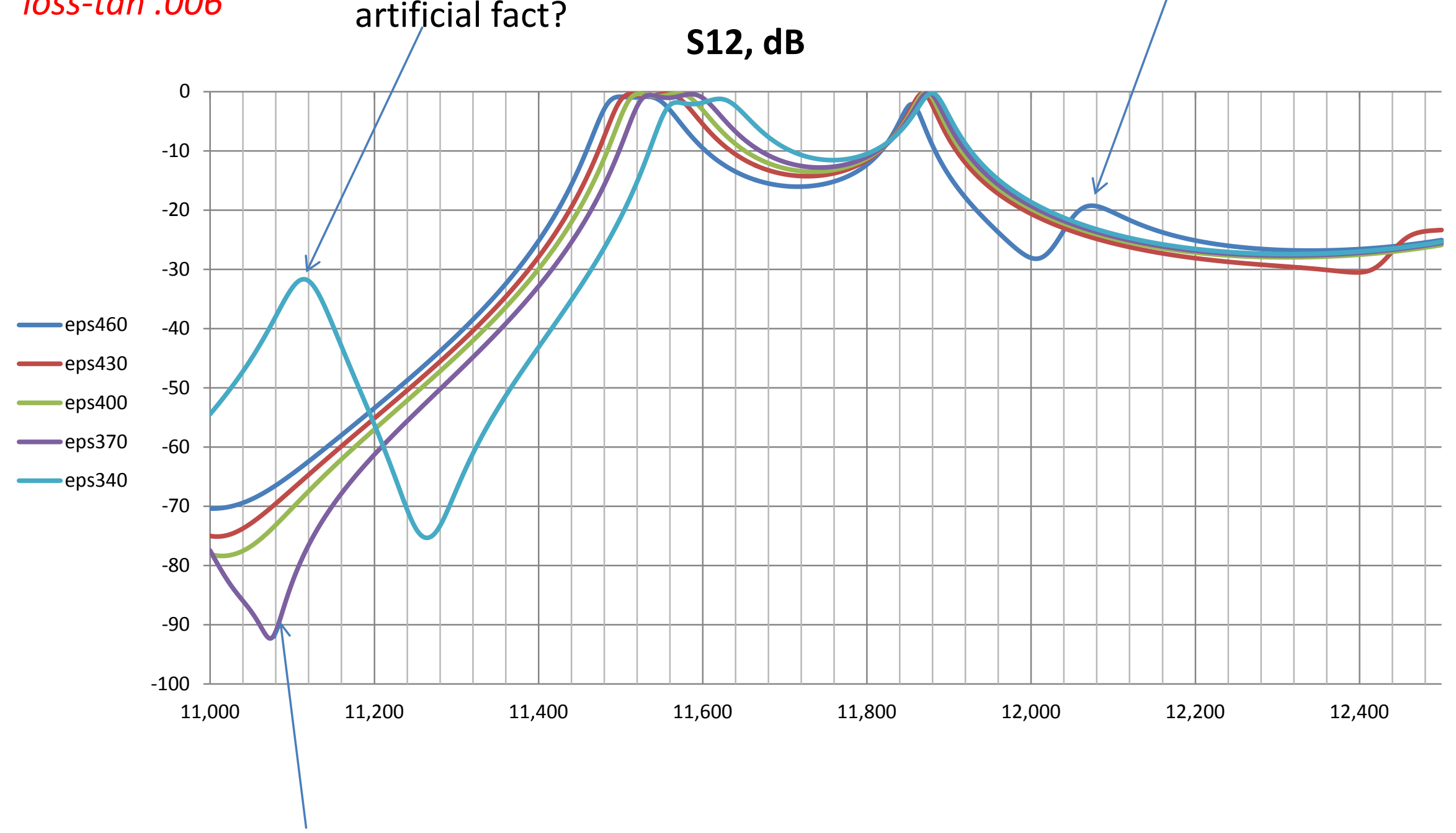

Is this an artificial fact? 


\section{FE rings,}

no grooves

\section{epsilon changes}

loss-tan .006

\section{S11,dB}

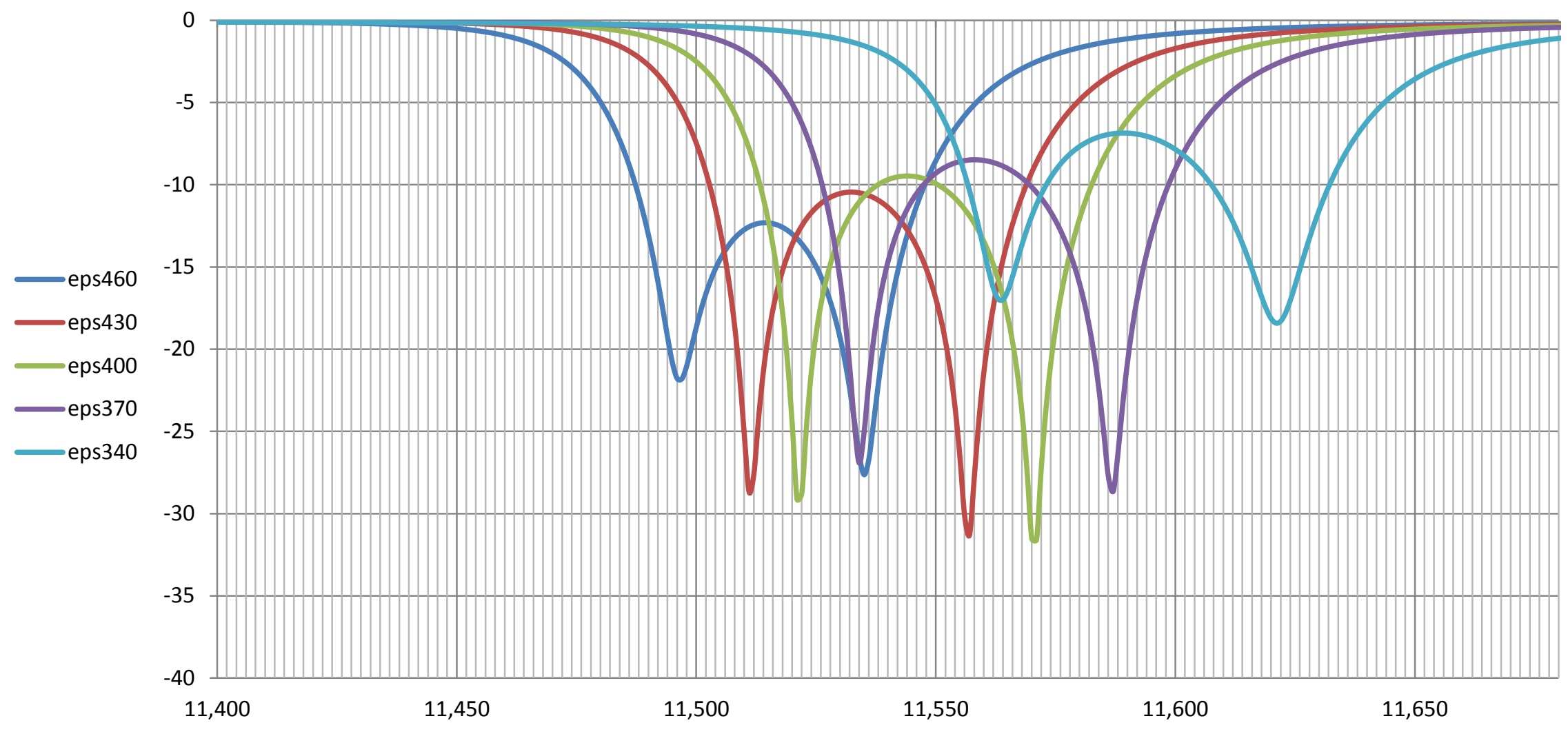




\section{FE rings,}

no grooves

\section{epsilon changes}

loss-tan .006

\section{S11,dB}

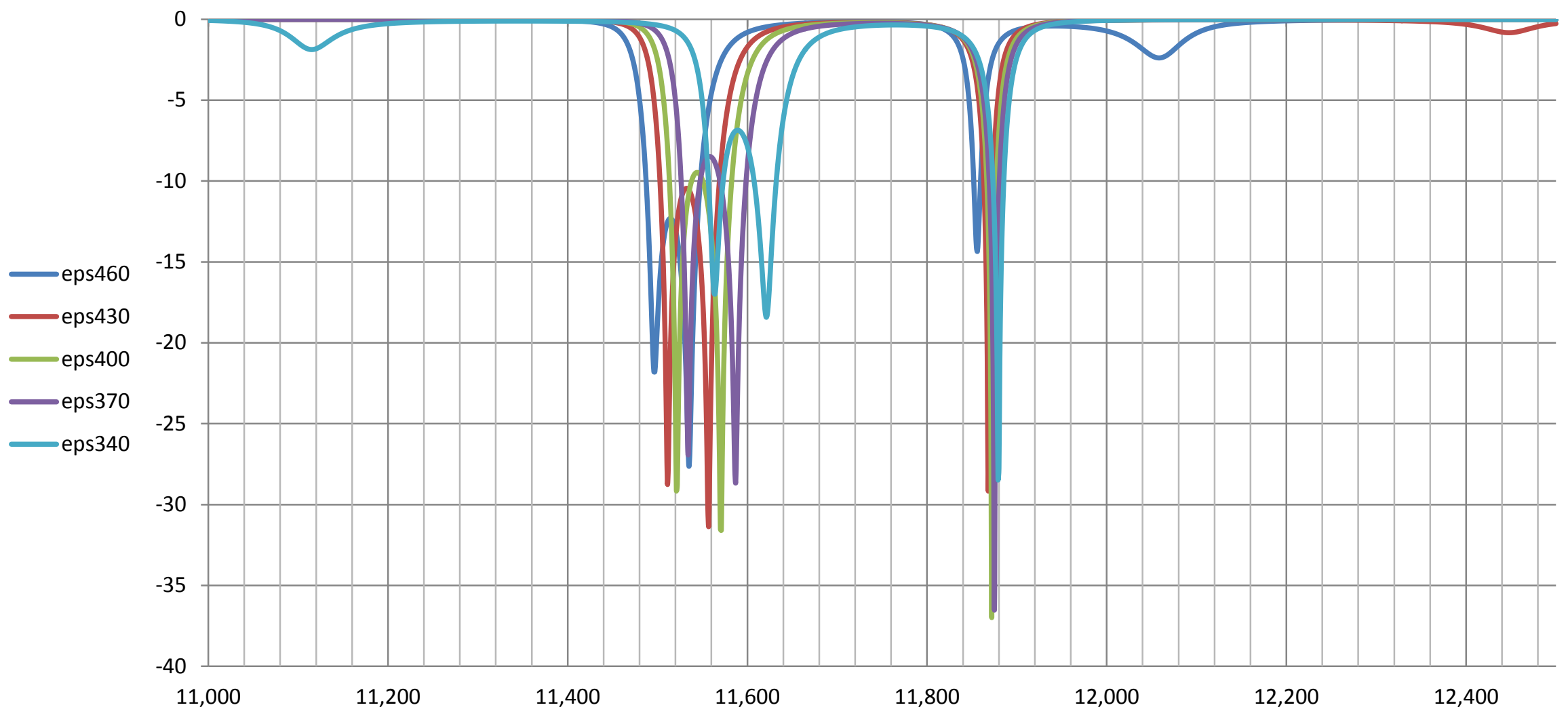


Things to think of:

1) Validity of simulation results: several simulations near eps $\sim 340$ should be done to make sure of the transmission behavior

2) In experiment: collect "pulse compression data" for different bias voltages, which - of course - corresponds to different epsilons

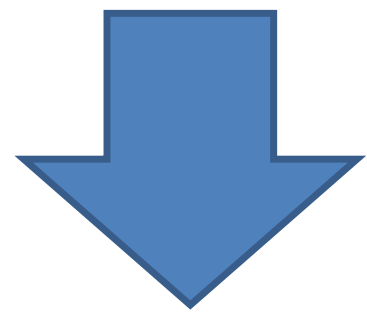




\section{Comparison $\mathrm{w} /$ all aluminum rings}

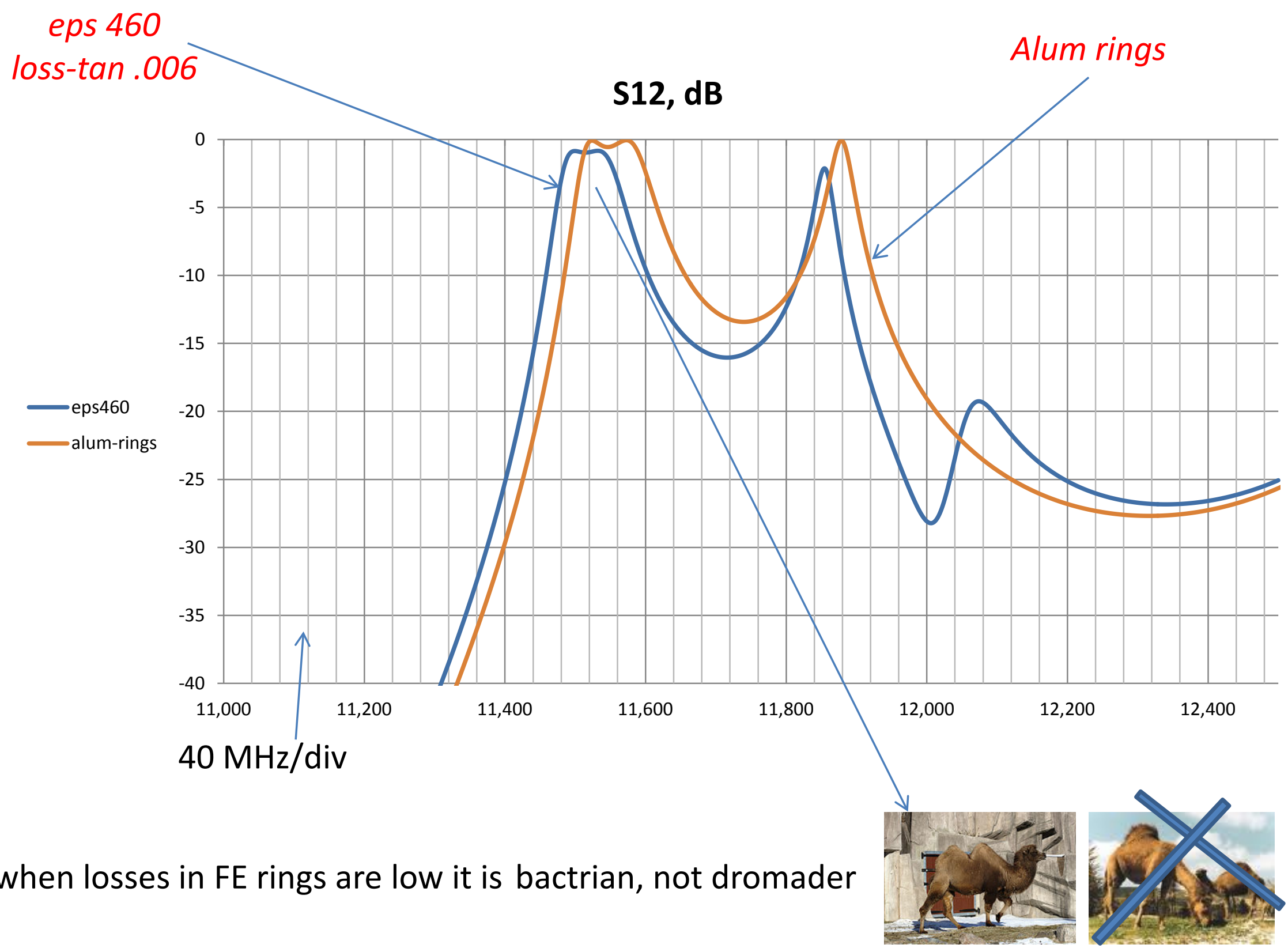




\section{Comparison $\mathrm{w} /$ all aluminum rings}

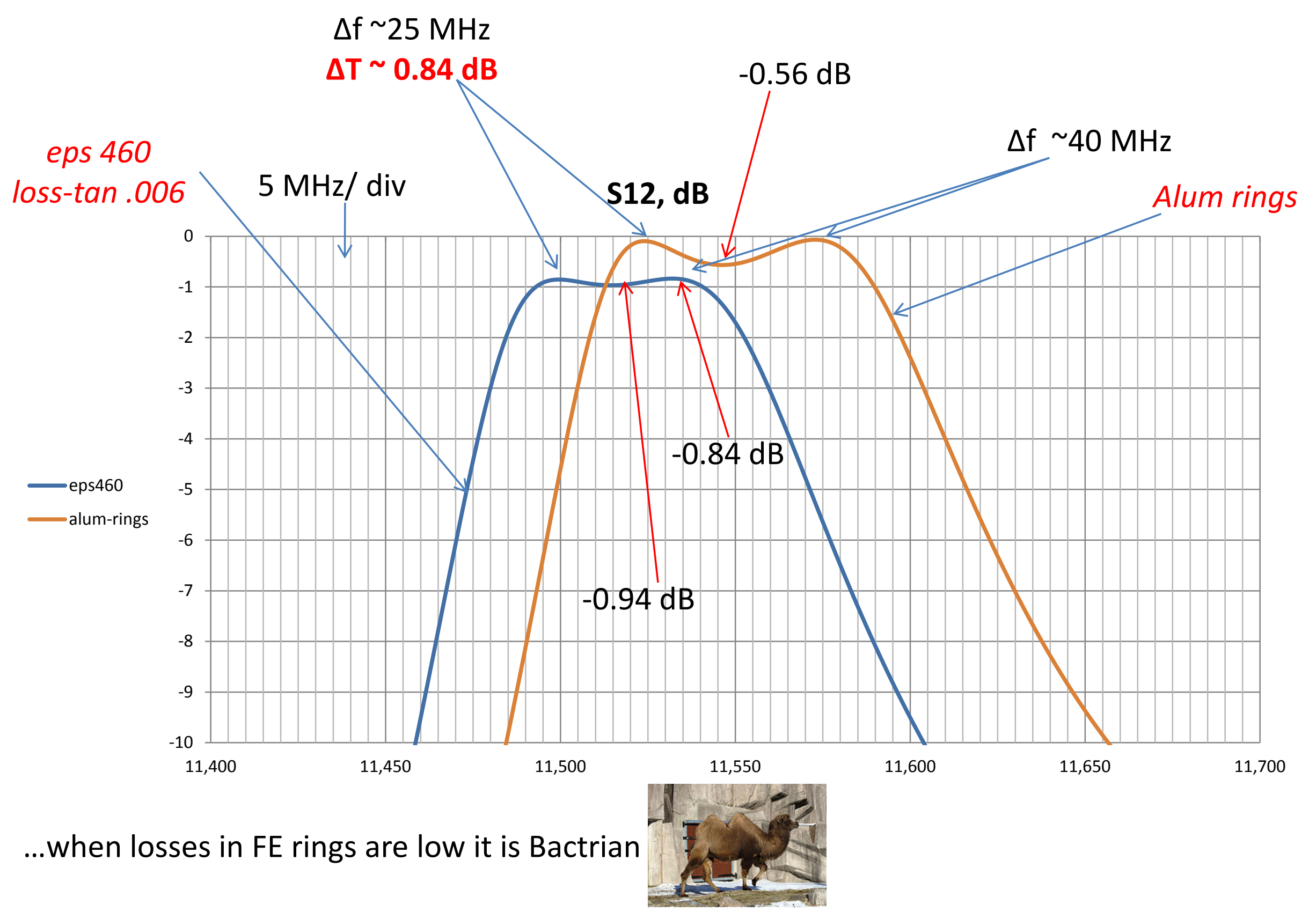




\section{Transmission w/ FE rings}

Q: the difference between transmission level is $(3.96-3.13)=0.84 \mathrm{~dB}$, and is as the expected difference [see the slide above] when the FE-losses are low
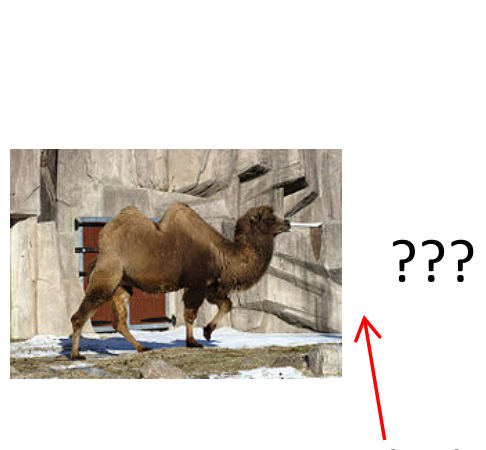

Transmission w/ alum rings

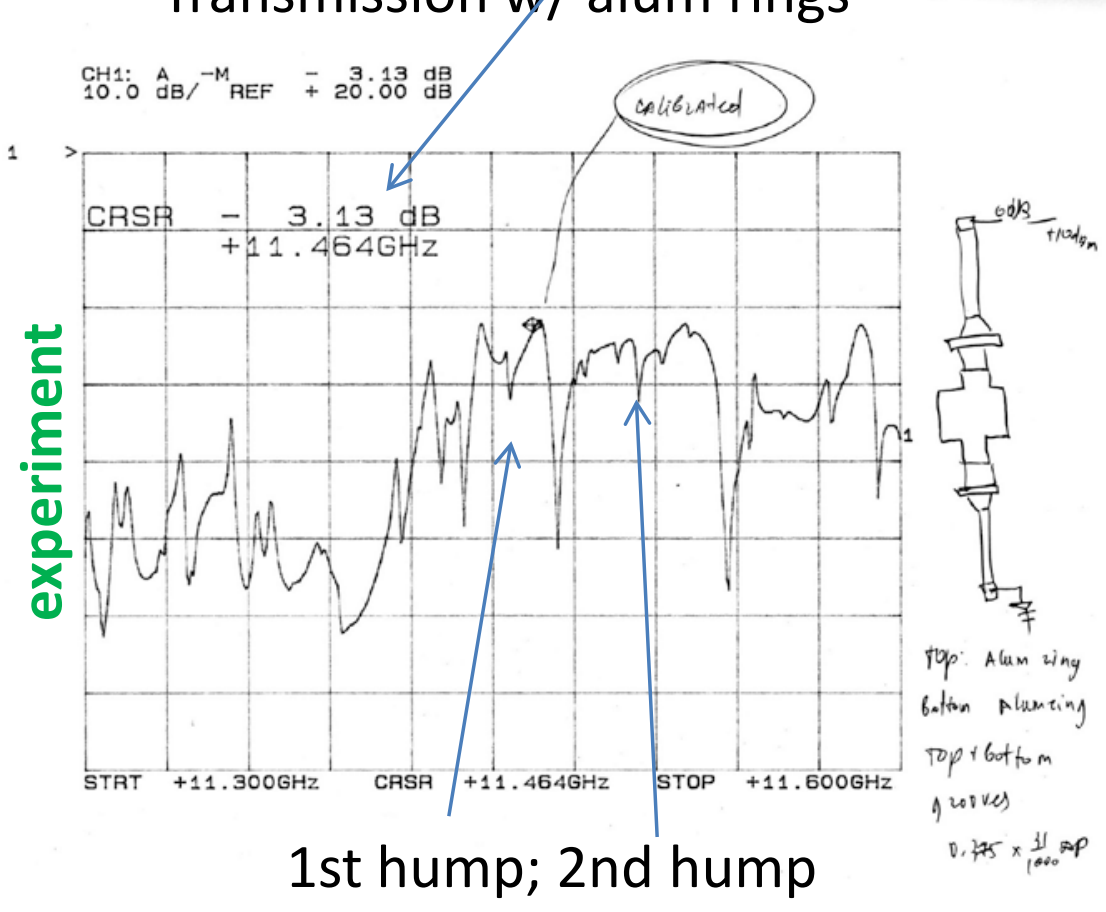

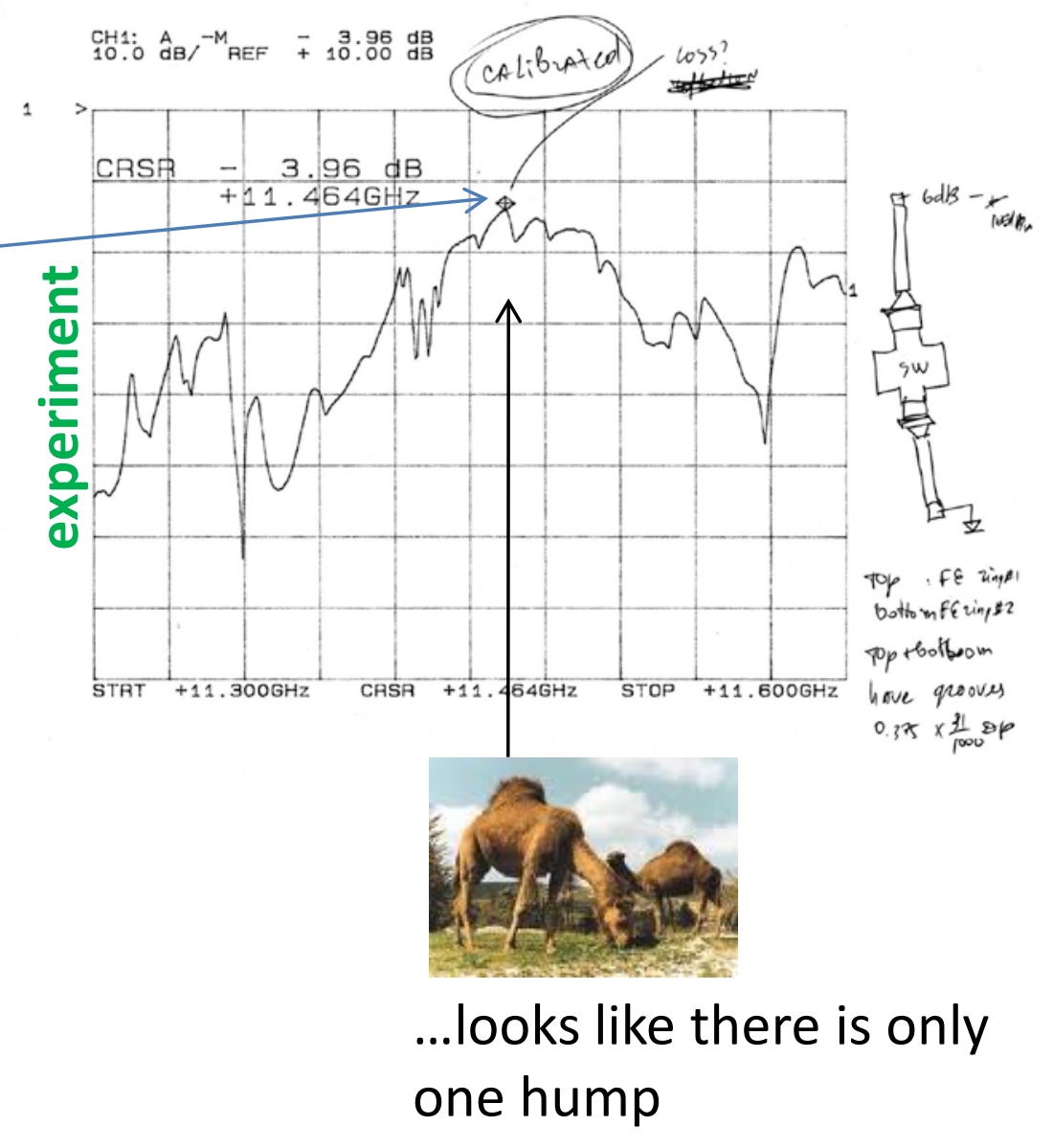

Q: however, a single hump is absent, which may suggest that the FE-losses are NOT low...

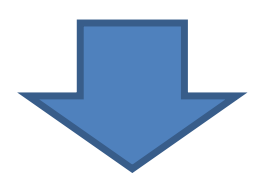




\section{Loss-tan \\ Chapter 3:}

changes Transmission

\section{of the Switch}

w/ FE Rings

(no grooves) 


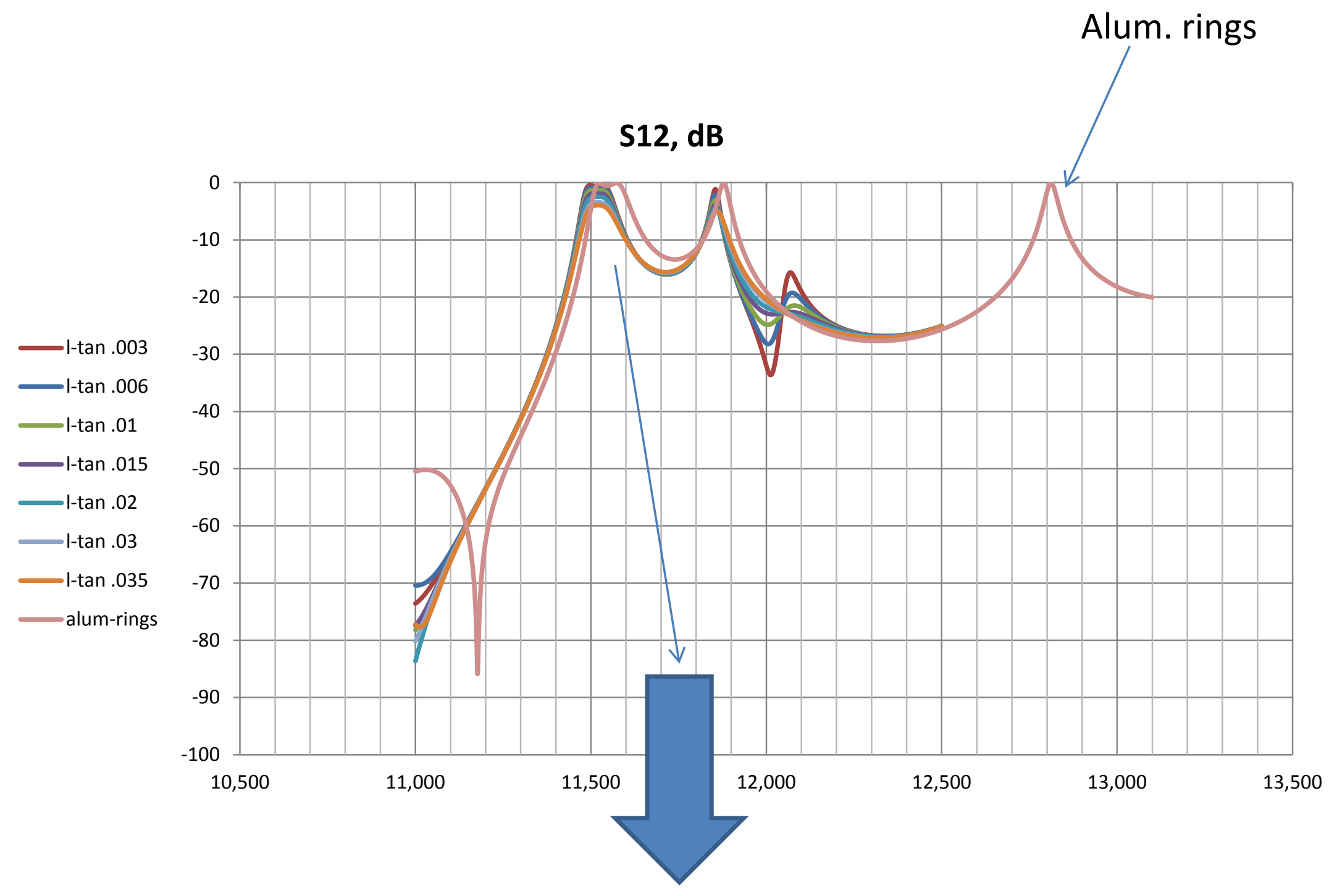




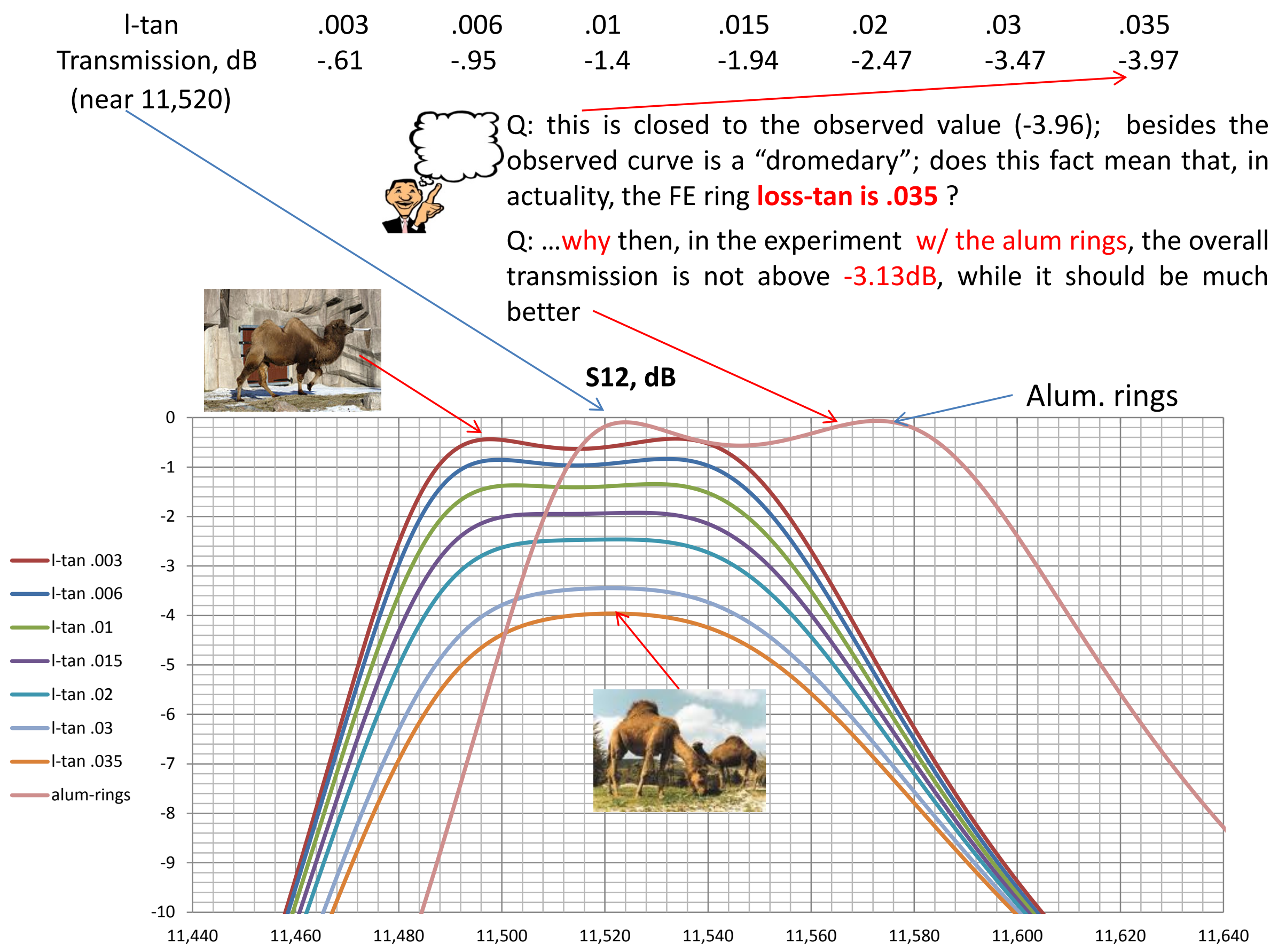




\section{Appendix I: \\ (Some notes)}

1) Calculation of the transmission for the Switch $w /$ alum rings are done $w /$ the copper side walls (58 MSimens/m) and the rings $\mathrm{w} / 29 \mathrm{MSimens} / \mathrm{m}$

2) Calculation of the transmission for the Switch w/ FE rings are performed w/ the ideally conductive walls, but a comparison [see below] $\mathrm{w} / \mathrm{a}$ version whose walls are from copper (at $58 \mathrm{MSimens} / \mathrm{m}$ ) show not difference (just a very small one)

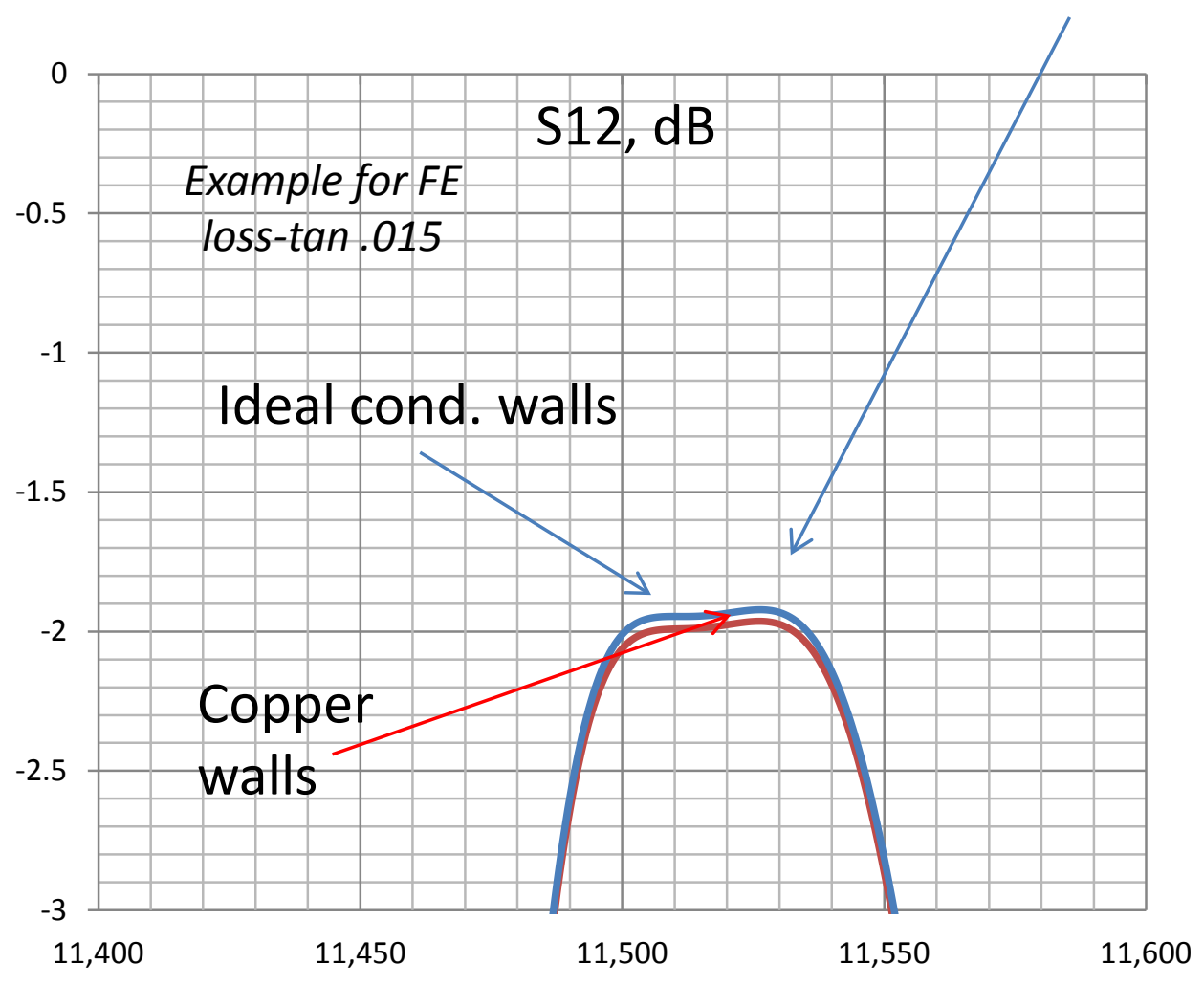




\section{X-band Switch}

with two aluminum rings 


\section{Scheme to measure transmission}

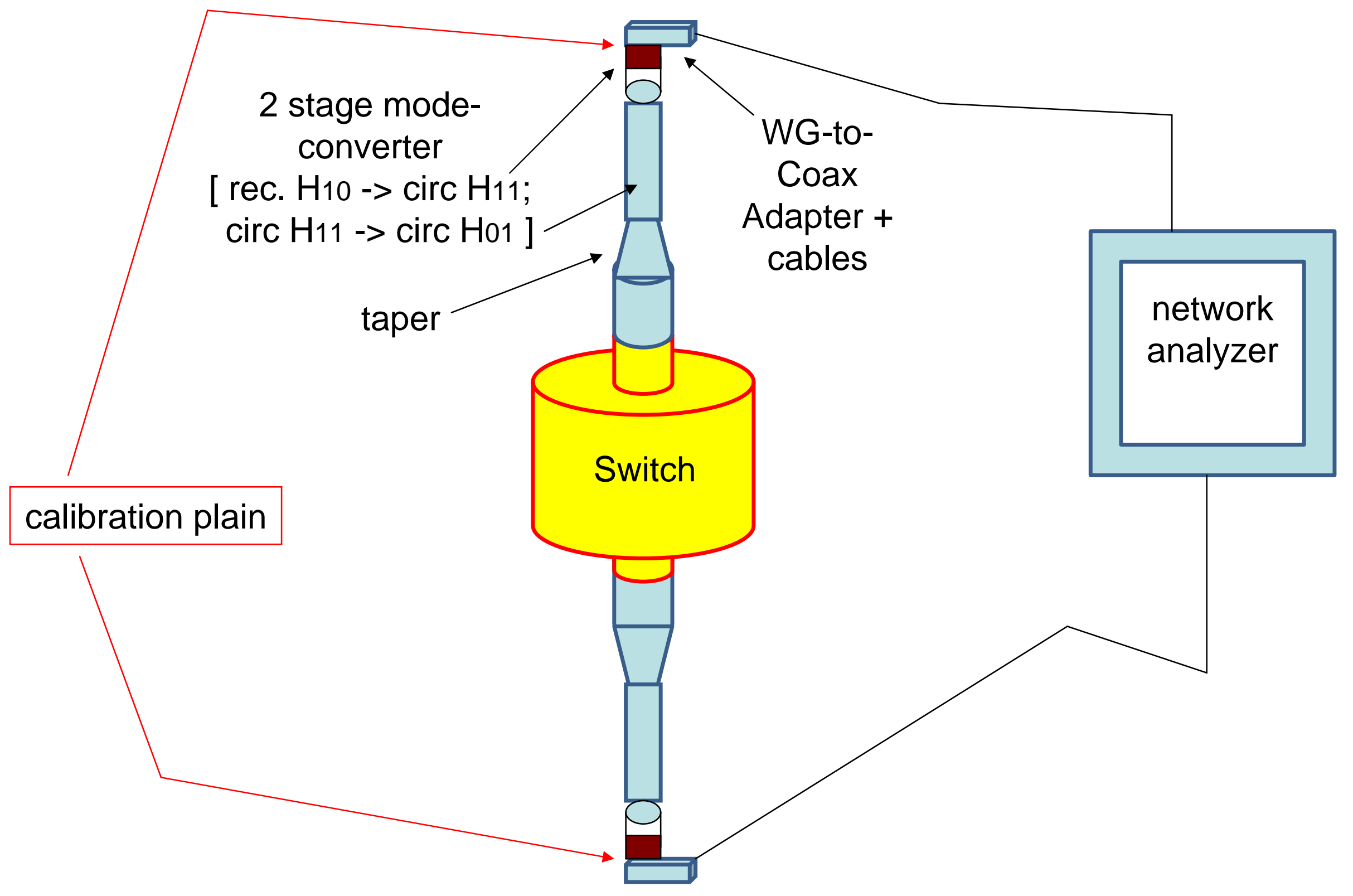




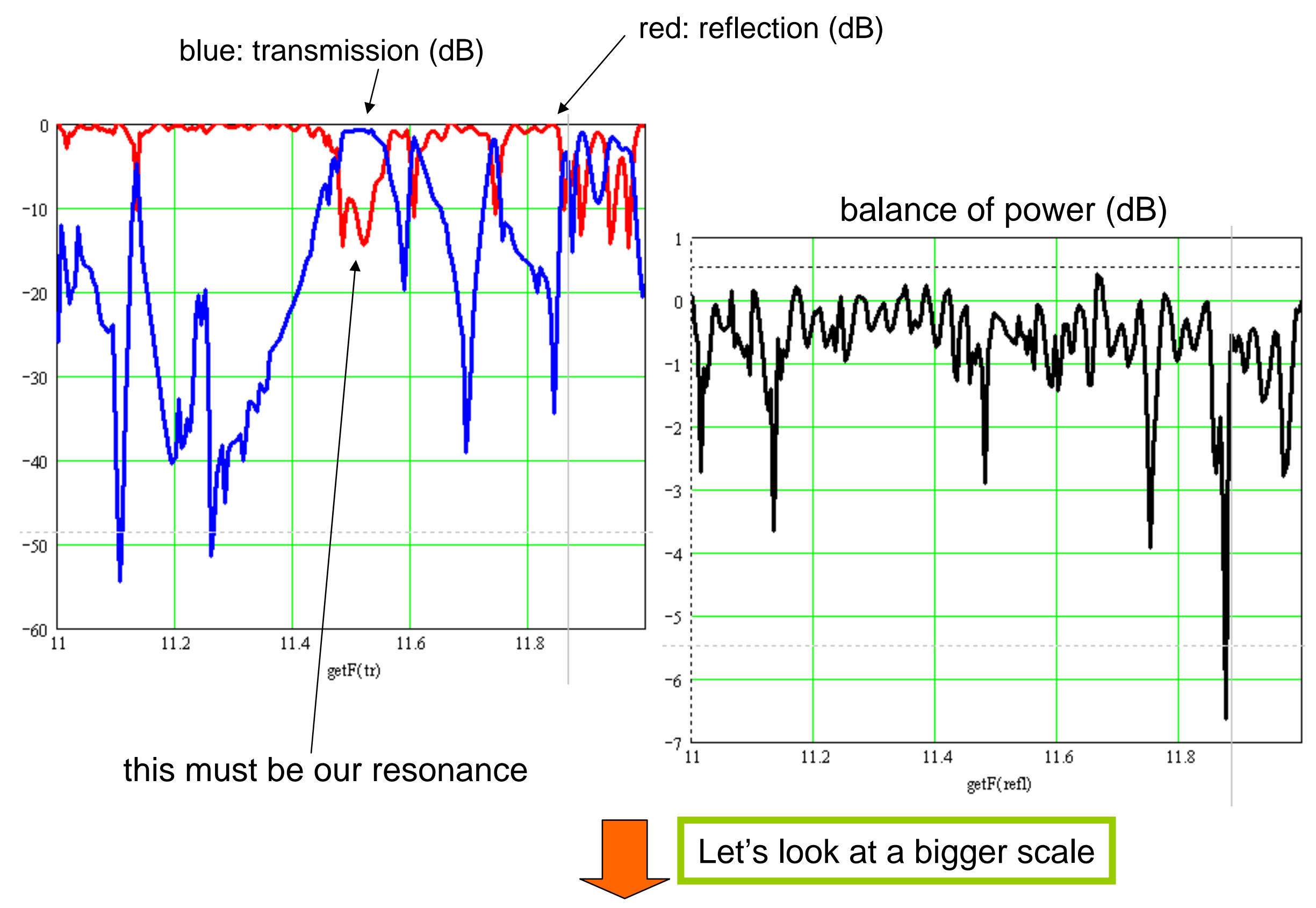




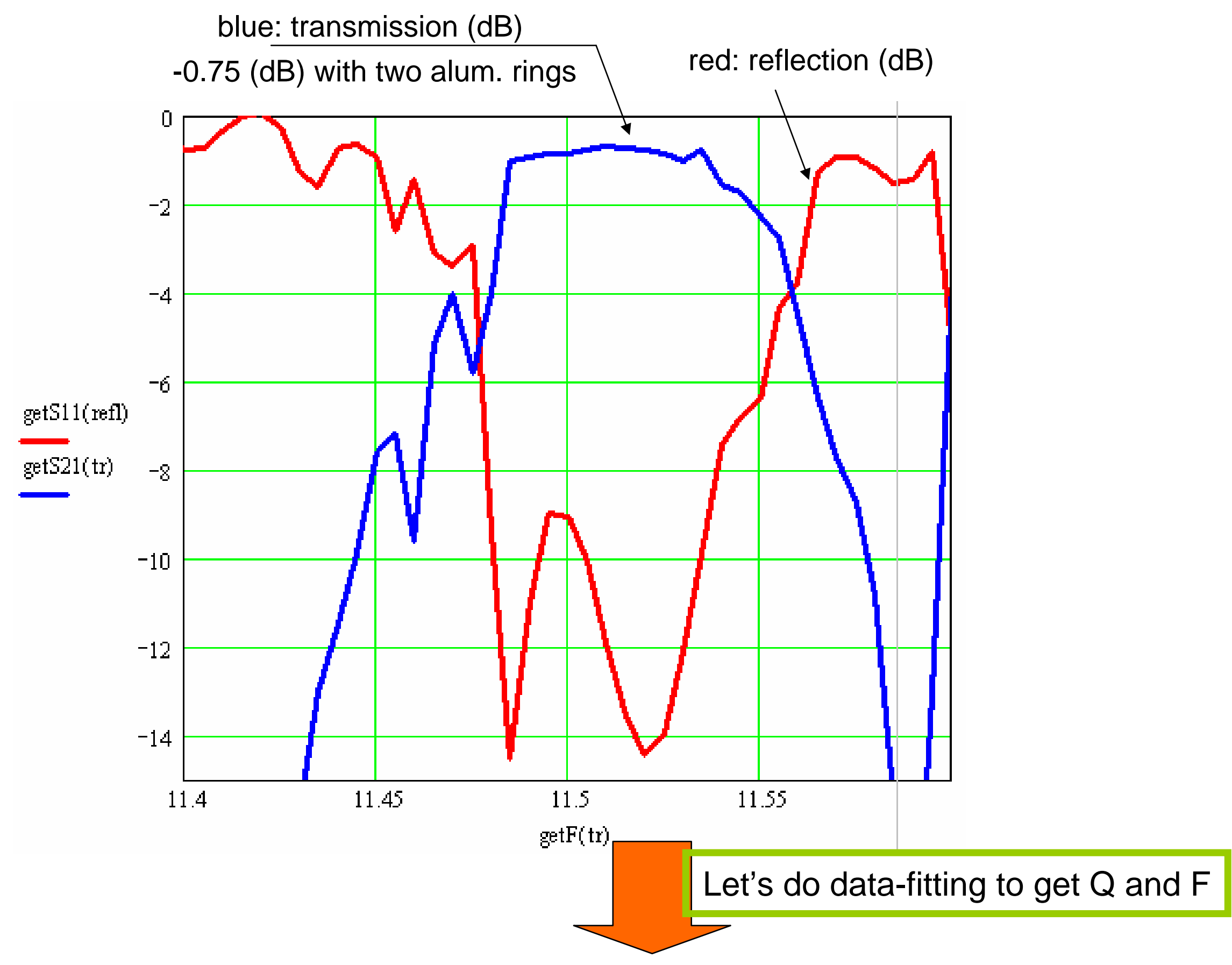


red: measured reflection $(\mathrm{dB})$

getS11(refl)

getReflCurve(getF(refl) ,- $14.431,11.52,105$ )

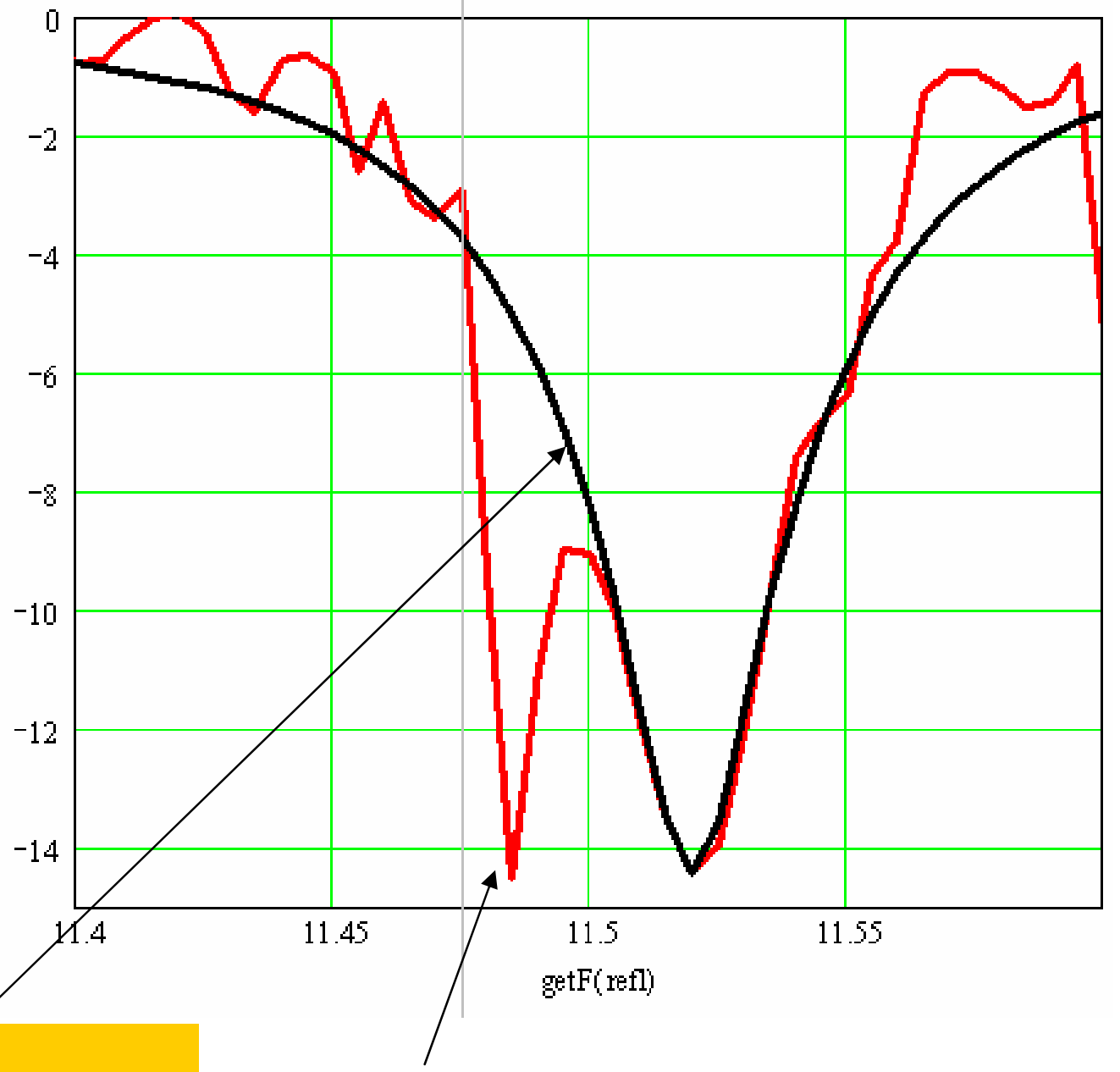

Black: data-fit

$F_{0}=11.520 \mathrm{GHz}$;

$\mathrm{Q}_{\text {total }}=105$

Bandwidth = $110 \mathrm{MHz}$

some resonance because of modeconverters 
Observed bandwidth $110 \mathrm{MHz}$ matches [sort of] $\mathrm{w} /$ the predicted $(\sim 135 \mathrm{MHz})$

...but the frequency $(11,520 \mathrm{MHz})$ is way off from the needed $(\mathrm{dF}=+100 \mathrm{MHz})$

... this cannot be tuned by grooves [by the way] because grooves may tune only by $-50 \mathrm{MHz}$, and there are already some made;

... we need to recess the back wall a bit (to change the cavity's total length), leaving the gap where the rings go intact;

... before that we need to do measurements $\mathrm{w} /$ the FE rings (of course) to be sure.

\section{Simulations by CLANS}

$\mathrm{w} /$ the aluminum ring

$\mathrm{F}=11,372 \mathrm{MHz}$

$T=-0 \mathrm{~dB}$

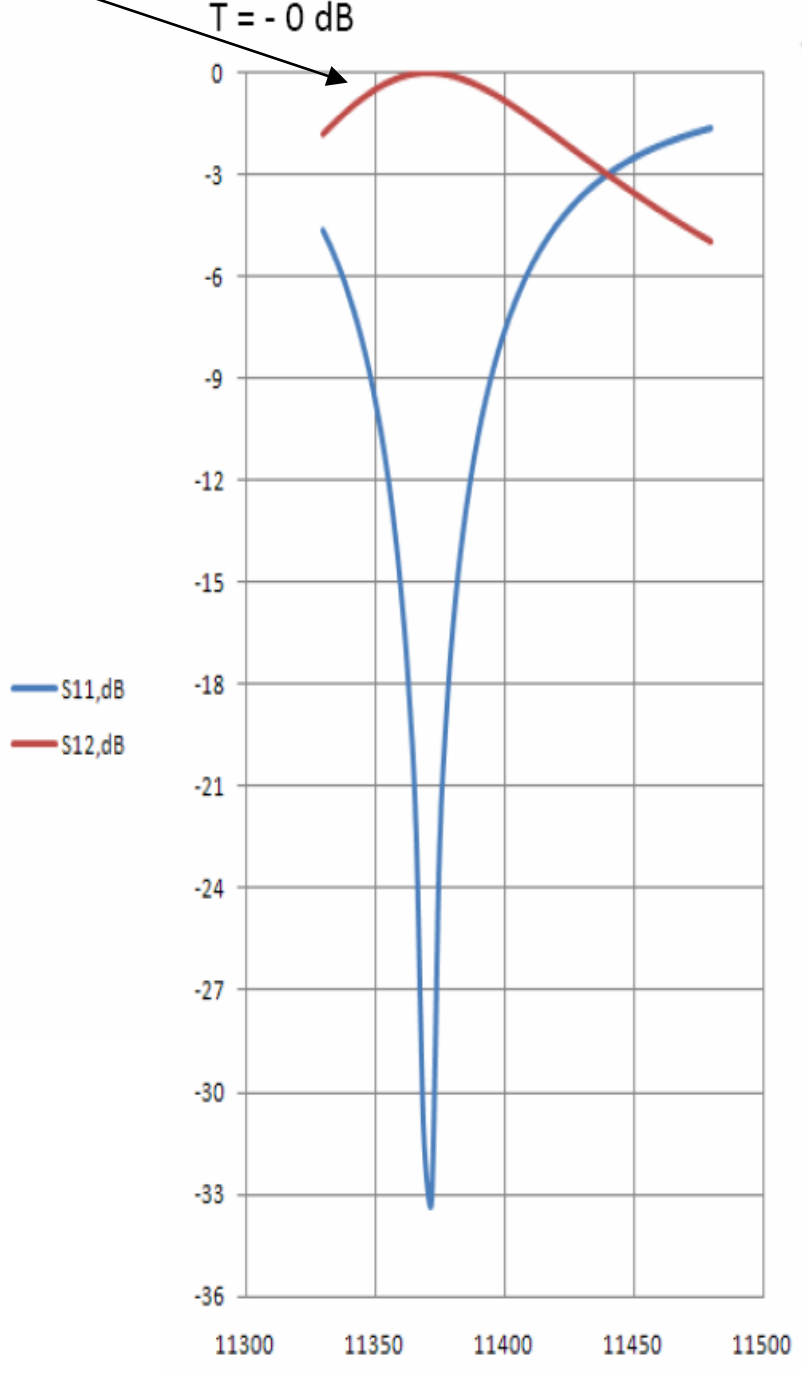


Appendix: some pictures

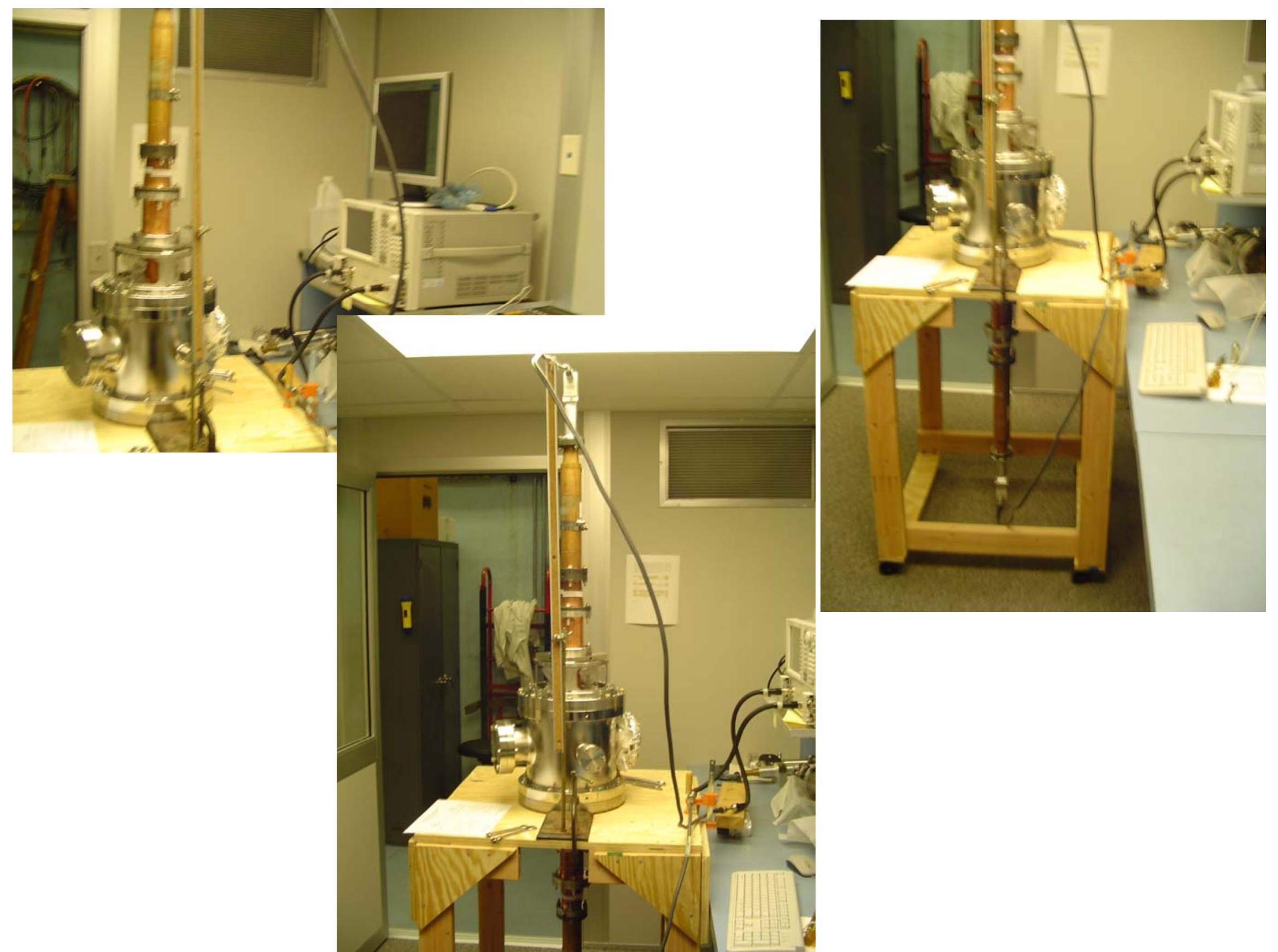




\subsection{GHz Switch with $\mathrm{FE}$ rings}

1. The numbers of points per sweep were increased from 201 to 3601 giving a good resolution for all the features.

2. The new transmission curves are shifted by $\sim 20 \mathrm{MHz}$ down relative to the old ones; this was expected.

3. The flat top on the transmission curves remains; it is about $\sim 40 \mathrm{MHz}$ wide.

4. The reflections... S11 has its left dip larger, and S22 has its right dip larger; two curves look sort-of-symmetrical with respect to $11.5 \mathrm{GHz}$.

5. The reflection is as low as $-15 \mathrm{~dB}$ (or better).

6. From the balance of powers, it seems that the presence of FE rings (one per cavity) has increased losses by $0.56 \mathrm{~dB}$ 
Red: transmission (S21) with alum. rings

Blue/Black: transmission (S12/ S21) with FE rings

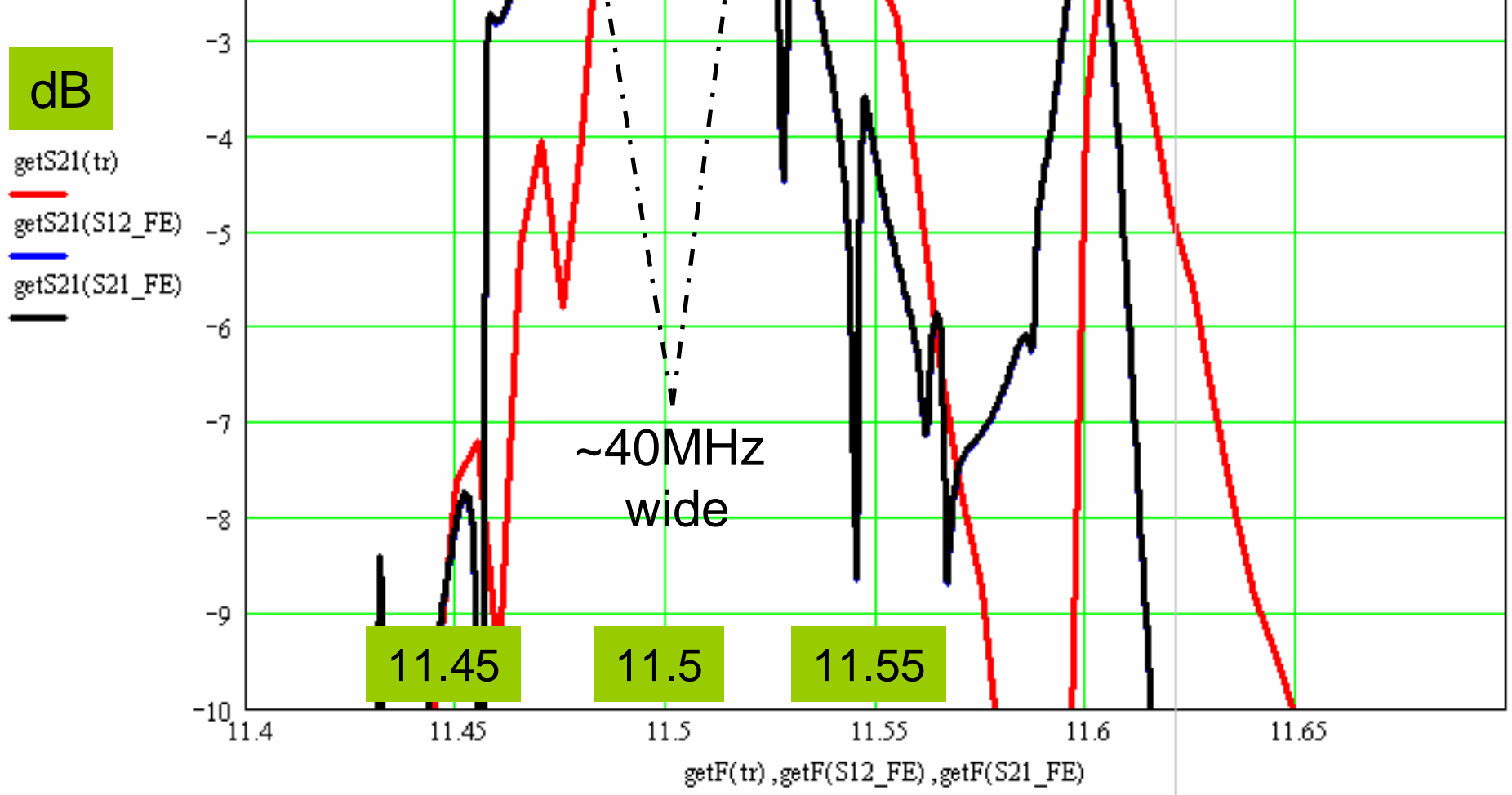

$\mathrm{GHz}$ 
Red: reflection (S11) with alum. rings

Blue/Black: reflection (S22/ S11) with FE rings

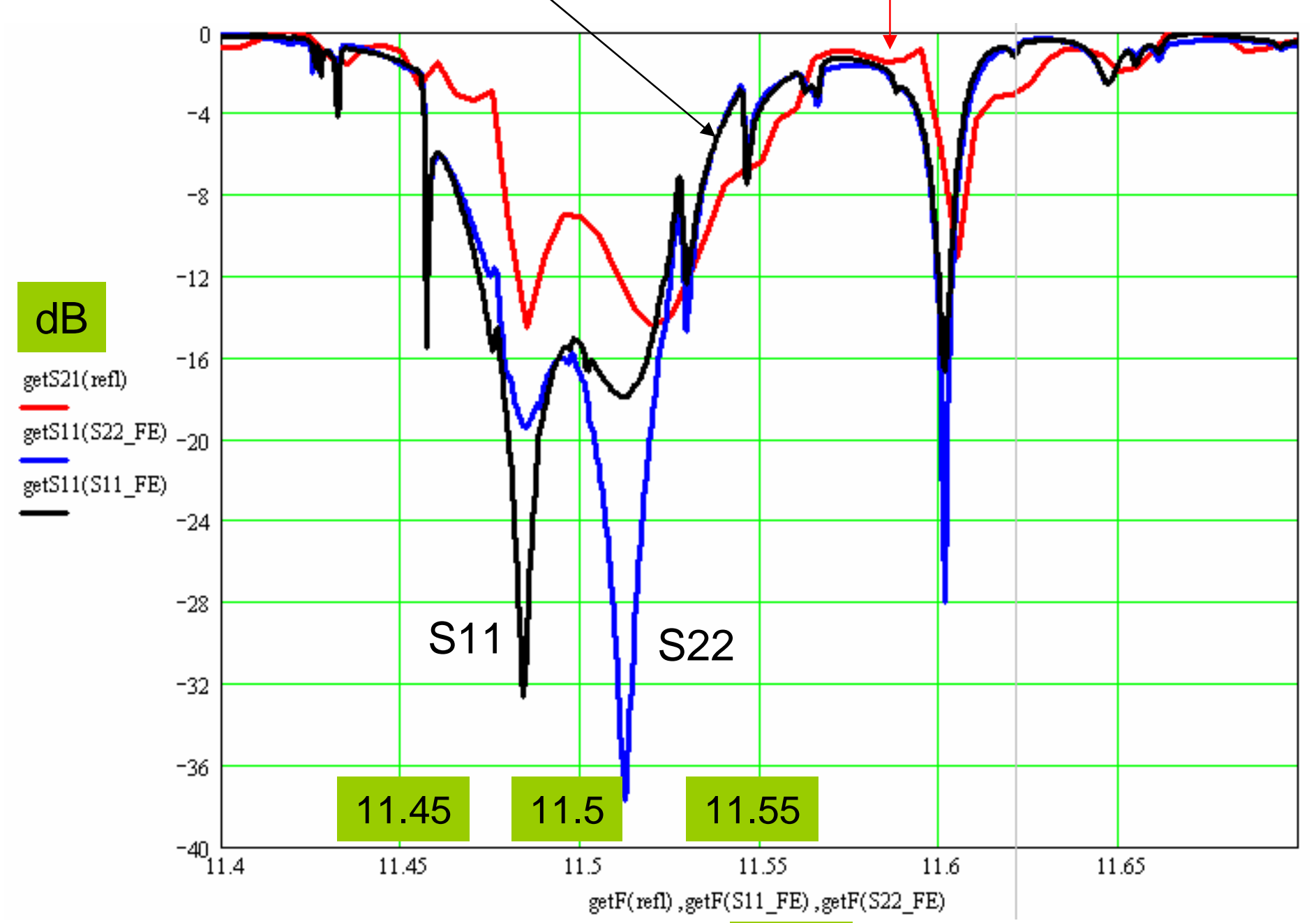

$\mathrm{GHz}$ 
Red: balance with alum. rings

Blue/Black: balance (port1/ port2) with FE rings

\section{$\mathrm{dB}$}

getBalance(refl,tr)

getBalance(S22_FE, S12_FE) getBalance(S11_FE,S21_FE)

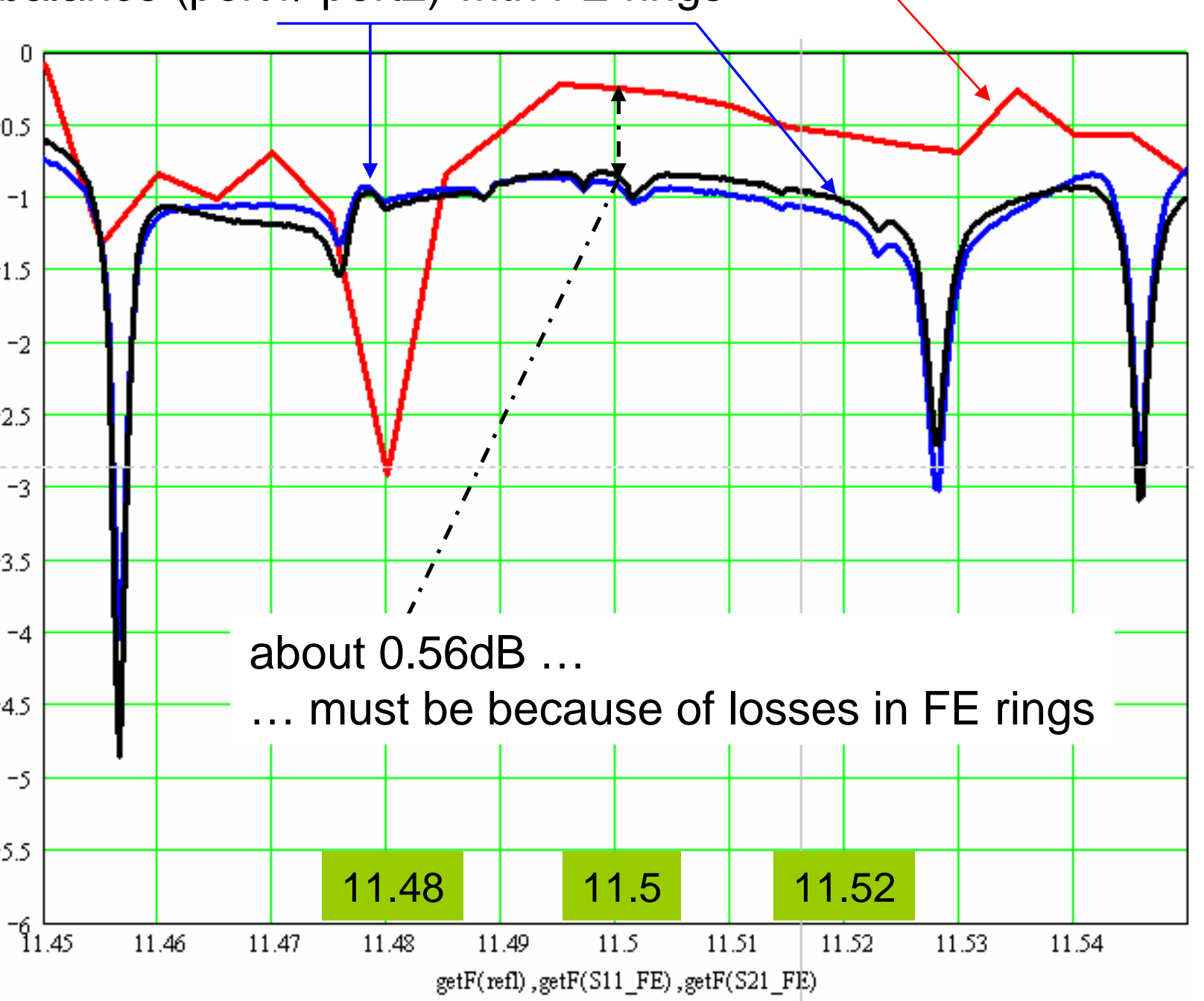

$\mathrm{GHz}$ 
The frequency shift $(-32 \mathrm{MHz})$ is as predicted by simulations for the groove OD92mm, ID57mm, 0.8mm Deep

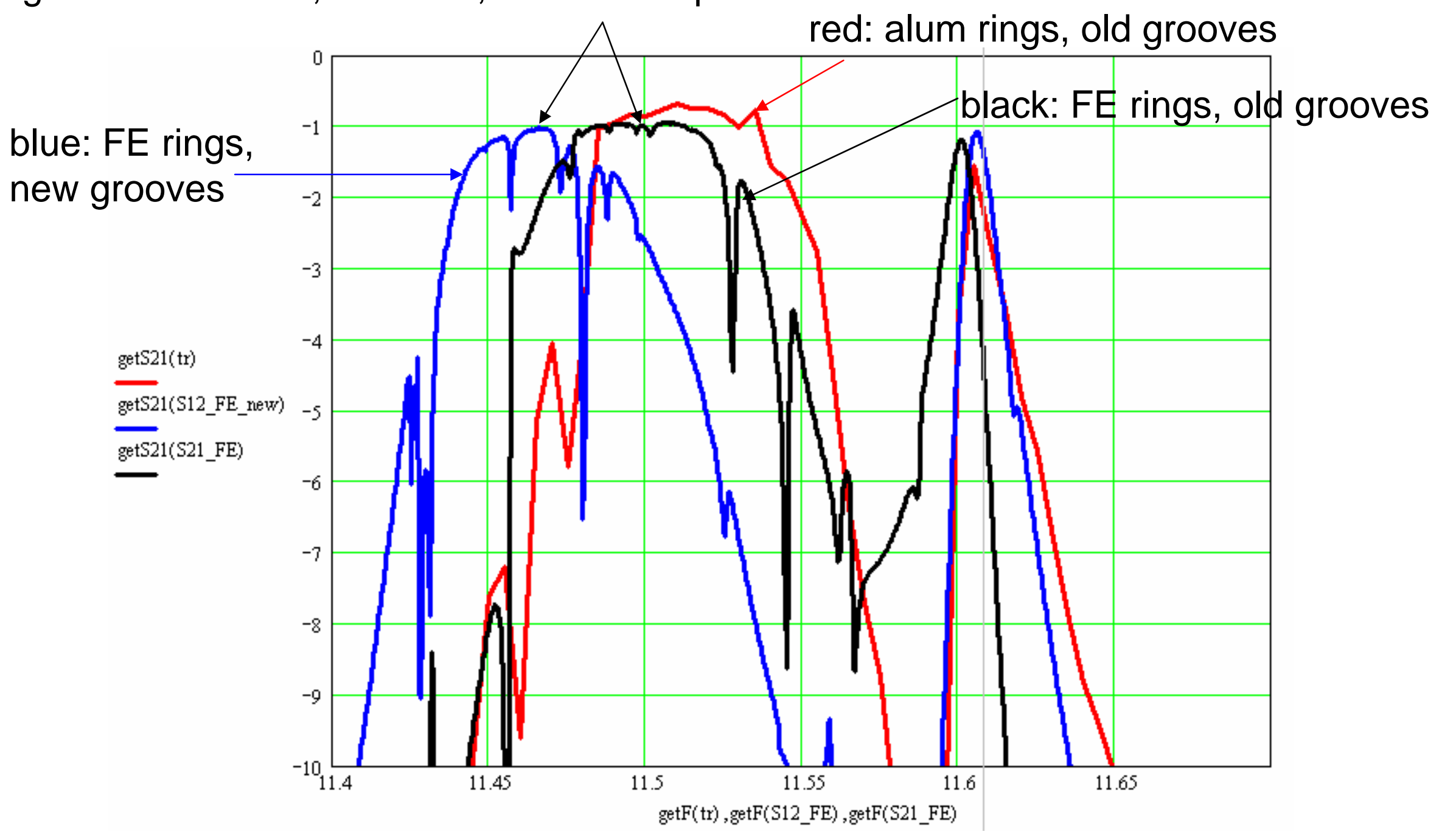


Moved by $70 \mathrm{MHz}$, while the prediction was $71 \mathrm{MHz}$

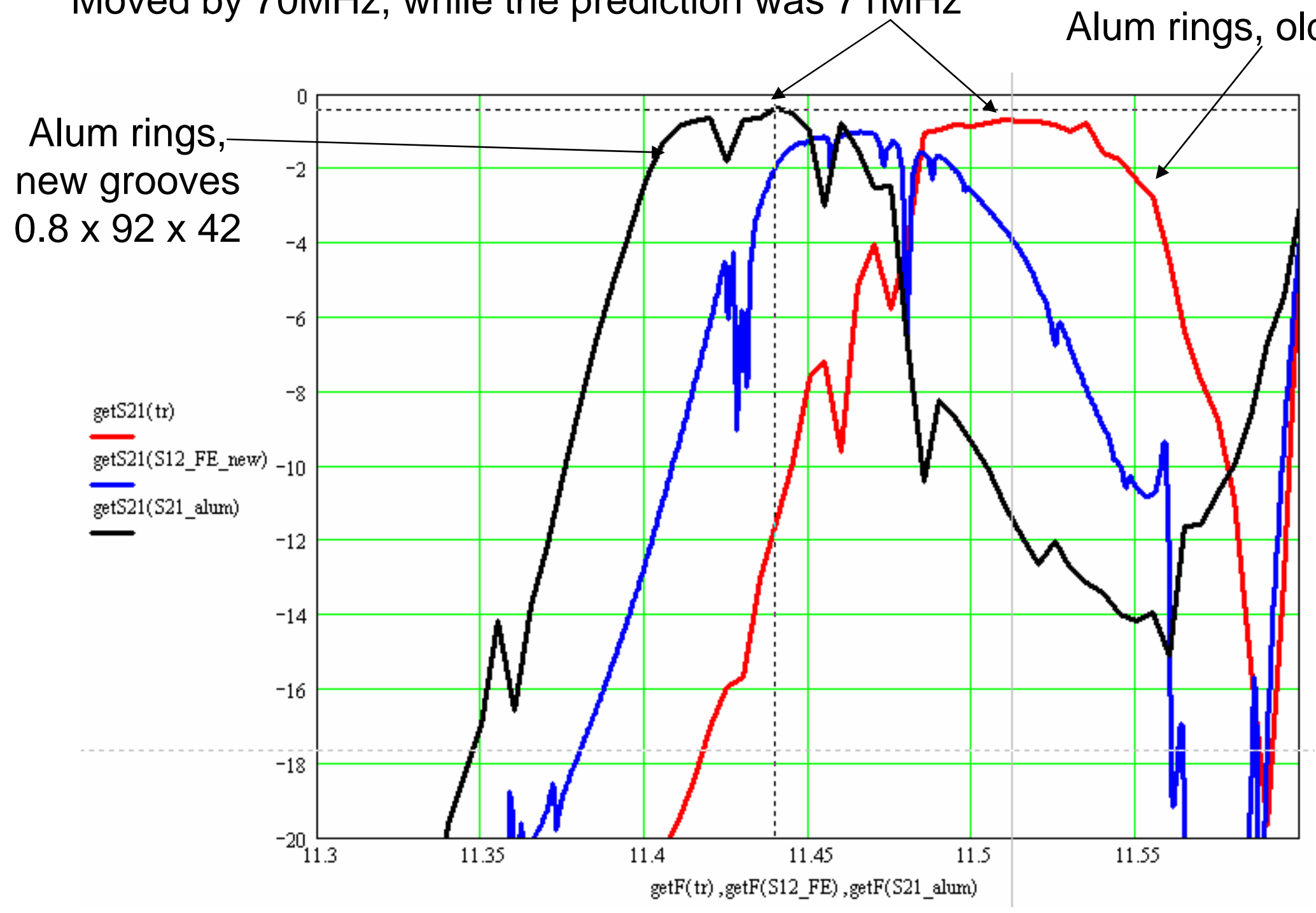


new grooves 1.3DPT x 98OD x 42ID mm

\section{alum rings}

measured $11367 \mathrm{MHz}$ (vs predicted 11369)

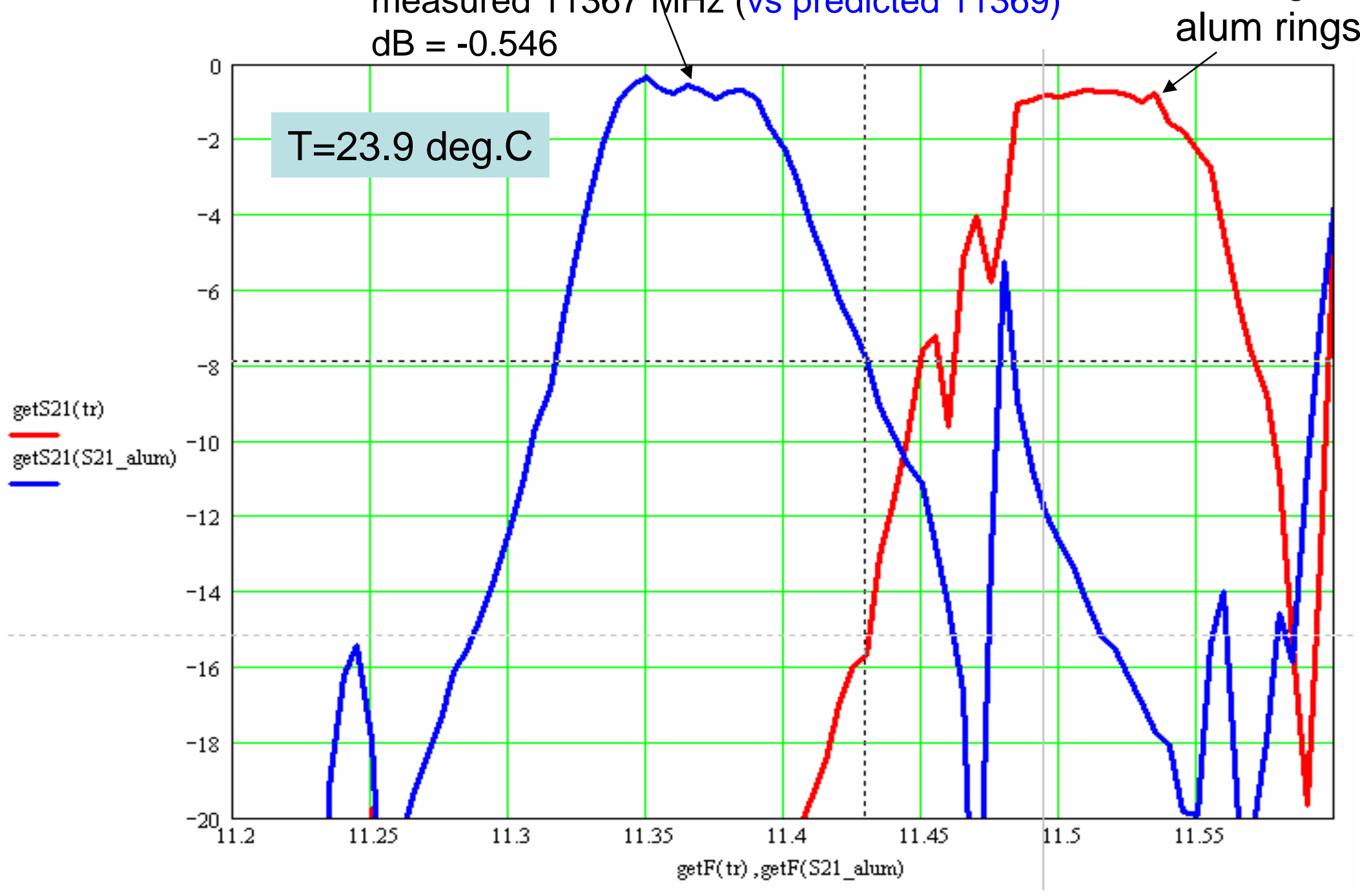

old original grooves, alum rings

getS21(tr)

getS21(S21_alum)

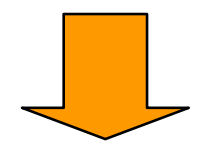




\section{"interpolated" for FE rings}

$95 \mathrm{kV}$

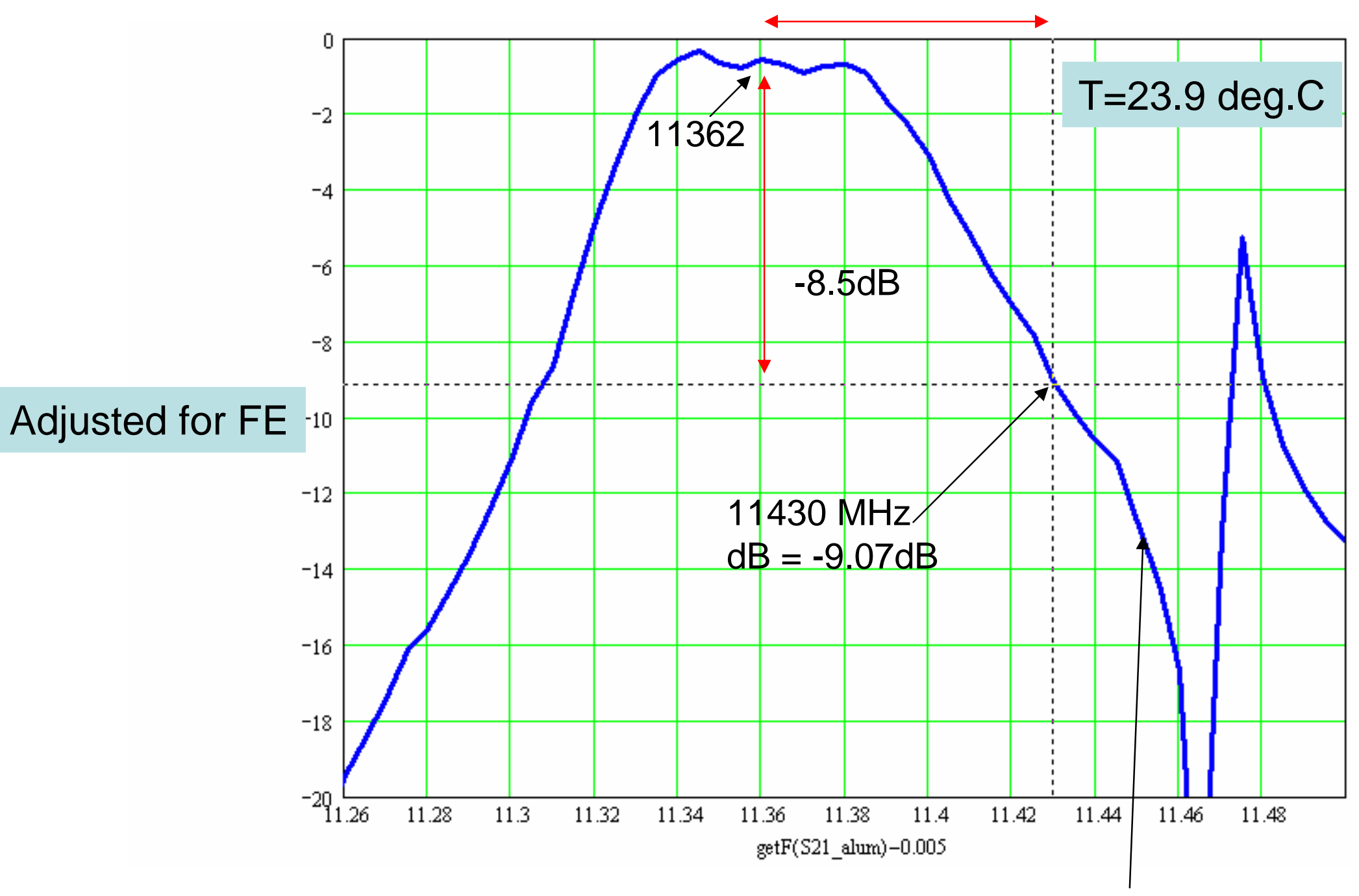

move this point to 11430 , and operate at $T=35 \mathrm{deg}$. $\mathrm{C}$

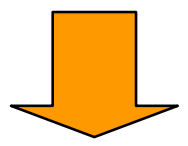


11340 at $24 \operatorname{deg} C$ proposed for FE rings

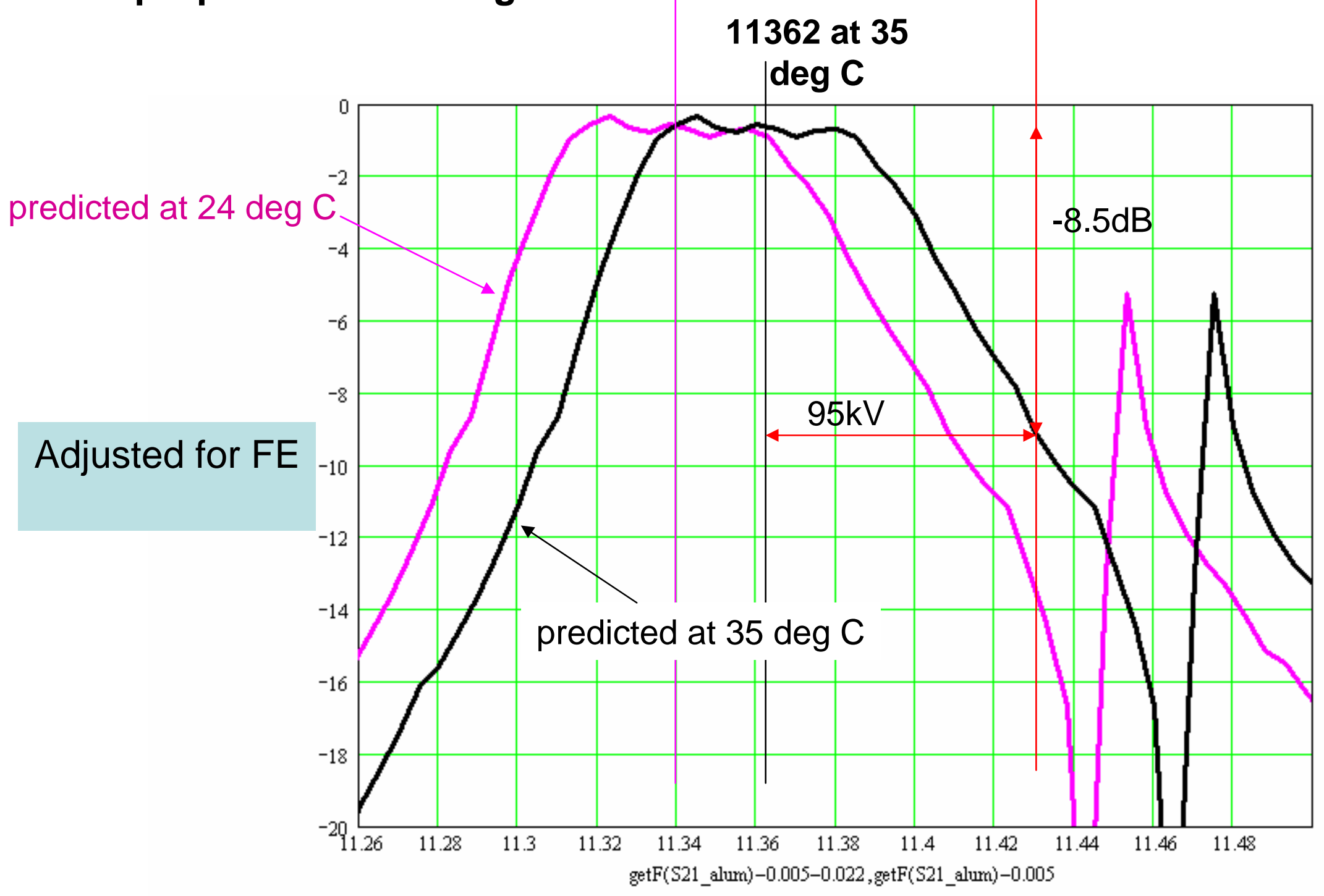


new grooves 1.3DPT x 98OD x 42ID mm

alum rings

measured $11367 \mathrm{MHz}$ (vs predicted 11369)

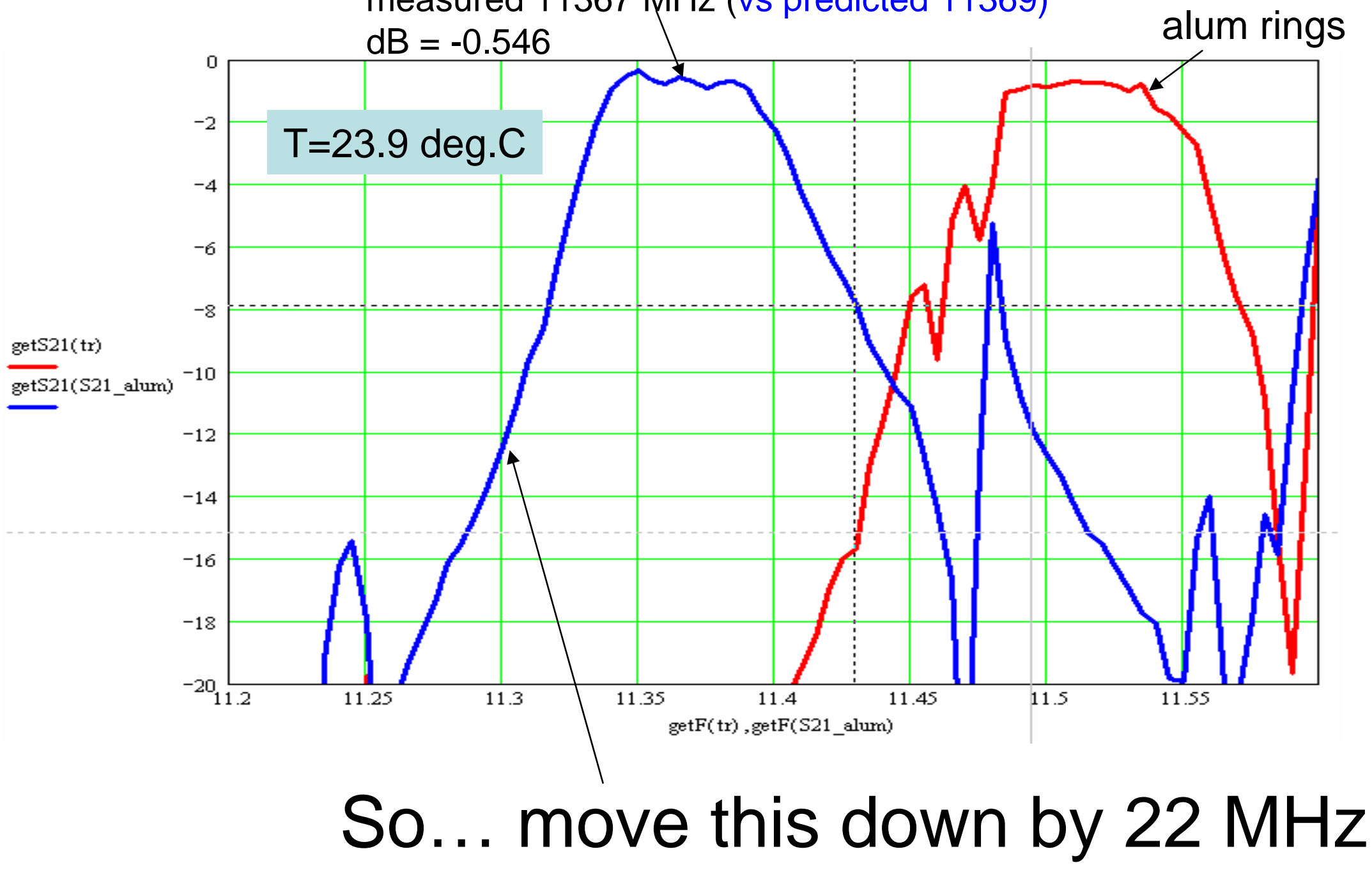

$(11367 \rightarrow 11345) \ldots$ simulation are needed 


\section{T_set $=35$ deg; T_bucket $=34.3 \mathrm{C} ; \mathrm{T} \_$structure $=30.3 \mathrm{C}$}

FE rings are used

S12_fe $:=$ READPRN $(" 12$ apr10_tr_s12_fe_tb_34C3.csv")

$\mathrm{S} 21$ fe $:=$ READPRN("12apr10_tr_s21_fe_tb_34C3.csv")

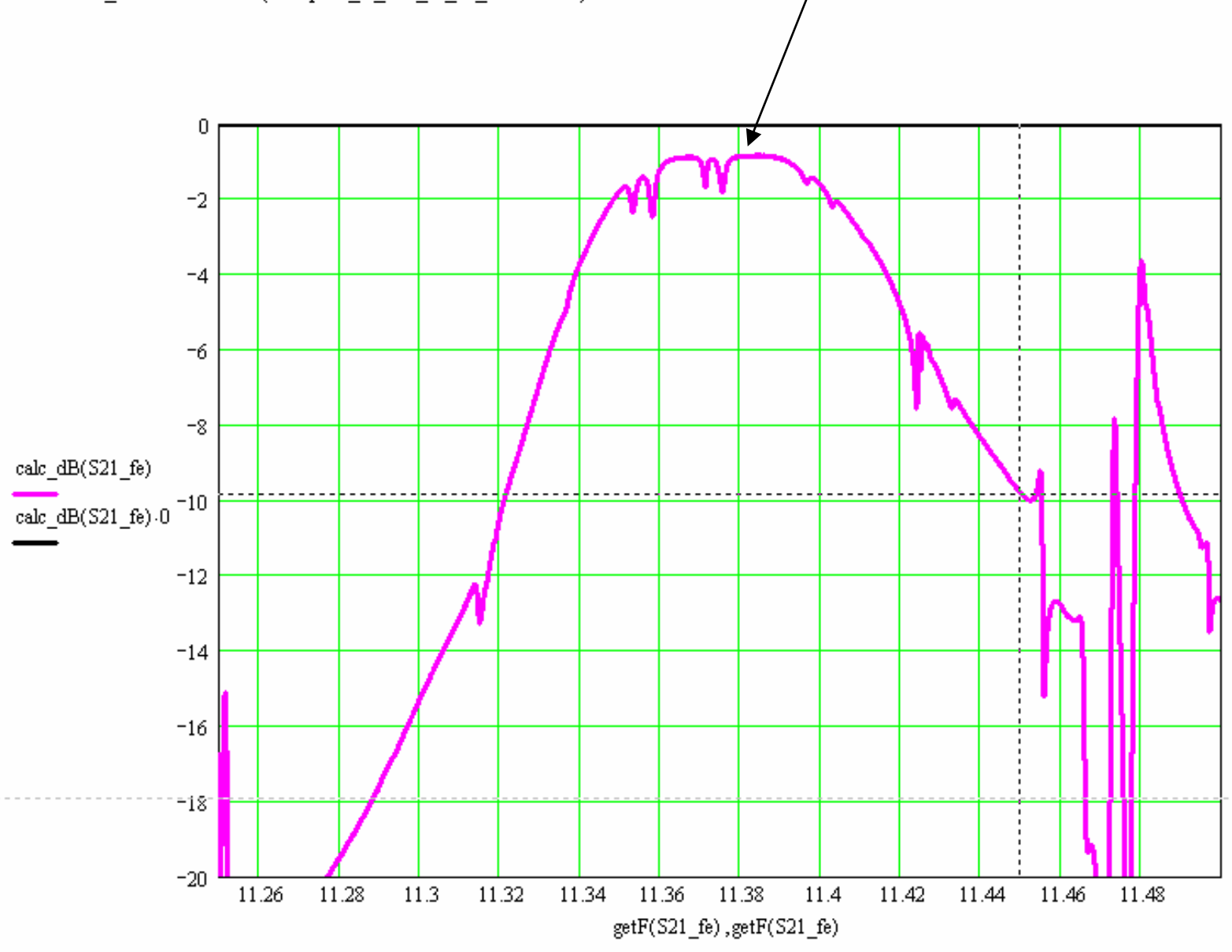

$68+382=450$

$450-430=20$

$376-362=14$

$30.3-23.9=6.4$

$\frac{14}{6.4}=2.1875$

$0.86+8.5=9.36$

\begin{tabular}{|c|c|}
\hline X-Y Trace & $x$ \\
\hline$X$ Value 11.45 & Copy $X$ \\
\hline YVValue -9.8063 & Copy Y \\
\hline 『 Track Data Points & Close \\
\hline
\end{tabular}


T_set $=35$ deg; T_bucket $=34.3 \mathrm{C} ;$ T_structure $=30.3 \mathrm{C}$

FE rings are used; $F_{\text {middle }}=11.380 \mathrm{MHz}$

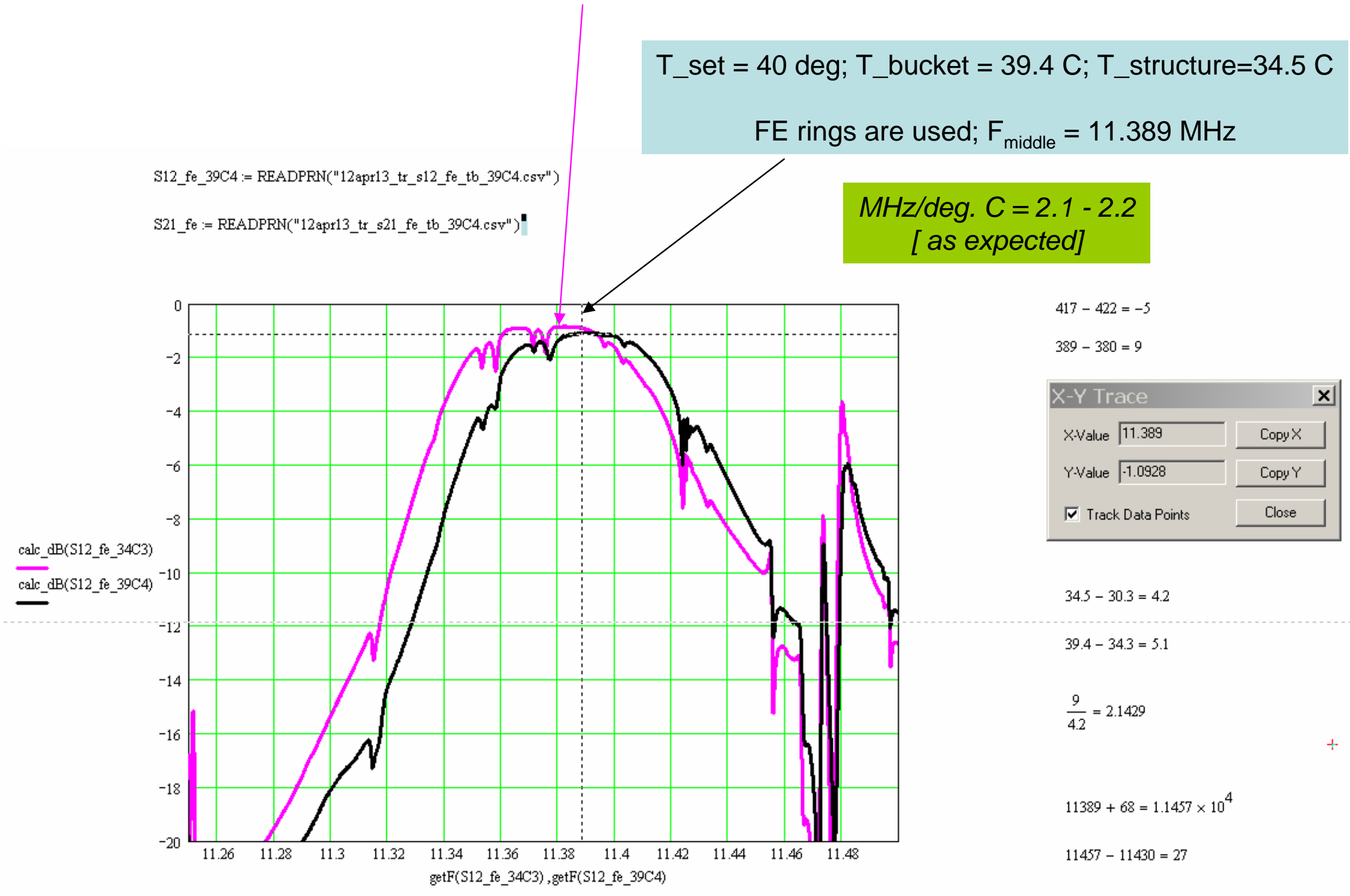


T_set $=35$ deg; T_bucket $=34.3 \mathrm{C} ; \mathrm{T} \_$structure $=30.3 \mathrm{C}$

FE rings are used; $F_{\text {middle }}=11.380 \mathrm{MHz}$

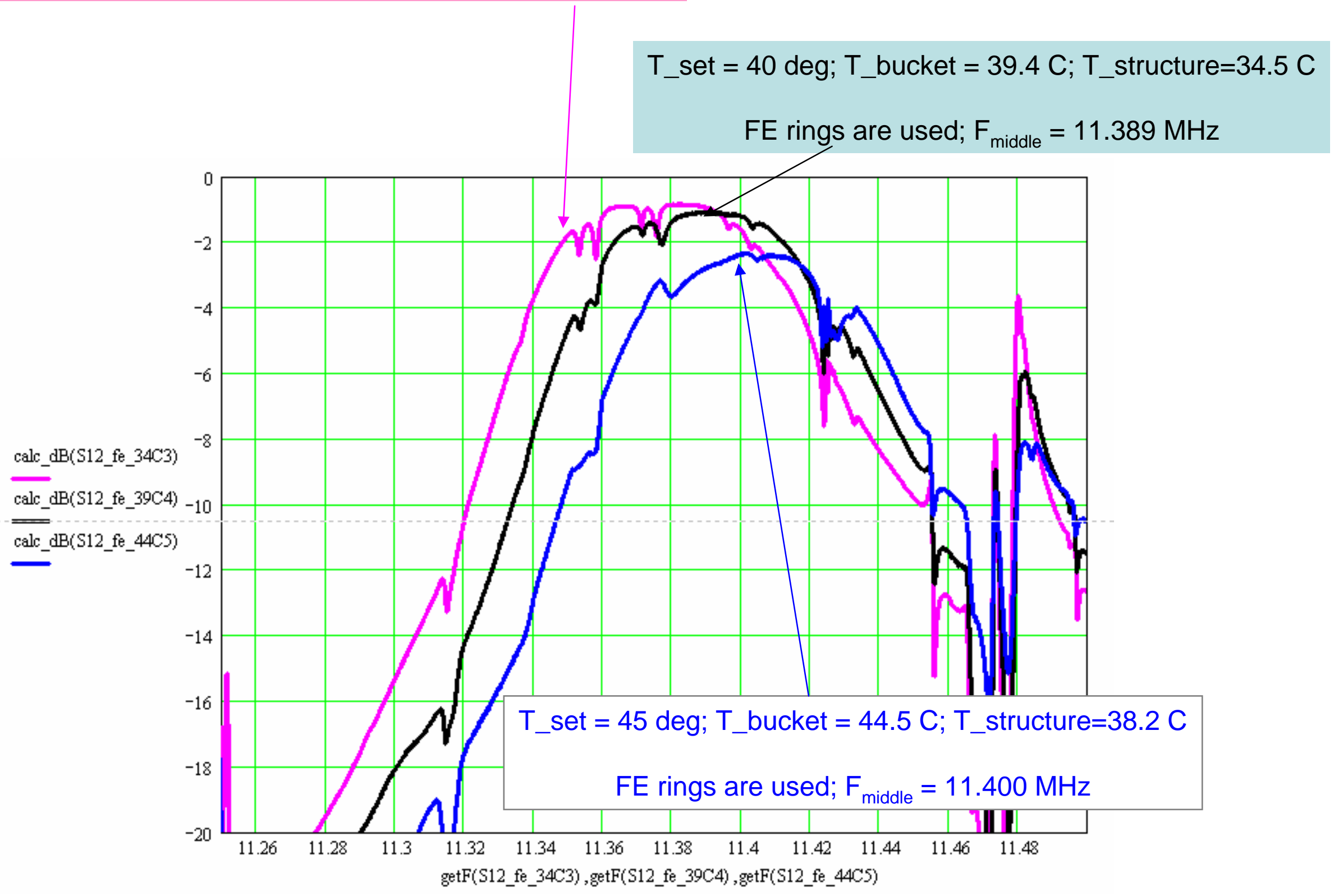


1. Should not go above $T$ _structure $=35 \mathrm{C}$

2. Perhaps chose T_structure $=32.5 \mathrm{C}$

3. Need to detune down by $-22 \mathrm{MHz}$ to operate at $\mathbf{T}$ _structure $=\mathbf{3 2 . 5} \mathbf{C}$

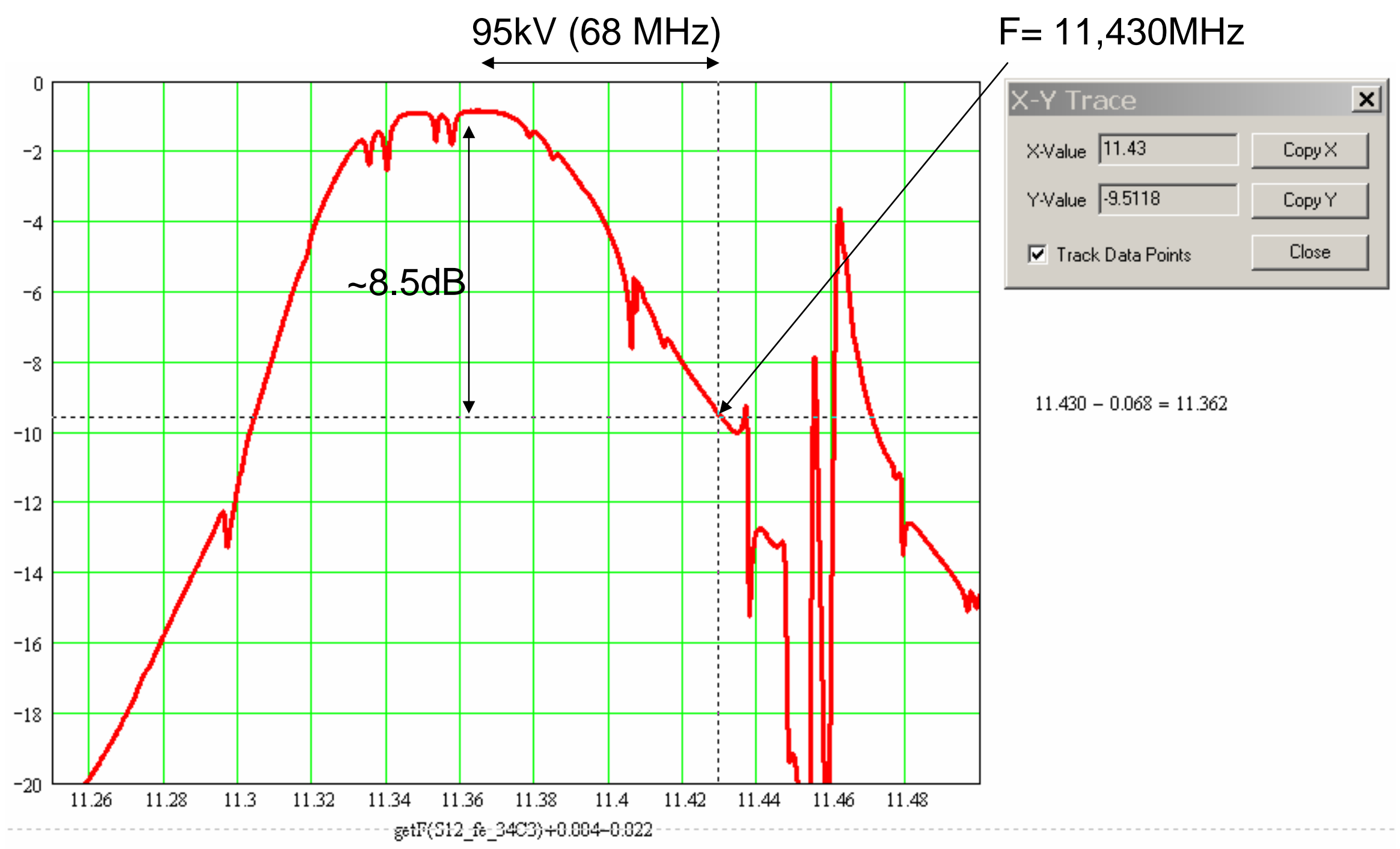


black: predicted for $\mathrm{t}=32.5 \mathrm{C}$ based on the former measurements ( $\mathrm{w} / \mathrm{FE}$ rings)

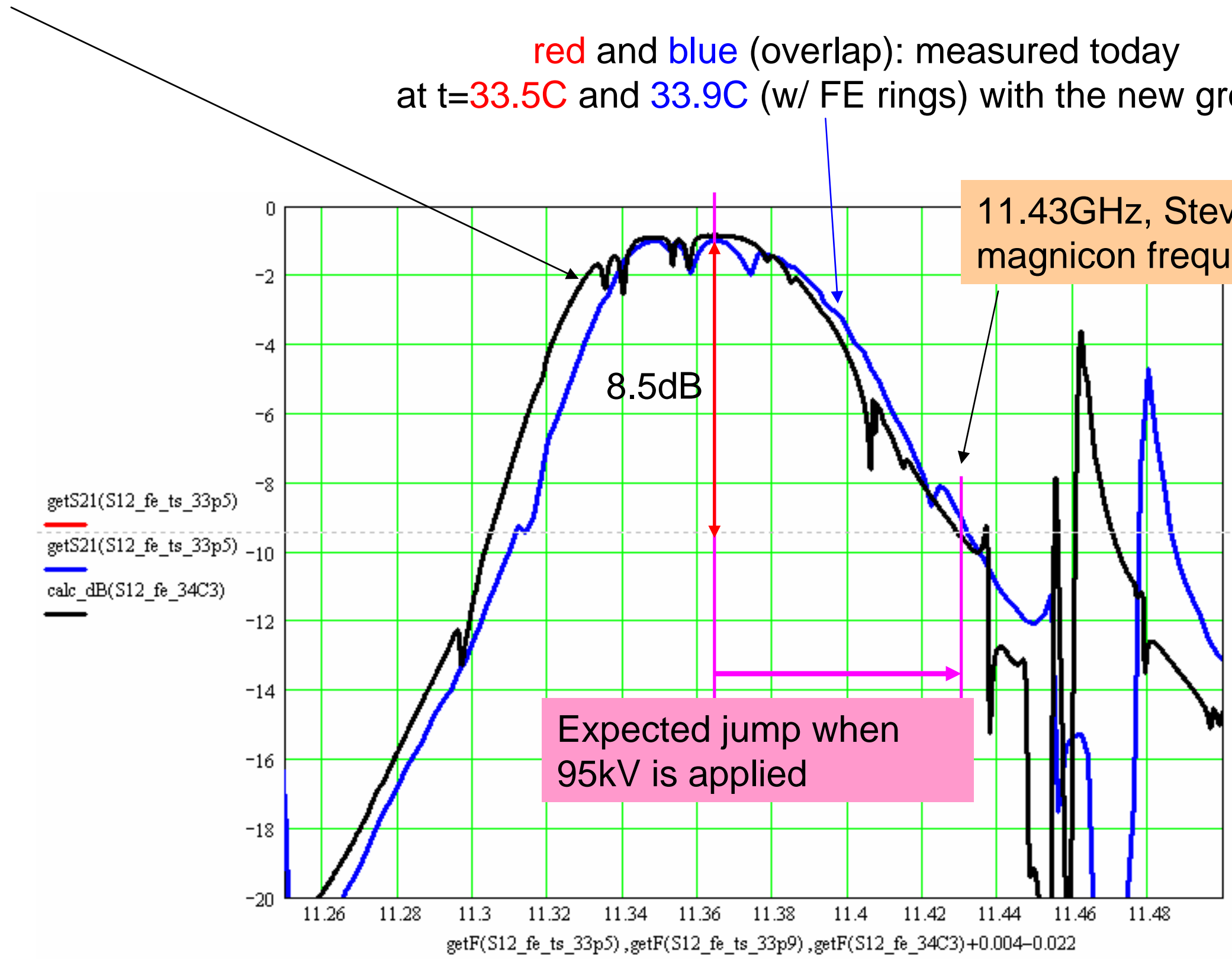




\section{Experiment schematic}
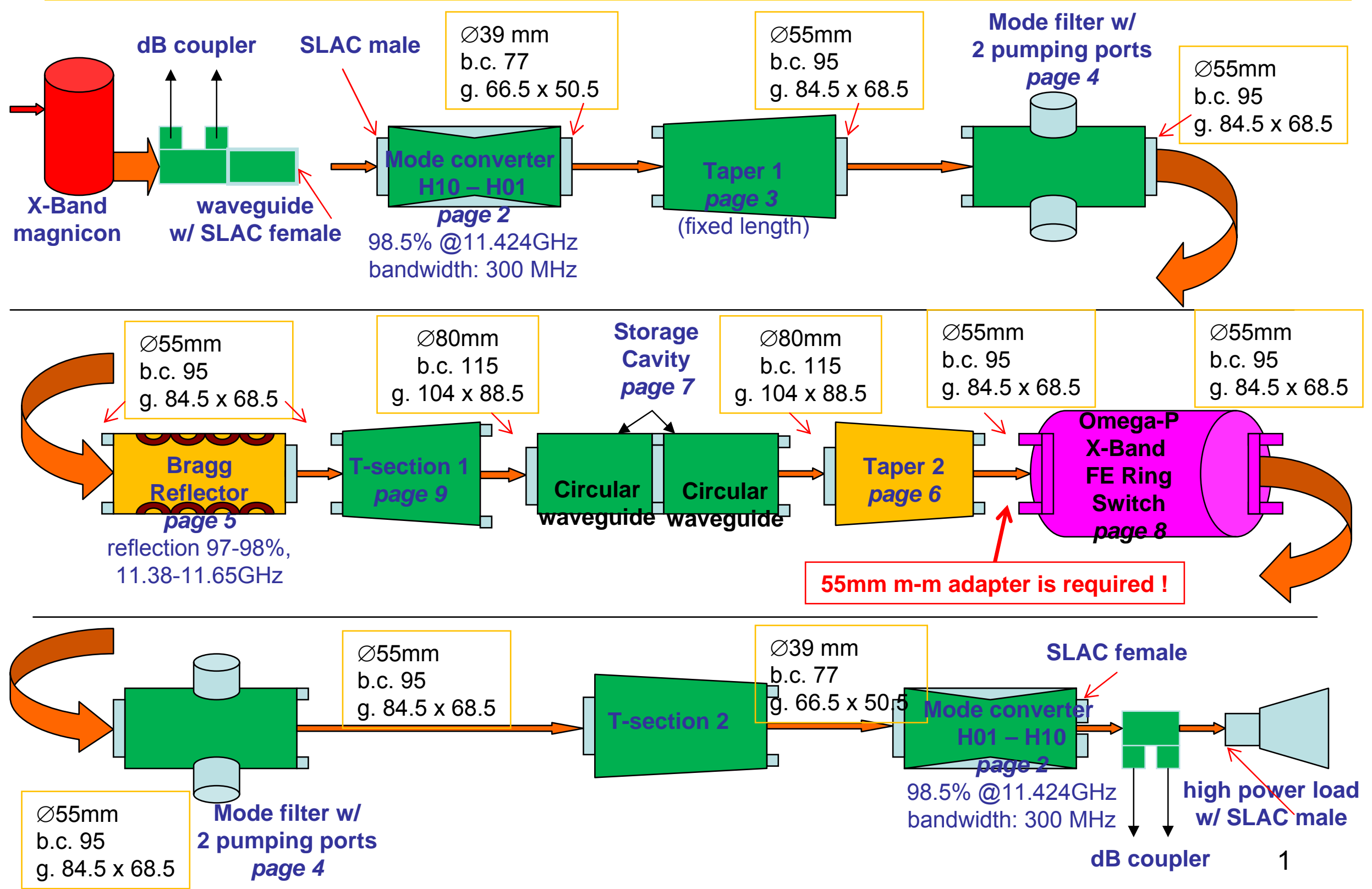


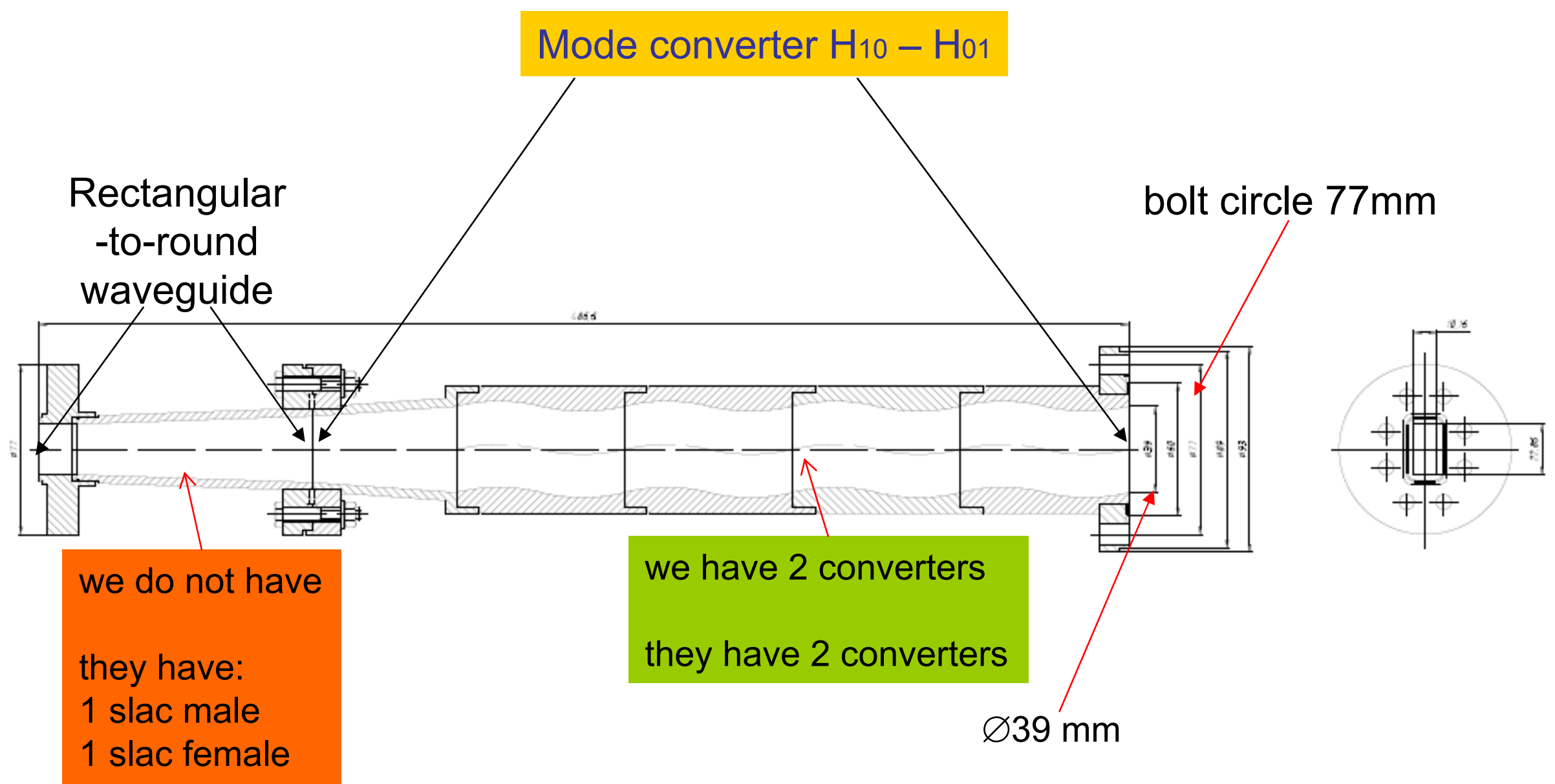


Taper 1, and Taper 4

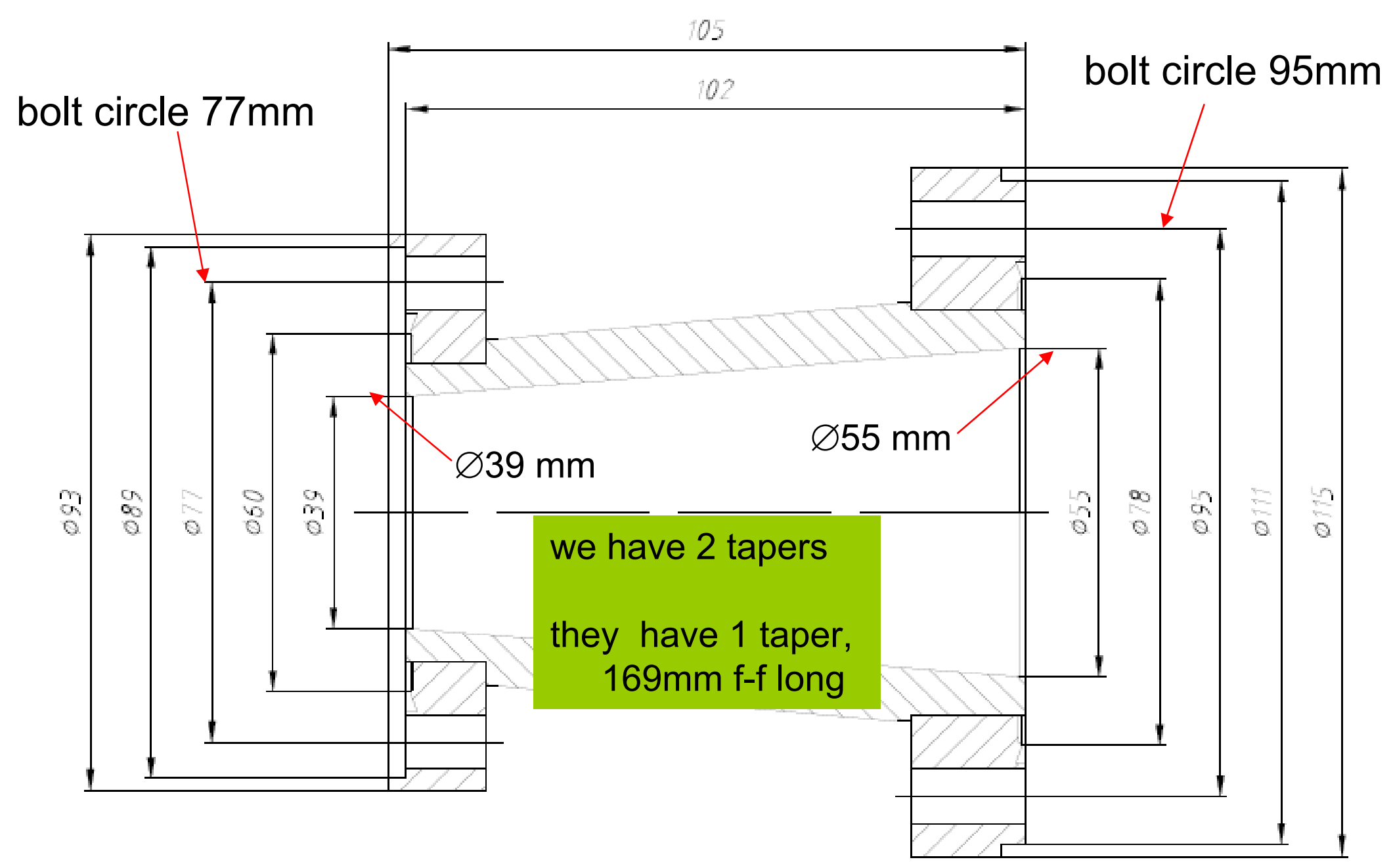


Mode filter wl

pumping port

bolt circle $95 \mathrm{~mm}$

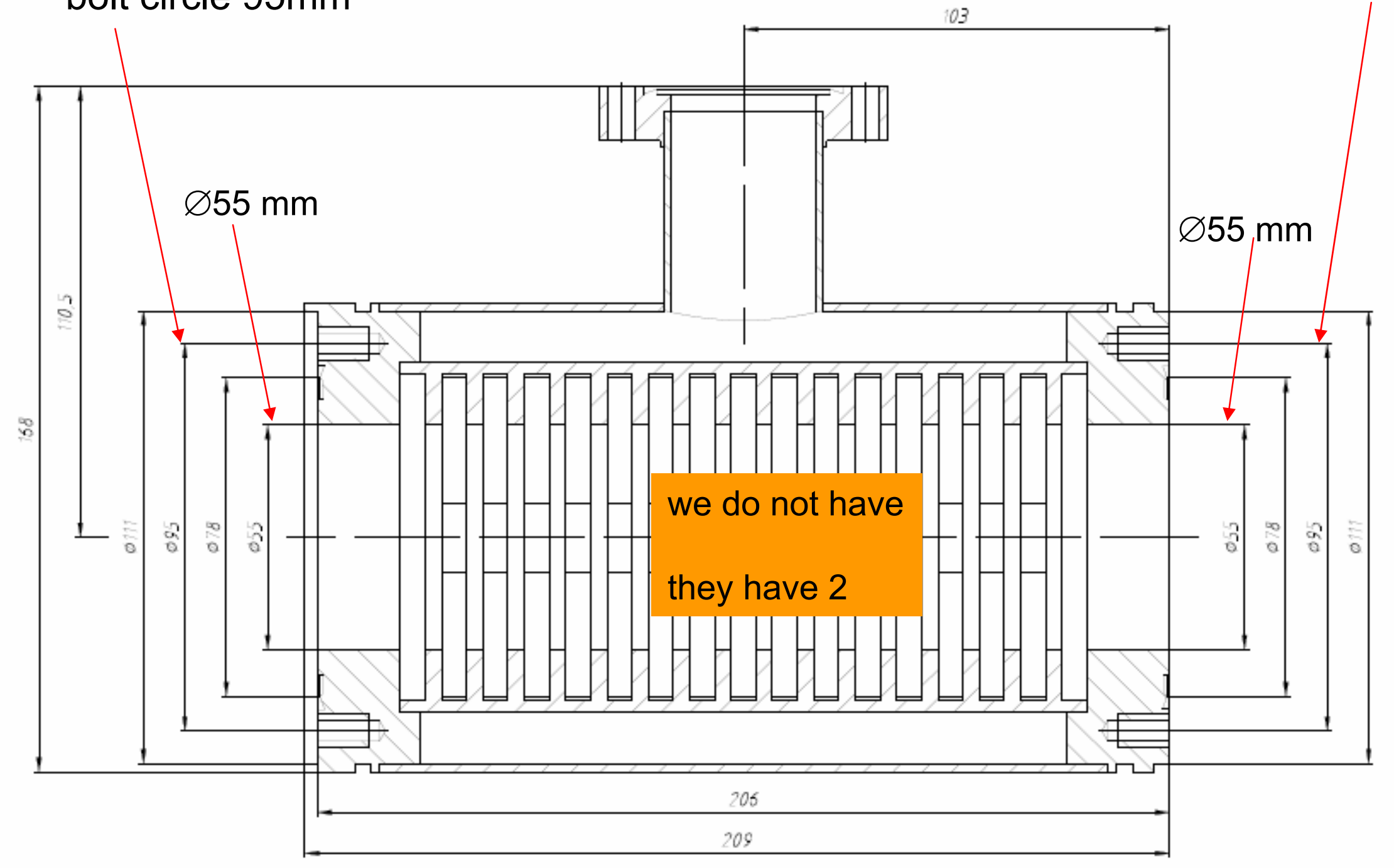




\section{Bragg \\ reflector \\ reflection $97-98 \%$, \\ $11.38-11.65 \mathrm{GHz}$}

bolt circle $95 \mathrm{~mm}$

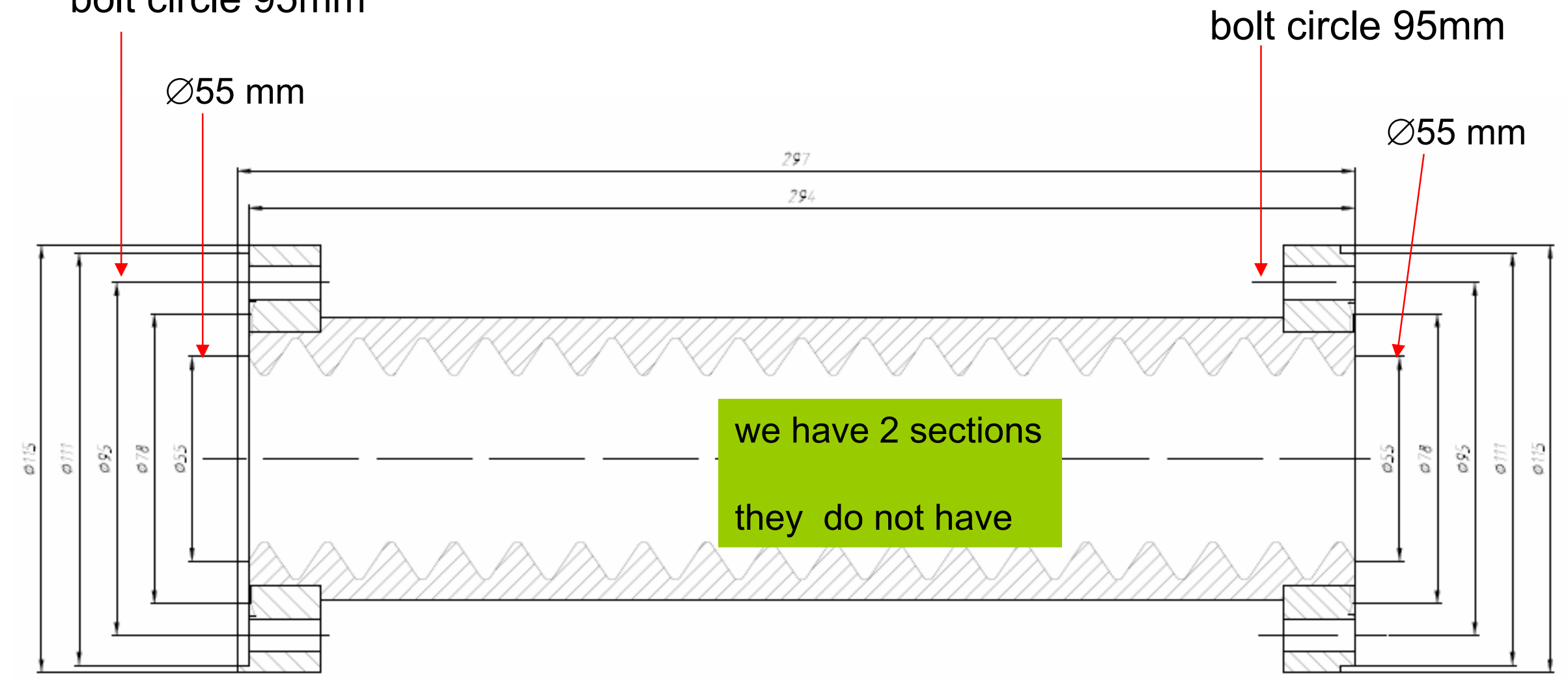


bolt circle $95 \mathrm{~mm}$

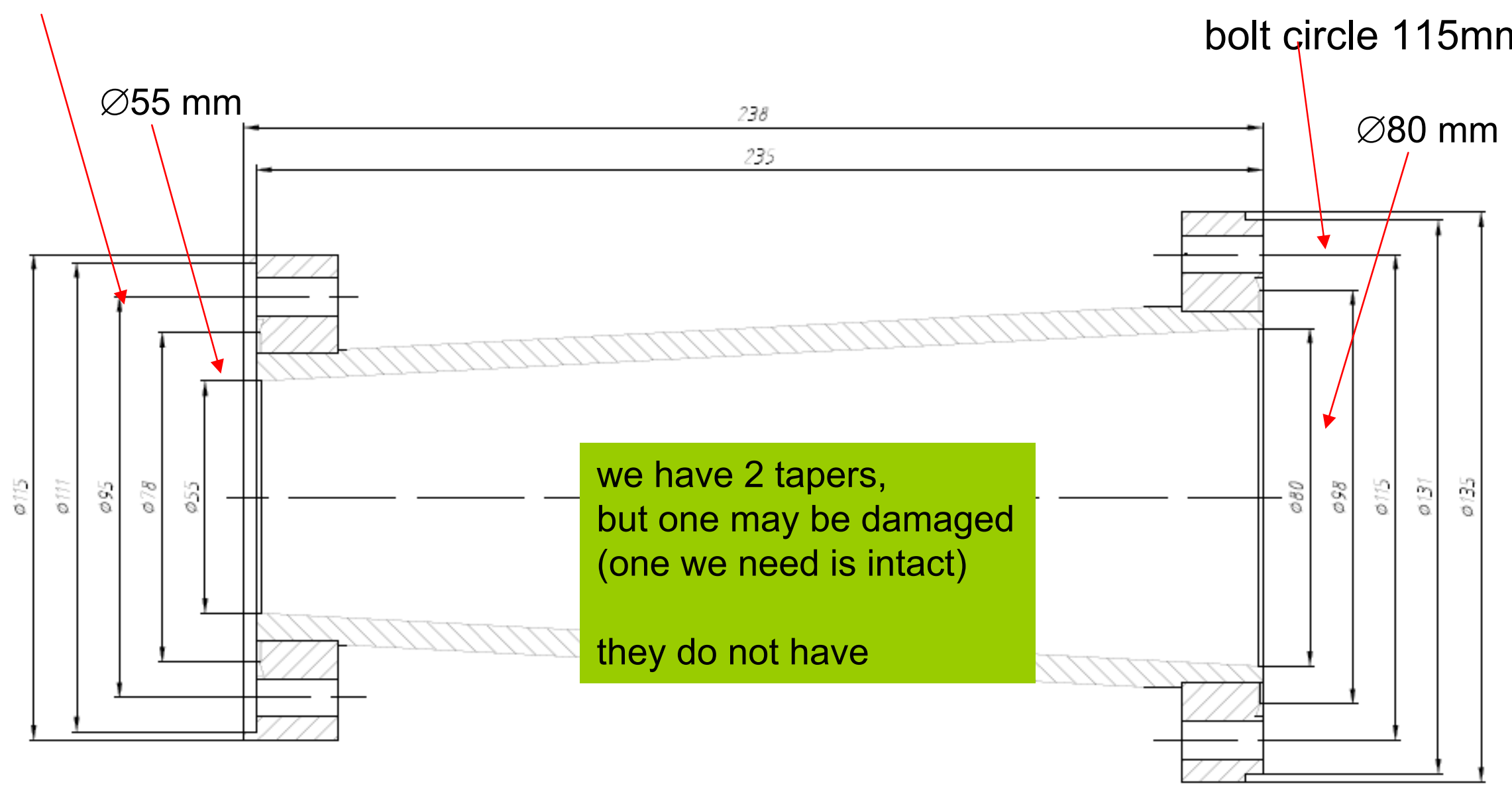


$\varnothing 80 \mathrm{~mm}$

Storage

Cavity

bolt circle $115 \mathrm{~mm}$

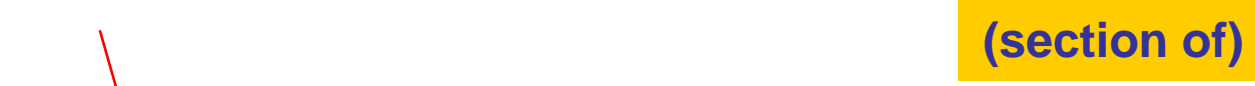

(section of)

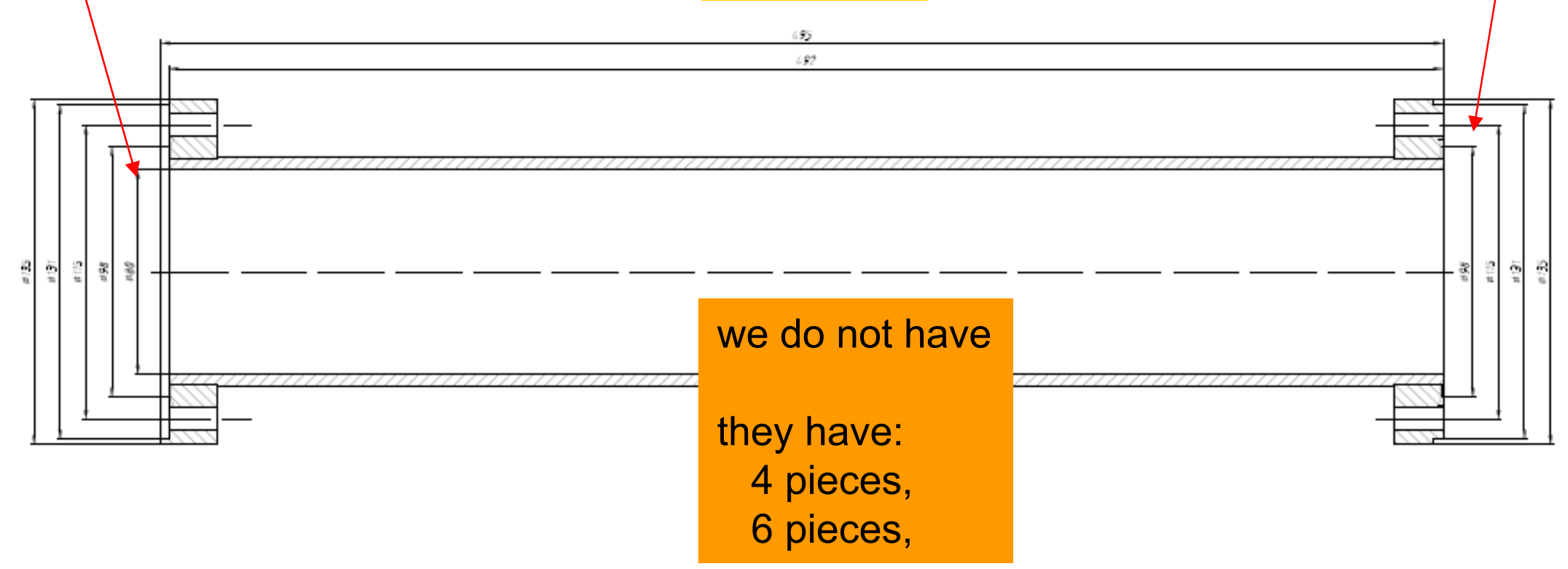




\section{Omega-P}

$X$-Band

FE Ring

Switch

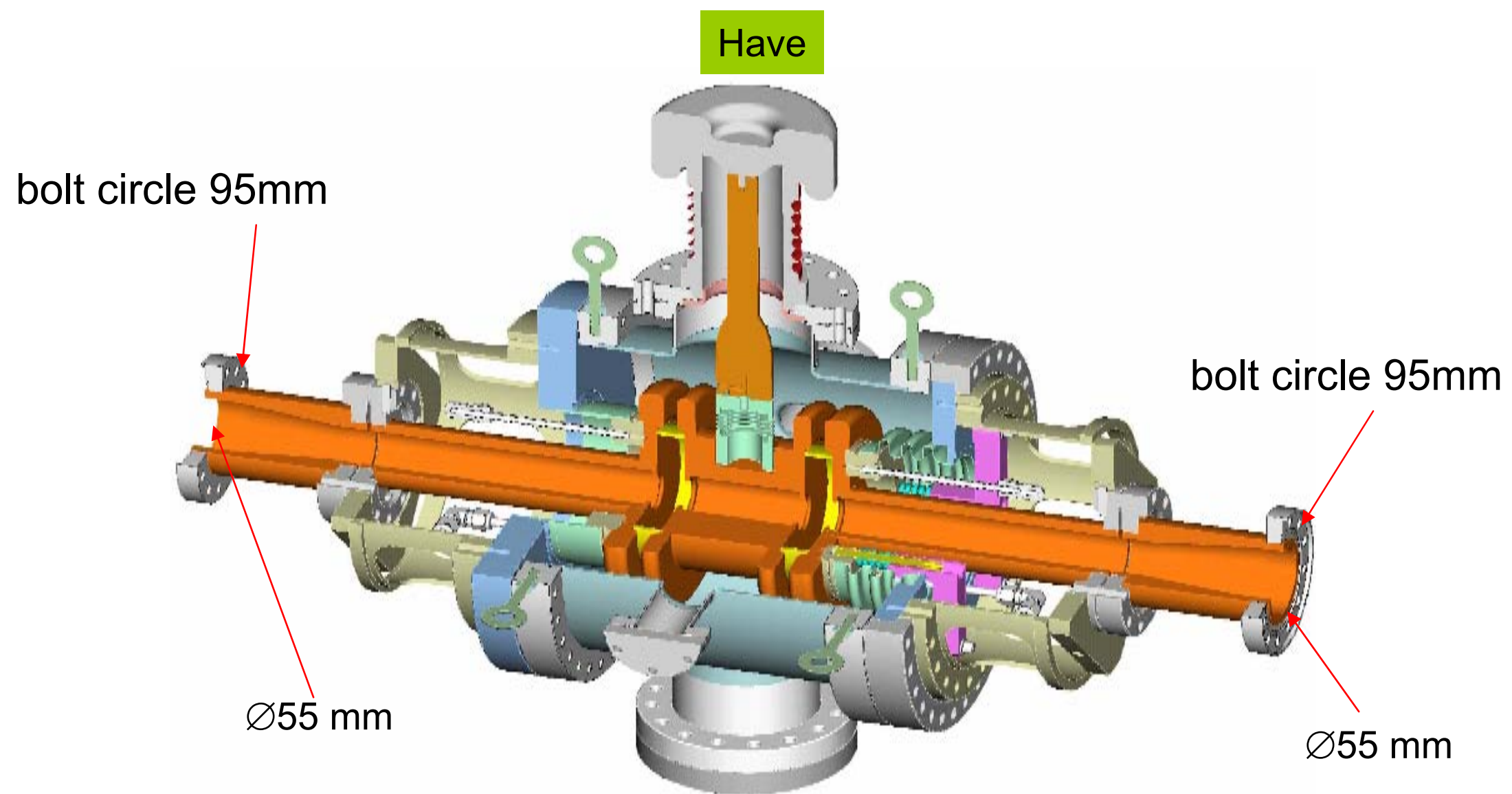




\section{Tuning section \\ (T-section)}

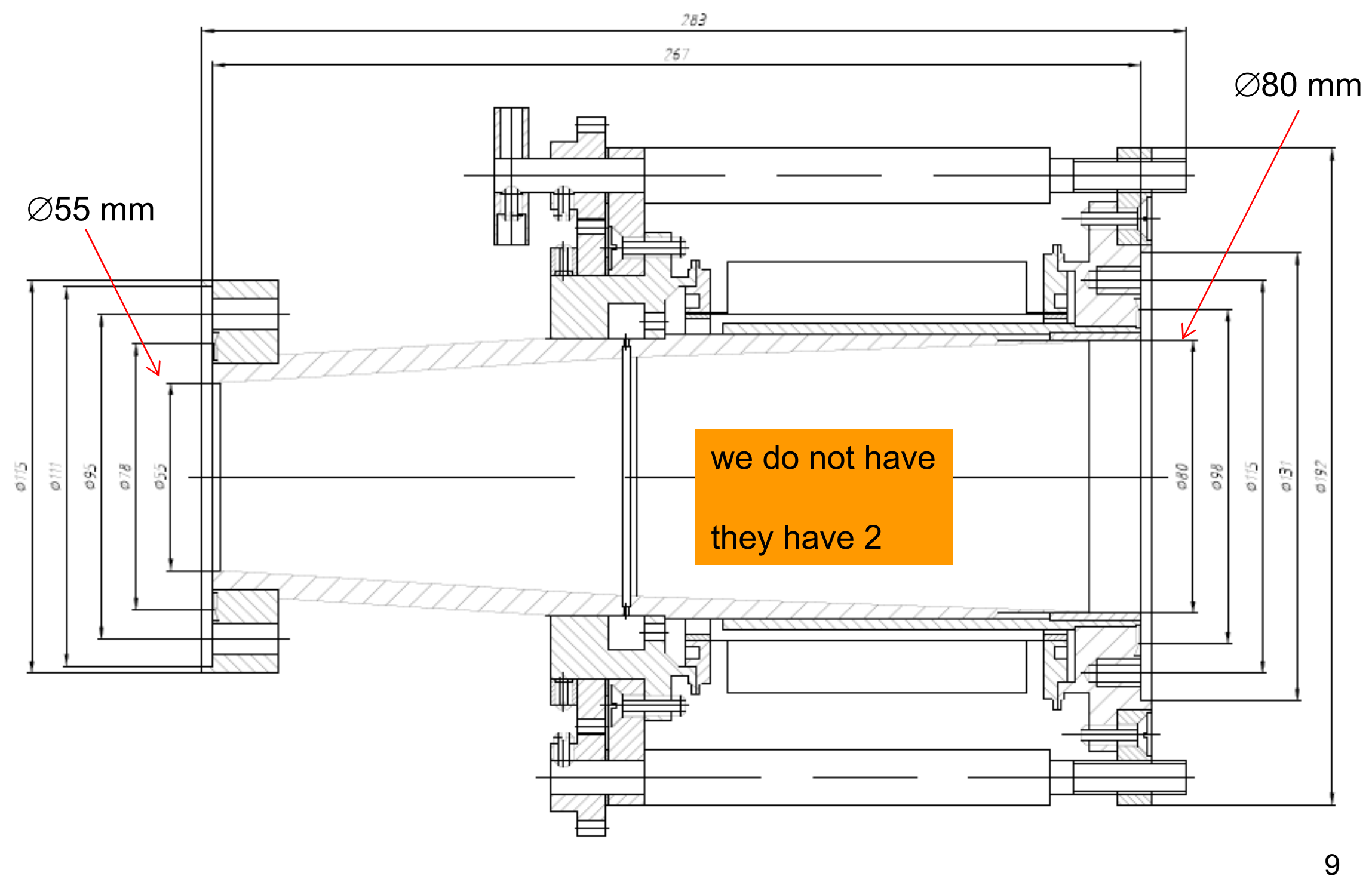




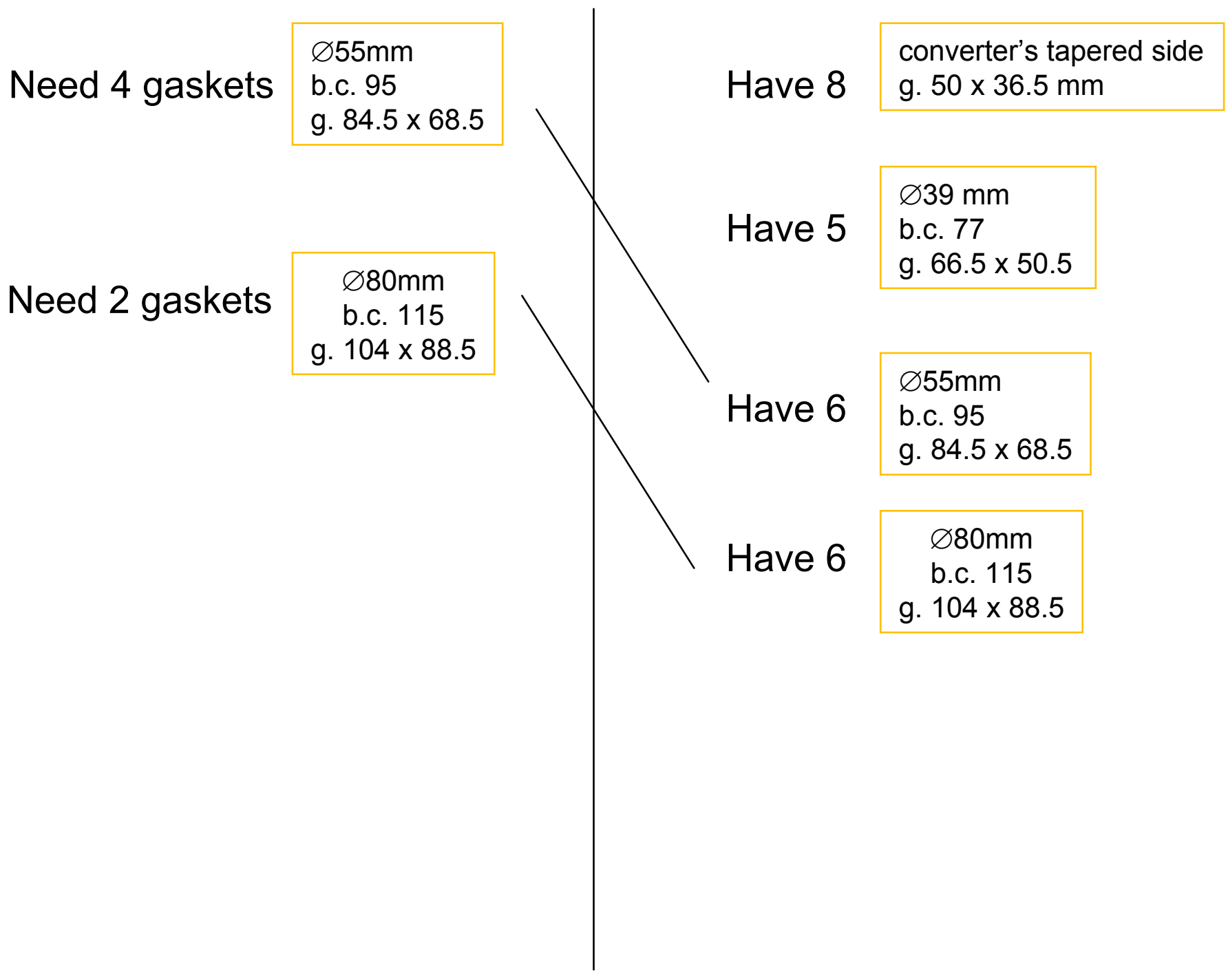




\section{Suggestion 1: schematic to find $Q_{\text {open }}, Q_{\text {close }}$, and coupling $\beta$}
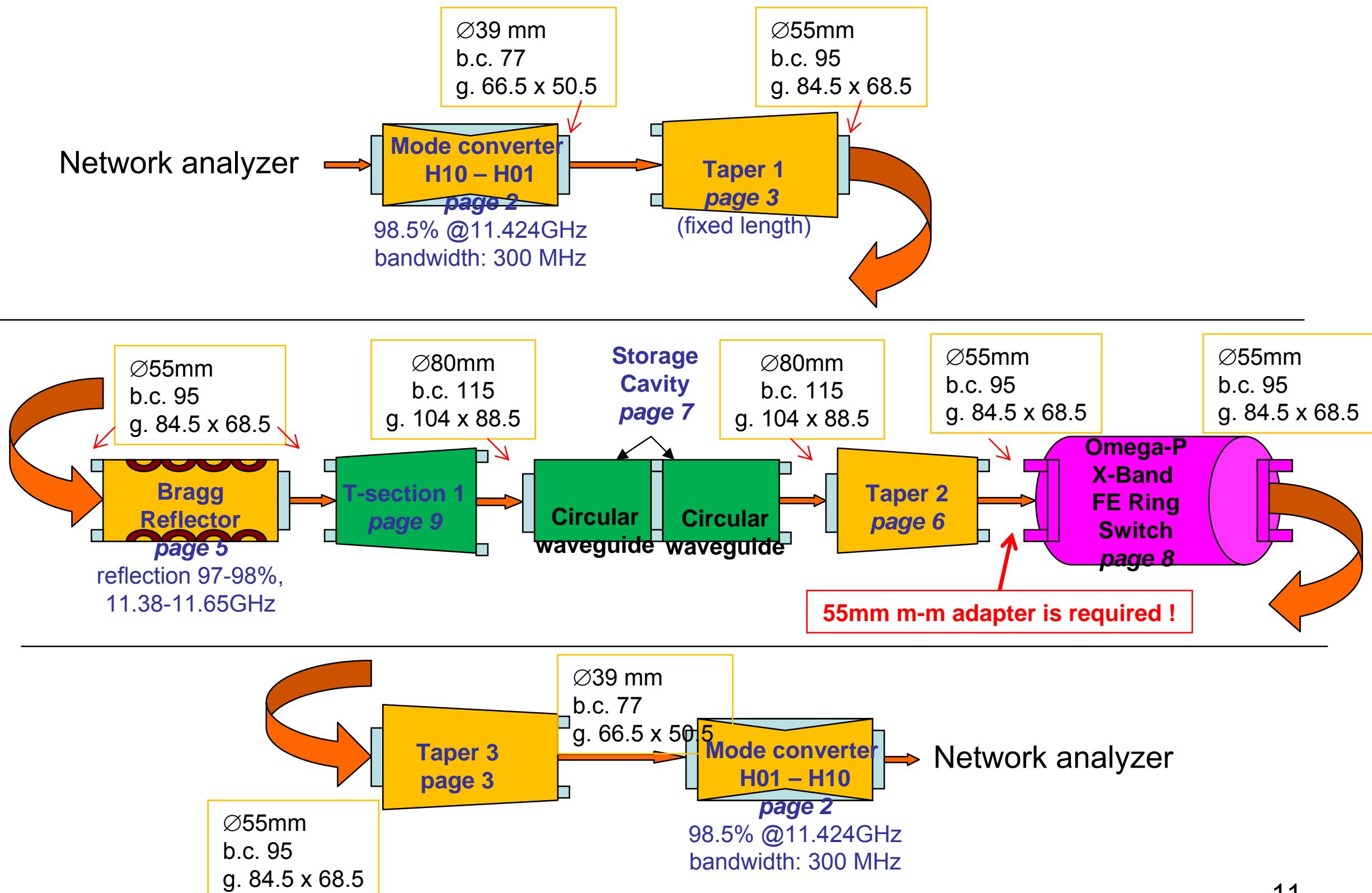
Suggestion 1: schematic to find $Q_{\text {open }}, Q_{\text {close }}$, and coupling $\beta$

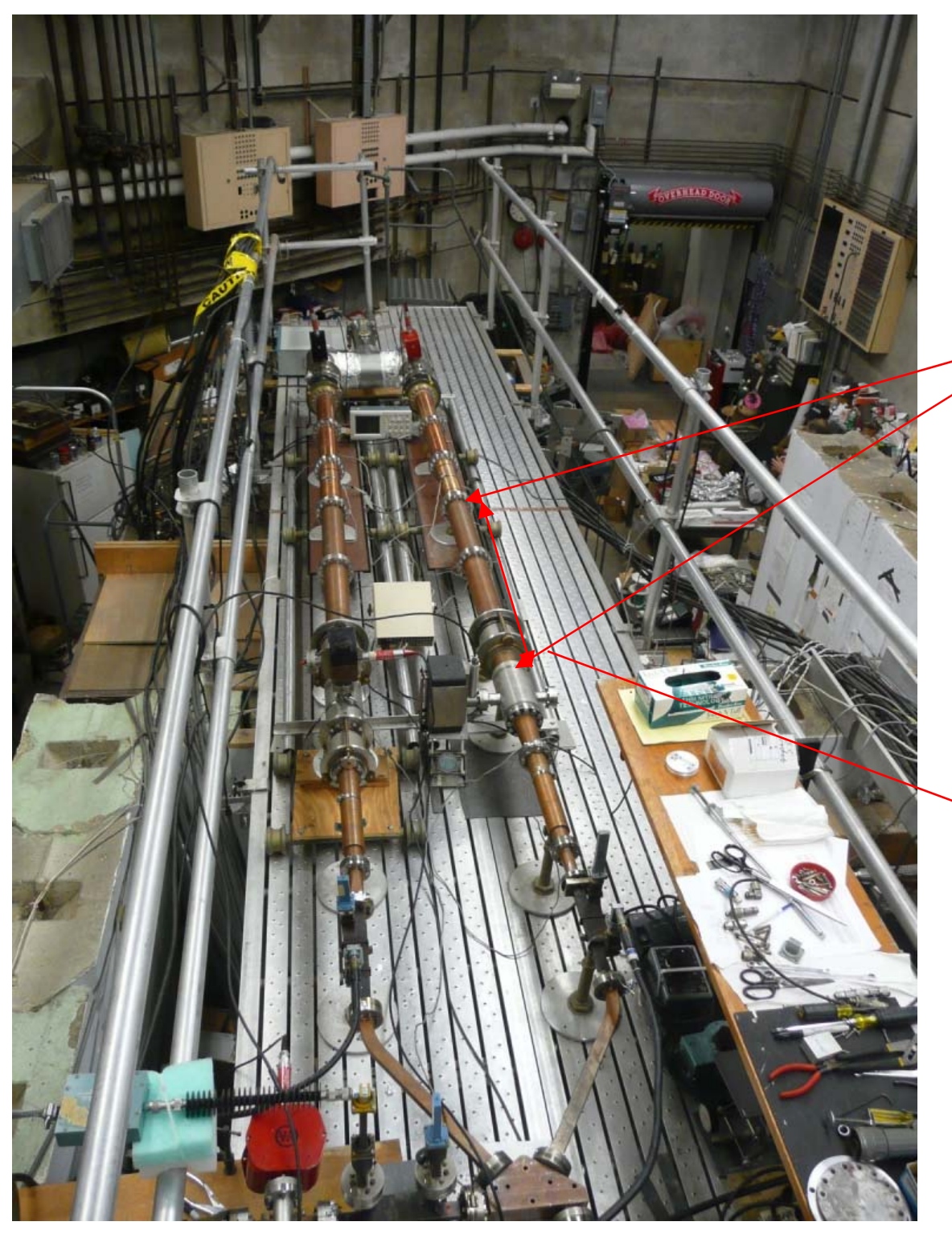

Disconnect 2 cavity sections and the T-section, and ship to Yale to use in the scheme shown on page \#11

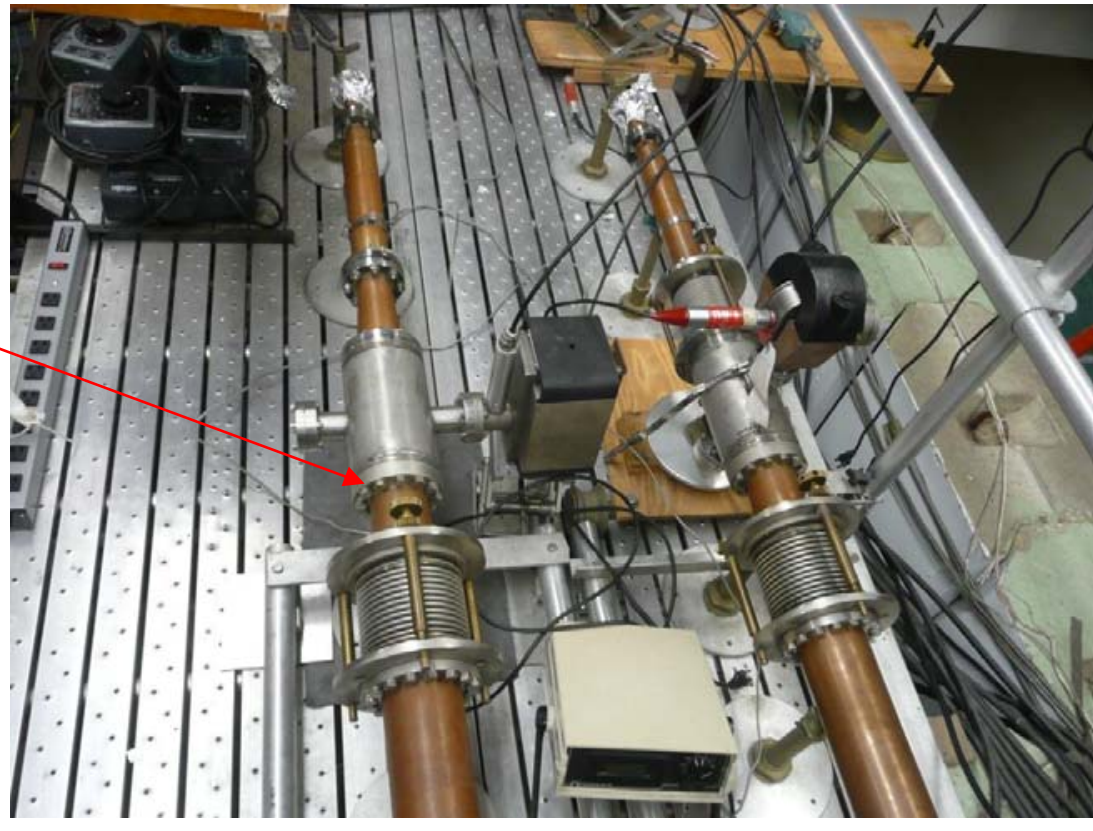


Suggestion 2: to connect the inner and outer switch volumes

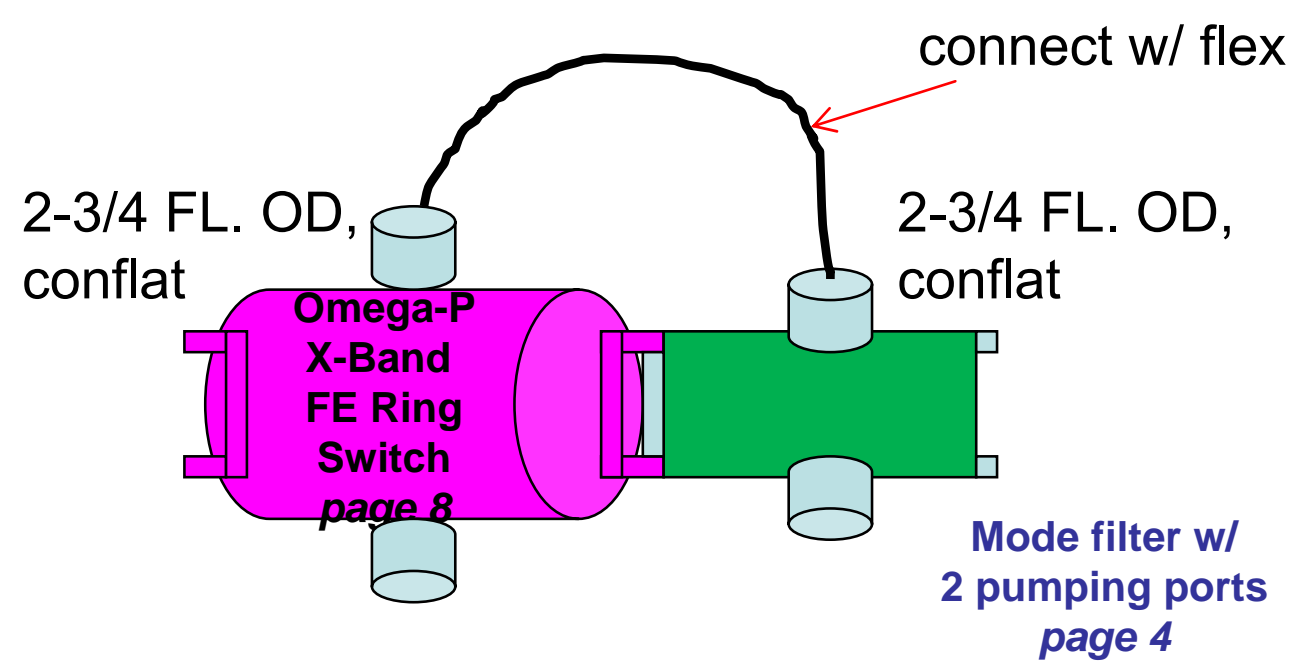




\section{RAILS}
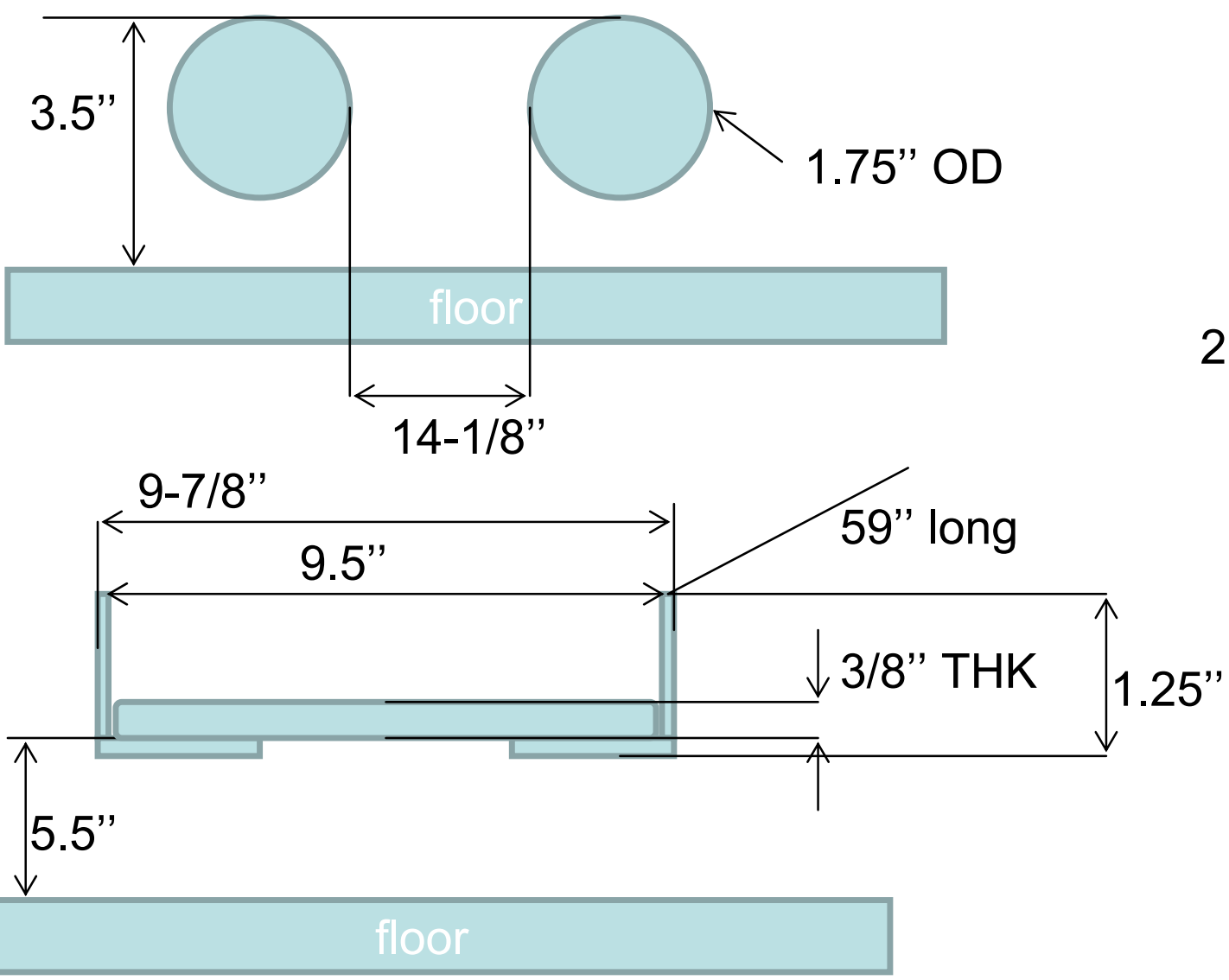

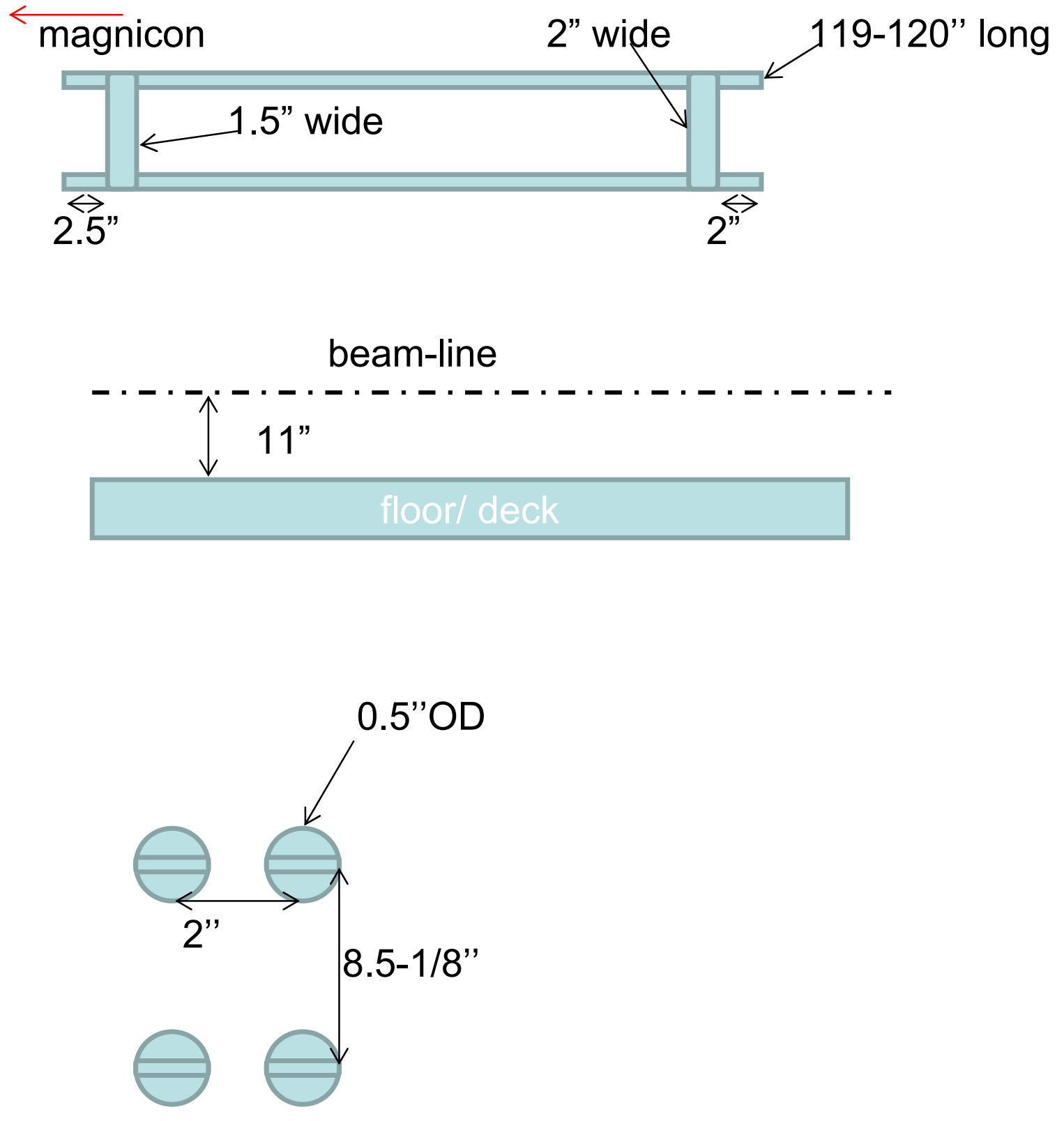


\section{Suggestion 3:}

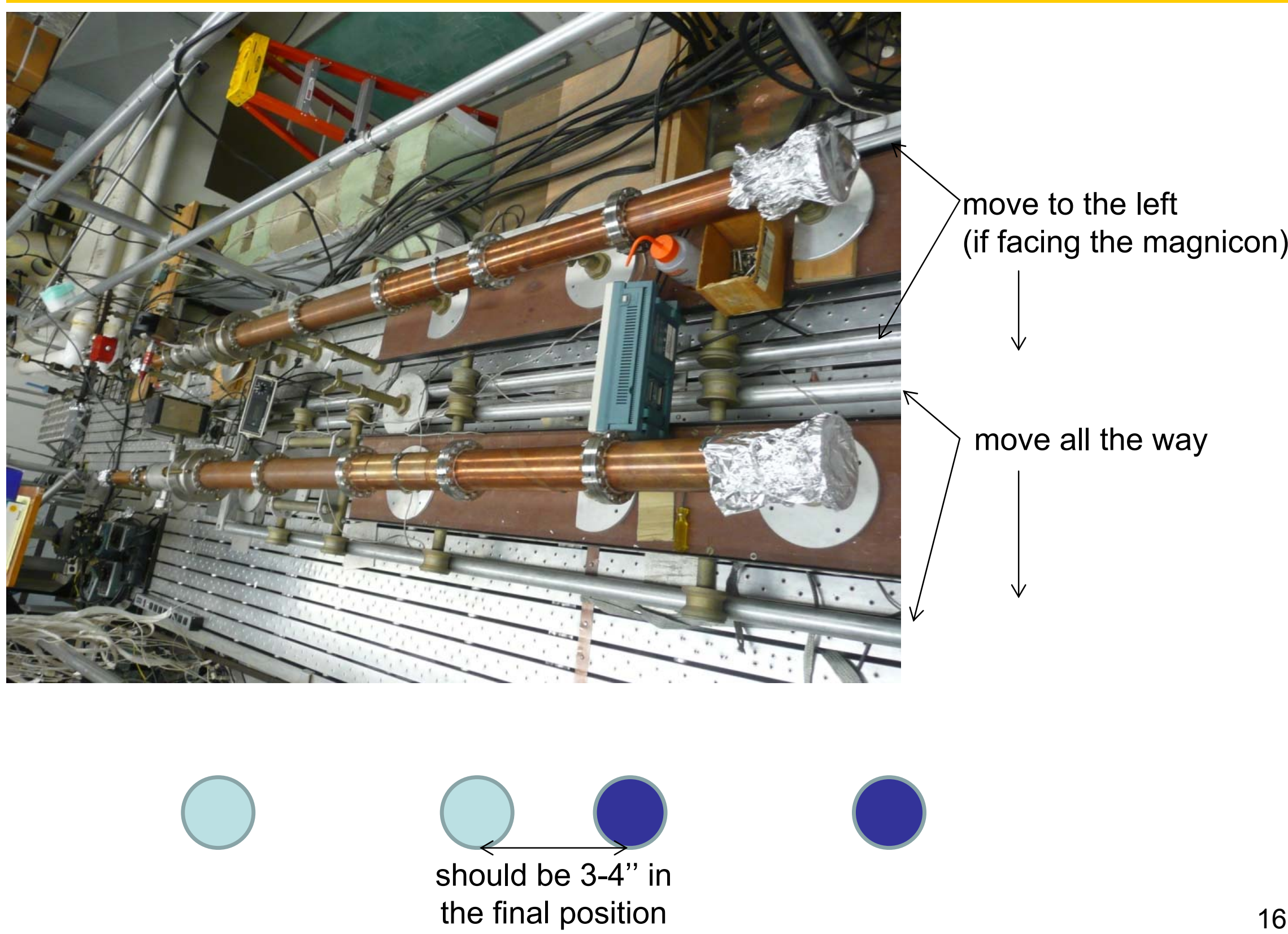




\section{Suggestion 3 (continued):}

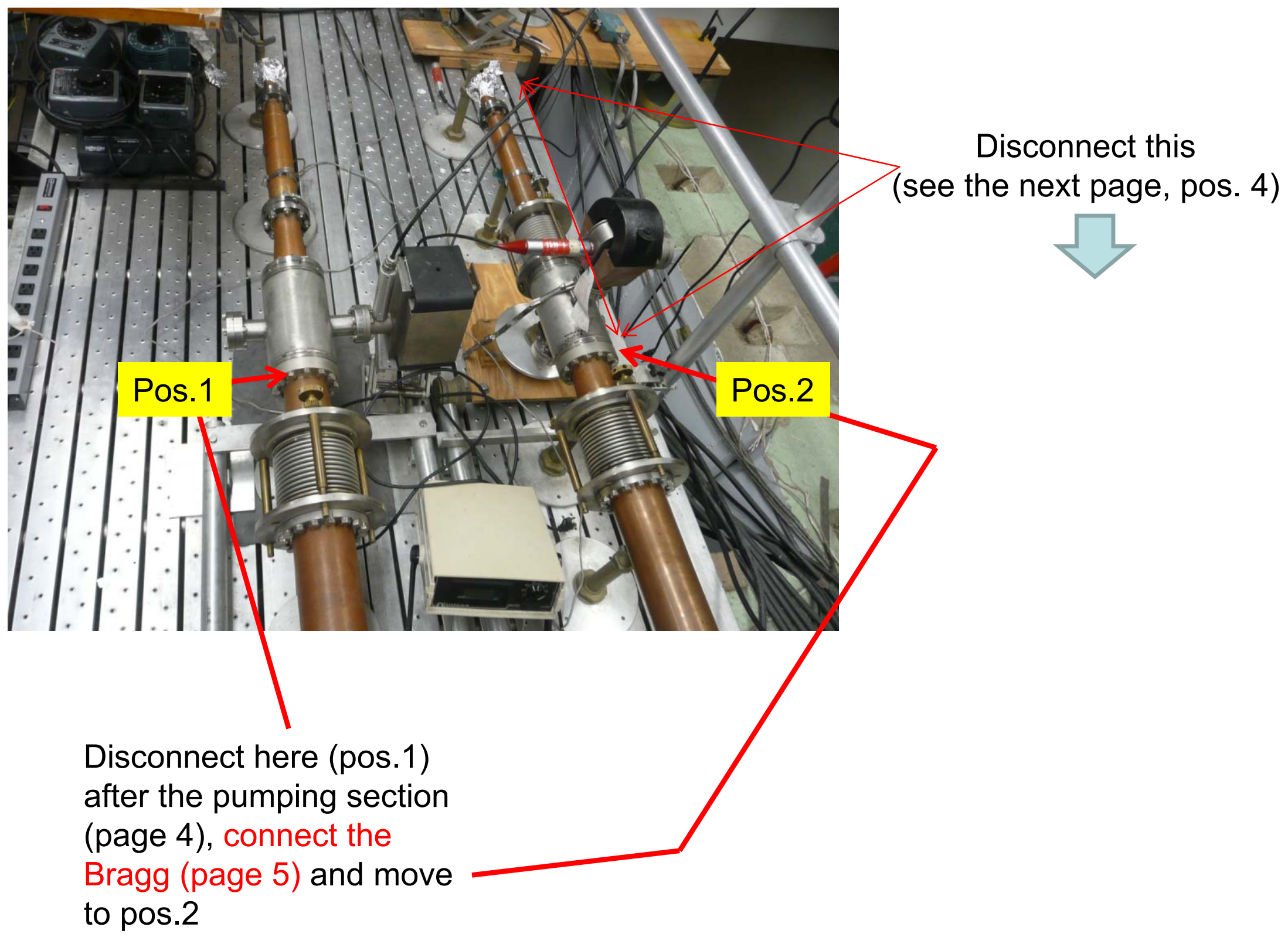




\section{Suggestion 3 (continued):}

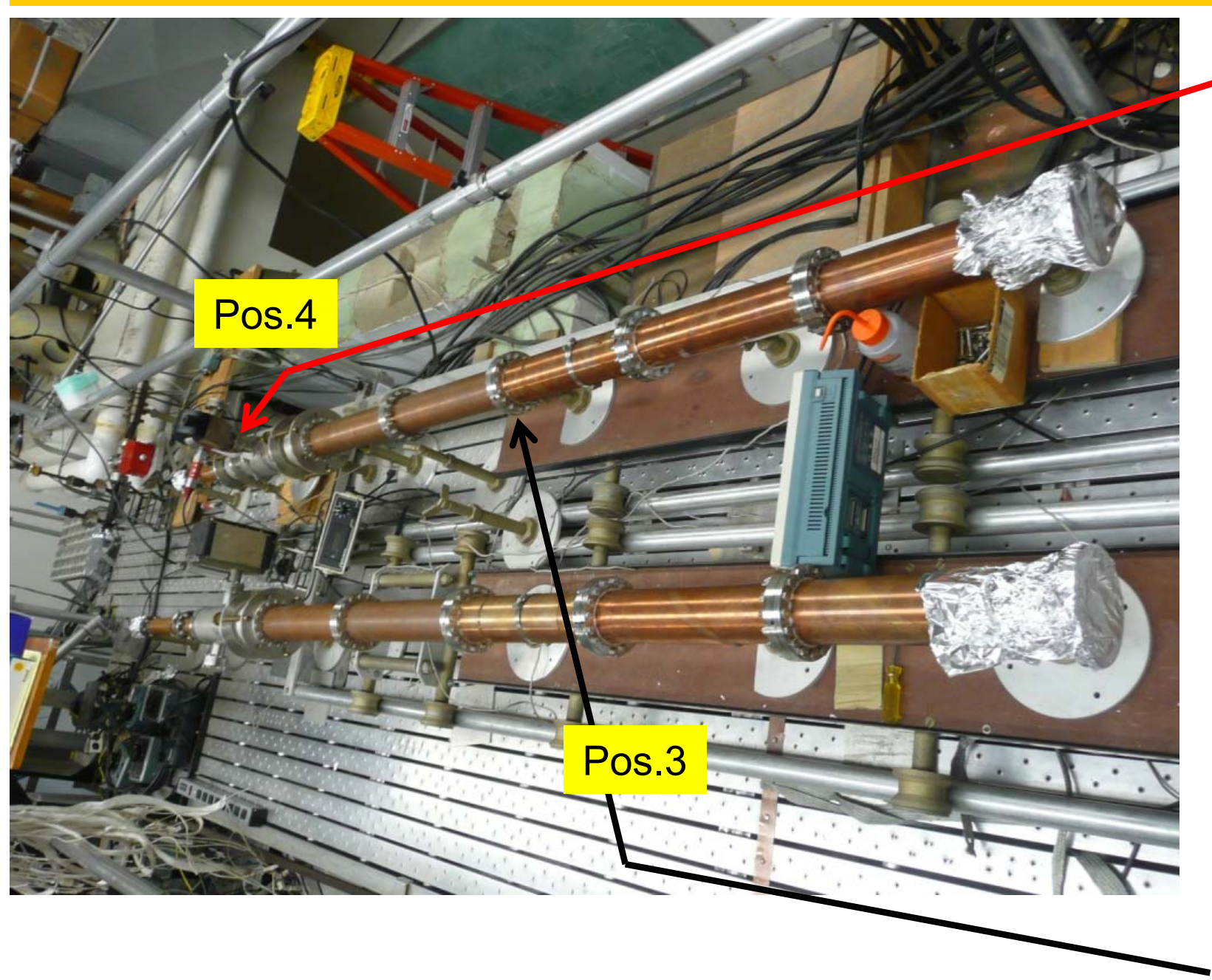

Rotate this by $180^{\circ}$ and connect to the switch

Disconnect in pos.3, and then connect to the switch via taper + adapter (see page \#1) 


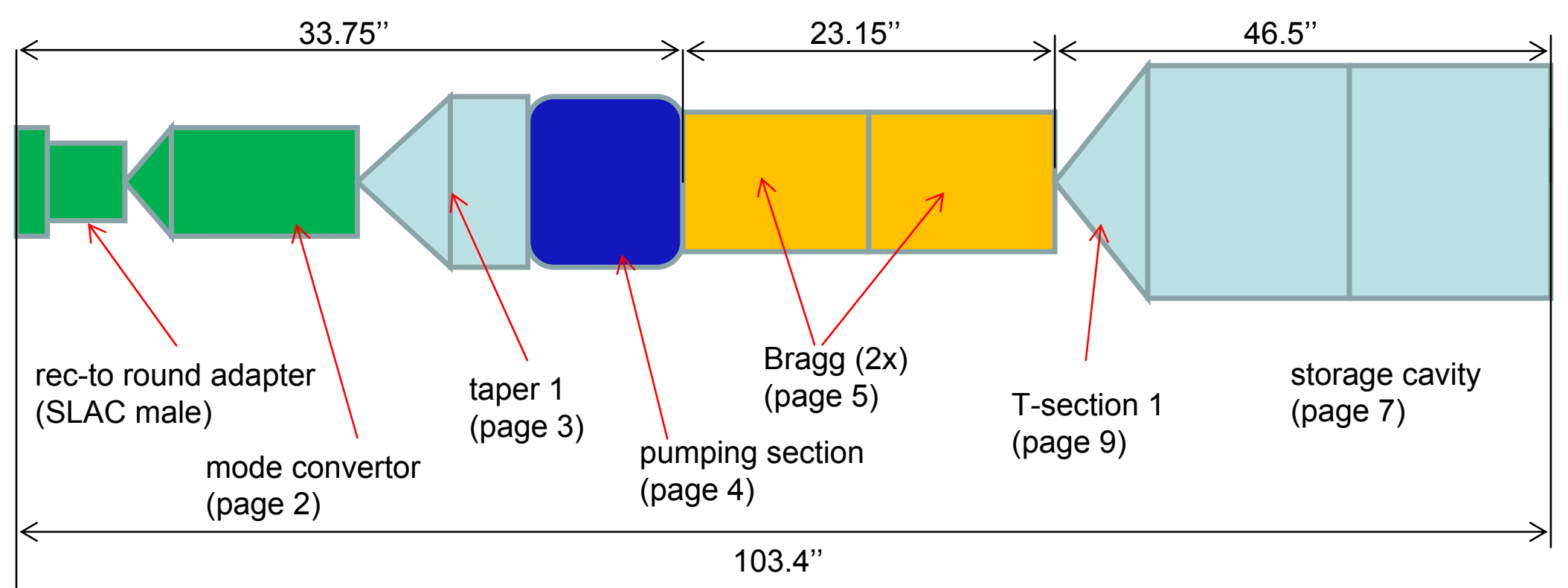

200.93"

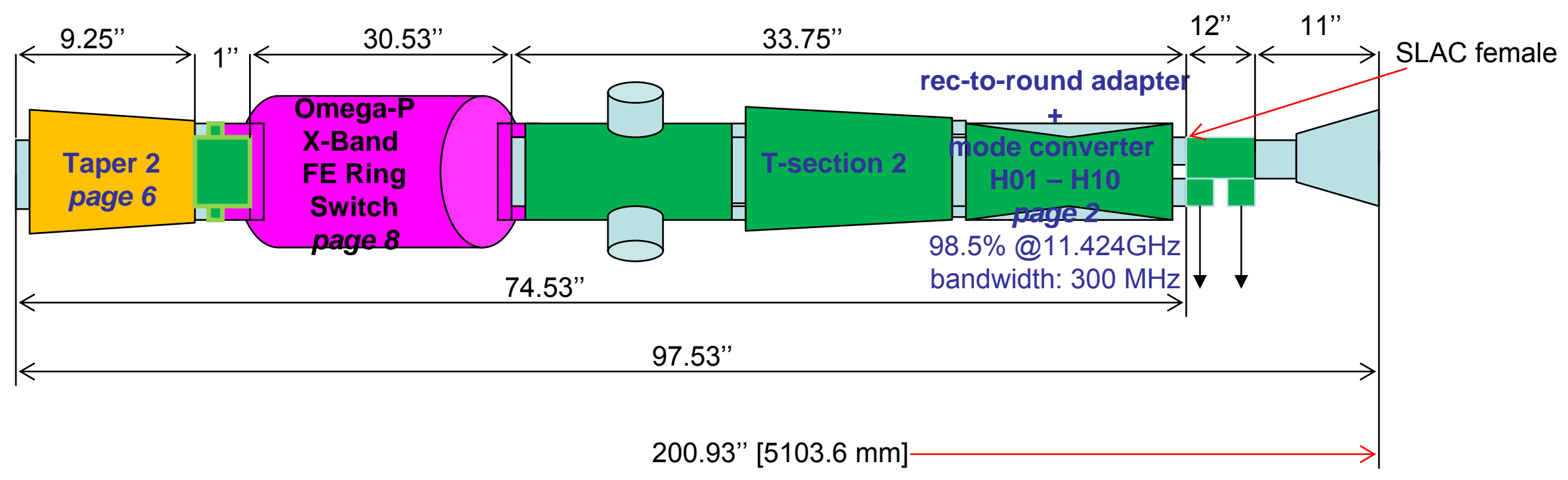

